\title{
The International Society for Biological and Environmental Repositories Presents Abstracts from Its 2020 Annual Meeting
}

\section{Roadmap to Scientific Discovery}

The abstracts that follow demonstrate the broad range of timely issues addressed in the contributed oral and poster presentations within the ISBER 2020 Educational Program Series held virtually throughout 2020 .

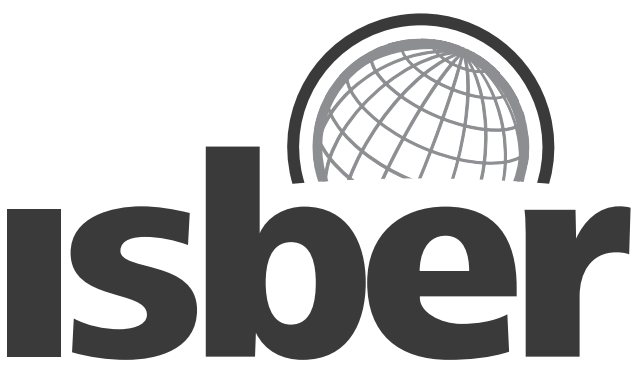

INTERNATIONAL SOCIETY FOR BIOLOGICAL AND ENVIRONMENTAL REPOSITORIES 


\section{ORAL ABSTRACTS}

\section{O-01 Freezing Tissue Biopsy Samples of Exotic Vertebrates for Future Initiation of Cell Culture}

\author{
M. L. Houck, J. A. Fronczek, C. D. Avila, A. C. Misuraca
}

San Diego Zoo Global, Escondido, California, United States

Statement of the Problem: The increasing demand for genomic quality resources (high MW DNA) highlights the importance of cell line collections. Living cells can be expanded for applications beyond what is possible with stored non-viable tissue and DNA. They can be utilized to obtain chromosome preparations, expanded to generate large quantities of highquality DNA and RNA, and can be used for somatic cell nuclear transfer and generating induced pluripotent stem cells. It is especially critical to establish and cryopreserve cell lines from species at high risk of extinction before they drop below critical population levels. However, tissue culture of exotic vertebrates requires an aseptic environment, specialized equipment, lengthy culture time, and skilled technicians that few organizations can obtain. Other challenges for acquiring quality samples from exotics include rarity of species, remote habitats, rapid tissue degradation, and permit requirements for collection and transport.

Proposed Solution: A protocol developed at San Diego Zoo's Frozen Zoo ${ }^{\circledR}$ (the largest viable vertebrate biobank) provides a way to preserve samples with minimal training and equipment. Tissue biopsy samples are quickly processed with dimethyl sulfoxide (DMSO) and stored indefinitely in liquid nitrogen to halt sample degradation until transport to a qualified cell culture lab can be arranged. The method has been successful for remote collection sites and for post-mortem animals as long as viable cells remain in the sample. When multiple sampling events are desired over several days and where refrigeration opportunities are limited, the reagents are aliquoted into single-use volumes prior to collection procedures. This minimizes the potential for contamination that can occur during multiple sampling from a single container.

Conclusions: Freezing tissue samples using a method appropriate for future initiation of cell culture requires less time and fewer resources than tissue culture and provides a way to capture a source of genetic variation that would otherwise be lost.

\section{O-02 Establishment of the Prognostic Index of Lung Squamous Cell Carcinoma Based on Immunogenomic Landscape Analysis}

J. Ren ${ }^{1}$, J. Zhang ${ }^{2}$, J. Zhang ${ }^{3}$, G. Wang ${ }^{1}$, H. Shi ${ }^{1}$, Y. Fang ${ }^{1}$, M. Yu ${ }^{1}$, N. Zhang ${ }^{2}$, C. Xie ${ }^{2}$, Y. Gong

${ }^{1}$ Department of Biological Repositories, Zhongnan Hospital of Wuhan University, Wuhan, China, ${ }^{2}$ Department of Radiation and Medical Oncology, Zhongnan Hospital of Wuhan University, Wuhan, China, ${ }^{3}$ Department of Occupational and Environmental Health, Qingdao University, Qingdao, China
Background: The incidence of lung squamous cell carcinoma (LUSC) increased substantially in recent years. Systematical investigation of the immunogenomic pattern is critical to improve the prognosis of LUSC.

Methods: Based on the TCGA dataset, we integrated the immune-related genes (IRGs) expression profile and the overall survival of 502 patients with LUSC. The survival-related and differentially-expressed IRGs in LUSC patients were evaluated by univariate Cox regression and lasso regression analysis. With the assist of computational biology, we also investigated the latent properties and molecular mechanisms of these LUSC-specific IRGs. By applying multivariate Cox analysis, a new prognostic indicator based on IRGs was established. We also used CIBERSORT algorithms to analyze immune infiltration of LUSC. Both gene set enrichment analysis (GSEA) and principal component analysis (PCA) was carried out for functional annotation.

Results: Twenty-three differentially expressed IRGs were notably associated with the outcomes of LUSC patients. Kyoto Encyclopedia of Genes and Genomes analysis showed that these IRGs were involved in the Toll-like receptor, cyclic adenosine monophosphate, tumor necrosis factor, and interleukin-17 signaling pathways. We also investigated the relationship between these genes and transcription factors, and mapped the regulatory network by Cytoscape. By applying multivariate Cox analysis, a new prognostic model based on 13 IRGs, including RNASE7, EDN2, LTB4R2, GCGR, TRAV39, MMP12, PLAU, SEMA4C, JUND, AMH, JAG1, FGFR4, and CXCL5, was established. This model performed well in the prognostic forecast, and was also related to the infiltration of immune cells. Besides, the high-risk groups and the low-risk groups exhibited distinct layout modes in PCA analysis, and GSEA results showed that different immune status among these groups.

Conclusions: In summary, our research screened out clinically significant IRGs and proved the significance of IRGbased, individualized immune-related biomarkers in monitoring, prognosis, and discern of LUSC.

\section{O-03 Biochemically Tracked Variability of Blood Plasma Thawed-State Exposure Times in a Multisite Collection Study}

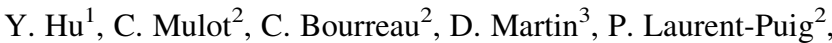
L. Radoi ${ }^{3,4}$, P. Guenel ${ }^{3}$, C. R. Borges ${ }^{1}$

${ }^{1}$ School of Molecular Sciences \& The Biodesign Institute, Arizona State University, Tempe, Arizona, United States, ${ }^{2}$ CRB EPIGENETEC, INSERM, Paris, France, ${ }^{3}$ CESP, INSERM, Paris, France, ${ }^{4}$ Faculty of Dental Surgery, University Paris Descartes, Paris, France

Background: Multisite collection study guidelines generally prescribe uniform collection and initial processing conditions for plasma and serum (P/S) samples. But given personnel, infrastructure, and unforeseen circumstantial variability between 
sites, this is often imperfectly achieved. Moreover, prior to final analysis, freeze-thaw histories are often poorly documentedparticularly at the individual aliquot level. In turn, the heterogeneity that results between specimens creates the potential for confounding or even misleading research results. Here we evaluated the effect of initial pre-storage delay and initial storage temperature on P/S sample integrity by applying a novel thawed-state exposure lab test called $\Delta \mathrm{S}$-Cys-Albumin to a set of 413 plasma samples collected under a single clinical study but at 12 different sites.

Methods: We recently reported a $10-\mu \mathrm{L}$ dilute-and-shoot, intact-protein LC/MS assay called $\Delta \mathrm{S}$-Cys-Albumin that quantifies cumulative exposure of $\mathrm{P} / \mathrm{S}$ to thawed conditions based on the change in relative abundance of the oxidized (S-cysteinylated) proteoform of albumin (S-Cys-Albumin) in the native sample to that of an aliquot of the sample intentionally driven to its maximum oxidation state. Low $\Delta \mathrm{S}-\mathrm{Cys}$-Albumin values correspond to poorly handled samples.

Results: Major differences $(\mathrm{p}<0.001)$ were observed between groups of plasma samples with modestly inconsistent initial handling conditions (i.e., initial processing and placement at $-80{ }^{\circ} \mathrm{C}$ completed in $<3 \mathrm{hrs}, 3-16 \mathrm{hrs}$, or $>16 \mathrm{hrs}$ ). $\Delta$ S-Cys-Albumin was inversely correlated with pre-centrifugation delay at $4{ }^{\circ} \mathrm{C}$ as well as total delay prior to final storage at $-80^{\circ} \mathrm{C}(\mathrm{p}<0.001)$. Samples from two collection sites had much lower $\Delta$ S-Cys-Albumin values relative to all other sites because they were stored at $-20^{\circ} \mathrm{C}$ (above their freezing point) for an average of 7.6 months prior to shipment to the central repository for final storage at $-80^{\circ} \mathrm{C}$. Based on the rate law for S-Cys-Albumin formation in plasma ex vivo, the average time that each plasma sample was exposed to the equivalent of $23^{\circ} \mathrm{C}$ was back-calculated from the measured $\Delta \mathrm{S}$-CysAlbumin values. The relationship of these results to potentially sensitive clinical analytes will be discussed.

Conclusions: P/S samples from multisite collection studies are vulnerable to a substantial degree of pre-analytical variability. Biochemical verification of specimen integrity is strongly recommended to ensure the integrity of downstream research results.

\section{O-04 Can Genomic ScreenTapes Be Used to Assess Integrity and Concentration of DNA Extracted from Formalin-Fixed, Paraffin-Embedded (FFPE) Tissue?}

\author{
Z. Frazer ${ }^{2}$, N. Guljar ${ }^{2}$, C. Bellora ${ }^{1}$, W. Mathieson ${ }^{1}$ \\ ${ }^{1}$ Integrated Biobank of Luxembourg, Luxembourg, ${ }^{2}$ Imperial \\ College London, London, United Kingdom
}

Background: The Agilent TapeStation is an automated nano-electrophoresis system. A Genomic DNA ScreenTape (GST) runs 15 samples $(1 \mu \mathrm{l})$, returning concentration, sizerange $(0.2-60 \mathrm{kbp})$, and a DNA integrity number (DIN) that ranges from 1 (degraded DNA) to 10 (intact DNA). We validated GSTs using DNA extracted from FFPE tissue.

Methods: GST DNA concentration: compared to Pico Green $(\mathrm{n}=218)$. GST sizing accuracy: $100 \mathrm{bp}$ ladder $(0.1-2 \mathrm{kbp}), \mathrm{kbp}$ extension ladder $(0.5$ - $40 \mathrm{kbp})$, and sample DNA $(\mathrm{n}=15)$ compared with a $1.5 \%$ agarose gel stained with GelRed then analysed by Image Quant software. DIN reproducibility: samples $(n=15)$ applied to six GSTs and the same sample in all 15 lanes of one GST. Shelf life: DINs from three-month date-expired GSTs $(n=3)$ compared to in-date GSTs $(n=3)$, using 15 samples. Influence of RNA contamination on DIN: RNA spiked into DNA at seven concentrations. DIN and DNA functionality: end-point multiplex PCR (100 - 400 bp amplicons visualized by gel electrophoresis, $\mathrm{n}=66$ ) and qPCR (Illumina FFPE QC Assay, $\mathrm{n}=248$ ).
Results: Mean DIN was 5.65 (SD 1.3), range 1.6 - $8.8(\mathrm{n}=461)$. GST DNA concentrations strongly correlated with Pico Green $(\mathrm{x} 2=0.91, \mathrm{p}<0.01)$, with experimental error (median 52\%) slightly increasing with poorer sample purity assessed using 260:230 (x2= $-0.30, \mathrm{p}=0.003$ ). GST sizing accuracy (DNA ladders) was comparable with the agarose gel above $2 \mathrm{kbp}$ (median error $=6.1 \%$ [GST] and $8.3 \%$ [gel], $\mathrm{p}=0.66$ ) but poorer below $2 \mathrm{kbp}$ (median error $=17.2 \%$ [GST] and $1 \%$ [gel], $\mathrm{p}=0.003$ ). GST and gel electropherograms from samples had similar plot-shapes but mean maximum peak size was $510 \%$ higher in GST $(\mathrm{p}<0.001)$. DIN reproducibility: mean CVs were $2.5 \%$ (inter-GST) and $0.7 \%$ (intraGST). RNA contamination had no influence on DIN, nor did using date-expired GSTs. DIN had no correlation with $\mathrm{Cq}$ or $\Delta \mathrm{Cq}$ in the Illumina FFPE QC Assay (x2=0.04, p=0.5).

Conclusions: DNA extracted from FFPE tissue typically falls midway on the DIN scale with a DIN range of sufficient magnitude for meaningful integrity quantification. GSTs have good reproducibility. GST-derived concentrations correlate well with Pico Green but with an average error rate of $52 \%$. DIN does not denote functionality in qPCR, so a combination of DIN and qPCR might offer the best overall assessment of DNA integrity. GSTs are more user-friendly, faster, and require less input DNA than conventional gel electrophoresis but sizeassessment is less precise.

\section{O-05 The Relevance of Preclinical Rodent Samples in Omics Method Development and Understanding of Disease Mechanisms}

\section{S. Ansari, E. Dargaud}

\section{Research Technologies, Philip Morris International - Science} \& Innovation, Neuchatel, Switzerland

Statement of the Problem: For the past 10 years, Philip Morris International (PMI) has conducted numerous preclinical studies on rodents to evaluate the effects of reduced risk products. As a part of PMI's 3Rs strategy for responsible use of animal samples and reducing animal testing, samples that were collected additionally or not consumed for experiments were systematically stored in the in-house biorepository for future analyses. These biospecimens were not only rigorously collected for optimal conservation and reusability but also associated with rich data derived from the preclinical studies, including data on animal health and behavior, body weight, food consumption, histopathological characteristics, cell counts, lung function, and exposure markers as well as various analytics from conventional analytical chemistry to omics endpoints. In addition, a large variety of sample types were collected that included nearly all available organs and specific sections of the organs. The outcome was a repository with more than 200 different sample types and up to 80,000 samples.

Proposed Solution: Today, we often use these samples in analyses to better understand disease mechanisms. Specific rodent strains are accepted as translational models for specific human diseases and, therefore, very commonly used for disease mechanism studies. At the same time, the rapid development of new sample processing methods as well as novel omics technologies requires continuous supply of biological samples. The use of rodent samples in method development is often more appropriate given the ethical concerns over the use of clinical samples. Ultimately, methods are also validated with these rodent samples.

Conclusions: In this work, we will provide a few examples that demonstrate the importance and prominence of these rodent samples and the impact they have had on our clinical study endpoints and general understanding of particular diseases and their mechanistic modelling. 
O-06 Feasibility and Comparison Study of Fecal Sample Collection Methods in Kidney Transplant Recipients using 16S rRNA and Metagenomics Approaches

S. Kurian ${ }^{1,4}$, B. Barrick ${ }^{1,4}$, S. Gordon ${ }^{3}$, J. Nichols ${ }^{3}$, M. Dadlani ${ }^{2}$, C. Marsh ${ }^{1,4}$, J. Case ${ }^{1,4}$

${ }^{1}$ Scripps Clinic Bio-Repository and Bio-Informatics Core, La Jolla, California, United States, ${ }^{2}$ CosmosID, Rockville, Maryland, United States, ${ }^{3}$ Scripps Research, La Jolla, California, United States, ${ }^{4}$ Scripps Center for Organ Transplantation, La Jolla, California, United States

Background: The human microbiome is complex and dynamic comprising a variety of microbes in direct contact with the body. The microbiome plays an important role in health and disease. Interest in the microbiome has surged due to the availability of advanced sequencing that has enabled rapid, accurate, and cost-effective identification of the microbiome. However, barriers remain regarding fecal sample collection, primarily the awkward collection method, and the concerns around hygiene and contamination, leading to compliance rates rarely $>50 \%$ in microbiome studies. In this single-center pilot study, we compared three fecal sample collection methods utilizing 16S rRNA gene sequencing and the comprehensive shotgun metagenomic sequencing.

Methods: Stool was collected from five healthy volunteers and two kidney transplant patients into DNA/RNA shield solution using 1) the standard collection ("scoop") $10 \mathrm{~mL}, 2$ ) rectal swabs in $2 \mathrm{~mL}$, and 3) moist wipes in $15 \mathrm{~mL}$. $200 \mathrm{ul}$ of each sample was taken into the Qiagen DNeasy Powersoil Pro kit for DNA extraction in randomized batches. Samples were quantified for double-stranded DNA via the Qubit HSDNA assay. 16S sequencing was done using the Bioo Scientific NEXTflex 16S V4 Amplicon sequencing kit (2x300bp reads: 5 million reads/sample), and shotgun sequencing using the NEBNext Ultra II DNA Library Prep Kit for Illumina (2x 5bp reads: 20 million reads/sample).

Results: The swab and wipe collections were comparable and reproducible for reads/sample using both sequencing methods when compared to the scoop, which is the current "gold standard" for collection. In one kidney transplant patient, the wipe collection method failed to yield DNA, and was significantly different from the other wipe samples in one volunteer using a Principal Components Analysis plot. The metagenomics analyses, though more granular, was not substantially more informative than the 16 s methodology.

Conclusion: The simpler, more practical swab and wipe stool collection methods were comparable to the relatively cumbersome scoop collection method. However, the swab collection is the preferred method as failure and discordance rates were higher using wipes. 16s sequencing is enough to capture the diversity of the microbiome and is less expensive and more amenable to multiplexing. A longitudinal $16 \mathrm{~s}$ sequencing study of 25 kidney transplant patients is planned and we will utilize the swab and wipe methods to identify patterns of the early post-kidney transplant response.

\section{O-07 Electronic Consent for Pediatric Biobanking: Do Kids and Parents Understand What They Consent To?}

S. Vercauteren ${ }^{1}$, A. Virani ${ }^{2}$, H. Longstaff ${ }^{3}$, J. Robillard ${ }^{2}$, E. Portales-Casamar ${ }^{4}$, A. Lutynski ${ }^{5}$, G. Lei ${ }^{4}$, K. Plain $^{3}$, A. Ellis ${ }^{2}$, M. Dittrick ${ }^{1}$, A. McKerrow ${ }^{4}$

${ }^{1}$ Pathology and Laboratory Medicine, BC Children's Hospital, Vancouver, British Columbia, Canada, ${ }^{2} B C$ Children's
Hospital, Vancouver, British Columbia, Canada, ${ }^{3}$ Provincial Health Services, Vancouver, British Columbia, Canada, ${ }^{4} B C$ Children's Hospital Research Institute, Vancouver, British Columbia, Canada, ${ }^{5}$ Emily Carr Institute, Vancouver, British Columbia, Canada

Background: Pediatric biobanking has several different ethical and legal challenges that differ from adult biobanking. One of those challenges involves informed consent of the parents or legal guardians as well as assenting children to actively include them in the consenting process. The BC Children's Hospital BioBank (BCCHB) is an institutional biobank that collects samples and data from children, infants, and pregnant women. Currently, we have a long and cumbersome paper consent form that is not conducive for children. The objective of this study was to create a child-friendly patient-focused consent platform with input from parents and children.

Methods: We created a prototype for an electronic consent (e-consent) platform. The e-consent is graphic with a childfriendly design to reduce unnecessary text and provide information through multiple methods including video and pictures. A survey was created with a link to the prototype to assess general opinions as well as understanding of the e-consent that was distributed amongst parents, children (7-13 years), and adolescents (14-18 years) of BCCHB participants as well as the BCCH's patient's network. Changes are made based on the received feedback and a focus group is planned to show updated prototype and discuss improvements, identify words/phrases for clarification, discuss practical aspects (timing, presence of BioBank staff), and assess appropriateness of a quiz at the end of consent process.

Results: We received 113 completed survey (adults $n=83$, adolescents $n=24$, and kids $n=6$ ); $57 \%$ had previously consented to the BCCHB. Of all respondents, $41 \%$ stated they preferred the electronic consent form and the same percentage indicated they had no preference between paper and e-consent. Only $16 \%$ stated they preferred a paper consent form. This was a consistent finding among the different age groups. The vast majority of participants stated they understood the purpose of the BioBank, risks, benefits, ability to withdraw, and privacy. Overall, the majority of participants stated the amount of information provided was 'just right.' Free text comments were provided to improve the platform.

Conclusions: Preliminary findings from the development and evaluation of an e-consent platform suggest that this format may be a suitable, efficient, and engaging alternative to paper-based consent for a pediatric biobank and input from participants allows the development of a patient focused consenting method.

\section{O-08 A Road Map for Developing Uganda National Policy Guidelines for Biobanking}

H. Nansumba ${ }^{1}$, S. Nassimbwa ${ }^{2}$, R. Kisame ${ }^{3}$, B. Mutumba ${ }^{4}$, H. Opolot ${ }^{4}$, E. Thomas ${ }^{5}$, I. Ssewanyana ${ }^{1}$, S. Nabadda ${ }^{1,6}$, F. N. Nakwagala ${ }^{7}$

${ }^{1}$ National Biorepository, Uganda National Health Laboratory Services, Kampala, Uganda, ${ }^{2}$ Medical Research Council, Entebbe, Uganda, ${ }^{3}$ Infectious Disease Institute, Kampala, Uganda, ${ }^{4}$ Uganda National Council for Science and Technology, Kampala, Uganda, ${ }^{5}$ Med Biotech Laboratories, Kampala, Uganda, ${ }^{6}$ Ministry of Health, Kampala, Uganda, ${ }^{7}$ Mulago National Referral Hospital, Kampala, Uganda

Introduction: In developing countries, biobanking is advancing as a key pillar in supporting public health, biomedical 
research, and technology within the pharmaceutical, scientific, clinical, and public health sectors. It has facilitated the identification and provision of samples and data required for research, academia, industry, and public health interventions. In Uganda, biobanking presents opportunities for advancing science and technology through local and/or international networking and collaborations. However, this opportunity is challenged by the inadequacy of the relevant ethical, legal, and regulatory guidelines within the country. The aim of this abstract is to discuss the road map and steps undertaken for developing the National Policy Guidelines for Biobanking.

Objectives of the Guidelines: 1) To ensure that biobanks are established for the custodianship of high-quality, highly valued biological materials and data; 2) to maintain legal standards in management of biobanks; 3) to maintain ethical standards in collection, processing, storage or inventory, disposal, use, and distribution of biological material; and 4) to maintain biosafety and biosecurity standards in collection, processing, storage or inventory, disposal, use, and distribution of biological material.

Methodology: Appointment of Technical Working Group (TWG) by Uganda National Council for Science and Technology (UNCST): A TWG was formed by UNCST to develop the National Bio-banking Guidelines. Participants of the Technical Working Group included a whole range of stakeholders: laboratory technical personnel, epidemiologists, lawyers, microbiologists, ethicists, and community and policy makers. Five phased meetings have been scheduled; the first three meetings aimed to write and review of the National Biobanking Guidelines. The fourth meeting shall be a national consultative meeting and lastly the dissemination.

Discussion: One of the topics for further debate from the TWG meeting deliberations was the benefit sharing and intellectual property. This is in relation to biological materials and data that have been shipped out of Uganda. There is a weak international regulatory framework and monitoring mechanisms of third-party use. In many scenarios, requirements of the Material Transfer Agreements may not be adhered to by researchers in both local and international collaborations.

\section{O-09 Returning Value to Participants in Biobanking and Research: The Cancer Moonshot Biobank Patient and Provider Engagement Portal}

E. Casas-Silva ${ }^{1}$, H. Ellis ${ }^{1}$, V. Gopalakrishnan ${ }^{1}$, A. Rao ${ }^{1}$, L. Agrawal ${ }^{1}$, P. Guan ${ }^{1}$, C. Weil ${ }^{1}$, N. Madero ${ }^{2}$, J. McClean $^{2}$, S. McDermott ${ }^{2}$, J. Suh ${ }^{2}$, M. Jensen ${ }^{2}$, J. Wanyiri ${ }^{2}$, M. Williams ${ }^{2}$, H. Moore ${ }^{1}$

${ }^{1}$ National Cancer Institute, Rockville, Maryland, United States, ${ }^{2}$ Leidos Biomedical Research, Frederick, Maryland, United States

Statement of the Problem: Biospecimen donation is quintessential to successful biobanking and research. Despite evidence demonstrating a clear reciprocal benefit to engaging the public and health providers as partners in biobanking, there is still much room for improvement. Challenges include lack of resources as well as strategies from which to learn and adapt.

Proposed Solution: The National Cancer Institute's (NCI) Cancer Moonshot Biobank is a new 5-year project with the aim of engaging a diverse group of participants with advanced cancers in biospecimen and data donation throughout the course of their cancer treatment. Patients are being recruited through participating NCI Community Oncology Research Program
(NCORP) sites. The ultimate aim of the Biobank is to accelerate research on the two main causes of cancer deaths: treatment resistance and the spread of cancer in the body (metastasis).

A central tenet of the Biobank will be to return value to patients and provider participants by interfacing with them directly. As such, the Biobank has instituted three main components of engagement:

1) External Scientific Panel: An extramural team of physicians, patient advocates, communication, and biobanking experts who give input on various aspects of Biobank engagement

2) Engagement programs at Biospecimen Source Sites

3) Patient and Provider Engagement (PPE) portal

The PPE portal was designed for direct interface between the Biobank and its participants. Through the Portal, participants can see program updates, download documents such as clinical biomarker tests and signed consent forms, and access educational material. The Portal will serve as an archetype within NCI for its current development of a unified portal for research participant engagement projects. Here we describe ideation, design, and content creation for the Cancer Moonshot Biobank PPE portal. Strategies for obtaining public and expert feedback, procedures for design and content iteration, as well as lessons learned will be discussed.

Conclusions: The goal of the Cancer Moonshot Biobank Patient and Provider Engagement portal is to serve as a point of connection between the Biobank and its participants. Through its creation we aim to increase patient and provider engagement in biobanking and research by providing an archetype that could ease adoption of online engagement strategies within NCI and beyond.

\section{0-10 Policy Innovation and Tribal-Federal Partnership: The Alaska Area Specimen Bank}

B. Simons-Petrusa ${ }^{1}$, T. Woods ${ }^{2}$, A. Willetto ${ }^{2}$, T. Thomas ${ }^{2}$, J. Beans ${ }^{3}$, J. Klejka ${ }^{4}$, C. DeByle ${ }^{1}$, M. Bruce ${ }^{1}$

${ }^{1}$ Centers for Disease Control and Prevention, Anchorage, Alaska, United States, ${ }^{2}$ Community Health Services, Alaska Native Tribal Health Consortium, Anchorage, Alaska, United States, ${ }^{3}$ Southcentral Foundation, Anchorage, Alaska, United States, ${ }^{4}$ Yukon Kuskokwim Health Corporation, Bethel, Alaska, United States

The Alaska Area Specimen Bank (AASB) has operated for 20 years as a tribal-federal partnership that co-manages the biorepository. The mission of the AASB is to provide a resource for research focused on the health of people in the Arctic, especially indigenous communities. The written policies of the AASB partnership have successfully guided utilization of this historic and invaluable resource.

Statement of Problem: As technologies advance and nuances of confidentiality and ownership arise, the AASB partnership has determined that the current policies require revision and amendment.

Proposed Solution: Through the unique communications structure of the AASB partnership, a revisions committee has been established with broad representation and a defined process for developing a policy that meets the needs for both tribal and federal partners. The process: 1) review of best practices, including specific guidelines from Alaska Native and American Indian resources; 2) summarize and identify key themes and elements from all reviewed resources; 3) evaluation of current AASB policy document and identification of missing elements; 
4) prioritize revisions and convene meeting for consensus and definition of terms for the revised policy; and 5) revise the AASB policy and submit for approval by all tribal and federal partner entities.

Conclusion: The revisions committee identified and summarized key themes from best practice resources and has reviewed with the AASB partnership. An evaluation of the current AASB policy has begun, identifying several opportunities for inclusion of best practices in the AASB policy that also meet the needs of this unique tribal-federal partnership. A two-day workshop in 2020 has been designed to review proposed revisions and approve by consensus amongst all tribal and federal partners.

\section{0-11 Creating and Refining Policy for Sample and Data Sharing in Southern African Biorepositories}

E. S. Mayne ${ }^{1}$, M. Kader ${ }^{2}$, G. Swartz ${ }^{2}$, T. Moshoma ${ }^{2}$

${ }^{1}$ Immunology, Faculty of Health Sciences, University of Witwatersrand and National Health Laboratory Service, Johannesburg, Gauteng, South Africa, ${ }^{2}$ Clinical Laboratory Services, Wits Health Consortium, Johannesburg, South Africa

Statement of Problem: Biorepository Science is a relatively novel field in the African arena. A number of different African institutions have established infrastructure to store human biological material for future research purposes but in many cases, it is difficult to interpret the legal and ethical frameworks which should inform biorepository governance. In some cases, this can result in challenges in ensuring that biorepositories conform to the appropriate in-country standards while supporting appropriate usage of both human biospecimens and data. In South Africa, a number of laws and guidelines refer obliquely to biorepository storage for research purposes, including the National Health Act (Act 12 of 2013) and the Protection of Personal Information Act (Act 4 of 2013), but these refer primarily to storage for therapeutic purposes (tissue banking). They provide insufficient guidance on a number of key aspects including generation of material transfer agreements, benefit sharing, research on underserved or vulnerable populations, and how data can be transferred to researchers without impinging on participant confidentiality.

Proposed Solution: This study proposes to utilise the regulations and legal framework in South Africa as a test case to identify gaps where guidelines and best biorepository practice can be utilised to supplement existing legislation. In doing this, it will engage with governmental and academic bodies to facilitate a shared understanding of the requirements of operating a research-intensive biorepository within the African setting.

Conclusion: Biorepository Science in Africa has a unique opportunity to liaise with governmental and non-governmental stakeholders to ensure that legislation and ethical guidance is informed by biorepository best practice.

\section{O-12 Aspects of Biobanking Governance: Legal or Ethics?}

\section{H. J. Silverman}

School of Medicine, University of Maryland Baltimore, Baltimore, Maryland, United States

Statement of the Problem: The recent proliferation of biobanks has led to discussions on how best to regulate these entities. The discourse regarding governance has centered predominantly on legal aspects that might neglect a vigorous dis- cussion on the ethics of this new "genetic" revolution. This paper attempts to explore the ethical issues at both the individual and societal levels and concludes with the claim that ethics should occupy a central place in the current discourse on governance.

Proposed Solution and Conclusions: Obtaining biological samples is viewed as a minimal risk activity with the prospects of new breakthroughs in genetic discoveries. This seemingly low risk/great benefit activity belies the ethical complexities of biobanking that makes clear the potential for enormous risks.

At the individual levels, should the type of informed consent be based on what appears to be a consensus towards "broad consent" or does tiered consent reflect better the concern for individual control of biological samples? Does the present consensus of ownership and commercial profits that neglect the individual withstand vigorous ethical analysis? What are the implications of biobanking on privacy, self-determination, and individual discrimination? What will be the effects of commodification on personal identity and integrity? Will the emphasis on genetic determinism coupled with development of preventative measures lead to unrealistic notions of selfresponsibility? Will talk of genetic solidarity and altruism compel individuals to donate their biological samples or shame those who do not?

Unanswered ethical complexities occur at the societal level. For example, from where will funding occur to support the substantial investment needed for biobanks? Will the huge capital and human resources needed to support biobanking leave the low- and middle-income countries behind? If substantial public funding occurs without immediate health benefits, will the ensuring public distrust lead to a collapse of biobanking activities? Will biobanking efforts exacerbate social inequalities and enhance discrimination based on genetic differences? Will there be honest attempts at community engagement and consultation? Will data-privacy laws be overly restrictive?

In conclusion, a discourse regarding governance should be guided by vigorous ethical analysis to ensure that biobanking integrates successfully in the fabric of society and informs legal suppositions.

\section{O-13 New Biobanks Require New Generation of Biobankers: Experience from the Czech Republic}

\section{J. Kinkorova}

Department of Immunochemistry, University Hospital in Pilsen, Pilsen, Czechia

Statement of the Problem: Biobanking is a new field of personalised medicine approach to the patient health care with innovative potential. As hundreds of well-established biobanks currently operate worldwide, new biobanks are established. Biobanks are generally based on samples and associated data, and biobanking as a rapidly developing science is multidisciplinary, requiring clinicians, surgery doctors, laboratory staff, pathologists, and imaging specialists, and, on the other hand, information technology (IT) specialists, statisticians, and data and big data specialists. To fully and reliably operate a biobank, a new generation of biobankers is needed.

Proposed Solution and Conclusions: At University Hospital in Pilsen and closely related Faculty of Medicine in Pilsen, Charles University, together with University of West Bohemia, special courses are performed in immunoanalytical methods, and clinical applications, biobanking, and personalised medicine. Biobanking education is conducted also at the international level. In the frame of bilateral project of cross-border collabo- 
ration between Bavaria (Germany) and Pilsen (Czech Republic) BRoTHER (Biobank Research on Telemedical Approaches for Human Biobanks in a European Region), several types of activities were done, including summer school with main biobanking issues such as: ELSI; grant applications in biobanking, sustainability of biobanks, IT in biobanks, preanalytics, and research in biobanks; and student exchange was realised by the Bavarian partner. On the other hand, an intensive course in immunochemistry, biobanking, and personalised medicine was organised by the Czech partner for both German and Czech students. Based on very good experience, new activities as well as new curricula will be prepared.

Another activity is the establishment of a group of young scientists all over the word including biobankers under the umbrella of EPMA (European Association for Preventive, Predictive and Personalized Medicine). The concept of education of young scientists includes introducing young researchers in the field of predictive, preventive, and personalised medicine; involving them in national and international projects; and creating an international network to promote collaborations between young scientists, universities, and research laboratories. Experience obtained at national and international levels will help to educate the next generation of biobankers.

\section{O-14 Biobanks Supporting Clinical Study Submission - Clinical Data Interchange Standards Consortium (CDISC) Standards for Biospecimens}

O. Karch ${ }^{1}$, C. Connolly ${ }^{2}$, A. Kruljac-Letunic ${ }^{1}$

${ }^{1}$ Clinical Biomarker Informatics \& Biobanking, Merck KGaA, Darmstadt, Germany, ${ }^{2}$ EMD Serono, Rockland, Massachusetts, United States

Biobank data standards, such as MIABIS (Minimum Information About BIobank data Sharing), greatly facilitate exchange of specimen information among different organizations and biobank directories. However, when banked samples are released in the context of clinical precision medicine studies (e.g., to develop assays to support Companion Diagnostics), study results are typically required to be submitted to regulatory authorities in CDISC Study Data Tabulation Model (SDTM) format. While the SDTM biospecimen domains are provisionally published in the SDTM Implementation Guide for Pharmacogenomics and Pharmacogenetics (PGx) Version 1.0 (SDTMIG-PGx v1.0), it is acknowledged that these domains could potentially serve broad submission and industry use-cases beyond PGx. Currently, the focus of SDTM domains for biospecimens is geared towards provenance of samples used for laboratory testing supporting sponsor oversight, Good Clinical Practice, and other GxP processes. Consequently, these standards support detailed specification of:

- Related Specimens to describe sample genealogy and parentchild specimen relationships

- Biospecimen Events to capture biospecimen logistics; e.g., collection, transport, freeze/thaw cycles, temperature excursions

- Biospecimen Findings to record characteristics of biospecimens; e.g., volume and integrity of the DNA or RNA samples

Recently a team within CDISC formed to evaluate current provisional SDTM standards for both submission and broad industry use cases. By aligning with SDTM representation of biospecimen-related data, it may be possible to support both regulatory submission packages and tracking of biospecimens within a biobank. Example use cases will be presented to demonstrate utility of SDTM biospecimen domains for regulatory submission and operational activities.

\section{O-15 Redeveloping a Human Tissue Retrospective Research Project Request Form}

T. H. Shaw, B. Sharp, M. H. Cessna, C. Sainsbury, D. Wilcox, D. K. Crockett

Biorepository, Intermountain Healthcare, South Salt Lake City, Utah, United States

Background: Retrospective research project intake can be a lengthy process involving multiple clarification cycles between the biorepository and researcher to resolve key project requisites. Our initial one-page project request form (PRF) used vague, open-ended questions that inadequately captured vital details required to release archived human tissue samples. Crucial project edits were buried in various places leading to various oversights during project execution.

Methods: We began by identifying our most frequent followup questions after receiving PRFs and evaluating similar intake forms from related industries. We then coalesced questions that applied to the majority of projects and samples; however, we also needed specific questions on selective areas (e.g., oncology studies). Due to the complexity and variety of topics, we chose REDCap for its branching logic capability. For any open-ended free-text fields, we provided several example responses to help target responses. At the end of the drafting process, we solicited input from both internal and external stakeholders.

Results: After a three month redevelopment process, we implemented the new PRF in 02/2018. Since implementation, over 40 PRFs have been submitted. The PRF transformed from 20 ambiguous questions to a dynamic form containing $250+$ logic-based fields (25\% structured $/ 75 \%$ free-text) arranged in following topics: project details (including study population); required samples and data; regulatory approvals; timeline; and information about shipping, funding, and invoicing. The PRF guides researchers through relevant sections that only pertain to their project. Since implementing the revised PRF, our project intake has improved significantly by obtaining essential project details early on. In addition, the PRF is a living document that can be modified easily with any vital project changes and serves as a single source of truth throughout the project lifecycle.

Conclusions: A robust PRF leads to an efficient intake process by encouraging researchers to define project and sample requirements before submitting a request, which reduces unnecessary communication cycles and clarification requests. By organizing the key project details in one location, a comprehensive PRF enables biorepositories to effectively support projects and successfully facilitates the entire project lifecycle.

\section{O-16 Data Management Tools in the NIST Biorepository}

\section{J. M. Ragland, A. Moors, R. Pugh}

Chemical Sciences Division, National Institute of Standards and Technology, Charleston, South Carolina, United States

Asset management in biorepositories has historically been focused on capital assets such as facilities, staff, and freezers. As technical data management tools have improved, data have increasingly become considered an asset to the same degree. 
Such data can describe the facility, a collection, or any downstream data produced. Breadth and focus can range from passthrough facilities holding minimal data describing samples and their storage, to facilities attached to clinical laboratories where analytical tests are ordered and the results stored. Regardless of the type of data associated with physical collections, ensuring the highest possible data quality is of utmost importance to achieving operational excellence. Commercial software targeting biorepositories helps tremendously and is generally flexible enough to fit the broad array of biorepositories. It cannot, however, fully substitute for robust data quality management and practices; there will always be a gap between data management tool designs and the practical needs of and direct implementation within any given repository. Over the last few years, the NIST Biorepository in Charleston, SC, has developed several data-first tools and approaches to specifically address this gap with regard to data quality management. These include 1) data collection tools that capture data more stringently from field or collaborator collection events; 2) workflow curation tools to ensure each process occurs in the same way every time, regardless of how much time has passed since the last event needing that workflow; 3) data quality audit practices; 4) data visualization tools to manage capital assets such as freezers; and 5) a tool to conduct physical position audits in a rapid manner. Combined, these tools and associated approaches have greatly reduced data entry errors, accelerated time-intense data quality activities, and provided at-a-glance insights into the function and status of the biorepository. The general approach, as well as discrete benefits and reasons for the creation of five specific tools, will be discussed. Custom data tools such as these are currently saving the NIST Biorepository over 1,000 hours of staff time annually and highlight the necessity of a technical data manager in modern day biorepositories. This presentation is targeted toward repository managers, data managers, and those interested in improving data quality practices and streamlining biorepository operations.

\section{O-17 An Economic Analysis of Cancer Biobanks in New South Wales}

A. Rush ${ }^{1}$, D. R. Catchpoole ${ }^{2}$, R. Ling ${ }^{3}$, A. Searles ${ }^{3}$, P. Watson ${ }^{4}$, J. Byrne ${ }^{5}$

${ }^{1}$ The Children's Hospital at Westmead Clinical School, University of Sydney, Sydney, New South Wales, Australia, ${ }^{2}$ The Children's Hospital at Westmead, Sydney, New South Wales, Australia, ${ }^{3}$ Hunter Medical Research Institute, Newcastle, New South Wales, Australia, ${ }^{4}$ BC Cancer, Vancouver, British Columbia, Canada, ${ }^{5}$ NSW Health Statewide Biobank, Sydney, New South Wales, Australia

Background: Despite the recognized importance of biobanking, sustainability challenges are common, and there are few published comparisons of biobank costs and outputs. We hypothesize that a lack of output data, and their value, represent threats to biobank sustainability. Our project aims to determine the costs, investments, and outputs of a cohort of Australian cancer biobanks, to provide explicit and accountable economic data to all biobank stakeholders.

Methods: An interview template was developed and invitations were emailed to 17 Australian cancer biobanks, of which 12 biobanks participated (open-access: $n=6$; restricted-access: $n=6$ ). Ratios of monetary to in-kind contributions, numbers of publications and clinical trials supported, and costs per supported publication were compared according to biobank access policy, funding sources, and biobank size, during a defined reporting period (2015-2018).

Results: Analyses ( $n=6$ open-access; $n=6$ restricted-access) show that ratios of monetary to in-kind support were higher for the open-access biobanks (8:1) than for the restricted-access biobank (5:1). Open-access biobanks supported more total research publications than restricted-access biobanks $(n=62$ versus $\mathrm{n}=46)$, and had greater median publications per biobank (12, range $0-22$ versus 7.5 , range $4-11$ ). Median costs per publication (USD $\$ 25,500$ versus USD $\$ 25,600$ ) and median journal impact factors (4.90 versus 5.17) were comparable. Open-access biobanks had a greater rate of authorship on publications when compared with restricted-access biobanks (65\% versus 54\%).

Conclusions: Comparisons of biobank costs and outputs can provide useful measures of biobank value, and could also illuminate differences in the operations and contributions of openand restricted-access cancer biobanks. For examples, institutions and their staff may be more willing to provide in-kind support to biobanks with local outputs. Comparable median journal impact factors and costs per publication suggests that access-policy is not a factor in determining publication quality, or overall costs per publication. These data can inform biobanks and their stakeholders on sustainability challenges.

\section{O-18 Moving with the Times: The Health Science Alliance Biobank Pathway to Sustainability}

C. M. Quinn ${ }^{1}$, M. Porwal ${ }^{1}$, N. S. Meagher ${ }^{2,3}$, A. Hettiaratchi ${ }^{4}$, W. Liauw ${ }^{5,6}$, S. Macmillan ${ }^{1}$, C. A. Power ${ }^{7}$, S. McCullough ${ }^{8}$, D. Goldstein ${ }^{1,3}$, N. Zeps ${ }^{9,10}$, P. J. Crowe ${ }^{3,11}$

${ }^{1}$ Translational Cancer Research Network, University of New South Wales, Sydney, New South Wales, Australia, ${ }^{2}$ School of Women and Children's Health, University of New South Wales, Sydney, New South Wales, Australia, ${ }^{3}$ Prince of Wales Clinical School, University of New South Wales, Sydney, New South Wales, Australia, ${ }^{4}$ UNSW Biorepository, University of New South Wales, Sydney, New South Wales, Australia, ${ }^{5}$ Cancer Services Stream, South Eastern Sydney Local Health District, Sydney, New South Wales, Australia, ${ }^{6}$ St George and Sutherland Clinical School, University of New South Wales, Sydney, New South Wales, Australia, ${ }^{7}$ Biological Resources Imaging Laboratory, University of New South Wales, Sydney, New South Wales, Australia, ${ }^{8}$ Consumer Advisory Panel, Translational Cancer Research Network, University of New South Wales, Sydney, New South Wales, Australia, ${ }^{9}$ Epworth Healthcare, Melbourne, Victoria, Australia, ${ }^{10}$ Eastern Health Clinical School, Monash University, Melbourne, Victoria, Australia, ${ }^{11}$ Department of Surgery, Prince of Wales Hospital, Sydney, New South Wales, Australia

Statement of the Problem: Following a global trend, the last decade has seen the development of several Australian poly-user model biobanks, wherein biospecimens are collected prospectively in anticipation that researchers will use them. The Health Science Alliance (HSA) Biobank is a cancer-specific biobank located in South-Eastern Sydney that was established in 2012 to collect biospecimens from all tumour types. Currently the HSA Biobank has an array of high-quality biospecimens from 3,500 people with broad consent permitting linkage of clinical and outcome data. The biobank has directly supported 32 projects (30 since 2016) and resulted in 40 publications and conference presentations.

As recognised by biobanks internationally, the HSA Biobank must adapt to changing research needs in order to maximise utility and ensure sustainability. While demand for the HSA Biobank resources continues, there are bespoke research re- 
quirements that cannot be accommodated by the generic collection model.

Proposed solution: The continuing collection and annotation of biospecimens remains at the core of HSA Biobank activity, and hospital-embedded biobanking infrastructure allows the HSA Biobank to retain this 'biospecimen-centric' approach. However, due to broader, investigator-initiated definition of what constitutes a biospecimen, non-routine 'investigator-centric' collection is needed in parallel. The following four areas will test a hybrid model, with pilot studies underway.

1. Organoids, explants, and live cell culture: a process is being trialled for researchers to directly access tissue from surgery for the creation of models involving live cells.

2. Microbiome specimen banking: collection of stool and oral swab specimens is underway, allowing research into gut and oral microbiota in different cancers and at different stages of treatment.

3. Bioimage banking: a proof of principle study for extraction of hospital radiology report data has been completed and expansion to include images is under development.

4. Research data banking: Clinical and survival data along with medical service and pharmaceutical use from upper and lower gastrointestinal cancer patients is being used for health service utilisation research.

Conclusions: Like other biobanks globally, the HSA Biobank is faced with issues of relevance and sustainability. While maintaining its current model, it is also expanding into new areas; relative success of ongoing pilot studies will guide future resource investment.

\section{0-19 The Women First Clinical Trial Biobank: Participants in Vulnerable and Underserved Populations}

J. Kemp, J. E. Westcott, A. E. Hendricks, K. M. Hambidge, N. F. Krebs, W. Nutrition Trial Group

\section{University of Colorado Denver, Aurora, Colorado, United States}

Background: The Women First Maternal Preconception Nutrition Trial is a randomized control trial in four sites located in India, Guatemala, Pakistan, and Democratic Republic of the Congo. Participants include vulnerable and underserved women of reproductive age and their offspring who are at risk of nutrient deficiencies that can have lasting health effects. The Women First clinical trial biobank contains biospecimens collected from these women and their offspring.

Methods: Biospecimens were collected longitudinally from a subset of mothers and their infants at each location. Sample types include blood spot cards, serum, plasma, red blood cells, urine, stool, buccal swabs, birth tissues, cord blood serum, saliva, and breast milk. To ensure sample integrity, we employed standard operating procedures for biospecimen collection and transport that were adapted to the capabilities of each field site. Hemoglobin was measured in the field at all sites. We developed an efficient and low-cost system for printing barcoded cryogenic labels, and tracked biospecimens using the TrackVia platform. TrackVia is a highly customizable system that links biospecimen information with results from that sample. Statistical and data analyses were conducted using $\mathrm{R}$ version 3.5.1.

Results: All biospecimens have been successfully transported and received at the Women First clinical trial biobank located in Denver, CO. Analyses of multiple nutritional and inflammatory biomarkers are almost complete and the data are currently being analyzed. We developed a reproducible quality control pipeline to assess data quality, and to guide further data analyses. Data quality assessments include comparing biomarker distributions, and missing data counts by run, assay date, site, arm, and timepoint as well as identifying outliers, evaluating balanced design, and assessing normality.

Conclusions: Over 45,000 biospecimens with different collection and handling requirements were successfully collected and stored in four low-resource settings, then transported to the biobank. The systems in place at the biorepository have effectively managed the entire life cycle of the samples, linking sample information with results. The capacity and knowledge gained can be applied to future studies with limited resources. The results from this study will support future recommendations on how to further support women and children in these vulnerable communities.

\section{O-20 Feasibility of Assessing Fresh Tumor Quality by Real-Time Remote Digital Viewing Via iPhones: A Study of 195 Cases}

\section{T. J. Bocklage, D. Napier, B. Hallahan, L. Sims}

\section{Department of Pathology, University of Kentucky COM, Lexington, Kentucky, United States}

Background: Quality assessment of fresh human tumor in real-time can confirm both tumor presence and evaluate tumor necrosis. In a prior ISBER platform presentation, we reported that touch preparations or immediate smears can be used to confirm tumor presence/absence and absence/presence of marked (>50\%) necrosis. Touch preparations are rapid, do not consume sparse sample, and can be performed without disrupting the clinical work in the Pathology Grossing Suite. In this follow-up study, we asked whether the same type of sample could be prepared by a biobank technologist and read remotely at any location by a cytopathologist equipped with an iPhone using FaceTime to access in real-time the microscopic features of the smear.

Methods: Over a period of approximate 15 months, two biobank technologists (BTs) created immediate smears of 195 human tumors from patients consented for collection for research. The sample was first selected by a gross room professional such as a pathology resident or pathologist's assistant. The biobanker making the smear had first been trained by a cytopathologist and, as needed, had real-time access to a standard operating procedure and training video. The specimen was gently scraped, deposited on a slide, smeared with a spreader slide, and both slides were stained using a rehydrated hematoxylin and eosin method. Subsequently, the BT transmitted the live microscope image to the recipient pathologist, using a device that coupled the BT's iPhone to the microscope. Once connected via FaceTime, the cytopathologist then directed the BT to move the slide and change the magnification as needed until able to verbally communicate the following: Slide Quality Acceptable/Non-Acceptable; Tumor Present/Absent; Percent Necrosis. Subsequently, the 195 tumor specimens were processed into FFPE blocks, stained with hematoxylin and eosin, and reviewed by the same cytopathologist for the same parameters but blinded to the initial assessments made on the smears.

Results: Seventeen sites were sampled and included predominantly carcinomas (lung, endometrial, ovary, colon most common) but also benign tumors and sarcomas. Accuracy for assessing tumor presence was $90 \%$ and for detecting $<50 \%$ necrosis was $88 \%$. Specificity and sensitivity for tumor presence was $98 \%$ and $50 \%$ and for $<50 \%$ necrosis was $88 \%$ and $100 \%$. 
Conclusions: This initial feasibility study indicates that realtime remote assessment of tumor smears is accurate and specific using a simple set-up with minimal trainnig.

\section{O-21 Construction and Development of Clinical Biological Resource Bank for Women and Children}

X. $\mathrm{Gu}^{1,2}$, J. $\mathrm{Li}^{1}$, L. $\mathrm{Pi}^{1}$, D. Che ${ }^{1}$, L. Fu ${ }^{1}$, Y. Xu ${ }^{1}$, H. Zhou ${ }^{1}$

${ }^{1}$ Department of Clinical Biological Resource Bank, Guangzhou Women and Children's Medical Center, Guangzhou, China,

${ }^{2}$ Department of Blood Transfusion, Guangzhou Women

and Children's Medical Center, Guangzhou, China

Background: Guangzhou Women and Children's Medical Center is the largest women and children's hospital in south China. With the development of translational medicine research and high-throughput biotechnology, the quality and quantity of well-annotated human clinical samples are becoming more and more important. Our hospital established "Clinical Biological Resource Bank" in 2015. To date, our biobank was stocked in more than 1.2 million samples and stocked out almost 100,000 samples for scientific research.

Methods and Results: We established a population-based sub-biobank, by collecting human biospecimens including plasma, serum, cell pellet, and tissues from the samples which had been tested routinely by laboratory or pathological department. Based on gynecological and pediatric diseases, we also established a disease-based sub-biobank for 33 common disease cohorts and 29 major disease cohorts, such as the first biliary atresia cohort in the world, multicenter neuroblastoma cohort, and Kawasaki disease cohort. For some specific cohorts, we collected specimens at different stages of the disease progression.

For the convenience of the biospecimens management, we set a special number for each cohort and specific scientific research barcodes for a variety of specimen types in our hospital information system. We have developed an excellent connection between the biobank information system and hospital information system, such as laboratory, imaging, pathological, nursing, operation, anesthesia system, and so on.

Our biobank has made a great contribution to the scientific research of translational medicine these years. Up to now, it has provided a large number of clinical samples for more than 30 scientific research projects. More than 100 articles have been published in domestic and foreign journals. Our biobank is now playing an increasingly important role in studying molecular mechanisms, identifying risk factors, and developing personalized drug therapies for pediatric and gynecological diseases.

Perspectives: We have been starting to construct a 5 millionlevel automatic $-80^{\circ} \mathrm{C}$ ultra-low and liquid nitrogen bank and upgrade the software intelligent system. We hope that we can select samples smartly according to researchers' needs, and integrate relevant clinical data when stocking out biospecimens from the biobank. These will greatly improve the efficiency and accuracy of in and out of the biospecimens in the coming year.

\section{0-22 Analysis of Sample Integrity for Developing a "Value Statement" of Samples and Biobank in China}

C. Wang, W. Liang, L. Zhao, H. Shan

Xinuha Biobank, Shanghai Xinhua Hospital, Shanghai, China

Statement of the Problem: We usually review work efficiency of biobanking activities by performing rituals report with showing the number of samples and/or cases collected. We are thus excited to hallmark development status by increasingly storing biospecimen at our biobanks. However, when we give some thoughts about it, we realize that such updates do not really mean much to us. Do we know the sample usability? Do we know how many samples can meet our needs to address a scientific question? Instead, we may be disappointed after we get deep into it by searching to retrieve a sample with a specific requirement. Besides, overall utilization of samples is relatively low at most of the biobanks. Taken together, it strongly suggests that we need a different way to review and evaluate collection updates and the values of biobanks.

Proposed Solution: We develop a strategic method using two cohort collections (Shanghai Birth Cohort and Early Life Plan) at Xinhua Biobank. We apply "sample integrity" as the concept to determine the values of collection. We define and evaluate with a focus on three key factors: completeness of associated key elements with samples, the accuracy of the relevant elements, and the consistency of related elements. As we know, sample integrity is the base for sample usability to increase utilization of samples, which can be used to batch or group samples for addressing scientific questions. We thus name it "value statement" of biobank. The key steps involved in the strategy: 1) Define research direction or focus for which we need samples, 2) identify the elements (by associated data, for example, metadata of sample) to determine sample usability, 3) determine their association of the elements (use the three basic boolean operators: AND, OR, and NOT), 4) search the biobank to retrieve the samples that match the requirements defined above.

Conclusions: By applying the method, we have identified some big issues in our cohort collections. Without such "sample integrity" analysis, we are not able to realize these issues in a timely manner. In a single word, by doing so, we believe it would be very useful to make sample collection more useful and usable to increase sample and data utilization. Since a biobank is very costly, it would be critical to identify any issues that reduce sample integrity and correct them to stop wrongdoing of biobanking activities.

\section{O-23 CAP Biorepository Accreditation and ISO 20387: A Gap Analysis}

E. Knutson ${ }^{1}$, J. Rose ${ }^{2}$, S. J. McCall ${ }^{1}$

${ }^{1}$ Department of Pathology, Duke University, Durham, North Carolina, United States, ${ }^{2}$ College of American Pathologists, Chicago, Illinois, United States

Background: As of 2019, there are two third-party accreditation programs available for biorepositories. The College of American Pathologists (CAP) launched its Biorepository Accreditation Program in 2012. In this program, checklist items aligned with CAP Standards of Accreditation are utilized. The International Standards Organization (ISO) published its 20387 standard for biobanking in 2018. To our knowledge, a gap analysis comparing the requirements of the two programs has not previously been performed.

Methods: The ISO 20387 standard was examined and instances of requirements using the word "shall" were identified. The 2019 CAP Biorepository and Lab General checklists were searched for items that addressed each ISO requirement. Missing items were counted as "CAP gaps." Likewise, the ISO 20,387 document was searched for each checklist requirements in the CAP Biorepository and Lab General checklists. Missing items were counted as "ISO gaps."

Results: Out of 166 requirements in the ISO document, 25 CAP gaps were identified. There were multiple gaps around the 
concepts of impartiality and complaint monitoring. Other CAP gaps included financial planning and documented planning for physical or data expansions. Out of 300 CAP requirements, 144 ISO gaps were identified. Many ISO gaps centered around fire, chemical, and biological safety, as well as histology and nucleic acid extraction, which may have simply been out of scope for the ISO document. A few relevant ISO gaps included the CAP requirement for continuing education for biorepository staff and histologic review of tissue specimens. Importantly, both programs require similar overarching quality management programs, personnel training, and competency assessment.

Conclusions: CAP has a higher number of requirements and includes specific laboratory safety and process-specific items. By contrast, the ISO standard is tightly focused on core biorepository activities. When focused strictly on core biorepository activities, there is a high degree of overlap in the programs. Relevant CAP gaps may include the concepts of impartiality and financial planning. Relevant ISO gaps may include a continuing education program for biorepository staff and histologic review of tissue specimens.

\section{0-24 Integration of Biobanks into Precision Medicine - A Real Life Example}

S. K. Petrillo ${ }^{1}$, A. Lazaris ${ }^{1}$, A. Salman ${ }^{1,2}$, P. Metrakos ${ }^{1,2}$

${ }^{1}$ Research Institute of the McGill University Health Centre, Montréal, Québec, Canada, ${ }^{2}$ McGill University, Montréal, Québec, Canada

At the Research Institute of the McGill University Health Centre (RI-MUHC), we have built a research-driven Liver Disease Biobank with the goal to support translational and patient-oriented research. Therefore we must have a dynamic system to respond to those needs, while maintaining all ethical requirements. A biobank not only provides retrospective samples but also, with the evolution of technologies, must have flexible processes in collecting prospective specimens. Furthermore, biobanking activities must be in line with the clinical management of patients, ensuring that sampling methods are seamlessly integrated. With the evolution of technology and precision care, biobanks must be cognisant of new technology developments, such as liquid biopsies, and put in place standard operating procedures for the collection and processing of these samples. For the success of precision medicine, samples must be collected prospectively at pre-determined time points to ensure proper tracking of patients' disease progression and response to treatment.

We have put in place a structure were our workflow allows us to follow participants, similar to clinical trial designs. Participants are monitored and serially sampled and the samples are annotated (i.e., disease status, clinical blood markers, imaging etc.) in a consistent fashion. This has allowed us to integrate our Liver Disease Biobank into large-scale funded precision care initiatives. For example, our biobank is at the core of a funded grant on Precision Care for Colorectal Cancer Liver Metastases. This program is focused on collecting fresh tissue for transcriptome profiling (i.e., single-cell RNA analysis) and also extracellular vesicle isolations, from serially collected plasma samples. The outcomes of this initiative will not only lead to new discoveries but also establish a biomarker signature of clinical relevance.

As a biobank we must think ahead about integration of scientific findings into the clinic and by managing the collection of samples as clinical trials we are one step closer to supporting the implementation of discoveries toward patient care.

\section{0-25 Cryopreservation and Cryobanking of Reproductive Cells of Aquatic Species and its Applications in Biomedicine, Conservation, and Aquaculture}

\section{T. Zhang}

\section{Bournemouth University, Poole, United Kingdom}

Successful cryopreservation and cryobanking of reproductive cells of aquatic species offers many benefits to the fields of biomedicine, conservation of biodiversity, and aquaculture. The increasing need of using animal models for biomedical research has increased the demands for biobanking of animal materials for medical research. According to the Red List of the International Union for Conservation of Nature and Natural Resources, over 5,000 aquatic animal species worldwide are threatened, including fishes, molluscs, crustaceans, and corals. This has also lead to an increased interest in the creation cryobanks for conservation purposes. Global growth of intensive aquaculture has increased the needs for efficient and effective means, such as cryobanks, of conserving reproductive cells for greater flexibility in broodstock management, genetic improvement programs, and preservation of genetic diversity. Cryopreservation of many different cellular types has been studied, e.g., sperm, oocytes, embryos, somatic cells, and primordial germ cells or early spermatogonia. Cryobanks have been established for a range of aquatic species worldwide including Europe, USA, Brazil, Australia, and New Zealand. The management of these banks requires expertise and technical capacity in genetics, reproductive physiology, cryobiology, and data management. Cryopreservation protocols need to be carefully designed for each species and each type of cells to ensure optimal survival. The discussion will be focused on cryobanking in aquatic species including freshwater and marine fish species as well as invertebrates.

\section{O-26 Biobanking in a Challenging African Environment: An Update on Unique Experience from the SIREN and SIBS Genomics Projects}

\section{S. O. Diala}

College of Medicine, University of Ibadan, Ibadan, Oyo, Nigeria.

Background: Low-and-middle-income countries, especially in Africa, despite previous early commendable efforts, had been under-represented in the emerging discipline of biobanking. Support from the Human, Heredity, and Health in Africa (H3Africa) initiative has continued to bolster biorepository science and regional biobanks and has continued to increase awareness on biobanks across the continent.

Methods/Approach: The SIREN project, initiated in 2014 with support and funding from the H3Africa Initiative, is a transnational, multicenter, hospital- and community-based study of over 3,000 cases and 3,000 controls recruited from 16 sites in Ghana and Nigeria. Part of its objectives is to unravel the genetic and environmental factors that interact to produce the peculiar phenotypic and clinical characteristics of stroke among people of African ancestry whilst facilitating the development of new diagnostics, therapeutics, and preventative strategies. Subjects are first educated about unhealthy lifestyle practices that can predispose one to stroke and good lifestyle practices that can help to prevent stroke. Samples are then collected from the study subjects after they have given their consent, processed, and stored in $-20^{\circ} \mathrm{C}$ in the peripherals sites 
prior to shipment to SIREN central biorepository $\left(-86^{\circ} \mathrm{C}\right.$, freezers) in Ibadan every three months. Visual grading system to check hemolysis in the samples and a daily freezer temperature chart are some of the quality control measure we engage.

Results: The SIREN Biobank currently has 3,015 brain images, 92,950 blood fractions (serum, plasma, red blood cells, and buffy coat) accrued from 8,450 recruited subjects, and quantified and aliquoted good-quality DNA extracts from 6,150 study subjects. Three staff of the study were trained by the Institute of Human Virology, Abuja, in August 2018 on DNA extraction and quantification, samples management, and freezer work. One of the challenges is evacuation of samples from peripheral sites when prolonged power outage is experienced while the solution is tested and finding a trusted courier company to assist with immediate sample evacuation from peripheral site when the need arises.

Conclusion: This represents an invaluable resource for future research with expanding genomic and trans-omic technologies. This will facilitate the involvement of indigenous African samples in cutting-edge stroke genomics and transomics research.

\section{ITO-01 A Comparison between Automated Storage Refrigeration and Manual ULT Technology Identifying Improvements in Temperature Uniformity to Protect Sample Integrity}

C. Wolfenden

Brooks Life Sciences, Manchester, Lancashire, United Kingdom

Statement of the Problem: Biobanks typically cryopreserve biospecimens to safeguard their biochemical composition. Exposing samples repeatedly to temperatures above $-80^{\circ} \mathrm{C}$ for prolonged periods of time can degrade sample integrity. Sample storage technology usually involves temperature recovery following a door opening. Prolonged periods of temperature elevation due to lengthy recovery periods can affect your samples' viability. Surprisingly, it is quite common for upright ultra-low temperature (ULT) freezers to have temperature variances of up to $30^{\circ} \mathrm{C}$ between the top and bottom. Samples should be stored at uniform ULT temperatures to maintain viability.

Proposed Solution: A comparison study between manual upright ULT $-80^{\circ} \mathrm{C}$ freezers using both compressor-based technology and Stirling cooling system was compared with ULT chest freezer technology and the MaxCool Refrigeration in an automated sample storage system. The technology used in an automated store has many benefits over Typical 2-Stage Refrigeration as the MaxCool Cryochiller washes samples with cold air and can achieve $-150^{\circ} \mathrm{C}$ without going below 1 bar compared with a 2-compressor system achieving $-85^{\circ} \mathrm{C}$ but only with pressure below atmosphere. The temperature recovery is also superior due to exposing less than $1 \%$ of the storage environment for less than eight seconds per tray pick reducing the time needed for recovery back to operating temperature.

Conclusions: MaxCool Refrigeration in the automated store consists of three independent channels $2 \times$ MaxCool and $1 \mathrm{x}$ Liquid Nitrogen. It employs a non-flammable, non-toxic, HCFC-free refrigerant mixture in an auto-refrigerating cycle cooling process to provide operating temperatures from $-80^{\circ} \mathrm{C}$ to $-150^{\circ} \mathrm{C}$.

This results in a high level of sample temperature stability (less than $\pm 1{ }^{\circ} \mathrm{C}$ around the temperature set point of the Storage Compartment) and air temperature uniformity (less than $\pm 3^{\circ} \mathrm{C}$ around the temperature set point of the storage compartment) and is more consistent as less than $1 \%$ of the surface area of the tile wall opens at any time to remove samples from the $-80^{\circ} \mathrm{C}$ storage environment.

This process, which is thermodynamically more efficient than a multi-stage cascade process, combines all its refrigerants into a single multi-component stream circulated by one compressor. This system when compared with manual ULT freezer technologies offers greater sample and environment temperature stability.

\section{ITO-02 Digital Whole Slide Tissue Images Enriched by Morphometrics Provide Region of Interest (ROI) for Selected Technical Procedures}

A. Parwani ${ }^{1}$, R. Mandt ${ }^{1}$, D. G. Nohle ${ }^{1}$, D. A. Kellough ${ }^{1,2}$, L. W. Ayers ${ }^{1}$

${ }^{1}$ The Ohio State University, Columbus, Ohio, United States, ${ }^{2}$ Inspirata Inc., Tampa, Florida, United States

Background: The Cooperative Human Tissue Network (CHTN) is a NCI-sponsored prospective human tissue procurement program that provides human tissue, tissue derivatives, and other biospecimens to approved researchers. Selected investigators' research can be enriched by paraffin blocks with whole slide images (WSI) and/or WSIs with morphometric segmentation. Enriched WSI can be used as a guide in the selection of a region of interest (ROI) for tissue harvesting. Examples include tissue cores for construction of tissue microarrays (TMA), selecting tissue in macro and microdissection, tissue for DNA assays, or to select a ROI in a WSI for development of artificial intelligence (AI) algorithms.

Design: Tissue procurement procures a quality control (QC) sample tissue block reflective of the consented surgical remnant tissues at each procurement for an approved investigator. Each QC sample is formalin-fixed paraffin-embedded. A tissue section is H\&E stained and digitized (ScanScope XT, Leica Biosystems) to produce a WSI and morphometric segmentation (Tissue Studio, Definiens). The QC pathologist uses the Research Tissue Procurement - Information System (RTP-IS) to view the WSI and pathology report. They enter \% of ROI and \% necrosis by estimation or morphometric segmentation and select pertinent data from dropdown menus including final histopathological diagnosis. Researcher-directed technologists use the WSI or morphometric segmentation of that image to identify the ROI for the requested tissue sampling. The research tissue QC digital images, pathology report, and sample descriptive data provided associate with no PHI or donor identifiers and are maintained indefinitely. WSI, pathology reports, and data can be disbursed to qualified investigators with or without paraffin blocks as requested.

Results: One thousand ninety-seven paired H\&E image (.svs) and morphometric segmented image analysis result (.jpg) files for QC research tissue specimens from 2013-2016 are filed in RTP-IS. ROI area and \% from morphometrics were comparable to pathologist review ROI estimations for $90 \%$ of cases.

Conclusion: Research technical products such as TMA, microdissection, DNA assay, or diagnostic AI algorithm development can proceed using WSI with or without morphometric segmentation. The investigator may request and receive the paraffin block along with WSI and clinical data. For AI algorithm development, the investigator may receive only a copy of electronic data. Original electronic data remains in RTP-IS. 
ITO-03 A Simple, Rapid, Multiplex, Isothermal Amplification Assay (AmpFire) for Detection and Genotyping of Oropharyngealin Cancer HPV in Formalin-Fixed Paraffin-Embedded Tissues

Y. Wang

Atila BioSystems, Inc, Mountain View, California, United States

Rapid and accurate detection and identification of human papillomavirus (HPV) is important for both clinical management and population screening. Detection of HPV DNA from formalin-fixed, paraffin-embedded (FFPE) specimens has been a challenge as it usually requires lengthy and inefficient sample process. The AmpFire HPV assay (Atila Biosystems Inc.) incorporates a multiplex isothermal amplification to detect and genotype 15 high-risk (HR) HPV genotypes directly from raw samples without needing to extract or purify the DNA. The whole detection process requires a couple pipetting steps and can be completed within 2.5 hours. We performed analytic validation of Atila AmpFire Multiplex HPV assays on FFPE cervix/vulva and oropharynx diagnostic tissue samples. Limits of detection determined by plasmids cloned with HPV genotypespecific sequences were two copies/reaction for HPV16, HPV18, and some HR HPV genotypes, and 20 copies/reaction for the remaining HR HPV genotypes. The performance of the AmpFire assays in clinical samples was evaluated using 214 FFPE specimens. The AmpFire assay failed in one clinical specimen for an invalid rate of $0.5 \%$. The AmpFire assay detected HPV in clinical samples with positive percent agreements of $100.0 \%$ for HPV 16, $100.0 \%$ for HPV18, and $94.7 \%$ for non-16/18 HR$\mathrm{HPV}$, and $100 \%$ negative percent agreements for HPV16, HPV18, and non-16/18 HR-HPV. Qualitative detection agreement was obtained in the reproducibility study. In summary, the Atila AmpFire HPV assay demonstrated excellent analytic sensitivity and specificity for detection and genotyping of 15 HR HPV genotypes. Assay parameters of simple specimen processing, small sample size requirement, rapid turnaround time, and being near instrument-free render it well suited for HPV detection and genotyping in FFPE specimens.

\section{ITO-04 Integration of Virtual Slides in Biobanking}

C. Stephan, D. Spiegel

\section{KAIROS GmbH, Bochum, Germany}

KAIROS is an information technology (IT) company specializing in software for clinical research, biorepositories, and clinical trial management, which has been working with software solutions in the healthcare sector for more than 10 years. The focus lies in the development of interoperable, comprehensive information and documentation systems, following the FAIR principles.

The digitization of tissue samples opens up the world of digital image analysis, giving computerized systems a clear edge in pattern recognition over human capabilities. Also, nowadays there is no preview of sample material available, before samples are pulled from a biobank, making sample selection an additional challenge for the researchers. For the complete implementation of the advantages resulting from virtual slides, the step towards routine visual digital acquisition of biomaterial samples is indispensable.

Since high-resolution scans go hand-in-hand with the collection of large amounts of data, a smart IT solution is needed to support these data volumes with routine recording, classification, and evaluation. Recording the outcome of image analysis into structured information enriches the value of biobanks and allow for better sample cohort definitions.

Here, however, it is problematic that missing interfaces often prevent subsequent, progressive material characterization. At the same time, relevant references in the images are not recorded by information technology and recorded according to need, so that they remain inaccessible for the execution of scientific studies. The challenge here is not only the digitization of the specimens but the optimization of data structures and provision of appropriate tools to enable efficient transmission and presentation of the image data. With its CentraXX software solution, the KAIROS GmbH offers a more than suitable instrument for overcoming these hurdles. With the help of the software, both the sectional image and integrated annotations, as well as descriptions, can be viewed server-mediated. In this way, the virtual slide can be displayed as a preview in the system or via a virtual microscope via zooming functions.

The comprehensive digitization of tissue samples and the subsequent step of virtual microscopy requires more than just the samples themselves: What is needed is an IT solution that supports and promotes the future-oriented, digital steps of biobanks.

\section{ITO-05 Continuous Validation: A Lower Cost Alternative Means of Compliance}

R. Kriss

\section{KLATU Networks, Inc., Poulsbo, Wisconsin, United States}

In cold-storage and biorepository applications such as refrigerators, ultra-low temperature (ULT or -80s), and liquid nitrogen storage tanks where temperature-sensitive biological materials are stored, it is necessary to maintain continuously stable temperatures at multiple points inside the storage chamber.

The storage conditions are managed by internal policies or regulated by external standards. Temperature mapping and validation testing must be conducted at periodic intervals, such as at every six months, one year, two years, etc., on each coldstorage system to assess and document the uniformity and stability of temperatures in comparison to published accreditation standards. The testing is expensive because it is labor intensive and requires expensive test instrumentation or outsourced calibration services. The failure to have or maintain a validated system can result in loss of accreditation status, but it could also result in the loss of high-value research if temperature anomalies are not detected immediately when they occur.

A presentation by KLATU Networks will introduce a new monitoring technique called "Continuous Validation," enabled by new IoT sensor and statistical modeling architectures which eliminates most labor and expensive test instrumentation normally associated with traditional validation processes. As a result, the costs of maintaining compliance with validation standards can be reduced by $90 \%$.

\section{ITO-06 A Novel Deep Learning and Machine Vision System for Automating Blood Fractionation on a High-Throughput Liquid Handling Platform}

J. Atwood

PerkinElmer, Hopkinton, Massachusetts, United States

There has been an exponential increase in interest around plasma-derived circulating-free DNA (cfDNA) as a potential 
disease biomarker and the use of buffy coat as a source of bloodbased genomic DNA (gDNA) has experienced rapid adoption due to the attractive economics in biobanking environments.

Blood-based cfDNA and cfDNA/gDNA biobanking workflows start with centrifugation to fractionate whole blood into plasma, buffy coat, and erythrocyte layers. Highly automated environments require accurate determination of interface positions and volumes of the plasma, buffy coat, and erythrocytes layers, following blood centrifugation. This is especially critical for cfDNA and gDNA extraction as maximizing recovery, while limiting contamination, is imperative.

Here, we present a fully automated liquid-handling platform for blood fractionation. The core of the platform is a multiwavelengths color vision system that captures images of centrifuged blood samples. After pre-processing with traditional machine vision tools, the images are passed to a deep learning workspace that has been trained on a curated image dataset of human blood tubes from multiple samples, produced under variable lighting conditions. The deep learning neural networks classify which tube type is present, locate the blood tube in field of view, determine the tube top for mechanical reference, and then analyze the sample to calculate the volume of plasma and buffy that are present in the sample. Layer height and volume data are used to drive precise pipetting with a combination of conductive liquid level sensing and multi-point aspiration to accurately aspirate plasma and buffy coat for cfDNA and gDNA downstream purification.

To evaluate the deep learning approach, we compare layer height and volume measurements using progressively larger image training sets of human blood tubes relative to the traditional machine image analysis. As part of this study, height and volume accuracy is quantified as a function of training set size, sample variability, blood tube geometry, and environmental conditions to demonstrate the robustness of our deep learning algorithms. We further demonstrate that the approach produces high concentration and high quality cfDNA and gDNA extractions from plasma and buffy coat samples to offer a full walkaway liquid handling platform for blood fractionation. For research use only. Not for use in diagnostic procedures.

\section{ITO-07 Conditionally Reprogrammed Cells as a Living Biobank for Predictive and Precision Medicine}

X. Liu

Department of Pathology, Georgetown University, Washington, District of Columbia, United States

To date, it has not been possible to expand and indefinitely propagate cells derived from adult tissues while retaining lineage-commitment, normal growth control, and differentiation potential. Indeed, many primary cells (e.g., prostate, liver, lung, and pancreas) are incapable of being passaged for significant periods in vitro. We now describe a general method that rapidly expands both normal and malignant epithelial cells from diverse anatomic sites and mammalian species and does not require transfection with exogenous viral or cellular genes. Establishment of cell cultures from both normal and tumor tissue is highly efficient. Perhaps most important, cell cultures can be generated readily from core biopsies as well as cryopreserved human specimens. The robust nature of the technique is exemplified by the ability to produce $2 \times 106$ cells in five days from a core biopsy of breast tumor. Normal breast and prostate cultures retain a normal karyotype and differentiation potential and cell lines derived from tumors retain their tumorigenic phenotype. We will also describe several approaches that allow to enrich cancer cells from urine (for bladder cancer), blood (for prostate cancer), and pleural effusion (for non-small cell lung carcinoma). These cancer cells from liquid biopsies were cultured and further propagated in suspension and/or adherent cultures using a specialized cell culture medium. We also confirmed genetic alterations in both original tumors and cancer cells from liquid biopsies, for example, mutant EGFR for lung cancer, mutations of hTERT promoter for bladder cancer. We also reported that these cancer cells from liquid biopsies were used for identify therapies for the patients. For example, a woman patient with metastatic NSCLC harboring mutant EGFR was primarily resistant to TKI treatment; we were able to identify a combination of chemotherapy for the patient. The ability to produce inexhaustible cell populations as a living biobank from small biopsies and cryopreserved specimens has the potential to transform biobanking repositories and current pathology practice by enabling genetic, biochemical, metabolomic, proteomic, and biological assays, including chemosensitivity testing as a functional diagnostics tool for precision cancer medicine.

\section{ITO-08 Ten Years of the Cohort \& Biobank: A Bibliometric Analysis via CiteSpace}

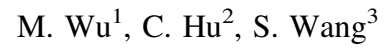

${ }^{1}$ MOE-Shanghai Key Laboratory of Children's Environmental Health, Xinhua Hospital affiliated to Shanghai Jiao Tong University School of Medicine, Shanghai, China, ${ }^{2}$ School of Public Health, Shanghai Jiao Tong University School of Medicine, Shanghai, China, ${ }^{3}$ School of Public Health, Shanghai Jiao Tong University School of Medicine, Shanghai, China

Background: Biobank and cohort are the most popular words these days in the global scientific research field. Cohorts with biological material are necessary to investigate the possible biological mechanisms behind the disease, which have drawn an increasing amount of attention over the past decades. The objective of this study was to evaluate the origin, current trend, and research hotspots on cohort and biobank.

Methods: After filtering out a small number of duplicate records, we obtained 932 original records with 28,542 references, published between 2009 and 2018 from the Science Citation Index Expanded (SCI-E). The CiteSpace 5.5.R2 was used to perform the cooperation network analysis, key words co-occurrence and burst detection analysis, and reference co-citation analysis.

Results: The number of publications on cohort and biobank was increasing over the past decade. Tai Hing Lam from Department of Community Medicine, University of Hong Kong, was found to be the most productive researcher in this field. The percentage of publications in England (38.30\%) was the highest all over the world. Risk, biobank, meta-analysis, cohort, disease, risk factor, association, mortality, population, and health were the top 10 high-frequency keywords in co-occurrence analysis. Metabolic syndrome was the most strength burst key word in this field, followed by Hong Kong, Guangzhou biobank cohort, and personalized medicine. Moreover, of all the references for 932 articles included in the study, the article titled "UK biobank: an open access resource for identifying the causes of a wide range of complex diseases of middle and old age" published in PLoS Med by Sudlow C et al. in 2015 was the most frequently co-citated reference in this field. The largest cluster was labeled as Guangzhou biobank cohort study.

Conclusions: This study provides an insight into cohort and biobank and the valuable information for biobankers to identify new perspectives on potential collaborators and cooperative countries/regions. 
ITO-09 Setup of a Digital Biobank of Chest X-Ray Images for the Independent Evaluation of ComputerAided Solutions for Pulmonary Tuberculosis

S. Ongarello ${ }^{1}$, S. Kik ${ }^{2}$, C. Denkinger ${ }^{2}$, S. Gelaw ${ }^{3}$, M. Ruhwald ${ }^{2}$

${ }^{1}$ Data Services and Biobanking, FIND, Geneva, Switzerland,

${ }^{2}$ FIND, Geneva, Switzerland, ${ }^{3}$ IOM, Manila, Philippines

Statement of The Problem: Chest X-ray (CXR) has long been part of the diagnostic approach for pulmonary tuberculosis (TB), but inter-reader variability among human interpreters is considerable and access to trained readers is limited, particularly in high TB burden countries. Computer-aided detection (CAD) technology can analyse a digital CXR image and produce an interpretation that estimates the likelihood that the image is from an individual with active TB. Demonstrated evidence on the accuracy of CADs is limited, heterogeneous, and performed in low-quality studies.

Proposed solution: FIND (www.finddx.org) and the International Organization for Migration (IOM) established a representative archive of CXR images with related demographic and clinical data, including a representative spectrum of TB-associated lung pathology and pathology of differential diagnoses distributed according to gender, HIV-status, age, and geographical origin. This archive will be used to assess the accuracy of CAD systems for identifying pulmonary abnormalities consistent with $\mathrm{TB}$, compared against three different reference standards: 1) human readers, 2) microbiological results, and 3) a composite score based on microbiology and human interpretation for two use-cases for CAD (active screening of individuals who are not seeking care, and triaging of individuals seeking care because of symptoms suggestive of TB). Images and data for over 5,000 individual patients have been collected from 10 collaborating institutions in seven countries. The data are currently stored in a dedicated server at FIND to prevent online distribution of the files, which can only be accessed in an offline-mode for the purpose of testing each CAD system on images that were never used in their AI-based training process. FIND is responsible for the harmonization of the data across contributors, in addition to the final analysis of the performance of each CAD system.

Conclusions: Sensitivity, specificity, and diagnostic yields will be estimated on the same set of images for each CAD system, and alternative thresholds will be recommended to manufacturers if necessary.

The results will be presented at the WHO Guideline Development Group meeting in 2020 to inform the guideline development process on the use of CXR and CADs for the diagnosis of pulmonary TB. 


\section{POSTER ABSTRACTS}

\section{Biobank Tools}

\section{PA-01 Effect of Educational Intervention on Knowledge and Attitude towards Biobanks among Medical Team in Egypt}

S. W. El Khadry ${ }^{1}$, A. Abdallah ${ }^{1}$, M. Youssef $^{1}$, H. Abdeldayem $^{2}$, L. Dorgham ${ }^{1}$, S. Ezzat ${ }^{1}$

${ }^{1}$ Epidemiology and Preventive Medicine Department, National Liver Institute, Menoufia University, Egypt, Shebin Elkom, Menoufia Governerate, Egypt, ${ }^{2}$ Hepatobilary Surgery Department, National Liver Institute, Menoufia University, Egypt, Shebin Elkom, Menoufia Governerate, Egypt

Background: Biobanks have become increasingly important for the study of health and disease. In the past few years, few biobanks have been established in Egypt. However, there is limited knowledge about biobanking among Egyptians. The objective of this work is to assess the knowledge, attitude, and opinions of a medical team towards biobanking issues, and to implement an intervention educational program to improve their knowledge and attitude.

Participants \& Methods: This study was a quasi-experiment which was conducted at National Liver Institute (NLI), Menoufia University, Egypt, where a sample selected with an intervention educational study on 127 participants of a medical team (Master or MD students, demonstrators or residents, assistant lecturers, lecturers, and pharmacists) who had been: 1- pre-assessment on knowledge, attitude towards biobanking through adopted questionnaire (BANKS), 2- education intervention on biobanking and related issues, and 3- post-assessment (reassessment by BANKS questionnaire), which was adopted from (Wells et al., 2014). Approximately 15 meetings and scientific days at different departments were done to reach more doctors and young researchers working at NLI.

Results: Pre-intervention, there were significant differences between participants' jobs and their knowledge where $17.6 \%$ of pharmacists and $9.1 \%$ of assistant lecturers were the only participants who had good sufficient knowledge; others had insufficient knowledge $(p$-value $=0.02)$. There was no significance difference between participants' jobs and their attitude pre-intervention. Fortunately, the education intervention study was effective in increasing percent of good knowledge groups from nearly $4.2 \%$ pre-intervention to nearly $78.3 \%$ post-intervention and this effect was statistically high significant ( $\mathrm{p}$-value $<0.001$ ). The education intervention study was effective in increasing percent of positive attitude from $25 \%$ pre-intervention to $79.2 \%$ of medical team participants post-intervention and this effect was high statistically significant (p-value $<0.001$ ).

Conclusion: Despite limited knowledge and moderate attitude of the NLI medical team about biobanks and biobanking, the educational intervention study had significant increasing knowledge and attitude for all studied medical teams. Therefore, the implementation of an intervention educational program and workshops are highly recommended for medical teams working in NLI and other hospitals.

\section{PA-02 Rationale, Content, and Reported Use of the National Cancer Institute's Biospecimen Research Database}

K. B. Engel ${ }^{1}$, E. Casas-Silva ${ }^{2}$, L. Campbell ${ }^{3}$, S. Greytak ${ }^{3}$, P. Guan' ${ }^{2}$, H. Moore 2

${ }^{1}$ Preferred Scientific Group, Merritt Island, Florida, United States, ${ }^{2}$ BBRB, National Cancer Institute, Bethesda, Maryland, United States, ${ }^{3}$ Kelly Government Solutions, Bethesda, Maryland, United States

Statement of Problem: Variability in human biospecimen collection, preservation, processing, and storage practices can adversely affect downstream results, subvert study conclusions, and complicate meta-analysis efforts. Despite recognition of Biospecimen Science as a field of scientific study by the biobanking community, relevant literature remains dispersed across hundreds of journals and is, therefore, challenging to locate with standard literature search engines, such as PubMed. In addition, information about the standard operating procedures (SOPs) utilized by different institutions can be lacking, hindering harmonization of biospecimen handling practices and further contributing to preanalytical variability.

Proposed Solution: The National Cancer Institute's Biorepositories and Biospecimen Research Branch developed the Biospecimen Research Database (BRD; http://biospecimens.cancer.gov/ brd) to serve as a centralized literature repository of peer-reviewed articles specific to the field of human Biospecimen Science, and to house a collaborative library of SOPs from a diverse set of biobanks. The aims of the BRD are to improve the quality of biospecimens used in discovery and clinical research by providing researchers and biobanks with information about preanalytical variability and to facilitate harmonization of biobanking practices by supplying a forum to share SOPs developed and used by established biobanks.

Conclusions: Since its inception in 2008, the BRD has grown to include more than 2,800 curated articles published in 700 different scientific journals and over 500 SOPs contributed by more than 70 source organizations including the U.S. government and international, private, and non-profit biobanks. The BRD has proven to be a resource for the biobanking and scientific community with an average of 1,200 unique visitors and 20,000 SOPs downloaded per month. Based on publications by authors not affiliated with NCI, the BRD has been used as a literature database, a vehicle to publicly share and cite specific protocols, and to survey biobanking SOPs to identify common practices. We invite you to explore the $\mathrm{BRD}$ as a resource and to consider contributing relevant publications and SOPs addressing biospecimen handling to the database. To share your BRD experience with NCI, email us at ncibbrb@nih.gov.

\section{PA-03 Self-Developed Human Biobank Information System (HuBIS) to Support Multiple Biobanking Processes}

D. Kim, S. Lee, M. Chu, E. Kim, J. Jeon

Division of Biobank for Health Science, Korea National Institute of Health, Cheongju-si, Korea 
The National Biobank of Korea (NBK) has operated the selfdeveloped Human Biobank Information System (HuBIS) which allows to efficiently manage various informations about the storage and distribution of biosamples. The HuBIS can manage biosample-related inventory data of both a central biobank (NBK) and 17 regional biobanks which comprise the Korea Biobank Network. HuBIS consists of three distinguished systems, HuBIS_Track, HuBIS_Sam, and HuBIS_Desk. First, HuBIS_Track is a system to support the barcode labelling and management for cross-talk between cohort sites and the biobank, currently installed in at least 100 cohort sites across the country. Second, HuBIS_Sam is a management system for the collection, storage, and release of human bioresources, currently installed in 56 out of 72 legally approved human biobanks in Korea. HuBIS_Track and HuBIS_Sam enable biobankers or administrators to manage biobanking processes efficiently. Third, HuBIS_Desk has been developed as the one-stop distribution platform with the following key functions: to search for available bioresources, to support online utilization consultation, to submit access requests and documents, and to report bioresource utilization results. The NBK has conducted maintenance projects to improve the utility and functions of HuBIS, such as annually performance improvement, operation of Helpdesk, and regular education programs for unskilled users. In the future, HuBIS will be improved to be suitable for automated biobanking processes and highly compatible for international standards (e.g., HL7, ISO20387, ISBER Best Practices).

\section{PA-04 The Impact of DNA Extraction Methods on Quantification for Normalization}

E. A. Feil ${ }^{1}$, A. Bilyeu ${ }^{1}$, V. Wagner ${ }^{1}$, R. Busby ${ }^{1}$, J. Meyer ${ }^{1}$, M. Cicek ${ }^{1,2}$

${ }^{1}$ Biospecimens Accessioning and Processing, Mayo Clinic, Rochester, Minnesota, United States, ${ }^{2}$ Department of Laboratory Medicine and Pathology, Mayo Clinic, Rochester, Minnesota, United States

Background: DNA quantification data accuracy is critical in sample normalization for downstream analytical assays. Biorepositories often have a variety of extraction methods available to them but a singular method to quantify. Inaccuracies in quantification data can lead to problems such as assay failures for researchers downstream. Our laboratory has developed a protocol to normalize all samples extracted to a set concentration based off of data received from a spectrophotometer.

Methods: DNA was extracted from human whole blood samples via two different methods; 4-mL blood, fresh and frozen, was split into two sub groups and DNA was extracted by either automated precipitation or automated bead-based extraction method. Following extraction samples were then quantified using a spectrophotometer as well as a fluorescent dye.

Results: Recently our biorepository laboratory has discovered that change of platform for spectrophotometric quantification impacted the difference observed between dsDNA and total DNA concentrations based on the extraction method.

Conclusion: The quantification algorithms provided by the vendors are proprietary and do not always give accurate and consistent results. We determined that we needed an algorithm that considers extraction method as well as blanking system in order to present accurate quantification data.

\section{Biobanking Profiles}

\section{PB-01 Biobank Profile: China National GeneBank}

\author{
Y. Shao, J. Chen, P. Qian
}

China National GeneBank, Shenzhen, China

As the first integrated national gene bank in China, CNGB is committed to supporting scientific research, public welfare, innovation, and industrial infrastructure construction. Based on the abilities to "read, write, and store" genetic information, CNGB serves as an open platform that provides access to and enables exchange and sharing of genetic data and resources to advance the development of the life sciences and bio-economy.

China National GeneBank (CNGB) is a non-profit organization supported by the Chinese Government. CNGB has built an integrated infrastructure of "Three Banks and Two Platforms." "Three Banks" represent the Biorepository, Bio-informatics Data Center, and Biological Resource Center of Plants, Animals and Micro-organisms. "Two Platforms" include Digitalization Platform and Synthesis and Editing Platform.

Reading: An automated, informatized platform with petabytes throughput each year, the Digitalization Platform is dedicated to supporting research projects in precision medicine, agricultural breeding, marine development, and biodiversity conservation.

Writing: CNGB is building a world-leading writing platform that is capable of synthesizing 10 million base pairs per year. The platform enhances application of synthetic biology and gene editing technologies in natural product biosynthesis, disease diagnosis and treatment, modern agriculture, environment protection, and other areas.

Storing: China National GeneBank DataBase (CNGBdb) is an integrated platform built for biological data sharing and application. Based on big data and cloud computing technologies, it provides data services such as archive, analysis, search, data management, and scientific databases to researchers around the world. E-Biobank (EBB) bridges the gap between biosample holders and users by aggregating bio-sample and biobank information at home and abroad in a standardized method. It aims to create an equal and open environment for sample sharing and enhance the reasonable utilization of bio-samples.

Public Service Platform: Opening up its scientific infrastructure to the public, CNGB provides public services based on its powerful abilities to "read, write, and store" genetic information to support the development of life science research and industry: experiment platform leasing, bio-technology services, DNA synthesis, and biobank construction consultation.

\section{PB-02 Biomarker Discovery: Role of Dasman Diabetes Biobank, State of Kuwait}

D. Sriraman ${ }^{1}$, V. Atizado ${ }^{1}$, A. Mathur ${ }^{1}$, B. S. Chandy ${ }^{1}$, R. M. Jose ${ }^{1}$, V. Vijayan ${ }^{1}$, M. Melhem ${ }^{3}$, M. Abufarha ${ }^{1}$, F. Al Mulla ${ }^{2}$

${ }^{1}$ Special Services Facilities, Dasman Diabetes Biobank, Dasman, Kuwait, ${ }^{2}$ Dasman Diabetes Institute, Dasman, Kuwait, ${ }^{3}$ Special Services Facility Omics Core, Dasman Diabetes Institute, Dasman, Kuwait

Background: Biomarkers are defined as cellular, biochemical, or molecular alterations that are measurable in biological media such as human tissues, cells, or fluids. Biomarker dis- 
covery involves series of process that is identification, verification, and validation. These are possible only on the availability of high quality of specimens. Dasman Diabetes Biobank (DDB) is a core facility established by Dasman Diabetes Institute (DDI) funded by Kuwait Foundation for Advancement of Sciences in 2009 to accelerate the research and personalized treatment for diabetes and its complications. DDB collects, processes, stores, and distributes biospecimens and associated data for use in future research. DDB is providing high-quality biospecimens and comprehensive and reliable data and involving in many research projects which results would be highly useful in diabetes research, biomarker discovery, and translational research.

Methods: DDB has collaborations with Ministry of Health and Kuwait University and various departments within DDI. It has international collaborations with Texas Diabetes Institute, Forsyth Institute, University of Cambridge, Harvard Medical School, and Public Health. DDB developed standard operating procedures (SOPs) for all its sample processing. It is mandatory that participants sign an informed consent which includes primarily samples agreed to use for specific projects and whether the left-over samples could be used for future research purposes.

Results: DDB collects, processes, and stores a wide array of samples (plasma, serum, nucleic acid, buffy coat, urine, saliva, and adipose and skeletal tissues). All the samples are processed using the validated SOPs. DDB associated with more than 70 projects for research and biomarker discovery related to diabetes and its complications. DDB procured samples from more than 35,000 participants.

Conclusions: It is the first of its kind biobank in the State of Kuwait and provides support to the research community. These samples have a direct impact on biomarker research. DDI's research division published more than 200 scientific articles which includes the recently identified hormone ANGPLT8 (betatrophin) that has been associated with two functionally important processes in the development of type 2 diabetes, insulin resistance, and lipid metabolism. We have been involved in many research projects whose results are highly productive on patient care and diabetes research, biomarker discovery, and translational research.

\section{PB-03 Presentation of the National Tumor Bank of the National Cancer Institute, Rio de Janeiro / Brazil: Theoretical and Practical Aspects}

\section{D. Accioly}

National Tumor Bank/Research Coordination, National Cancer Institute, Rio de Janeiro, Brazil

INCA, the National Cancer Institute, is a public cancer center linked to the Ministry of Health. The BNT, National Tumors Bank, is an organized collection of human biological material, tissues, blood, genetic material, and associated information in accordance with national and international norms and standards. Its purpose is to collect and store human biological material from care activities, according to pre-defined operational procedures, with the purpose of validating hypotheses about the processes that lead to the onset and evolution of neoplastic diseases, contributing to a better diagnosis and treatment and ensuring the quality, safety, and technical and ethical adequacy of stored material and associated information. Patient collection is performed by nurses at the three INCA Units, where the informed consent form is provided: Informed Consent for Assignment, Storage and Use of Biological Mate- rial. BNT-INCA was created in 2005 and is linked to INCA's Research Coordination. It is a valuable resource in cancer research because it facilitates the availability of quality tumor samples associated with pathological anatomical patient information. The collections are performed in the surgical centers of the three units, and also in the endoscopy and bronchoscopy outpatient clinics. We are a team of four nurses, three collection technicians (histotechnicians), two technologists (biologists), one lab technician (biologist), one operational, one administrative, one quality analyst, one IT analyst, and one general coordinator. We currently store over 160,000 samples, including tumors, normal tissue, blood, and derivatives, from nearly 46,000 donors. We follow a collection protocol in operating rooms and outpatient rooms. We routinely perform the blood processing of donor patients from the three institution units and provide all technical support for the extraction of genetic material from the samples to the researchers. In addition we have four internal projects underway: tumor bank certification, quality control of specimens stored for over six years $\times$ recent specimens of the same morphology, assessment of cold ischemia times of surgical specimens with quality analysis of the specimen, RNA, and validation of a prostate tumor collection protocol.

\section{PB-04 Taking Your Collection Off-Road: A Case Study on the Effects of Deviations from Protocol}

\author{
D. L. Ellisor ${ }^{1}$, J. Trevillian ${ }^{1}$, C. Davis ${ }^{1}$, T. Rowles ${ }^{2}$, R. Pugh ${ }^{1}$ \\ ${ }^{1}$ National Institute of Standards and Technology, Charleston, \\ South Carolina, United States, ${ }^{2}$ National Oceanic and \\ Atmospheric Administration, Silver Spring, Maryland, \\ United States
}

The National Institute of Standards and Technology (NIST) has been involved in establishing strict collection and storage protocols for biological and environmental samples for 40 years. These standardized protocols are necessary to maintain sample and data integrity within not only an individual sample, but also when comparing samples collected across multiple years. Departures from a protocol can occur in the field due to limited staff, limited supplies, difficult weather conditions, or other unforeseen circumstances. Such is the case in remote areas like the Alaskan coastline, where tissue samples are collected from deceased marine mammals as part of the National Oceanic and Atmospheric Administration's Alaska Marine Mammal Tissue Archival Project (AMMTAP). Though protocol deviations are always noted, it is critical to understand the effects they have on specific downstream analyses. Here we present a case study using marine mammal tissues collected as part of the National Marine Mammal Tissue Bank and archived at the NIST Biorepository located at the Hollings Marine Laboratory in Charleston, SC. Blubber, liver, and kidney samples were collected from four deceased bottlenose dolphins (Tursiops truncatus) that stranded along the southeast coast of the U.S. This species was chosen as a surrogate for Alaskan marine mammal species, from which protocol-adherent samples can be difficult to obtain. Each tissue was split into two samples; one was immediately processed and archived according to the standardized protocol while the other was processed using a common deviation protocol involving an additional freeze/thaw cycle prior to tissue processing and archival. After the final freezing in LN2 vapor-phase freezers $\left(-150^{\circ} \mathrm{C}\right)$, each tissue was cryohomogenized and divided into multiple fresh frozen aliquots. The aliquots were assessed for various project-specific endpoints to compare the standardized and deviated protocols. 


\section{PB-05 Establishment of Beijing Biobank of Clinical Resources (BBCR) for Mental Disorders}

M. Liu, G. Zhang, G. Wang

Beijing Anding Hospital, Capital Medical University, Beijing, China

Background: Beijing Anding Hospital, Capital Medical University in Beijing is a special hospital for mental disorders, The Beijing Biobank of Clinical Resource for Mental Disorders (BBCR-MD) was established at Anding Hospital in 2011.The BBCR was officially opened in January 2009, connecting over 20 hospitals located in Beijing. The work of BBCR is focused on management system, quality control and standard operating procedures (SOPs) of processing, storage, and distribution of clinical resource and biological material with human disease research.

Methods: Methods were in accordance with the third edition of Best Practices for Repositories: Collection, Storage, Retrieval and Distribution of Biological Materials for Research and certification documents of the International Organization for Standardization (ISO) to establish BBCR-MD, including quality control, informed consent, clinical resource, and specimen collection and preservation. Special standardized operational procedures for BBCR-MD were developed; we also collected clinical data and venous blood samples after signed informed consent. Specimens were stored at $-80^{\circ} \mathrm{C}$ in a refrigerator. The clinical data of BBCR-MD is only open for professionals of the Beijing Anding Hospital at present. Prior to export, the data are anonymized to ensure the security and privacy protection of the participants. To date, samples were only distributed to the researchers who stored them. In the future, clinical data and associated samples can be made available to researchers around the world. The BBCR-MD appointed an Approval Committee which reviews all applications received and grants permission to access the stored samples and data for research.

Results: Between June 2012 and October 2019, more than 9000 participants were enrolled in BBCR-MD; over 106000 tubes of blood derivatives were collected and stored. Specimens included plasma, serum, erythrocytes, leukocytes, lymphocytes, urine, feces, and DNA. Except mental disorders (major depressive disorder, bipolar disorder, schizophrenia, anxiety disorder, obsessive-compulsive disorder), high-risk population and health control were also taken into account.

Conclusion: The construction of BBCR-MD is a systematic work. Its functions will be increasing in exploring pathogenesis, developing technological innovation for early detection, novel therapeutic strategies, and individualized therapy.

PB-06 Update on Successful Biobanking in a Challenging Environment, Ibadan, Southwestern Nigeria: A Case Study

A. Odetunde ${ }^{2}$, A. Ademola ${ }^{6}$, F. Onakpoma ${ }^{1}$, E. Fatunla ${ }^{1}$, M. Ajani ${ }^{1}$, B. Okedere ${ }^{3}$, O. Akinyele ${ }^{4}$, A. Falusi ${ }^{2}$, F. Olopade ${ }^{5}$, T. Yoshimatsu ${ }^{5}$

${ }^{1}$ Department of Pathology, University College Hospital, Ibadan, Nigeria, ${ }^{2}$ Institute for Advanced Medical Research \& Training, College of Medicine, Ibadan, Nigeria ${ }^{3}$ Breast Cancer Laboratory, Institute for Advanced Medical Research \& Training, College of Medicine, Ibadan, Nigeria, ${ }^{4}$ Healthy Life All Foundation, House 38, Ibadan, Nigeria, ${ }^{5}$ Section of Haematology and Oncology, Chicago, Illinois, United States, ${ }^{6}$ Surgical Oncology Unit, Department of Surgery, University College Hospital, Ibadan, Nigeria
Objective: Breast cancer biobanking was established at the University of Ibadan College Hospital (UI/UCH) in 2009 to accelerate progress in gaining a better understanding the genetic basis of breast cancer in women from southwest Nigeria, and improve patient care through development of better diagnostic and prognostic markers.

Methods: Patients were enrolled after written informed consent had been obtained. Mastectomy and multiple biopsy specimens were harvested and immediately preserved in fixative medium. Formalin was used for paraffin block (FFPE) preparation, and PAXgene was used for genetic analyses. Collected materials were sent to the tissue banking officer at the pathology department for accession along with verified clinical information from the surgery clinic. FFPE tissues were banked after histopathological assessments were performed. Samples preserved in PAXgene were stored in $-80^{\circ} \mathrm{C}$.

Results: Three hundred and thirty-three patients were enrolled between March 2009 and July 2018. Genomic DNA were extracted from matched peripheral blood, with median concentration of $192.2 \mathrm{ng} / \mu \mathrm{l}(47.8-693.3 \mathrm{ng} / \mu \mathrm{l})$ and median A260/ 280 of 1.88 (1.53-2.01). Immunohistochemistry for estrogen receptor and progesterone receptor were performed on all samples, and reviewed by pathologists. RNA was extracted on nearly half of the samples for RNASeq in the U.S. with good quality.

Challenges: One of the challenges at the outset was the unavailability of uninterrupted power supply; however, we were able to surmount this by procurement of solar power inverter system which now gives 24-hr uninterrupted power supply. The current challenge is lack of a real-time inventory system which we hope to overcome in the near future as this will enable real-time, global access to our data for research purposes and international collaboration.

Conclusion: Tissue banking is essential to translational research. We successfully developed a robust infrastructure for tissue banking of malignant tissues for molecular analysis under IRB approved protocol. After implementing best practices, quality of banked samples has dramatically improved and our results with RNASeq and NeXt Gen sequencing will be presented and shared with other participants.

\section{PB-07 The Vital Function of Suzhou Biobank in Future Medical Research and Precision Medicine}

\section{Y. Zhan}

Suzhou Center for Disease Control and Prevention, Suzhou, Jiangsu, China

Suzhou Biobank, the successor of Suzhou Serum Bank, was approved to start construction as a practical project by the government in 2010. The design concept of the project stemmed from the Kadoorie Biobank Study in China (KBSC), a prospective health study investigating effects of lifestyles, physical and biochemical indicators, and environmental and genetic factors on major chronic disease.

As a central city of Yangtze River Delta and an important area of "the Belt and Road," Suzhou has a developed economy, convenient waterway transportation, dense population, and abundant sample resources. There are 7 million permanent residents in Suzhou, so the number and diversity of population provide abundant resources for Suzhou biobank to carry on sample collection and storage. The samples for storage mainly are whole blood and the present sample capacity is about 1,040,000 tubes.

We will integrate advanced service concept and management practice with social development, make use of modern 
genetic technology and biological information technology, and take advantage of abundant samples and life information, aiming to provide all-around health services for the people and to promote rapid development of precision medicine

\section{PB-08 Construction of Kawasaki Disease Cohort in the South of China}

L. $\mathrm{Pi}^{1}, \mathrm{X} \cdot \mathrm{Gu}^{1,2}, \mathrm{D} . \mathrm{Che}^{1}$

${ }^{1}$ Department of Clinical Biological Resource Bank, Guangzhou Women and Children's Medical Center, Guangzhou Medical University, Guangzhou, China, ${ }^{2}$ Department of Blood Transfusion, Guangzhou Women and Children's Medical Center, Guangzhou Medical University, Guangzhou, China

Background: Guangzhou Women and Children's Medical Center is the largest women and children's hospital in the south of China. The biobank established in 2015 is one of the largest biobanks in the south of China, and there are more than $1,200,000$ bio-samples that have been collected. Several severe diseases cohorts have been built to track the patients well and continually, and Kawasaki disease is one of these cohorts.

Methods: The recruitment of the Kawasaki disease cohort was started in 2016 and applies highly standardized procedures. Standard quality management system according to ISO 20387 was established to improve the quality of bio-samples. According to the character of Kawasaki disease, we have collected the clinical samples of multiple time points, such as acute stage, subacute stage, and recovery stage. The samples collected in the acute stage were divided into before and after gamma globulin treatment groups. Because the serious complication of Kawasaki disease is coronary artery lesions, and some of the patients will even develop coronary artery aneurysms, Kawasaki disease needs long-term follow-up. We set up a long time sample collection plan with a lot of time points, such as every month after onset to three months, every three months after onset to a year, and every six months in the following time. We collected multiple sample types for the aim of the study to gain information of the development of Kawasaki disease: ACD anticoagulant whole blood was used to gain PPP, PLT, PBMC, and granulocytes for various studies. Urine, stool, and serum at various time points were also collected. Under the case that pediatric samples are rare and precious, we have made huge effort to increase the collection of children's limited blood. Samples after clinical test are collected, such as the remaining samples after the test of blood count, which are qualified whole blood samples to extract DNA.

Results: The Kawasaki disease cohort has encompassed more than 2,000 participants with 43,000 samples.

The Kawasaki disease cohort plays a very important role in the research. More than 20 papers published in mainstream journals at home and abroad are based on the samples or samples information.

Conclusion: The experience of Kawasaki disease cohort is very valuable for the construction and management of chronic diseases.

\section{PB-09 Next Generation Biobanking - Managing Multiple Requirements in the Heidelberg Cardiobiobank (HCB)}

S. Sandke, T. Heimberger, T. Weis

Heidelberg CardioBiobank (HCB), University Hospital Heidelberg, Heidelberg, Germany
Background: In the era of precision and personalized medicine, clinicians and experimental researchers are focusing on analyzing genomics, proteomics, and metabolomics of human patients to identify promising therapeutic targets or novel biomarkers. To facilitate this intention, hospitals establish biobanks with collections of biosamples annotated with their clinical data. The Heidelberg CardioBiobank (HCB) operates as a hospital-integrated Biobank, mainly for the Department of Cardiology as well as a core Biobank for several national and international studies.

Method: To ensure highest quality of biosamples, which is mandatory for cutting-edge translational research and precision medicine, we evaluated and optimized entire workflows for sample collection, logistics, and storage. In addition different procedures of sample processing were tested. Recording biosamples results in large datasets, which requires an IT-system enabling efficient and secure handling of data information. We therefore aimed for a customized solution, fitting our requirements.

Results: First of all we integrated new applicable and innovative technologies, depending on sample type or study requirements. To reach highest and consistent standards we convert manual sample processing in automated systems. Further standard operating procedures were developed for all preanalytical processes, starting with the collection of biomaterial until their final storage. This resulted in a significant improvement of sample quantity and quality. For data collection, we established an individualized IT solution using a customized research portal for biobanking and clinical studies named CentraXX (KAIROS GmbH).

Conclusions: The introduction of standardized and almost fully automated sample processing systems into the HCB produces the highest quality and integrity of biomaterials which is essential for translational research. The customized CentraXX system facilitates functional data modeling and enables an efficient search between clinical-, study-, and sample-related data. Embedding new technologies into the workflows allows the $\mathrm{HCB}$ to be prepared for the future and operates for new and divers clinical studies.

\section{PB-10 Challenges of Establishing a Biobank in Upper Egypt}

R. Mohamed ${ }^{1}$, F. Ghaleb ${ }^{2}$, N. K. Kordy ${ }^{1}$, S. Ezzat ${ }^{1,3}$

${ }^{1}$ Research, Shifaa Al-Orman Hospital, Luxor, Egypt, ${ }^{2}$ Shifaa Al-Orman Hospital, Luxor, Egypt, ${ }^{3}$ National Liver Institute, Shibin, Egypt

Background: The Shifaa Al Orman Oncology Hospital (SOH) was established in 2016 aiming to treat cancer patients for free in Upper Egypt. The hospital vision was not only to provide quality patient care but also to conduct translational and clinical research. The SOH-biobank (SOHB) was established in 2017 as an integral part of the research infrastructure. Our aims are to establish an ISBER-compliant biobank, and to highlight the challenges of the SOHB implementation and how we overcame these challenges.

Methods: All SOHB research and biospecimen collection from patients is performed in accordance with an SOH-IRB approved protocol. Standard operating procedures (SOPs) were written with strict adherence by the Research department team. Detailed job description was written for each individual with KPIs to evaluate their performance. Blood samples are collected after participant recruitment occurs in the biobank program through obtaining a consent form. Only the newly diagnosed patients are recruited. After blood collection, the blood is 
processed and is stored in $-80^{\circ} \mathrm{C}$ freezers. Tissue samples are kept in liquid nitrogen. A database is developed to store and document sample information. Sample deviations are recorded according to the SOPs to ensure the sample quality.

Results: Until September 2019, the SOHB has recruited 2150 newly diagnosed cancer patients. We have 14,400 samples. We faced many challenges during the implementation; the first was the unavailability of budget to buy commercial biobank software. Therefore, we have build our own software that integrated with the hospital system including all the preanalytical and storage data present in the specialized software. The second challenge was lack of awareness across the physicians; thus, we implemented orientation lectures. Physician compliance in terms of percentages increased from $27 \%$ and $33 \%$ in two random months before orientation to $60 \%$ and $66 \%$ in two random months after orientation. The third challenge was initiating biobank workflow within the patient workflow not to burden the hospital system and/or the patient with extra procedures. Consent is administered to the patient at the laboratory wait area. If the patient agrees to participate, the blood is drawn during phlebotomy for ordinary medical care.

Conclusion: Although establishment of biobank in an Upper Egypt Hospital is challenging, it is doable with the availability of intention and qualified personnel.

\section{PB-11 Development of a Regional Sustainable Disease-based Biobank to Improve Biospecimen Utilization and Research Productivity}

\section{Zhang, L. Zhang, H. Zhang}

\section{Beijing Biobank of Clinical Resources, Beijing, China}

Background: Beijing Biobank of Clinical Resources (BBCR) is one of the key initiatives sponsored by Beijing Municipal Science \& Technology Commission (BMSTC). The resources are the collaboration and partnership with one central institution (Capital Medical University) and fourteen specific hospital-based satellite biobanks. With a total of $\$ 50$ million investment in phases I\&II during 2009-2014, over 1 million samples from 150,000 patients were collected \& stored separately and the minimal data sets were uploaded regularly to the virtual central repository. BBCR now supports a variety type of research projects.

Statement of the Problem: The decentralized centers varying in capacity of storage places and capability of quality management, biospecimen hardly shared due to undefined sharing policy, and loss of employees, etc., will directly affect the sustainable development of the BBCR.

Proposed Solution: Therefore, BMSTC launched the phase III project of BBCR in May 2019, mainly aiming to realize the sustainable development and sharing of important clinical biospecimen from major diseases in Beijing through an independent third-party service platform, the Beijing Biobank of Life Science Park (BoLSP), which is located in ZhongGuanCun Life Science Park. BoLSP contains both a 1700 sq meter vapor phase liquid nitrogen storage facility and a 1700 sq meter experiment center. Thirteen BBCR member centers are invited to share their biospecimens with the central biobank BoLSP, along with clinical data, contributing to a large clinically annotated biospecimen repository network.

In the phase III project, the proposed solution includes proposals for establishment of biospecimen sharing mechanisms for utilization of resources, quality management system covering evidence-based standard operating procedures knowledge base to build uniform technical standards, information management system to integrate biobanking process with cost and quality control, and sustainable operation mode of the thirdparty platform to ensure a demand-driven repository to qualified customers.

Conclusion: The phase III BBCR will promote collaboration between BoLSP and regional member biobanks to form a large proactive inventory biorepository in North China with improved turnover and sustainability.

\section{PB-12 “Network Distributed Biobank": An Effective Approach To High-Quality Clinical Biobanking}

H. Yu ${ }^{1}$, B. Zhang ${ }^{1}$, B. Qin ${ }^{1}$, R. Zheng ${ }^{1}$, D. Cai ${ }^{1}$,

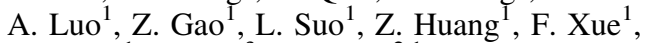
M. Zhou ${ }^{1}$, Z. Zhu ${ }^{3}$, J. Huang ${ }^{2,1}$

${ }^{1}$ School of Basic Medical Science Central South University, Changsha, China, ${ }^{2}$ School of Life Sciences, Central South University, Changsha, China, ${ }^{3}$ Sino-European Biological Resource Share \& Development, LLC, Changsha, China

There are abundant clinical resources in China. A survey of more than 10,000 clinical researchers from large comprehensive hospitals nationwide showed that almost every clinical department has its own biobank. More than $90 \%$ of studies of the biospecimens are focused on major clinical diseases. Many of these express a commitment to share in order to facilitate research efforts. However, how to develop an approach to facilitate the use of data and biospecimens efficiently is very important for us.

In the face of above problems, we created a "Network Distributed Biobank." The basic unit can be a nitrogen tank or a refrigerator, which is connected through the network platform. We assist clinical researchers build standardized sample frontend acquisition modules in the operating room, pathology department and laboratory department. The entire sample procedure management system is controlled by standards and standard operating procedures which are embedded in the platform system. The satellite biobank and central biobank, which we have already built, can support the operation of distributed units. At the same time, we cooperate with clinical researchers to input the deidentified clinical data onto the platform. Under uniform standards, biospecimens can be effectively shared and more quickly and effectively used.

Compared with a traditional biobank, the "Network Distributed Biobank" is a form of biobanking that is becoming more prominent. This approach helps clinical researchers obtain highquality biospecimens at lower cost and higher efficiency, and we believe it will become more widely implemented in the future.

\section{PB-13 McCain Genito-Urinary BioBank (MGB) at the University Health Network, Toronto, Ontario}

\section{S. Paul, H. Wagner, M. Kenk, N. Fleshner}

Surgical Oncology, University Health Network, Toronto, Ontario, Canada

Background: The McCain Genito-Urinary BioBank (MGB) was founded in 2008 as GU BioBank at University Health Network, a large tertiary health centre in Toronto, Ontario affiliated with the University of Toronto. The goal of MGB is to support biomarker discovery by collecting samples and metadata longitudinally across disease states and treatment lines.

Method: MGB research staff have mapped relevant clinical states for urologic cancer cohorts and controls and established a systematic and clinically-integrated approach to biospecimen procurement. Samples are collected at key steps, including 
diagnosis, disease recurrence, and at changes in treatments. This permits the collection of high-quality specimens and comprehensive standardized clinical data in a longitudinal fashion.

Liquid biospecimens (blood and urine) are collected at predetermined time points, catalogued in the specimen database, processed, bar-coded, and stored in liquid nitrogen vapor phase freezers for long-term banking. The specimen inventory and clinical database within the urology division are federated with information from the institutional electronic patient record and used for specimen selection and determination of clinical outcomes for studies approved by the institutional research ethics board.

Result: Since 2008, over 500,000 biospecimens have been collected, processed, and stored at MGB from 16,000 participants consented at five clinical sites in Toronto. We have banked over 145,000 serial collections of plasma and serum, close to 110,000 urine specimens, and over 30,000 buffy coat specimens. Specimens have been utilized in more than 50 projects, including proteomic, genomic, and epidemiologic studies performed through regional, national, and international collaborations. Research conducted generally focused on development and optimization of novel biomarkers, with the aim of improving diagnosis and personalizing treatment for genitourinary diseases, as well as facilitating the development of novel treatments.

Conclusion: Availability of high-quality biospecimens and associated clinical data translates to high-quality research outcomes, which in turn aid biomarker discoveries in oncology research. Ongoing refinements of cost-recovery models, development of research collaborations at national and international levels, and upgrading of informatics systems are critical for sustaining the growth of MGB and enhancing utilization of stored samples.

\section{PB-14 Clinical Data and Biobank Resource of the Beijing Friendship Hospital, Capital Medical University}

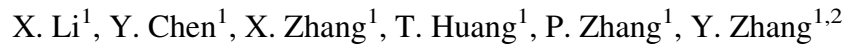

${ }^{1}$ Beijing Friendship Hospital, Capital Medical University, Beijing, China, ${ }^{2}$ Research Center, Beijing Clinical Medicine Institute, Beijing, China

Background: Clinical Data and Biobank Resource of the Beijing Friendship Hospital, Capital Medical University, was established in 2014. It is a hospital-based platform biobank, serving all the scientists in our hospital. It supports the construction of all disciplines in the hospital with the characteristics of digestive diseases. The preserved sample types and diseases are rich and diverse. It has become an important part of clinical research, basic scientific research, precision medicine, and translational medicine research in the hospital.

Methods: Our biobank covers an area of more than $500 \mathrm{~m} 2$ and has a storage capacity of one million. We have a team of seven (six full-time and one part-time) employees. At present, all of them have obtained the pre-job training certificate for biobanking. Our biobank has accumulated some experience in platform-based biobank management, information construction, standardization, and training and processing. Not only do we offer basic services such as sample collection, processing, storage, and disposal, but we also provide project consulting, integration of legacy samples, and several education projects as well.

Results: 2014-2016 was the construction and growth period of our biobank. 2017-2019 was the establishment and operation period of our quality management system. After 2020, our main focus will be on quality and sample sharing. We got ISO 9001:2015 certification in 2016 and have been operating under this system up to now. As of September 2019, a total of 91 disease sub-biobanks were built. Over 30 clinical departments have been served, more than 50 scientific research projects have been supported, and the total number of biological samples reached 350,000. We have also undertaken graduate teaching and continuing education training programs. We developed our own utility system in 2017 and upgraded according to ISO 20387 and the Regulations on the Management of Human Genetic Resources in China was issued.

Conclusions: As a hospital-based platform biobank of a general hospital, our biobank has the characteristics of rich disease types, diverse sample types, outstanding key disciplines, and standardized management. Being conscious of the key characteristics and service portfolio are critical requirements for the sustainability of a biobank.

\section{PB-15 Establishing a Biobank: The Challenges in a Resource Limited Settings}

P. Tanwar

\section{Laboratory Oncology Unit, Dr Bra-irch, All India Institute} of Medical Sciences, New Delhi, India

Statement of the Problem: The accelerated dynamics of targeted drug therapy research and personalized medicine has given a chain reaction start to a holistic concept called biobanking. The concept existed earlier and has been well established within developed nations; however, in low-and middle-income countries (LMICs), it is still a challenge. The importance of establishing a biobank is overwhelming for both researchers and administrators to realize. This will provide us the vital human tissue which is required for all translational research with the objective of achieving greater dimensions for diagnosis, treatment, and prevention. This will also help with molecular classification of the disease based on targeted therapy.

Proposed Solution: The concept of biobanking is at an infantile stage in LMICs countries. As we all are living in the era of a cancer epidemic, we have to establish and maintain a tissue repository which will sustain future drug and diagnostic biomarker research. This will also help us to carry out large drug trials and validation of diagnostic assays in a limited time period with the same efficiency. The role of the pathologist cannot be undermined in such a setting, where there are many important "issues of tissue" involved. The challenges, which pathologists and lab sciences workers face in a initiating and establishing a biobank at an optimally functional mode, are enormous right from administrative to scientific levels. There is always an impending need of persistent team effort, from the very initial stage of infrastructural blueprints to the ultimate stage of planning research trials. The maintenance of quality assured properly annotated samples along with their clinical data is very critical. The outcome of all ancillary experimental modalities including molecular/drug-related/genetic studies will depend on the viability of stored tissue. The high amount of funds required to start such a facility is also one of the ratelimiting constraints, especially in LMICs, which needs to be overcome. Being in under/limited-resourced settings, we have even more obligations to strictly comply with ideal practice to prevent the loss of revenue utilized to establish such a facility

Conclusion: Biobanking is not just a mere storage of tissue but a conceptual science, which clinicians, researchers, and administrators need to realize and would be the team effort which would help us execute this research science avenue. 
PB-16 Biobank Mississippi - A New Biobank Enriched For African Americans

J. E. Olson ${ }^{1}$, M. A. Hathcock ${ }^{1}$, G. D. Marshall ${ }^{2}$, J. R. Cerhan ${ }^{1}$, M. M. Resende ${ }^{3}$, S. J. Bielinski ${ }^{1}$, M. Cicek ${ }^{4}$, R. L. Summers ${ }^{2}$, G. J. Mahajan ${ }^{2}$

${ }^{1}$ Health Sciences Research, Mayo Clinic, Rochester, Minnesota, United States, ${ }^{2}$ University of Mississippi Medical Center, Jackson, Mississippi, United States, ${ }^{3}$ Center for Individualized Medicine, Mayo Clinic, Jacksonville, Florida, United States,

${ }^{4}$ Laboratory Medicine and Pathology, Mayo Clinic, Rochester, Minnesota, United States

Objectives: To report on development of a new biobank called "Biobank Mississippi" after three years of enrollment at the University of Mississippi Medical Center (UMMC).

Methods: Biobank Mississippi is a partnership between UMMC and the Mayo Clinic Center for Individualized Medicine Biorepository Program to encourage collaborations across institutions and to create a new diverse population of 10,000 persons for biomedical research. Potential participants are invited using marketing materials such as posters and brochures in clinic areas, screen-savers, and ads on the UMMC employee intranet. Recruitment sites include the UMMC Pavilion Clinic (Adult Outpatient); Rapid Care Clinic; Medical Mall in Jackson, MS; and locations in Lexington and Grenada, MS. Potential participants are current or former UMMC patients aged 18 years and older, able to provide written informed consent, and U.S. residents. Collected blood samples are processed into DNA, serum, and plasma. Patient surveys collect information on general health, personal and family health history, health behaviors, demographics, and education. Data are also available from electronic health records.

Results: After 2.5 years of recruitment, over 4,000 subjects have consented to the project and provided samples and data. The collection is targeted for completion by the end of 2021. Of those with completed enrollment, $76 \%$ self-reported being African American, 20\% reported being white, and $60 \%$ are female. The mean age is 44 years of age (standard deviation $=14$ ). Self-reported educational status included $15 \%$ without a high school (HS) education, $34 \%$ with HS only, $32 \%$ with some post-HS education, and $17 \%$ had $4+$ years of post-HS education. Most commonly selfreported conditions include hypertension (32\%), depression (25\%), anxiety (23\%), osteoarthritis (23\%), and gastroesophageal reflux (18\%). Human immunodeficiency virus (HIV) was reported by $14 \%$. A personal history of cancer was relatively common, with $4 \%$ reporting a history of breast cancer, $3 \%$ having a history of lung cancer, and $2 \%$ reporting a history of colorectal cancer.

Conclusions: Biobank Mississippi is a newly-established valuable resource for biomedical research projects within UMMC and Mayo Clinic as well as outside these systems. The access to this large well-annotated high quality samples may lead to innovative and personalized approaches that will contribute to advances in the precision medicine field.

\section{Biodiversity/Environmental/Animal Repositories}

\section{PC-01 NASA Institutional Scientific Collection (ISC) and Biospecimen Sharing Program at Ames Research Center}

R. T. Scott, A. J. French, A. E. Wood, D. K. Lopez, M. E. Del Alto, H. J. Stewart

Life Sciences Data Archive, NASA Ames Research Center, Moffett Field, California, United States
Space-flown non-human biospecimens are available for research. NASA's biospecimen collection represents a unique and limited resource of unique spaceflight payload and ground control research subjects. These specimens are harvested according to -established standard operating procedures that maintain their quality and integrity. Once the primary experiment objectives have been met, the remaining specimens are stored and made available to provide secondary opportunities for complementary studies or new investigations to broaden research without large expenditures of time or resources.

The NASA ISC at Ames currently stores flight and ground specimens from COSMOS, shuttle, and International Space Station (ISS) investigations. These specimens include bone, muscle, and organ tissues, as well as extracted DNA and RNA from mice, rats, bacteria, and quail. The samples are stored at $-80^{\circ} \mathrm{C}$ or $+4^{\circ} \mathrm{C}$, depending on fixative, for ideal preservation. Detailed metadata are available for all samples. Historically, these tissues have been used for a wide range of analyses, including histology, genomics, and transcriptomics.

NASA's current human space flight research is directed toward enabling human space exploration beyond low-Earth orbit. To that end, NASA Space Flight Payload Projects, Rodent Research, Cell Science, and Microbial Labs, flown on the ISS, benefit the global life sciences and commercial space communities. Verified data sets, science results, peer-reviewed publications, and returned biospecimens, collected and analyzed for flight and ground investigations, are all part of the knowledge base collected by NASA's Human Exploration and Operations Mission Directorate's Space Life and Physical Sciences Research and Applications Division, specifically the Human Research and Space Biology Programs. These data and biospecimens are made available through the public Life Sciences Data Archive (LSDA) website to promote basic discovery and pre-clinical and clinical science.

This poster will provide more information about available specimens and the LSDA biospecimen-sharing program. Website: https://lsda.jsc.nasa.gov/; https://www.nasa.gov/ames/ research/space-biosciences/isc-bsp

\section{PC-02 Effect of Quercetin on Lipid Peroxidation, Total Antioxidant Potential, Mitochondrial Activity, and Quality of Frozen-thawed Indian Red Jungle Fowl (Gallus gallus murghi) Sperm}

\author{
B. A. Rakha ${ }^{1}$, Q. U. Ain ${ }^{2}$, M. S. Ansari ${ }^{3}$, S. Akhter ${ }^{2}$
}

${ }^{1}$ Department of Wildlife Management, Pir Mehr Ali Shah Arid Agriculture University Rawalpindi, Rawalpindi, Punjab, Pakistan, ${ }^{2}$ Department of Zoology, Pir Mehr Ali Shah Arid Agriculture University Rawalpindi, Rawalpindi, Punjab, Pakistan, ${ }^{3}$ Department of Zoology, Division of Science and Technology, University of Education, Lahore, Punjab, Pakistan

The study was designed to elucidate the effects of quercetin on lipid peroxidation, total antioxidant potential, mitochondrial activity, and quality of frozen-thawed Indian red jungle fowl (Gallus gallus murghi) sperm. For this, semen was collected from seven adult Indian red jungle fowl through abdominal massage, and evaluated for volume, concentration, and motility. The qualifying semen ejaculates having $>80 \%$ motility were diluted in red fowl extender having quercetin $0 \mathrm{mM}$ (control), $5 \mathrm{mM}, 10 \mathrm{mM}, 15 \mathrm{mM}$, and $20 \mathrm{mM}$. Diluted semen was frozen following glycerol-based protocol and semen microscopic (motility, plasma membrane integrity, viability, acrosome, and 
chromatin damage) biochemical (mitochondrial activity and ferric reducing antioxidant power) and oxidative parameters (malondialdehyde) were determined after thawing at $37^{\circ} \mathrm{C}$. Motility and acrosome and chromatin integrity were recorded highest with $15-\mathrm{mM}$ and $20-\mathrm{mM}$ quercetin compared to quercetin $5 \mathrm{mM}, 10 \mathrm{mM}$, and control. Nevertheless, plasma membrane integrity, viability, mitochondrial activity, and antioxidant potential were recorded highest with $15-\mathrm{mM}$ quercetin compared to all experimental extenders. Malondialdehyde concentration in sperm and seminal plasma were recorded lowest $(\mathrm{P}<0.05)$ in extender having $15-\mathrm{mM}$ quercetin. It is concluded that quercetin supplementation in red fowl extender improve motility, mitochondrial activity, plasma membrane, and acrosme and chromatin integrity by elevating the total antioxidant potential and ameliorating the lipid peroxidation in frozen-thawed semen.

\section{PC-03 Use of the Livestock Biobanks for Food and Economic Security and Indigenous Species Conservation in Uganda}

\section{H. Ndagire}

National Animal Genetic Resources Centre \& Data Bank, Kampala, Uganda

Uganda is endowed with biodiversity owing to its yearround suitable tropical climate. It is also privileged with fertile soils and fresh water among diverse areas in the country. Uganda has several indigenous animal breeds among the different species of livestock kept. The indigenous animal breeds are characterized by lower production and productivity, calling for a need to perform cross breeding with the exotic livestock breeds. Cross breeding resulted in offspring with increased production and productivity, and thus production of more food and excess for sale, bringing enhanced food and economic security. The exotic breeds have exhibited limited adaptation to the heat, diseases, and their vectors. Indigenous animal species have several traits such as disease resistance, heat tolerance, ability to walk long distances, and ability to feed on wild pastures without supplementation, which brings about calls for conservation of their genetics.

Biobanks were formed for purposes of cross breeding and also conservation of indigenous animal breeds. Assisted reproductive technologies such as artificial insemination, multiple ovulation and embryo transfer, and non-pregnancy diagnosis are being employed at the National Animal Genetics Resources Centre and Data Bank (NAGRC\&DB) in order to facilitate use of samples from the Biobank at NAGRC\&DB. Samples such as semen and embryos are collected, processed, and then preserved in the biobank under liquid nitrogen; they are thereafter removed for use on the national herds. Tissue is also collected from the different livestock species with the objective of extracting DNA. DNA is also collected from the samples for molecular characterization of the animals. DNA is also stored in cryobanks for current and future use.

There is need to integrate data between the phenotypic and molecular characterization of the animals from the different breeds, thus enabling meaningful analysis from the bio repositories. This will facilitate meaningful utilization of samples in the biobanks. A need to adopt software for handling the massive data collected alongside the samples is highly sought.

Cross breeding between indigenous and exotic livestock breeds has enhanced livelihoods among several families in Uganda; however, the need to conserve the adapted indigenous breeds variety cannot be under estimated. Creation of functional cryobanks can strike the balance between cross breeding and conservation since all the genetics can be kept appropriately in the cryobanks.

\section{PC-04 Assessment of Arthropod Vector Usage from NIAID's BEI Resources Biorepository - Supporting Vector Control, Development, Epidemiology, and Pathogen Transmission Research}

\section{R. Bradford, T. Stedman}

\section{Federal Solutions, ATCC, Manassas, Virginia, United States}

Statement of the Problem: Infectious diseases spread by arthropod vectors represent a major cause of morbidity and mortality across the world. In spite of years of effort, effective vaccines have not been developed for routine immunization against many vector-borne pathogens. In addition, the lack of effective methods to control the transmission of these pathogens contributes to the burden on public health.

Proposed Solution: The Biological and Emerging Infections Resources Program (BEI Resources) was developed by the National Institute of Allergy and Infectious Diseases (NIAID) as a centralized bioresource to the global public health scientific community (www.beiresources.org). The Vector Resources collection currently provides live arthropod vectors from over 60 colonies of mosquitoes as well as Ixodid and soft ticks, sand flies, and triatomines. These vectors are competent hosts for transmission of blood-borne arboviruses, and parasitic and rickettsial diseases including dengue, Zika, Rocky Mountain spotted fever, Lyme borreliosis, Chagas, babesiosis, and malaria. We assessed 524 publications over the period 2011-2018 from investigators utilizing arthropod vectors and associated reagents from BEI Resources to determine scope of use. The fields of study were assigned to broad categories, consolidating subject indices and keywords to emphasize end use.

Conclusion: Based on context, the highest-ranking scope of use for BEI Resources vector materials included Vector Control topics (e.g., insecticide resistance, prevention, drug effects, and bioassays), and comprised nearly half of associated publications. Collection usage field of study was followed in descending order by Growth and Development; Genetics and Transcriptomics; Pathogen Transmission; and Immunity and Epidemiology. BEI Resources hosts freely-downloadable insectary protocol manuals for multiple arthropod vectors, providing a rich array of insectary management, safety, and experimental approaches for Vector Biology. These resources are available free of charge to registered users in domestic and foreign institutions in order to further support vector-borne disease research.

\section{PC-05 Center for Fungal Genetic Resources (CFGR): Housing Plant Pathogenic Fungi and Their Genomes}

Y. Yoon ${ }^{1}$, Y. Lee Le $^{2,1}$

${ }^{1}$ Center for Fungal Genetic Resources, Seoul National University, Seoul, Gwanak-ro Gwanak-go, Korea, ${ }^{2}$ Department of Agricultural Biotechnology, Seoul National University, Seoul, Korea

Fungi are eukaryotic organisms of ecological and industrial significance. Ecologically, they colonize a wide range of habitats and play essential roles in the ecosystem, particularly in the 
decomposition of organic matters. They have been used as food sources and agents of fermentation and for the production of various antibiotics and enzymes that are used in research, industry, and medicine. In contrary, the impact of many pathogenic fungi on animals and plants is economically and socially detrimental. For example, Magnaporthe oryzae causes one of the most destructive crop diseases, the rice blast. Annual yield loss by rice blast is equivalent to the amount that could feed about 60 million people. The Center for Fungal Genetic Resources (CFGR) was established to collect, maintain, and distribute genetic resources mainly from plant pathogenic fungi. These efforts are important for both educational and research purposes. Primarily, CFGR is a valuable resource center to assist the development of new strategies for management of crop diseases and other research in applied sciences. CFGR harbors a large collection of important fungal species: a total of 42,000 isolates from 59 species of fungi including 20,902 T-DNA transformants of the rice blast and anthracnose fungi. Endophytic mycobiome strains are also being collected and their potential as secondary metabolite produces will be measured. In addition to the biological materials, CFGR has developed user-friendly databases to maintain genetic information of fungal stocks and help to solve questions on fungal pathogenicity, population genetics, development, and evolution. Also, CFGR seeks strategies for sustainable and scientific plant quarantine to better protect our ecosystem from invasive microorganisms.

\section{PC-06 Biobanking in Livestock: Integrating DNA Technology for Small-holder Farmers}

\section{P. O. Soma, B. B. Kooverjee, G. Hutang, H. Swart}

Animal Breeding and Genetics, Agricultural Research Council, Pretoria, Gauteng, South Africa

Stock theft remains a major challenge in South Africa (SA), affecting all sectors of the farming community. Gauteng, the smallest province in SA, has shown a trend in the number of stock theft cases increasing over the past five years. The largest increase of $20.7 \%$ stock theft cases was reported for the 2016/ 2017 period. The impact of stock theft on resource-poor and small-scale farmers is often an order of magnitude more severe than for the commercial farmer due to the fact they often own small numbers of animals. These owners also in many cases do not have the financial means to make a rapid recovery following theft and subsequently the sustainability of their farming practices are threatened. Another cause of concern is that stolen animals or their products may also eventually enter the official commercial trading arena. Despite laws regulating the branding of animals, a number of livestock remain unidentified. However, the Livestock Identification Catalogue (LidCat ${ }^{\mathrm{TM}}$ ) can serve as a deterrent to stock theft in targeted areas and thus alleviate or reduce the financial losses incurred by resourcestrained farmers. The LidCat ${ }^{\mathrm{TM}}$ system was initially developed for the South African Police Services, Stock Theft Unit, as a repository for hair samples. It is aimed at reliable and affordable storage of hair samples of individual animals for future use. The system is based on collecting a hair sample from an animal, allocating a unique barcode and reference number to it (similar to an ID number for humans), and storing the sample under controlled conditions in the laboratory (biobanking). The approach is a powerful and accurate means of positive identification available and acts as an independent control for authenticating conventional or even newer technologies aimed at identification. It can be applied to establish whether the original form of identification has been tampered with or changed in any way during the period between first and secondary sampling. DNA-based methods have the potential to provide the necessary evidence to establish individual identification, ownership, and parentage verification. DNA technology is being used as an important forensic tool to resolve stock theft in the country and is increasingly becoming vital in ensuring accuracy and fairness in the criminal justice system. The integration of DNA technology in the farming systems in Gauteng has potential to decrease the incidence of stock theft in this province.

\section{Biospecimen Research and Science}

\section{PD-01 Archival Sample Submission for Clinical Trial Enrollment: One Biorepository's Experience}

\section{K. E. Soafer, E. Knutson, A. L. Coulas, S. J. McCall \\ Department of Pathology, Duke University, Durham, North Carolina, United States}

Background: Duke University Health Systems (DUHS) supports multiple interventional patient-based clinical trial requests. The BioRepository and Precision Pathology Center (BRPC) facilitates over 80 of these protocol-specific trial-based treatments. Since institutional policies prohibit the depletion of limited archived tissue samples per federal law, DUHS BRPC has offered an alternative to whole block submission. In order to minimize the prospect of depletion regarding archived samples, BRPC has offered unstained slides, punches, and re-embedded cores.

Methods: The DUHS BRPC facilitated clinical trial requests by creating and distributing FFPE blocks, cores, scrolls, and/or slides to investigators. The BRPC retrospectively reviewed 34 months of researcher requests for these retrospective samples. Data were retrospectively analyzed in 12-month increments to evaluate the trends in how researchers requested tissue (slides, scrolls, FFPE blocks, or cores), if the request drove patient trialrelated treatment or not, and if the researcher originally requested an FFPE block but accepted slides or cores instead.

Results: From 2017 to October 2019, 814 clinical trial requests were fulfilled by BRPC with 593 cases driving patient trial-related treatment and 221 not. The breakdown of the requested retrospective samples per year: 2019: 28 cores, 0 FFPE blocks, 133 slides, and one scroll; 2018: 58 cores, 16 FFPE blocks, 238 slides, and six scrolls; 2017: 18 cores, 12 FFPE blocks, 299 slides, and five scrolls. Because of a policy prohibiting release of entire archival blocks, 24 cases originally requested an FFPE block but a core was accepted instead. Similarly, 30 cases originally requested an FFPE block but slides were accepted instead.

Conclusion: The BRPC sees twice as many requests where patient trial-related treatment is at stake than where it is not. Per institutional policy, limited archival samples being requested to drive trial-related treatment can be exhausted, unlike limited archival samples which are being requested by the sponsor for "correlative science" rather than treatment decisions. Institutional policy prohibits release of original paraffin blocks in compliance with federal law. However, BRPC offers unstained slides or punched and re-embedded tissue cores as alternatives. Over the 34 months, a total of $7 \%$ (55/814) of requests asked for the original block and BRPC offered another solution via cores or slides. 
PD-02 Effect of Cold Ischemia on RNA Integrity of Tumor Tissues: A Meta-analysis

H. Li, N. He, Y. Guo

Cancer Biobank, Tianjin Medical University Cancer Institute and Hospital, Tianjin, China

Cold ischemia time appears to be a risk factor for RNA quality which affects downstream analyses. However, the association between cold ischemia time and RNA quality on tumor tissues has been inconsistent between studies. To clarify the association of cold ischemia time and RNA quality which may help to inform decision making in the evidence-based standards, we performed a meta-analysis of clinical studies comparing RIN values in cancer patient tissues at different cold ischemia time.

Data Sources: PubMed and EMBASE electronic databases were searched between 2007 and January 2019, including references of qualifying articles.

Study Selection: We included English-language, original investigations in human cancer with RIN values. Titles, abstracts, and articles were reviewed by at least two independent readers. Eight of 234 articles that had 990 cancer tissues met the inclusion criteria, assessed RIN values at different cold ischemia time.

Data Extraction: One reviewer performed a full abstraction and another reviewer verified accuracy. We contacted authors and obtained additional information for three articles with insufficient reported data. Using the primary data from each of the studies, we calculated study-specific relative risk (RR) that were combined using a random-effects model. Heterogeneity was assessed using the Q statistic and I2. Subgroup analyses were performed for the cancer type, the organ type, and the cancer number.

Results: We identified eight studies investigating RNA quality after different cold ischemia time at room temperature. Eight studies reported cold ischemia time ( $\leq 30 \mathrm{~min}$ and $>30 \mathrm{~min}$ ) and the corresponding RIN values $(\geq 5$ and $<5)$; a meta-analysis demonstrated that cold ischemia time $>30 \mathrm{~min}$ had no significant impact on RIN values (RR, 1.059; 95\% confidence interval, $0.908,1.234$ ) of tumor tissues. Results were consistent in all subgroups.

Conclusion: The result indicates that cold ischemia time $>30 \mathrm{~min}$ did not increase the risk of cancer tissue degradation. Further research should be further investigated about whether this association is causal and establish a precision standard for times of cold ischemia time.

\section{PD-03 RNA Integrity and Histological Assessment of Frozen Hepatocellular Carcinoma Tissue Samples}

X. Zhang, A. Lin

Taizhou Hospital of Zhejiang Province, Linhai, China

Background: Hepatocellular carcinoma (HCC) is the fifth most common cancer worldwide, and the third most common cause of cancer mortality. To make materials available for the basic and translational research for HCC, some liver cancer biobanks have been constructed. In order to collect high-quality biospecimens, standard operating procedures on how to collect, operate, store, transport, and use must be set up. The histological and molecular quality of the collected biospecimens should be assessed in a routine way.
Methods: Hepatocellular cancer tissues were collected by standardized mirror image method at the Taizhou human tissue bank in China. For quality evaluation, fresh-frozen HCC tissues from 79 patients were randomly selected for histopathology examination and the tumor cell content was determined. Among these samples, 52 liver cancer tissues with the tumor cell content higher than $70 \%$ were used for RNA integrity detection. The effect of storage periods on RNA integrity and the association of RNA integrity number (RIN) values with clinicopathological parameters were analyzed.

Results: Eighty-one percent of liver cancer tissue specimens were observed over $70 \%$ tumor cell content in histological assessment; $92.3 \%(n=48 / 52)$ of banked liver cancer tissues yielded high-quality RNA with RIN $\geq 7$ and a mean of $8.15 \pm 0.60$. Long-term storage periods of banked HCC tissues did not affect RNA integrity negatively $(\mathrm{P}=0.226)$. Moreover, a significant association was observed between RIN values and tumor node metastasis stage of HCC patients $(\mathrm{P}=0.025)$.

Conclusions: High-quality liver tissue specimens were collected at the Taizhou human tissue bank. RNA in frozen liver tissue specimens is stable. These results highlighted that the banked liver tissue samples procured by the guidance of standard operating procedures can provide a valuable resource for liver cancer research.

\section{PD-04 High-Performance Liquid Chromatography (HPLC) Analysis and Antioxidants Activities of Extract of Azadirachta indica (Neem) Leaves}

W. K. Darkwah, E. Biney, J. Puplampu

University of Cape Coast, Cape Coast, Ghana

Background: Extracts from Azadirachta indica tend to provide numerous health benefits including antioxidant activity. The main objectives for this research was to use standard procedures to determine total phenols, total alkaloids, total flavonoid, DPPH - scavenging activity, ferric reducing power, total antioxidant capacity, and analyze active components of the extracts using high-performance liquid chromatography (HPLC).

Method: In vitro antioxidant potential of the Azadirachta indica extract was evaluated using DPPH and total antioxidant ability assays. Ferric reducing power ability of the extract was also examined using tannic acid and ascorbic acid as standard. Concentrations of plant extracts ranging from 0.02 to $0.10 \mathrm{mg} / \mathrm{mL}$ were prepared and mixed with appropriate volumes of reagents.

Results: Methanol extract of Azadirachta indica exhibited higher content of phytochemical compounds (alkaloid $=1.9 \mathrm{mg}$ $\mathrm{QE} / \mathrm{g}$; flavonoids $=3.5 \mathrm{mg} \mathrm{QE} / \mathrm{g}$; and phenols $=4.9 \mathrm{mg} \mathrm{QE} / \mathrm{g}$ ) at concentration $0.1 \mathrm{mg} / \mathrm{mL}$ compared to the acetone/water extract (alkaloid $=1.7 \mathrm{mg} \mathrm{QE} / \mathrm{g}$; flavonoids $=1.4 \mathrm{mg} \mathrm{QE} / \mathrm{g}$; and phenols $=2.6 \mathrm{mg} \mathrm{QE} / \mathrm{g}$ ). An over-all trend found in the present study highlights the fact that the methanol extracts have better antioxidant capacities (DPPH, total antioxidant ability and FRAP) than the acetone/water extract. HPLC analysis conducted also reveals seven peaks for methanol extract and six for acetone-water extract with different heights.

Conclusion: HPLC analysis of Azadirachta indica extracts exhibited the presence of azadirachtin compound. The study showed that the extracts can competently protect the body against oxidative stress; therefore, they can be used as a source of potent natural antioxidant compounds. 
PD-05 Transcriptome Analysis of Common and Diverged Circulating MiRNAs between Arterial and Venous During Aging

H. Wang ${ }^{1}$, L. Xue ${ }^{1,2}$

${ }^{1}$ Medical Research Center, Peking University Third Hospital, Beijing, China, ${ }^{2}$ Biobank, Peking University Third Hospital, Beijing, China

Background: ncRNA has been one of the targets for further research in biomarkers hunting in many biobanks. Although peripheral venous blood was highly focused on as clinical biomarkers, arterial miRNAs exhibited slightly different expression profiles. We compared the miRNA profiles of venous- and arterial-derived plasma between young and aged male rats, in order to explore whether venous miRNAs can represent entire circulating vessels in the given conditions like aging.

Methods: The miRNA profiles were obtained by Illumina next-generation sequencing. Differentially expressed miRNAs were obtained by DEGseq tool and validated by quantitative real-time PCR. MSigDB Hallmark Gene Set reference and TAM 2.0 server were used to investigate the enriched functions and associated diseases. TransmiR v2.0 tool was used for enriched upstream transcription factors.

Results: We obtained the plasma miRNA profiles from abdominal aorta or abdominal main vein of young or aged rats (GSE138317). Two thirds of aging-related de-regulated miRNAs in vein showed no difference in artery. Even between artery and vein from the same individual, the miRNA expression profiles differed. We observed 54 and 42 arterial-versus-venous differential-expressed miRNAs in young and aged groups, respectively, exhibiting the different miRNA profiles from varied plasma origin under normal condition. These differences may be amplified to the same direction or switch to the opposite direction under various physiological or pathological conditions like aging. Only a few arterial-venous differential-expressed miRNAs shared between the young and aged groups. miR-450a/b and miR-223 showed the similar tendency, while five miRNAs, including miR136 and miR-503, displayed the opposite direction under the same scenario. We further analyzed upstream regulators which influence miRNAs level for plasma origin and found enriched transcription factors like NF- $\mathrm{BB}$ and SIRT1. These transcriptional factors could be organ-specific expression and/or regulated in physiological and aging states as parts of plausible causal factors.

Conclusion: This study indicated the influence of origin for plasma miRNAs upon certain a scenario and its potential regulatory rule, suggesting the potential application of circulating miRNAs for reflecting the systematic response of the body. Therefore, our data remind each biobanker that the origins have to be considered in the ncRNA research once sampling.

\section{PD-06 Systematic Analysis of Plasma Biomarkers for the Early Detection of Nasopharyngeal Carcinoma}

S. Vijaya Mohan ${ }^{1,2}$, G. Tan ${ }^{1}$, H. Alia Aina ${ }^{2}$, W. Kim ${ }^{2}$, H. Sathasivam ${ }^{2}$, A. Khoo ${ }^{1}$, L. Tan ${ }^{1}$

${ }^{1}$ Molecular Pathology Unit, Institute for Medical Research, Ministry of Health, Kuala Lumpur, Malaysia, ${ }^{2}$ BioBank Unit, Institute for Medical Research, Ministry of Health, Kuala Lumpur, Malaysia

Background: Nasopharyngeal carcinoma (NPC) is an epithelial cell malignancy that arises at the back of the nose at the base of the skull. The exact cause of the tumour is unknown, but it is highly associated with Epstein-Barr infection. The majority of countries with the highest NPC incidence and mortality rates are low-and-middle-income countries (LMICs). NPC is endemic in southern China and South East Asia, particularly Vietnam, Indonesia, and Malaysia. Malaysia is a multi-ethnic country with age-standardised rate of NPC ranging from less than 1 per 100,000 (Malaysian Indians) to more than 30 per 100,000 (Malaysian Chinese, Bidayuh, and Kadazan natives). Plasma biomarkers for early detection of NPC had been established from many southern Chinese cohort studies, but it is unclear if these biomarkers would be applicable to other populations. A cancer biospecimen bank (BioBank) was established at the Institute of Medical Research (IMR), Malaysia, which moderates collection of biospecimens. By utilising retrospectively collected blood samples, we have systematically analysed six established and four new plasma biomarkers that have the potential to screen local NPC for early diagnosis.

Methods: Sample donors were consented and recruited by the IMR BioBank. Blood samples were fractionated into plasma and stored according to standardised procedures. This study analyzed 232 NPC samples and 245 population and hospital controls. The biomarkers analysed were BamHI-W $76 \mathrm{bp}$, BamHI-W 121 bp, EBNA-1 99 bp, EA IgA, EA IgG, EBNA-1 IgA, VCA IgA, ebv-BART-miR-17, hsa-miR-29a, and hsa-miR-103.

Results: Despite NPC being detected at late stage, the repository acquired 30 stage I patients, which is higher than other current reported studies. We show that BamHI-W 76 bp levels are $97.4 \%$ sensitive and its specificity is $94.2 \%$ against population controls and $90.4 \%$ against hospital controls. Decision tree modelling shows combination of BamHI-W $76 \mathrm{bp}$ and VCA IgA or EA IgG may increase the specificity or sensitivity to detect NPC. EBNA1 99 bp could identify NPC patients with poor prognosis in early and advanced stage.

Conclusion: Our findings provided evidence for improvement in NPC screening strategies, combining biomarkers to increase sensitivity or specificity and testing biomarkers from single sampled specimen to avoid logistic problems of resampling. This will provide LMIC policymakers who have limited resources to plan more cost-effective screening and monitoring strategies.

\section{PD-07 Data-Driven Approach for Matching Samples with Downstream Applications}

\section{J. P. Wright}

Tissue Core, Moffitt Cancer Center, Tampa, Florida, United States

Statement of Problem: Recent advances in molecular technologies have allowed nucleic acid research to be performed using extractions from archived FFPE samples, thus significantly increasing the possible cohort numbers and the potential to identify clinically significant biomarkers. However, nucleic acid integrity criteria are specific to the selected downstream molecular application and may lead to a waste of resources when thresholds are not met. Our preliminary analysis of 2,303 RNA samples extracted from archived FFPE specimens indicates a correlation between nucleic acid integrity and time to analysis (FFPE block age) that differed based on tissue type. For example, RNA extracted from FFPE brain block yield better 260/230 values at eight and 14 years of age than lung and adrenal tissue type of same age and storage conditions. Additionally, RNA extracted from adrenal FFPE blocks had higher concentration then those extracted from brain, lung, or 
lymph node specimens. Finally, the data also suggested that adrenal samples yield higher ssRNA concentration then RNA extracted from brain, lung, and lymph node of the same block age. The relationship between FFPE tissue type, age of block, and requirements for certain assays needs to be examined on a greater scale.

Proposed Solution: We hypothesize that a data-focused approach, using collected data, can guide investigators' selection of research biospecimens by predicting compatibility of archived biospecimens for downstream molecular applications. One such explaination is using ischemia time, disease, and anatomic site to establish parameters based on the quality and quantity of nucleic acids. This could lead to the identification of specimens that might not meet the standard to preform downstream application and waste resources. To make the process more efficient, we propose selecting samples based on FFPE tissue type, age of block, and assay requirements. This will aid in matching samples to downstream applications, while still matching clinical outcomes.

Conclusion: Utilization and implementation of a database control metrics for sample extraction protocols may help identify outlying variables and provide a way to increase funding for biorepositories by giving customers the opportunity to compare samples against established baselines before moving to downstream applications. At the same time it can save the investigator time and money.

\section{PD-08 Single Lysis-salting Out Method of Genomic DNA Extraction for Dried Blood Spots}

K. Tumkur Puttasiddhappa ${ }^{1}$, M. Shaik ${ }^{2,1}$, M. Kamate ${ }^{3}$

${ }^{1}$ Department of Neurochemistry, National Institute of Mental Health and Neuro Sciences, Bengaluru, Karnataka, India, ${ }^{2}$ Department of Biotechnology and Microbiology, Karnatak University, Dharwad, Karnataka, India, ${ }^{3}$ Department of Pediatrics, Jawaharlal Nehru Medical College, KLE University, Belgaum, Karnataka, India

Background: Dried blood spots (DBS) are an important form of biopreservation and biobanking materials for storing blood samples for genetic studies. This has necessitated in developing an effective protocol to isolate genomic DNA (gDNA) from DBS samples. In this study, we have elucidated a dependable and non-hazardous "Single Lysis-Salting Out" (SLSO) protocol of gDNA extraction from DBS and compared against the available commercial kits.

Methods: For the purpose of this study, blood spots were collected on S\&S 903 filter cards from 10 healthy volunteers and 30 patients with glutaric aciduria type I (GA-I). The gDNA was extracted from these DBS samples by SLSO, QIAamp ${ }^{\circledR}$ gDNA Micro kit, and innuPREP forensic kit methods. The quantity and quality of gDNA obtained from these methods were determined by measuring the absorbance using a nanodrop spectrophotometer.

Results: The SLSO method showed four-fold and eight-fold increased yield of gDNA in healthy volunteers and patient samples, respectively, compared to commercial kits $(\mathrm{p}<0.0001)$. The protocol was also found to be cost efficient, reducing the per sample cost to almost half. The suitability of this method for genetic studies was confirmed by performing R402W genotyping by RFLP in GA-I patients. The genotyping results showed the presence of R402W mutation in $20 \%(6 / 30)$ of patients.

Conclusion: The SLSO method was found to be inexpensive, non-hazardous, and a suitable technique for isolating gDNA from DBS samples for genetic studies.

\section{PD-09 Pre-analytical Variables: Can They Influence the Success Rate of DNA Sequencing?}

\author{
L. Spary, F. Morgan, R. Clarkson, A. Parry-Jones \\ Wales Cancer Bank, Cardiff, S. Glamorgan, United Kingdom
}

Background: Histopathology of a patient's tumour is heavily reliant on the use of fixed tissue. Fixation methods vary between institutions, and although fixation ensures sample integrity, it also causes substantial chemical modifications to the DNA. During fixation, nucleotides are cross-linked with proteins and DNA is degraded into small fragments, all of which create issues in the isolation of DNA for genetic testing. In addition, the use of archived samples can also increase the complications. The Wales Cancer Bank (WCB), sent banked tissue samples for genetic testing and initial studies yielded high failure rates. In a bid to identify the causes, WCB undertook a project looking for correlations between sample preparation and qualities and the failure rates.

Methods: Formalin-fixed, paraffin-embedded (FFPE) samples were collected from more than 2,000 patients diagnosed with various solid cancers. For each sample the ischemic times and fixation process were recorded along with the operation date and date samples were sent for analysis. Sections were cut from each block and mounted onto glass slides before sending for analysis.

Results: Early results indicated that FFPE from certain tissue types are more difficult to sequence than others with prostate being the most problematic. The type of specimen, biopsy, or resection, did not influence the outcome of molecular analysis. As expected, higher concentrations of DNA were obtained from samples containing a higher percentage of tumour. Interestingly, low concentrations of DNA did not always result in failure but fewer failures were observed in samples with high percentages of tumour. Most importantly, the age of the sample and its length in storage had the biggest effect on the sequencing outcome. The effects of ischemic and fixation times on the sequencing outcome are currently being analysed.

Conclusions: Most studies comparing the molecular profile of tumours with outcome data use FFPE samples from patients with at least five years follow-up. Initial findings from this study indicate that the age of the sample is important and has a major influence on sequencing results. Correlations between the thickness of the tissue sections, the area of tissue, or the concentration of DNA $(n g / \mu l)$ with sequencing success rates are difficult to ascertain. With the increase in genetic testing for cancers, more work is needed to understand the preanalytical variables influencing successful sequencing of FFPE samples.

\section{PD-10 Differential Regulation of Immune-Related Genes between Right and Left Side Colorectal Cancer}

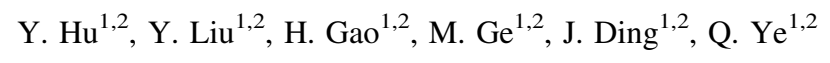

${ }^{1}$ Department of Pathology, Nanjing Drum Tower Hospital, The Affiliated Hospital of Nanjing University Medical School, Nanjing, Jiangsu, China, ${ }^{2}$ Nanjing Multi-center Biobank, Nanjing Drum Tower Hospital, The Affiliated Hospital of Nanjing University Medical School, Nanjing, Jiangsu, China

Background: The aim of this study was to define differences of immune-related genes in tumor microenvironment between right and left side colorectal cancer (CRC) and explore their therapeutic implications. Paraffin tissues and related information of CRC in biobank were mainly used. 
Methods: Gene expression profiling and clinical characteristics of patients with CRC were retrieved from TCGA. Nine genes were selected by gene differential expression analysis. To further validate the findings, we applied immunohistochemical (IHC) staining of a CRC tissue microarray. To reveal the prognostic value of differential genes on right-side colon cancer (RCC) and left-side colorectal cancer (LCC) patients, the survival analysis were performed. To study the function of the genes, GO and KEGG databases were used for analysis.

Results: We first analyzed the clinical and pathological information of 525 samples downloaded from the TCGA database that include 207 LCC and 318 RCC samples. We found that RCC and LCC were different in age, pathologic stage, mucus secretion, and microsatellite stability. In RCC, the proportion of patients over 70 years old was higher than that of LCC. N-0 cases were significantly more frequent than in LCC. The proportion of mucus secretion and MSI in RCC were higher. All the differences were statistically significant, $\mathrm{p}<0.01$. Gene differential expression analysis showed that the expression of seven genes in RCC was higher than that in LCC, which were CCR5, CD209, CD8A, HCK, HLA-DPB1, HLA-DQA1, HLADRA, respectively. Meanwhile, the expression of two genes in LCC was higher than that in LCC, which were IL-34 and PROCR. The genes were verified by IHC staining. Furthermore, survival analysis shows that patients with LCC have a better prognosis than RCC. Among all the differentially expressed genes, we found that HLA-DQA1 was highly expressed in RCC, and HLA-DQA1high patients had a better survival in RCC. IL-34 was highly expressed in LCC, and IL-34high patients had a better survival in LCC. Enrichment analysis of GO and KEGG indicated that these genes are mainly involved in immune response and $\mathrm{MHC}-\|$-mediated antigen presentation.

Conclusions: In this study, we comprehensively compared differences in the immune-related genes between right- and leftside CRC and explored the differences in gene expression and function and their impact on survival. Overall, we hope that our depiction of the differential genes of RCC and LCC could lay a foundation for further research.

\section{PD-11 Effect of Different Storage Conditions on Stability of Healthy Plasma-Derived miRNA}

R. labib $^{2,1}$, M. A. Elden ${ }^{2}$

${ }^{1}$ Laboratory of Cancer Biology and Genetics, NIH, Bethesda, Maryland, United States, ${ }^{2}$ Biorepository and Biospecimen Research, Children's Cancer Hospital Egypt 57357, Cairo, Egypt

Background: Plasma samples are usually stored in biobank repositories and are very appreciated by researchers due to the availability of different biomarkers as proteins, lipids, and micrRNA molecules. MicroRNAs are a class of small sequenced cellular RNAs consists of 21-23 nucleotides. MiRNAs have a vital role in gene expression as post-transcriptional regulators; as they are found in the bloodstream, miRNAs are intensively evaluated as promising blood-based biomarkers. This growing interest in developing assays for circulating miRNAs necessitates careful consideration of the effects of preanalytical and analytical parameters on the stability and quantification of circulating miRNAs.

Methods and Materials: In this study, we extracted micro RNA molecules by using MIRnase Kit (Qiagen) from the plasma of 11 healthy volunteers and stored it in different temperatures $\left(4^{\circ} \mathrm{C}, 20^{\circ} \mathrm{C}, 80^{\circ} \mathrm{C}\right.$, and liquid nitrogen) for different times ( 0,24 hours, 48 hours, and 3 months), then measured by nanoquant to determine the concentration of microrna from different storage conditions. To determine the expression level of mature miRNAs, we used quantitive real-time polymerase chain reaction (qPCR) using SYBER green (Qiagen) on an applied Biosystems 7500 real-time PCR machine (life technology for eight microrna [mir 146a, mir 451-5P, mir 122, mir 23a, mir 15b, mir 144-5P, mir 16-5P, mir 24]). All samples were analyzed in duplicate. Mean absolute CT values and standard deviations are displayed on the graphs. Statistics were performed using Student's t-test and $\mathrm{p}<0.01$ was considered significant.

Result: The expression of all eight microrna in the $-80^{\circ} \mathrm{C}$ stored plasma is significantly $(\mathrm{p}<0.01)$ higher than others that are stored in different temperature compared to fresh samples. microRNA concentration is also significantly higher in $-80^{\circ} \mathrm{C}$ stored plasma than other stored samples in liquid nitrogen, $-20^{\circ} \mathrm{C}, 4^{\circ} \mathrm{C}$, which has no significant value. To our surprise, the impact of short-term storage at $4^{\circ} \mathrm{C}$ on circulating miRNAs is much less than expected.

Conclusions: Data obtained in this study showed that circulating miRNA levels are unstable when plasma is stored at $4^{\circ} \mathrm{C},-20^{\circ} \mathrm{C}$, and samples should be kept at $-80^{\circ} \mathrm{C}$ and miRNAs are considered as biomarkers based on their stability under various storage conditions. This could be applied at the biobank repositories to optimize the storage conditions, and at the scientific advisory process of the researchers in their projects.

\section{PD-12 CircRNA Microarray Profiling Identifies a Novel Biomarker for Detection of Pediatric Acute Myeloid Leukemia}

X. Liu ${ }^{1,2}$, M. Cai ${ }^{2,3}$, H. Jiang ${ }^{2}$, L. Xu ${ }^{2}$

${ }^{1}$ Division of Birth Cohort Study, Guangzhou Women and Children's Medical Center, Guangzhou, China, ${ }^{2}$ Department of Hematology, Guangzhou Women and Children's Medical Center, Guangzhou, China, ${ }^{3}$ Institute of Pediatrics, Guangzhou Women and Children's Medical Center, Guangzhou, China

Background: Acute myeloid leukemia (AML) is a heterogeneous disease characterized by abnormal clonal hematopoietic progenitors. Despite trends in improved survival due to better supportive care, up to $40 \%$ of patients will have refractory disease, which has a poorly understood biology and carries a dismal prognosis. Circular RNAs (circRNAs), a large new class of endogenous noncoding RNAs that form covalently closed continuous loops without 5'caps or 3'tails, have recently been identified as potential functional modulators in cancer biology and are potential biomarkers and targets for cancer therapy. Until now, the expression patterns and roles of circRNAs in pediatric AML have remained largely unknown.

Methods: Profiles of circRNAs were analyzed using an Arraystar human circRNA microarray with seven bone marrow samples from patients with newly diagnosed AML and five from healthy donor as control. Then the expression of the top upregulated circRNA, circRNF220, was confirmed by quantitative reverse transcription PCR (qRT-PCR) in 118 primary AML patients. Short hairpin RNA mediated knockdown expression of circRNF220 were performed to prove the functional significance of circRNF220.

Results: CircRNA microarray analysis revealed that 506 circRNAs were differentially expressed in pediatric AML patients, with 382 circRNAs found to be upregulated and 124 to be downregulated. qRT-PCR showed that circRNF220 was signifcantly upregulated in pediatric AML, and $102(86.4 \%)$ AML patients displayed more than twofold upregulation in the circRNF220 expression level especially. Furthermore, the area under the ROC curve in distinguishing AML and normal ones 
was 0.915 , which yielded a $90 \%$ sensitivity, $100 \%$ specificity when using circRNF220 signal to diagnose AML independently. Short hairpin RNA mediated knockdown expression of circRNF220 combined with in vitro assays demonstrated that circRNF220 has pleiotropic effects, including promotion myeloid differentiation and inhibition of AML cell apoptosis. Using results of gene-expression microarray analysis, we found that expression levels of circRNF220 were inversely correlated with expression levels of predicted target genes encoding proteins involved in pathways of stem cell self-renewal and ubiquitination associated with protein degradation.

Conclusions: This study revealed a AML-derived circRNF220 played a vital role in myeloid leukemogenesis, and it could serve as a robust biomarker for detection of pediatric AML.

\section{PD-13 Addressing Unmet Needs in Alzheimer's Disease and Related Dementias Research Using a Multiple-Pronged Approach for Human Biospecimen Collection and Distribution}

M. VonDran, S. Shad, G. Kopen, T. J. Bell

NDRI, Philadelphia, Pennsylvania, United States

Background: As the most common cause of dementia, Alzheimer's disease (AD) is a devastating, progressive neurodegenerative disease that affects over 5.2 million Americans. As of yet, there are no disease-modifying treatment options available for $\mathrm{AD}$ patients. Human biospecimens are essential to advance our understanding of pathogenesis and treatment of $\mathrm{AD}$ and related dementias (AD/ADRD). Access to a diverse range of human biospecimens is essential to support the escalating nature of experimental techniques that $\mathrm{AD} / \mathrm{ADRD}$ investigators are pursuing. More comprehensive AD/ADRD human tissue resources are needed.

Methods: NDRI's nation-wide recovery network was used to screen and authorize AD/ADRD and normal control donors. Based upon specific, project-defined criteria, human biospecimens with relevant medical history were recovered and either shipped directly to approved investigators or to the AD/ADRD Human Biospecimen Resource (ADBR) biobank for storage and subsequent distribution to approved investigators upon request.

Results: Here we report the recovery results and structure of the ADBR. The ADBR is a new, NIH-funded resource that has the capabilities to provide project-specific and banked human biospecimens from two distinct AD/ADRD cohorts, living patients and post-mortem donors. Human brains and other human biospecimens were recovered from authorized donors according to investigator-specific or ADBR-specific recovery protocols. The breadth of biospecimen recoveries for ADBR was driven directly by an individual ADBR-approved investigator or highpriority needs that were identified by the ADBR advisory council, including enhancing donor diversity. To date, multiple donor-type and tissue-types have been screened and recovered to support $\mathrm{AD}$ research.

Conclusions: Analysis on human biospecimens provides the opportunity for translational studies on tissues and cells that are directly relevant to human pathophysiology of AD/ADRD. Here we report our efforts to address this unmet need. The advantages of the ADBR's combination approach are three-fold. 1) The post mortem donations permit the collection of a diverse pool of AD/ADRD donors and control (non-diseased) biospecimens; 2) the living patient collection enables acquisition of biospecimens that could capture unique time points, such as the patients' responses to given treatment protocols; and 3) standardization of donor eligibility and biospecimen collection methods will yield more accurate and reproducible data.

\section{PD-14 Precision Medicine in Childhood Cancer: From Tiny Biospecimen to Big Possibilities!}

T. Sontag ${ }^{1}$, S. Langlois ${ }^{1}$, A. Lefebvre ${ }^{1}$, S. Vairy ${ }_{5}^{2,3}$, C. Richer ${ }^{1}$, N. Piché ${ }^{4}$, D. Dal Soglio ${ }^{5}$, N. Patey ${ }^{5}$, B. Ellezam ${ }^{5}$, Y. Samson ${ }^{2,3}$, P. Teira ${ }^{2,3}$, I. Delorme ${ }^{6}$, B. Michon ${ }^{7}$, V. Larouche ${ }^{7}$, L. Naccache ${ }^{7}$, B. Desbiens ${ }^{7}$, D. Mitchell ${ }^{8}$, E. Anillo ${ }^{8}$, G. Cardinal ${ }^{9}$, T. Tran ${ }^{2,3}$, M. Marzouki ${ }^{2,3}$, D. Sinnett ${ }^{1,3}$

${ }^{1}$ Sainte-Justine UHC Research Centre, Montréal, Québec, Canada, ${ }^{2}$ Hematology-Oncology-Immunology Clinical Research Unit, Sainte-Justine UHC, Montréal, Québec, Canada, ${ }^{3}$ Department of Pediatrics, University of Montréal, Montréal, Québec, Canada, ${ }^{4}$ Surgery Department, SainteJustine UHC, Montréal, Québec, Canada, ${ }^{5}$ Pathology Department, Sainte-Justine UHC, Montréal, Québec, Canada, ${ }^{6}$ Hematology Unit, Sainte-Justine UHC, Montréal, Québec, Canada, ${ }^{7}$ Hematology-Oncology Clinical Research Unit, Québec UHC - Laval University, Québec City, Québec, Canada, ${ }^{8}$ Hematology-Oncology Clinical Research Unit, McGill UHC, Montréal, Québec, Canada, ${ }^{9}$ Research Ethics Committee, Sainte-Justine UHC, Montréal, Québec, Canada

Despite improvements in risk-stratified treatments, $\sim 20 \%$ of childhood cancer patients do not respond to current therapies and ultimately succumb to their disease. It remains the first cause of death by disease among children. However, no significant progress has been noted over the last decade, urging the need for new and more effective therapeutic alternatives. To address these issues, we have launched TRICEPS, a Childhood Cancer Precision Medicine program, which targets hard-to-treat pediatric cancers by offering in-depth multi-modal-omics investigation of tumour material to identify patient-specific alterations within a reasonable timeframe (eight weeks). One of the key components of TRICEPS was the availability of a biobank containing high-quality human biospecimens such as tumour material, blood, and saliva, along with well-annotated clinical data. Our biobank collected, preserved, and provided access in a transparent and quality-controlled manner in compliance with ethical, legal, and regulatory requirements. This was made possible by a close collaboration between scientists, clinical oncologists, surgeons, pathologists, biobankers, ethicists, and clinical research assistants. From 2014 to 2019 we collected 295 biospecimens from 152 patients enrolled at the three main pediatric centres in Québec, Canada. We faced numerous challenges to offer the study to as many patients as possible. For instance, fresh-frozen tissue samples (tumour resection [72\%], needle biopsy [14\%]), although limited in size, are better suited for molecular profiling, downstream application. For $13 \%$ of cases we had to develop protocols to isolate DNA from tissue samples in formalin-fixed, paraffin-embedded (FFPE) blocks when it was the only source of material. An increasing number of specimens are obtained by needle biopsies, leading to sparse material for diagnosis and potential use for research. We also developed protocols to isolate tumour cells from biopsies and bone marrow with tumour content $<25 \%$ (3\% of cases). In conclusion, biobanking played an important role in our precision medicine program. We demonstrated the feasibility of incorporating genomic sequencing into the management of hard-to-treat childhood and adolescent cancers. The genomicdriven molecular profiling led to the identification of potentially 
therapeutic actionable alterations in most patients tested (66\%). The systematic collection of human samples of high quality is a key element to the success of future treatments.

\section{PD-15 Type 2 Diabetes Pharmacogenomics in Mexico}

H. E. Sánchez-Ibarra ${ }^{1}$, E. A. Valdez-Aparicio ${ }^{1}$, H. A. Barrera-Saldaña ${ }^{2,3}$

${ }^{1}$ Genetics Laboratory, Vitagénesis, Monterrey, Nuevo León, Mexico, ${ }^{2}$ LANSEIDI, Mexico City, Mexico, ${ }^{3}$ Biobank, MexBioBan, Monterrey, Nuevo Leon, Mexico

Background: Type 2 diabetes mellitus (T2DM) is the second most common cause of death in Mexico. This elevated mortality is mainly due to lack of early diagnosis and effective therapeutic control leading to one of every three patients not reaching therapeutic control. The average annual economic cost from 2006 to 2010 to treat T2DM Mexican patients was $\$ 1,146,536,703$ USD of direct and indirect costs. The traditional T2DM prescription approach in which drugs are tested in a trial-and-error manner in each patient until achieving the therapeutic target seems not to help in solving this health crisis.

Methods: In this study, 14 SNPs were screened by qPCR in blood specimens belonging to 495 well clinically described T2DM Mexican patients treated with oral antidiabetic drugs (OADs) from our biobank to find associations between the presence of these polymorphisms, physiological features and response to OADs. Additionally, 100 metformin users' specimens were genotyped using an array for 669,672 genetic variants to find further genotypic associations.

Results: Heterozygous forms of ABCC8-A1369S and KCNJ11-E23K were found to be associated with an increased response to sulfonylureas. Analyzing the metformin users, eight intron variants on transporters SLC47A1, SLC28A1, ABCG2, and SLC7A7 and two variants on ARID5B were associated with reduced levels of $\mathrm{HbA1c}$ by using metformin. In addition to the genotypic data, clinical parameters such as the age of diagnosis, blood pressure, and body mass index were determining factors to reach therapeutic controls.

Conclusions: An individual-oriented management based on patients' phenotype and genotype could help to improve the prescribing and dosing of OADs for T2DM patients, thus increasing the chance to revert this health crisis. These studies were possible thanks to good biobanking practices and well characterization of the specimens involved in the study.

\section{PD-16 Proteomic Signatures of Space Radiation-Induced Cardiovascular Degeneration}

Y. Kidane ${ }^{2}$, F. Lee ${ }^{1}$, M. Bishawi ${ }^{1}$, J. Thompson ${ }^{1}$, A. Moseley ${ }^{1}$, G. Waitt ${ }^{1}$, S. Hines ${ }^{1}$, M. Foster ${ }^{1}$, D. E. Bowles ${ }^{1}$

${ }^{1}$ Duke University, Durham, North Carolina, United States, ${ }^{2}$ Texas Scottish Rite Hospital for Children, Dallas, Texas, United States

Background: We have established a tissue repository of over 700 C57BL6 mice that have undergone radiation exposure at the NASA Radiation Science Laboratory at Brookhaven National Laboratories. Over 12,000 individual biological specimens have been biobanked from these animals. Multiomics study of organs from this biobank of these irradiated mice are expected to inform safe and successful human travel to Mars.

Methods: Quantitative mass spectrometry was performed on peptides (total proteome) as well as phosphopeptides (phosphoproteome) obtained from digested protein homogenates from the hearts of these mice. These mice were subjected to single full-body irradiation at six months of age under the following conditions: 1) $56 \mathrm{Fe}$ ( $50 \mathrm{cGy}$; $\mathrm{n}=3$; obtained three months post-radiation), 2) 160 (50cGy 600 $\mathrm{MeV} / \mathrm{n} ; \mathrm{n}=3$; obtained seven months post-radiation), 3) $56 \mathrm{Fe}$ (50 cGy $1 \mathrm{GeV} / \mathrm{n} ; \mathrm{n}=3$; obtained seven months post-radiation), and 4) Galactic Cosmic Ray (GCR) (150cGy, $n=6$; obtained eight months post-radiation). Bioinformatics analysis of mass spectrometry data sets include: 1) identification of significantly expressed proteins for each dose and radiation types compared to corresponding control samples; 2) pathway enrichment analysis using functional annotation data obtained from Gene Ontology, KEGG (Kyoto Encyclopedia of Genes and Genomes), Reactome, and Molecular Signature Database (MSigDB); and 3) identification of protein and pathway interaction modules that distinguish molecular signature of the various radiation types.

Results: From the hearts of gamma-, 56Fe-, and 16Oirradiated mice, 4,381 proteins and 5,435 phosphopeptides were quantified. Similarly, from hearts from GCR-irradiated mice, 4,650 proteins and 7,002 phosphopeptides (mapping to 303 phosphoproteins) were quantified. While bioinformatics analyses of these data sets is ongoing, several interesting observations are emerging. First, pathways commonly influenced by all radiation types evaluated include mitochondrial gene expression and translation and fatty acid catabolic processes and oxidation. Pathways uniquely modulated by GCR involve cornification and keratinization.

Conclusions: The cornification and keratinization processes observed in heart tissue from GCR-irradiated animals may be contributing to the cardiovascular functional decline observed by hemodynamic and MRI analyses of these mice. Further characterization of the proteins involved in these processes may serve as targets of GCR countermeasures.

\section{PD-17 Effect of Different Brands and Specifications on Sample Melting Time}

T. Huang, Y. Chen, X. Li, X. Zhang, P. Zhang, Y. Zhang

Beijing Friendship Hospital, Capital Medical University, Beijing, China

Background: Cryotubes are the most commonly used consumables in biological libraries, and cryotubes can be used to dispense liquid samples, frozen tissue, and cells. However, with the increasing selection of cryotubes, different brands and different specifications of cryotubes are used in each sample bank. Each time the cryotube containing the sample is taken out of the refrigerator, one needs to wait for it to completely melt before the subsequent test can be carried out. For the time from thawing to complete melting of various types of cryotubes, there is no basis for comparison. One factor is to compare the effects of different brands of different specifications on the complete melting time of the sample.

Methods: A 1-mL sample of 1X PBS phosphate buffer was added to the cryotube to simulate a body fluid sample. The sample was added, the cap was screwed, and it was taken out of the refrigerator after freezing for 15 hours. At room temperature of $24^{\circ} \mathrm{C}$, timing was started and the time of the start of melting and the time of completed melting were recorded. Under the same conditions, three tests were performed and the average was taken.

Results: The melting time was not affected by comparing the wall thickness, the presence or absence of the gasket, and the type of cap. However, by calculating the side area of the 
liquid exposed in the cryotube, it was found that the larger the exposed side area of the cryotube, the faster the melting time.

Conclusions: Exposed side area of cryotube influences sample melting time.

\section{PD-18 Construction of Biological Sample Bank for Clinical Chondrocytes}

J. Li, Y. Zhao, Y. Zhu, X. Zhao, Y. Tian

Laboratory of Translational Medicine, Chinese PLA General Hospital, Beijing, China

Background: There are a large number of cases of cartilage injury that require transplantation in clinic; however, due to easy injury, poor regeneration ability, and difficult access, the clinical application of cartilage tissue is limited. The establishment of a clinical-level chondrocyte bank, to a certain extent, may alleviate the imbalance between clinical operation demand and donor resources.

Methods: The cartilage block was taken from the nonbearing surface of the human joint by operation and single chondrocytes could be obtained after 16-18 hours of digestion using $0.1 \%$ collagenase. Chondrocytes were cultured in chondrocyte culture medium (DMEM/F12 supplemented with $10 \%$ FBS, ITS+, and so on). After reaching $90 \%$ confluence, the cells were passaged by 1:2 and marked as P1. Then the cell characteristics were evaluated and the number and activity of chondrocytes were counted after they reached $90 \%$ confluence again, and at the same time, common bacteria, fungi, mycoplasma, endotoxin, etc., were detected to evaluate the chondrocytes.

Results: A number of primary chondrocytes could be obtained by operation and collagenase digestion. The isolated chondrocytes adhered and grew within 24-36 hours with a polygonal morphology, and generally reached $90 \%$ confluence at 7-9 days. Sufficient P1 cells of approximately 6-8x106 could be obtained after passage by $1: 2$ in $7-8$ days with the cell viability of more than $95 \%$. Test common bacteria, fungi, and mycoplasma were negative, and endotoxin less than $0.125 \mathrm{EU}$. The P1 cells were cryopreserved $(1-2 \times 106 / \mathrm{mL})$ through gradient cooling at $-196^{\circ} \mathrm{C}$.

Conclusion: The establishment of a clinical chondrocyte bank can provide sufficient chondrocytes for tissue-engineered cartilage.

\section{PD-19 Analyte Integrity and Quality Control for Specimens Biobanked using Dried Blood Spot Cards}

\section{G. Lewin, D. Bagar, I. Tarhoni, C. Auger, J. Borgia}

Department of Pathology, Cell and Molecular Medicine, Rush University Medical Center, Chicago, Illinois, United States

Background: Dried blood spot (DBS) card specimen delivery devices offer a convenient and cost-effective alternative to standard phlebotomy for measuring circulating analytes. Currently, DBS is commonly used for genetic neonatal screening and pharmacokinetic studies, but its use for proteinbased assay remains underdeveloped. This platform may offer a convenient and cost-effective means to biobank patient specimens in under-resourced settings with minimal space requirements. This study focuses on the analytical validation of protein biomarker integrity collected via DBS in preparation for an upcoming clinical trial.
Methods: Patient plasma or blood samples from late-stage lung cancer patients were spotted on Whatman 903 filter paper and allowed to dry at room temperature (RT) overnight. Storage conditions were tested by addition of a full-spectrum protease inhibitor cocktail (PI) and stored at RT, $4^{\circ} \mathrm{C}$, and $-80^{\circ} \mathrm{C}$. Samples were extracted at $24 \mathrm{hrs}, 3$ days, 7 days, and 14 days post-storage. Additionally, whole blood samples were spotted at volumes of $10 \mu \mathrm{L}, 50 \mu \mathrm{L}$, and $100 \mu \mathrm{L}$; 6-mm biopsy punches were harvested, with locations (central or peripheral) noted. After extraction, epitope integrity using commercially-available Luminex assays were established.

Results: Recovery results for different storage times ( $24 \mathrm{hrs}$, 3 days, 7 days, 14 days) at $\mathrm{RT}, 4^{\circ} \mathrm{C}$, and $-80^{\circ} \mathrm{C}$ had average MFI values less than 5\% for IGFBP3 with the PI cocktail offering no significant advantage for analyte stability (observed differences less than 5\%). Under the same conditions the PI cocktail offered significant advantages for storage even at $-80^{\circ} \mathrm{C}$ for IGFBP-6 $(\mathrm{p}=0.005)$ and IGFBP-7 $(\mathrm{p}=0.077)$. Central vs. peripheral sections for $100-\mu \mathrm{L}$ plasma was not statistically significant for IL-6 $(p=0.5)$. Spearman correlations of recovered analyte values between spotted blood and plasma specimens were statistically significant across all analytes evaluated (e.g., CD86, CD28, and CD40; p<0.05).

Conclusion: The DBS specimen delivery method offers a stable platform for collecting specimens that provides comparable analytical results relative to conventional phlebotomy. Analytes were stable under the tested storage conditions with protease inhibitors offering significant advantages. Further testing is currently underway using additional patient specimens and multiple card types with several distinct classes of proteins (immunoglobulins, soluble receptor proteins, and globular proteins) being evaluated.

\section{PD-20 Method Optimisation for Isolation and Culture of Human Adipocytes}

C. Bellora, F. Betsou, O. Kofanova

IBBL (Integrated BioBank of Luxembourg),

Dudelange, Luxembourg

Statement of the Problem. Adipogenic cell systems are increasingly relevant for biomedical research and development in the field of obesity and diabetes. Differentiated human adipocytes are used as a screening tool by pharmaceutical industries. However, there are some limitations such as a long differentiation protocol, large donor-to-donor variation, and associated significantly high costs. Biobanking of an optimized adipogenic cell systems, which respond adequately to the functional assays, shows a partnership and perspectives for those collaboration purposes. For example, cryopreservation of primary pre-adipocytes provides the possibility to overcome the limitations, but requires skilled handling, especially regarding the isolation and later differentiation steps, as well as the preparation of the cell bulk for storage.

Proposed Solution. We have developed and optimized a short and robust workflow from isolation and cultivation to differentiation and cryopreservation of human primary pre-adipocytes. The primary pre-adipocytes were isolated from the stromal vascular fraction of human abdominal lipoaspirates. Several kits have been tested in order to establish a robust process for cell culture incubation, differentiation, and characterisation. We have also implemented immunoenzymatic QC assays to qualify the function of the cell culture by measureing adiponectin and leptin levels.

Conclusions. The developed and optimized protocol will allow us to launch large-volume cell cultures, produce ready-to- 
use cryopreserved pre-adipocytes of standardized quality, and, furthermore, additionally develop customised functional QC assays.

\section{PD-21 Establishment of an Induced Pluripotent Stem Cell Line from a Patient With Long QT Syndrome}

H. Zhou

Biobank, East Hospital Affiliated to Tongji University/Shanghai East Hospital, Shanghai, China

Long QT syndrome type 8 is an uncommon inherited condition. An induced pluripotent stem cell (iPSC) line was generated from peripheral blood mononuclear cells (PBMCs) of a 10 -year-old patient with heterozygous mutation of p.R858H (c. $2573 \mathrm{G}>\mathrm{A}$ ) in the CACNA1C gene. This iPSC model offers a very valuable resource to study the disease pathophysiology and to develop therapeutics for treatment of Long QT syndrome type 8 patients.

Resource utility: The iPSC line is derived from PBMCs from a 10-year-old patient with Long QT syndrome type 8 . This iPSC line will be useful for disease modelling and help to better understand the disease-causing mechanism, which would enable development of new treatments for LQT 8. It may provide a cellular model for exploring the pathogenesis, screening drugs, and developing new therapies.

\section{PD-22 Fitness-for-Purpose of Stabilized Stool Samples for Specific Metabolite Analyses}

L. Neuberger-Castillo, W. Ammerlaan, F. Betsou

Integrated BioBank of Luxembourg (IBBL),

Dudelange, Luxembourg

Background: Biobanks and large-cohort studies are increasingly utilizing OMNIgene ${ }^{\circledR}$-GUT kit to collect and store stool samples for downstream DNA-based microbiome analyses. While the kit permitted ambient-temperature collection and storage of samples for gut microbiota studies, the use of the same sample type for downstream metabolomics assay has not been explored. Microbiome-metabolomics analysis of fecal samples is increasingly getting attention to further elucidate the mechanisms by which the gut microbiota influences the host. Therefore, in this study, we evaluated the fitness-for-purpose of the OMNIgene ${ }^{\circledR}$-GUT-collected stool samples for downstream metabolomics assay in the scope of the fecal bile acids (BA) quantification.

Methods: Biocrates ${ }^{\circledR}$ Bile Acids Kit was used for the quantification of BA from eight healthy donors' samples collected in 1) OMNIgene ${ }^{\circledR}$-GUT kit and 2) snap frozen in $-80^{\circ} \mathrm{C}$ in duplicates. A highly selective reversed-phase LC-MS/MS analysis method in negative ion multiple reaction monitoring detection mode was applied to determine the BA concentrations in each sample.

Results: We found that the total fecal BA levels are detectable in OMNIgene ${ }^{\circledR}$-GUT-collected samples, with a mean of $323.8 \mathrm{pmol} / \mathrm{mg}$ and with a range of $29.9-903.7 \mathrm{pmol} / \mathrm{mg}$. Overall, extractions from snap-frozen samples resulted in higher concentrations of total BAs (mean: $578.1 \mathrm{pmol} / \mathrm{mg}$; range: $243.7-$ $1136.2 \mathrm{pmol} / \mathrm{mg}$ ). This can be attributed to the fact that the OMNIgene ${ }^{\circledR}$-GUT-collected samples are suspended in stabilizer (approximately $500 \mathrm{mg}$ of sample in $1.5-\mathrm{mL}$ stabilizing liquid), resulting in lower total fecal BA concentrations. Despite this, we found no significant difference in the proportion of primary BAs to the total BA concentrations in OMNIgene ${ }^{\circledR}$-GUT-collected samples as compared with the snap-frozen samples $(\mathrm{p}>0.05)$. The same result is seen with the proportion of secondary BAs to the total BA concentrations in both sample collection methods.

Conclusions: We conclude that the OMNIgene ${ }^{\circledR}$-GUTcollected stool samples are fit-for-purpose for downstream metabolomics assay in the scope of the fecal BA quantification. This suggests that more than one stool collection procedures are suitable for metabolite analyses. However, the reference ranges of the total and individual fecal BAs are different in OMNIgene ${ }^{\circledR}$ GUT-collected samples and in snap-frozen samples. Thus, establishment and maintenance of a standard stool collection procedure is critical.

\section{PD-23 Post-operative Delirium Associated with Metabolic Alterations Following Hemi-arthroplasty in Older Patients}

Y. Zhang, H. Lu, W. Jia

Shanghai 6th People's Hospital, Shanghai, China

Background: Post-operative delirium (POD) is a common complication in older patients, though a possible link between metabolic changes and POD development has yet to be investigated.

Methods: Older patients with hip fracture who underwent hemi-arthroplasty were recruited, and delirious states were assessed for three days after surgery using the confusion assessment method-Chinese revision. Simultaneously, fasting blood samples were collected on the morning of surgery and on the first post-operative day. Ultimately, 244 older patients who met the inclusion and exclusion criteria were assessed. Blood samples from 60 patients with POD and 60 matched controls were analysed using metabolomics platforms.

Results: Sixty patients (24.6\%) developed POD. Principal component analysis scores plot and cross-validated scores plots from orthogonal partial least squares-discriminant analysis were implemented to visualise the differences in metabolites between the two groups before and after surgery $(\mathrm{P}<0.05)$. Our data indicate that levels of $\omega 3$ and $\omega 6$ fatty acids were lower in the POD group than in the NPOD (non-POD) group both before and after surgery; tricarboxylic cycle intermediate levels were lower in the POD group than in the NPOD group, but glycolysis products were higher in the POD group than in the NPOD group after surgery. Furthermore, the branched-chain amino acid (BCAA)/aromatic amino acid ratio was lower in the POD group than in the NPOD group after surgery.

Conclusions: Metabolic abnormalities, including deficiencies in $\omega 3$ and $\omega 6$ fatty acids, perturbations in tricarboxylic cycle, and oxidative stress and metabolic imbalances in BCAA and AAA, might contribute to POD development.

\section{PD-24 Banking of Tumor Tissues: The Minimal Effect of Ex Vivo Ischemia Time, Freeze-thaw Cycles, and Cryopreservation Time on RNA Integrity?}

X. Zheng, W. Jia

Biobank, Sun Yat-Sen Univeristy Cancer Center, Guangdong, China

Background: Tissue bank has become a major initiative at many cancer centers. Pre-analytical variables have significant effects on RNA integrity in tumor tissues, leading to bias in 
gene expression analysis. However, the effects of ex vivo ischemia time, freeze-thaw cycles, and cryopreservation time, three of the most common problems in a tissue bank, have not been well understood.

Methods: Four hundred sixty-six cases of tissues with tumor cell content $\geq 75 \%$ out of 896 cases from 12 common types of tumor were first selected to measure the RNA integrity under a uniform protocol, in order to choose the tumor tissue with the best tolerances to pre-analytical variables. One hundred four cases of tumor tissues from liver were further collected in the course study, in order to evaluate the minimal effect of ex vivo ischemia time, freeze-thaw cycles, and cryopreservation time.

Results: Tissues from liver had the highest percent $(69.7 \%)$ of samples with tumor cell content $\geq 75 \%$ and had the best RNA integrity (mean \pm standard deviation $=8.4 \pm 0.73$ ). A tendency toward lower RNA integrity number (RIN) was observed both in time and freeze-thaw course study. Prolonged ex vivo ischemia time, freeze-thaw cycles, and cryopreservation time decreased the RIN at a rate of approximate $0.195,0.147$, and 0.274 units per time, respectively.

Conclusions: RNA integrity of tumor tissues shows tissue type dependence and tumor tissue from liver has better tolerance than the other 11 tumor types. It suggests collection protocols may be adjusted for a specific tissue type. According to the effects of these three variables, a preliminary estimation of RNA integrity in tumor tissues could be conducted. Our study provides some valuable data for developing evidence-based standard operating procedures.

\section{PD-25 Preserving Unique Genetic Resources of Nguni Cattle for Sustainable Production in Changing Environments}

\section{A. A. Zwane ${ }^{1}$, M. Ramothwala ${ }^{2}$, K. Nxumalo ${ }^{1}$ \\ ${ }^{1}$ Animal Breeding and Genetics, Agricultural Research Council, Pretoria, Gauteng, South Africa, ${ }^{2}$ Tshwane University of Technology, Pretoria, South Africa}

The success of genome-wide association studies in humans has spurred interest in the use of large-scale, high-density SNPs genotyping for the detection of genomic variation affecting complex traits, identification of QTLs, and marker-assisted selection in livestock species. Indigenous livestock have important functions of provision of food and income to socio-economic, cultural and ecological roles. They also have adaptive traits ranging from drought tolerance, resistance to ticks and tick-borne diseases, heat tolerance, and resistance to trypanosomosis, traits needed for changing environments. Climate change, on the other hand, impacts on the amount and quality of produce, reliability of production, and the natural resource base on which production depends. Indigenous cattle breeds of South Africa, Afrikaner, Bonsmara, Drakensberger, Nguni, and Tuli, provide a valuable genetic resource that supports range of diverse human communities with reasonable products such as meat, milk, and leather, as well as various socio-cultural functions. These breeds have good adaptive traits and provide reliable resources for climate changes. Genomic improvement on these breeds is very important in maintaining their diversity and sustainable production for food security. Recent genome-wide studies on Nguni cattle have detected positive selection using SNP arrays and sequence data; however, there is lack of information regarding the gene functions underlying adaptive and production traits on this breed. Gene annotation and enrichment analysis of the identified selective sweeps in Nguni cattle identified about 58 genes that were associated to eight randomly chosen phenotypes queries (fat, milk production, walking ability, heat tolerance, meat production, reproduction, and bone and muscle development). The fold enrichment was used to select only the top-listed genes associated with phenotypic characteristics. Gene ontology identified NOD2 and IL21R genes that were associated with inflammatory bowel diseases in cattle. These genes serve as useful genetic tools for genetic variation in cattle improvement.

\section{PD-26 A Washing Method to Improve the Quality of RNAs Isolated from the "Buffy Coat" of Blood Samples}

\section{Y. Chen, H. Lu, R. Zhou, M. Liang, S. Deng \\ Biobank of Southwest Hospital, Army Medical University, Chongqing, China}

In the process of RNA isolation, one of the services of our biobank, we found that it is very hard to get high-quality RNAs from the buffy-coat blood samples by conventional isolation and wash methods, such as Trizol reagent extraction. The A260/A230 ratio was always low (less than the standard of 1.80), which did not meet the quality requirements. It is known that the low value of A260/A230 indicates salt ion contamination, which may affect subsequent experimental results, such as gene expression detection and deep sequencing, etc. In order to reduce the concentration of salt ions to enable RNA samples to meet the quality requirements, we further explore the washing methods in RNA extraction (by using Trizol reagent) from the buffy-coat blood samples. We found that the A260/A230 ratio got by two times wash with $70 \%$ ethanol significantly increased from $0.87 \pm 0.27$ (by one wash) to the value of $1.47 \pm 0.38$, but still less than 1.80 . The value got by three times wash (with the value of $1.42 \pm 0.62$ ) did not increase any further compared with two times wash. However, the A260/A230 ratio processed by a three times wash with an additional $16-24 \mathrm{~h}$ placing in $4{ }^{\circ} \mathrm{C}$ increased significantly with the value of $2.05 \pm 0.29$, which meets the quality requirements. A longer placing time $(72 \mathrm{~h})$ would reach the A260/A230 value of $2.02 \pm 0.2$, which was not significantly different from the placement of $16-24 \mathrm{~h}$. We also found that the increase of the number and time of washing had no significant effect on another reference, A260/A280, of RNA quality. In addition, we also used two commercial RNA extraction kits (non-Trizol type) for RNA isolation of buffy-coat blood samples, and found that the RNA quality from kit $\mathrm{A}$ is low; both the value of A260/A230 $(0.79 \pm 0.3)$ and A260/A280 (1.68 \pm 0.17$)$ did not meet the standard. Kit B has a good effect, with the A260/A230 value of $1.93 \pm 0.37$, and the A260/A280 value of $1.82 \pm 0.27$, in line with the quality requirements. However, the RNA yields from buffy coat per milliliter blood sample of kit A and kit B were much lower than those from the Trizol method: Kit A $(399 \pm 206 \mathrm{ng})$,

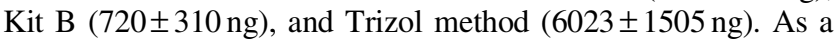
result, Trizol approach, in conjunction with the improved washing methods, provides a unique advantage for the biobank to provide researchers with sufficient and quality-compliant RNAs from buffy-coat blood samples.

\section{PD-27 Evolution of Cancer-Related Biospecimen Needs by Scientists from Industry}

\section{B. Britsch, H. Lahlou, P. Puchois, L. Stephan}

Trans-Hit Biomarkers, Laval, Québec, Canada

Background: Tissue samples are among the most requested biospecimens by scientists from industry in order to support their cancer biomarker R\&D projects. 
Methods: We have assessed the type of cancer-related biospecimen requests Trans-Hit Biomarkers has received since 2015 and analyzed how the requests evolved over the years, with a focus on requests for tissue biospecimens.

Results: Trans-Hit Bio has received 1,244 requests for cancer biospecimens in five years (2015 to 2019); 68\% of them concerned tumor tissues. On average, $92 \%$ of these requested were for FFPE tissues and $8 \%$ for frozen tissues. The ratio frozen to FFPE has changed over the years, with $14 \%$ of tissue requests being for frozen tumor tissues in 2015, decreasing to $5 \%$ in 2019.

While all the requests in $2015(n=145)$ were for either frozen or FFPE tissues, we then started to receive an increasing number of requests for prospective collections of freshly resected viable tumor tissues, culminating in a peak of $12 \%$ in 2018 $(\mathrm{n}=23$ for fresh vs. $\mathrm{n}=169$ for non-fresh).

A similar trend was observed concerning the requests received for matched cancer samples (e.g., at least two different types of biospecimens collected from the same patients). Although none were seen in $2015(\mathrm{n}=202)$, they represented $14 \%$ (of $n=292$ ) in 2018 and 12\% (of $n=294$ ) in 2019. Moreover, on average, since $2015,63 \%$ of the matched cancer requests were for FFPE tissue samples matched with another type of biospecimen (e.g., plasma).

Conclusion: Our analysis shows that industry scientists need FFPE tumor tissue samples more than any other type of cancer biospecimen. Snap-frozen tumor tissues are less and less demanded and used, probably due to the development of technics allowing to isolate total nucleic acids from FFPE tissue samples. Despite important investments carried out during the last 20 years to collect and store frozen tumor tissue samples, industrial scientists demand less of them, in contrast to FFPE tissue samples, which, ideally, will have to be well-annotated with associated clinical data including medical follow-up. Moreover, requests for plasma matched with FFPE tissue samples, and for freshly resected tumor tissues, are becoming more frequent. They correlate with IVD and pharma companies' increasing interests in the development of liquid biopsies assays and immunotherapies, respectively.

\section{PD-28 Effects of Primary Non-Conformances on the Quality Control Metrics in a Large Biorepository}

K. H. Driesbaugh, D. M. Kusic, G. Smith, S. I. Wert, N. Turan

Coriell Institute for Medical Research, Camden, New Jersey, United States

Biobanks play a pivotal role in enabling biomedical research and increasing our understanding of genetics and genomics in critical disease pathways. The significance and relevance of a biobank collection relies on both the quality of the submitted biospecimens and the processed research material utilized for scientific examination. It is imperative that quality control (QC) parameters be considered for the primary biological specimen and the processed research material alike. Using data collected from a large biorepository, we analyzed the QC metrics of genomic DNA processed from $\mathrm{N}=30,061$ unique whole blood and saliva samples collected from adults and children, including DNA purity (260/280, 260/230 ratios), and DNA integrity number (DIN). Sample submission non-conformances were predefined at collection set-up as factors that may impact the quality or integrity of the sample, including 1) days from sample collection to processing, 2) sample storage and shipment temperature, and 3) the deviation from the expected sample fill volume. Over $95 \%$ of all sample submissions passed all successful QC parameters (2\% DNA from whole blood, $17 \%$ DNA from saliva). Importantly, DNA extracted from whole blood processed greater than four days post-collection show a failure rate of more than $9 \%$ compared to $1 \%$ failure rate from samples extracted four or fewer days post-collection, demonstrating the importance of timely sample processing. Whole blood shipped for processing during the warmer summer months of June, July, and August show a failure rate of more than $3 \%$ compared to a failure rate of less than $2 \%$ for whole blood shipped for processing during the cooler months of fall, winter, and spring. Similarly, the accuracy of blood tube fill volume is also critical to processed DNA quality. Blood tubes submitted with more than $3 \mathrm{~mL}$ underfill from the manufacturer recommendation demonstrated a failure rate of $5 \%$ compared to those that were within $3 \mathrm{~mL}$ of the fill volume with less than a $2 \%$ failure rate. A similar trend is seen with saliva sample submissions. These data demonstrate that deviation from ideal sample collection and transport has measurable impact on quality control metrics that may ultimately affect downstream research applications using precious biospecimens.

\section{PD-29 An Automatic Cryopreservation System Inhibits Temperature Fluctuation and Reverses the Reduction of Cell Viability and Functional Activities of Cryopreserved Cells}

Y. Xu

Shanghai Origincell Medical Technology Co. Ltd, Shanghai, China

During long-time cell storage, a major challenge is the temperature fluctuation caused by the explosion of cryopreserved cells to room temperature. Consequently, the viability, recovery and functionality of cells are seriously impaired. Here we focused on the influence of temperature fluctuation on the three most important cell types for cell-based therapies: peripheral blood mononuclear cells (PBMCs), umbilical cord-derived mesenchymal stem cells (UC-MSCs), and chimeric antigen receptor T cells (CAR-T cells). An automatic robotic device is designed to simulate storage conditions including sample storage, removal, and sorting in large biorepositories. Cryopreserved cell samples were exposed from the liquid nitrogen temperature to the room temperature until the sample temperature rise to $-60^{\circ} \mathrm{C}$ and then put it back into liquid nitrogen temperature for 0 up to 400 cycles. In parallel, the same cell samples were conducted and transferred within fully automatic cryopreservation system ensuring a whole cold chain with a temperature under $-150^{\circ} \mathrm{C}$ for the comparable cycles. Our data showed that the fully automatic system protected the samples from the influence of temperature fluctuation and enabled high viability and proliferation activities of PBMCs, MSCs, and CAR-T cells during the cycles of sampling process. For cells in automatic robotic device: the cell viability and functional activities of PBMCs were significantly decreased with the increase of the sampling process cycles. Notably, 200 sampling process cycles did not affect the viability and recovery of PBMCs, but they had a significant influence on the proportion, proliferation, and PHA immune response of PBMCs subsets. The viability, recovery, proliferation, and differentiation capacity of UC-MSCs were all significantly decreased with the increase of the sampling process cycles. The cytotoxic activity and immune response of CAR-T cells were also significantly decreased by temperature fluctuation. The fully automatic system further ensured the stability of PBMCs phenotype, the multi-lineage differentiation capacity of MSC, and the cytotoxic activity of CAR-T cells, indicating that 
the fully automatic system may be a solution for avoiding the influence of temperature fluctuation during the development of a biobank in a large scale.

\section{PD-30 Investigating Solution Effects Injury of Human T Lymphocytes during Interrupted Slow Cooling using Ice Seeding}

W. Liu

University of Shanghai for Science and Technology, Shanghai, China

The cooling rate is a critical parameter for cell cryopreservation which directly affects the success of cell storage. Fast cooling can result in intracellular ice formation, while slow cooling could bring solution effect. However, the solution effects injury during slow cooling has received little attention thus far. In this paper, the damage of human $\mathrm{T}$ lymphocytes during slow cooling was studied by cryomicroscope and interrupted slow cooling. We found the cell damage during interrupted slow cooling was caused by the high osmotic pressure rather than the formation of eutectic phase. We further tested the osmoprotective ability of some frequently used cryoprotectants (CPAs) including dimethyl sulfoxide (DMSO), glycerol, trehalose, urea, and L-proline. Interestingly, we found that DMSO, glycerol, and trehalose can protect cells from osmotic damage during slow cooling. Trehalose had the best osmoprotective ability compared with other studied CPAs. However, L-proline and urea lacked the osmoprotective ability. Finally, we studied the relationship between the degrees of cell volume change and the cell damage and found that cell volume change is not a key factor for osmoprotective ability of CPAs. This study confirms that the slow cooling cell injury is caused by high osmotic pressure rather eutectic formation by interrupted slow cooling and suggest that cell volume may not be a key factor in explaining solution effects injury for cells and osmoprotective ability for CPAs.

\section{PD-31 The Impact of Automation and Addition of Proactive Quality Checkpoints on the Quality of DNA at Qatar Biobank}

S. Alresheq, E. Alkhayat, A. AlAnsari, T. Alhamad

Laboratory, Qatar Biobank, Doha, Qatar

Background: Having established its pioneer role in raising Qatar's profile of biomedical research, Qatar Biobank seeks to constantly increase the efficiency of its performance. One of the vital roles Qatar Biobank plays in the field of research is to provide DNA samples for different research projects. Initially, the source of DNA was buffy coat extracted manually from whole blood EDTA tubes. In order to improve DNA quality, shifting from the process of extracting buffy coat manually without applying any pre-analytical checkpoints to the process of automating buffy-coat extraction and adding proactive checkpoints was implemented. In this study, the impact of shifting from one process of buffy-coat preparation to another on DNA quality is presented.

Methods: DNA was extracted from 1,000 buffy-coat samples. Of which, 500 buffy-coat samples were extracted from EDTA tubes by (Process-A: manually, without proactive checks). The other 500 samples were extracted from EDTA tubes by (Process-B: automation, with proactive checks on both EDTA tubes and buffy-coat tubes). Concentrations and yields were measured for DNA samples using photometric and fluorometric methods to assess the modifications implemented on the process.

Results: The average concentration and the average yield were significantly higher for samples treated with Process-B (automated with proactive checkpoints) than for Process-A (manual without proactive checkpoints), with an increase of $300.2 \%$ for yield and $157.14 \%$ for concentration.

Conclusions: Shifting the process of buffy-coat extraction from manual without proactive checks to automation with proactive checks has significantly improved the quality of DNA output at Qatar Biobank.

\section{PD-32 Surface Marker Characterization of Primary Derived Human Umbilical Vein Endothelial Cells Prior to Biobanking}

R. Follett ${ }^{1}$, H. Monforte ${ }^{1,5}$, J. Cross $^{1}$, D. Skaar ${ }^{2}$, S. Johnson ${ }^{3,4}$, W. Schleif ${ }^{1,5}$

${ }^{1}$ Johns Hopkins All Children's Pediatric Biorepository, Johns Hopkins All Children's Hospital, St. Petersburg, Florida, United States, ${ }^{2}$ Department of Biological Sciences, North Carolina State University, Raleigh, North Carolina, United States, ${ }^{3}$ Department of Pediatrics, Johns Hopkins University School of Medicine, Baltimore, Maryland, United States, ${ }^{4}$ Department of Population, Family, and Reproductive Health, Johns Hopkins University, Baltimore, Maryland, United States, ${ }^{5}$ Program in Pediatric Biospecimen Science, Johns Hopkins All Children's Institute for Clinical and Translational Research, St. Petersburg, Florida, United States

Statement of Problem: Isolation of human umbilical vein endothelial cells (HUVEC) directly from cord tissue is commonly done to construct primary cell lines. The efficiency and consistency of this process is typically not assessed immediately, as most protocols utilize a simple cobblestone cell phenotype to ascertain cell type after several days have lapsed in culture. Deeper characterizations are typically performed post-culture without accounting for in vitro induced variation that may misrepresent the original sample. Epigenetic characterization of HUVECs requires a different approach, as the presence of different cell fractions derived during the enzymatic digestion and culture bias will complicate the profiling of HUVEC methylation patterns. This problem could be more pronounced in larger prospective banking cohorts involving multiple institutions with different procurement staff, enzyme lot-to-lot activity variation, and protocol adherence.

Proposed Solution: We propose a pilot multi-institutional method to prospectively isolate and bank HUVEC DNA, characterized for uniformity using a small subset of fixed cells $(<10 \%$ of the total isolated fraction) stained with dual immunofluorescence cell surface markers (CD31, CD144) over a two-year period as support for a large birth cohort study. Antibody specificity is confirmed using fixed mount slides and digital microscopy. The efficiency of the collection is assessed using immunohistochemistry to investigate pre- and post-enzymatic digestion depths and total fraction cell counts using AO/PI image cytometry.

Conclusion: This model provides a number of key indicators useful in monitoring the quality of primary derived HUVEC cell fractions banked for downstream analysis. These indicators are particularly useful in large, multicenter biobanking collections as an assessment tool where methodological variations are likely to occur and are deemed detrimental to sensitive analytical approaches like epigenetic characterizations that may be misinformed if the target cell population of interest is contaminated with mixed cell subsets. 


\section{Commercial Biobanking}

\section{PE-01 Stem Cell Storage as a Framework for Biobank Sustainability}

\section{S. Gramatiuk}

\section{Ukraine Association of Biobank, Kharkiv, Ukraine}

Quality biological samples stored in biobanks are essential for the provision of appropriate health services and also act as a reservoir for the development of precision medicine and biotechnological innovation. The Ukrainian Association of Biobanks (UAB) coordinates the network procedures, based on ESBB, ISBER, BBMRI, and NCI guidelines, which are standardized across the entire network. Cell-based regenerative medicine holds significant medical opportunity, but we have also seen some bad physician leverage, the scientific promise of this field to peddle unapproved treatments that put patients' health at risk.

The Ireland pathway was identified as the most effective for Ukrainian local circumstances and it was decided to adopt a similar sequence of document development and submission. First, the consensus was reached with each hospital within the network. Second, a review of documents by the Risks and Legal Department in $\mathrm{UAB}$ was performed. In parallel a formal legal statement was obtained from an independent lawyer specialized in medical research.

The first project phase successfully established routine and robust procedures for collecting, banking, characterising, and distributing stem cell lines. The collaboration of iPSC and MSC research projects and deposit of established, authentic, stem cell lines made from normal consenting donors were pivotal in validating many of these standard procedures.

The Ukraine Association of Biobank has begun a clinical study on the treatment of post-viral fibrosis and liver cirrhosis using mesenchymal stem cells.

Conclusion: The UAB short- to medium-term objectives include expanding the current network and to include each of the major medical research centers in Ukraine. Enlargement of the network could be undertaken with modest capital investment and UAB medium size will facilitate a swift and dynamic approach to network expansion. By now, the UAB is reaching its goal: to develop infrastructure and facilitate the biomedical research nationally and internationally.

\section{PE-02 Steps towards Fiscal Sustainability - Structuring Research Staffing Under a Professional Staffing Model in a Health System-Based Biorepository}

S. D. Firth, C. Sainsbury, B. Sharp, S. Stradling,

M. H. Cessna, D. K. Crockett

Biorepository, Intermountain Healthcare, Salt Lake City, Utah, United States

Background: Intermountain Healthcare's Biorepository collaborates with commercial, academic, and internal research partners. Intermountain's executive leadership have continued to invest in the modernization and expansion of the Biorepository. Financial self-sufficiency among its research services team will contribute to the longevity of the repository. Working towards fiscal sustainability will ensure that Intermountain's Biorepository is positioned to provide biospecimens to key research partners in their efforts to advance the future of diagnostics and treatment of disease.
Methods: Intermountain Healthcare's Biorepository research team is structured into two teams with prospective and retrospective foci. Both are led by research managers, and staffed by research coordinators (RC) and research assistants (RA). These teams collaborate with investigators/sponsors to obtain qualifying study materials and relevant patient data for their study aims. The Biorepository is exploring the feasibility of a professional staffing services model to achieve two ends: 1) ensure adequate staff levels to support current and pipeline projects while maintaining engaging and meaningful work to prevent burn-out, and 2) provide enough effort bandwidth to staff new studies or consented banking-only protocols.

Results: The Biorepository utilizes a comprehensive timetracking tool, enabling project specific time tracking, with taskcategory specificity, allowing RCs and RAs to track their time in detail. Overlaying time-tracking data over RC and RA fully burdened rates provides cost data on a project and task level. Comparing cost data performance against completed project invoices yields metrics on how percent of effort performs against the Biorepository's current price model and identifies opportunities to improve processes and deploy tools that optimize workflow. An idealized goal is to have RCs, and RAs, fully burdened rates covered by billable activities at about a $50-60 \%$ level of effort.

Conclusions: Improving and optimizing processes and aligning the Biorepository's price model to attain a level of effort of $50-60 \%$ that is completely billable and covers fully burdened costs leaves $40-50 \%$ effort free for sustainable growth and alternative growth-oriented activities (banking-only protocols). The bandwidth remaining can be used to expand to new projects and protocols, allowing for the hiring and onboarding of new RCs and RAs to assume those efforts, and do so fully billable.

\section{Ethical, Legal, and Social Issues}

\section{PF-01 Consent, the Pivot in Research in Ghana; Breast Care International Best Approach}

\section{E. Mensah ${ }^{1,2}$}

${ }^{1}$ IT / Biobank / Research, Breast Care International, Kumasi, Ghana, ${ }^{2}$ Information Technology, Peace and Love Hospital, Kumasi, N/A, Ghana

Background: Breast cancer is becoming a major public health challenge in Africa and most countries do not have cancer registries; nevertheless, the World Health Organisation estimates that the incidence rate of breast cancer in Africa has steadily increased over the years. According to 2018 Globocan report, Ghana recorded 4,645 (20.4\%) new breast cancer cases. Although it is acknowledged that early detection reduces mortality, $80 \%$ of newly diagnosed breast cancer patients delay presentation. Early detection and prompt treatment have been the focus of Breast Care International (BCI). Since its inception, in 2002, BCI has developed a well-structured outreach program that reaches a significant portion of the population with the goal of receiving the consents of the masses to enhance research and other projects such as biobanking.

Objective: To explore the effectiveness of informed consent that promotes education, treatment, and research in breast cancer for further evaluation and treatment.

Methods: Using the Ghana Breast Health Study as a case study, BCI collaborates with opinion leaders, organized groups, and local community to explain the project purpose and importance; role of the individual, stakeholders, and community; and increase their knowledge on consent. A consent form is provided by the sponsor with the study protocol or created by the 
site investigator. The consent form is personalized by each site, adding local contact names and numbers. The consent is approved for use by Institutional Review Board. The investigator or designated study personnel informs the participant about the study purpose, risks, and potential benefits. The patient is allowed time to read the consent form, ask questions, and consider participation. Participant or representative signs and dates the consent form. The sponsor and/or Institutional Review Board may require additional signatures. The participant is given a copy of the consent and taken to the phlebotomy unit for sampling.

Results: The BCI method has improved the knowledge, attitudes, and involvement of individual, organized group, and opinion leaders regarding consent for a project.

Conclusion: A short community-based education on consent might influence huge participation in a project in Ghana.

\section{PF-02 Willingness of Participants to Donate Samples and Data for Biomedical Research in Collaborations between Academic Biobanks and Industry}

\section{Specht, C. Klingler, M. L. Hartung}

\section{German Biobank Node (GBN), Charité-Universitätsmedizin} Berlin, Berlin, Germany

More than three quarters of Germans are willing to make their health data available anonymously and free of charge digitally for medical research. This is the result of a representative FORSA survey of 793 participants from September 2019. At the same time, the willingness to support academic research is extremely high, whereas only 17 percent want to support research conducted by private companies and industry.

The German Biobank Node (GBN) as an umbrella organization for academic biobanks investigates the question of what impact collaboration of biobanks with industry partners could have on the willingness of participants to donate biosamples and data. Therefore, GBN actively seeks the dialogue with participants and representatives of patient organizations in order to discuss the opportunities and obstacles of biomedical academic research in cooperation with industry. The aim is to understand the reservations and concerns and to jointly define concrete measures to enable patients to make an informed and self-determined decision as to whether they agree to donate samples and data.

Will patients possibly withdraw their consent, if they learn who has received their biosamples and/or data even though they have agreed by broad consent to the possibility for private companies to use their samples/data? Does collaboration with industry have a positive or negative impact on patient confidence in academic biobanks? Could this reduce the willingness of patients/study participants to donate? These and other questions are discussed with patient representatives during nonpublic focus group workshops. The aim is to identify ethical implications of collaboration between academic biobanks and industry from the donor perspective. The attitudes of patient representatives with different disease backgrounds and potential effects on their willingness to donate samples and data are the primary focus but also the difference between sample and data donation and the resulting ethical implications are discussed.

The goal is to formulate a framework for cooperation between biobanks and industry, under which patient representatives would be prepared to donate their own samples and data. Another goal of the focus groups is to develop a concept for an ELSI workshop on the topic of "Willingness to donate samples and data for research in cooperation between academic biobanks and industry" with representatives from industry, biobanks, ethicists, lawyers, and patient representatives.
PF-04 Biospecimen Donation in Biobank Construction: Aspects Affecting Donation and Public's Concern

\author{
Z. Zhou ${ }^{1}$, H. Yu ${ }^{1}$, Y. Zhang ${ }^{1}$, B. Qin ${ }^{1}$, J. Huang ${ }^{2}$
}

${ }^{1}$ Department of Anatomy and Neurobiology, Central South University, Changsha, China, ${ }^{2}$ School of Life Sciences, Central South University, Changsha, Hunan, China

Background: A biobank is a storage facility which stores samples mainly for biological or medical researches. Biospecimen collection is the kind of work essential in the building of a biobank. However, we found the collection works had difficulties in the proceeding because of the low participation rate.

Methods: We conducted a questionnaire including several questions covering several aspects in order to find out people's attitude towards biospecimen donation. The questionnaire had 20 questions mainly focused on overall public participation rates, matters that influence their participation, and major public concerns in biospecimen donation.

Results: In our survey, responses were received for 1,477 from 2,200 distributed questionnaires, and electronic questionnaires showed the highest response rate of $49.9 \%$. In all respondents, 936 showed willingness in participation, providing a percentage of $63.4 \%$. We found that most respondents lack the knowledge of biospecimen donation and biobanking but still have a positive attitude towards biospecimen donation. Several factors, including family disease history $(\mathrm{p}<0.05)$, previous donation history $(\mathrm{p}<0.01)$, the knowledge of biospecimen donation $(p<0.01)$, and the knowledge of biospecimen donation conception $(\mathrm{p}<0.01)$, were closely linked to donation willingness. Among those factors, family disease history and brief knowledge about biospecimen donation were independent factors affecting donation willingness. The reverse health effects and privacy leakage were major concerns among the majority of respondents. Primary reasons affecting willingness, or unwillingness, to donate were their benefit to public interests and privacy concerns. In summary, in this survey we mainly discussed factors affecting the public's willingness and issues people concerned most in biospecimen donation of a biobank.

Conclusions: Most respondents hold positive attitudes towards biospecimen donation but lack relevant knowledge. Several factors influenced donation willingness, probably caused by those deficiency knowledge. Even though faced with this challenge, responders' merits of altruistic behavior may contribute to the act of donation. In addition, information leakage and health impairment remained the domain factors prevented their participation. Further works are required to eliminate those undesirable elements that restrained biospecimen donation by the well biobank knowledge popularization and detailed pre-donation information exchange.

\section{PF-05 Clinician Engagement in Biobanking - A Melbourne Health Tissue Bank Perspective}

\author{
Z. Zhao ${ }^{1,2}$, L. Taylor ${ }^{1,2}$, K. Velasco ${ }^{1,2}$, W. $\mathrm{Ng}^{1}$ \\ ${ }^{1}$ Victoria Cancer Biobank, Melbourne, Victoria, Australia, \\ ${ }^{2}$ Melbourne Health Tissue Bank, The Royal Melbourne \\ Hospital, Melbourne, Victoria, Australia
}

Statement of problem: The Victorian Cancer Biobank was established with support from the Victorian Government to provide high-quality clinically annotated biospecimens to the academic and commercial cancer research sectors. Members of this consortium are embedded within major Victorian Hospitals, with the Melbourne Health (MH) Tissue Bank being located at the Royal Melbourne Hospital (RMH). Attainment of informed 
consent is the key initial step to obtaining these high-quality biospecimens; however, in a busy hospital environment it is hard to define the best time to approach and recruit potential research participants. Routinely, tissue bank staff attend surgical preadmission clinics (PAC) with the aim of consenting patients; however, due to the heavy workflow at PAC, our time and space with patients to discuss tissue banking can be very limited, making the informed consent process challenging. To mediate this ratelimiting step in our biobanking process, the MH Tissue Bank has engaged with PAC clinicians and implemented a process so that tissue bank consent is integrated into the pre-admission process.

Proposed solution: To ensure that clinicians are sufficiently trained to consent patients to the Tissue Bank we provide an introductory talk at each of the education sessions for new rotations of clinicians and provide a written "Guide to Tissue Banking" information pack which contains a copy of our consent form, PICF, and a step by step guide on consenting. We also have an easily accessible tissue bank folder located at the PAC office so that the clinicians can find all the necessary paperwork needed to consent a patient to the Tissue Bank.

Of the 153 breast surgeries that occurred at RMH from January to June 2019, 96 patients agreed to donate tissue for biobanking with approximately $90 \%$ of those consents being obtained by clinicians at PAC. In addition, $32 \%$ of cases with pre-operative consent were targeted for fresh banking, and tissue was obtained from $87 \%$ (27) of the cases.

Conclusion: The process of working collaboratively with clinicians at PAC has largely improved the efficiency in recruiting patients for the Melbourne Health Tissue Bank in a streamlined manner within established hospital practices. This community engagement is helping to ensure that the MH Tissue bank can continue to bank quality biospecimens for the wider research community.

\section{PF-06 Utilizing Social Media and Crowd Activation in Local and National Events to Drive Qatar Biobank's Public Recruitment}

M. H. Al Dosari

Qatar Biobank for Medical Research, Doha, Qatar

Background: Qatar Biobank aims to recruit 60,000 Qatari nationals and long-term residents, 18 or older. More than 28,000 participants have already been recruited through its cohesive recruitment strategy. Qatar Biobank has recently started the followup visits for those who have completed five years since their first registration, and launched MRI to its portfolio of check-ups to generate more insights about the participants' health. Qatar Biobank has been using a multi-pronged participant outreach strategy, powered with proactive public relations activities, to disseminate the underlying messages to the right target audience. The core pillars of the participants outreach strategy includes: participation in industry events, awareness programs, and national events; sustained media outreach; and continued social media activities.

Methods: In addition to monthly social media campaigns, Qatar Biobank participates in monthly media interviews with both broadcast media and print media. Qatar Biobank regularly attends relevant industry events and global awareness campaigns to raise awareness about biobanking. It has been providing opportunity for media and social media influencers to take tours of the facility and personalize their stories. A series of campaigns around the main national events such as Qatar's national day, the national sports day, and the country's landmark national sports day were launched to humanize the Qatar Biobank story, allowing the population to understand the concept better encouraging people to register.
Results and Discussion: Qatar Biobank public participant recruitment strategy and methods successfully achieved to enroll more than $33 \%$ of the targeted population of in less than six years with a waiting list of over 5,000 potential participants. Basing the core messages of the communications activities on the benefits personalized medicine brings to the people of Qatar has been of utmost help to the success of the campaign.

\section{PF-07 Sharing of Biomaterials and Data for Biobank Use in Exchange for Funding in South Africa in International Collaborative Health Research: An Ethico-legal Analysis}

M. Maseme M,2 $^{1,2}$

${ }^{1}$ Biobank, National Health Laboratory Service, Roodepoort, Gauteng, South Africa, ${ }^{2}$ Steve Biko Center for Bioethics, University of the Witwatersrand, Johannesburg, South Africa

When sharing of biomaterials and data is contingent to funding, this in itself could negate the ethical notion of benefit sharing and lean towards commodifying human biomaterials and associated data. None of the current laws and regulations in South Africa address the sharing of biobank samples and data where funding is concerned. Hence, the development of an ethico-legal framework that addresses sharing of biomaterials and data where funding is concerned is necessary. In this research the practice of access and transfer of biomaterials and data from biobanks in international collaborative health research in exchange for funding was critically analysed with the use of principles and theories of ethics and relevant laws and regulations. Pertinent issues explored included exploitation and objectification as a result of commodification, benefit sharing, and ownership of biomaterials. While international guidelines provide a platform that allows for collaborators from different legal systems to work together, legally binding frameworks are useful and hence inadequacies in national regulations must be addressed. It is unethical to share biomaterials and data in exchange for funding because this has the potential for exploitation of the participants due to a lack of respect for their dignity and rights.

\section{PF-08 The Incidence of Participant Withdrawal in the Qatar Biobank Cohort Study}

G. A. Deyab

Qatar Biobank, Doha, Qatar

Background: Obtaining informed consent is an ethical obligation which gives the participant the right to decide whether to participate in a research study. Qatar Biobank (QBB) uses a broad informed consent to explain the research process and provide information to the participant about what to expect and what participating will involve. The informed consent maintains a participant's autonomy and will highlight the research study's risks and benefits. Participant withdrawal is the right of the participant to exit a research study without giving a reason. Qatar Biobank has created a withdrawal procedure to help guide staff and participants who wish to discontinue their participation within the study. QBB has six options available for withdrawal and participants can select more than one. The six options vary from total withdrawal and destruction of data/samples to simply withdrawing with no further action.

Methodology: Two data extractions were requested to identify the number of participants who had completed the withdrawal form and chose to exit the study or who entered the database but did not sign the informed consent form. 
Results: The findings shows that $0.08 \%$ of the participants withdrew from the study using the withdrawal process. The most commonly cited reason was that they did not want to participate in a research project or have their samples stored. The statistics show that $1 \%$ of participants have been registered into the database, but did not sign the informed consent; $63 \%$ of the participants did not continue for reasons related to consent, including not wishing to contribute in the research program or not wanting their samples to be used for research purposes; and $2 \%$ of the total number of the participants were requesting tests not be included in the QBB visit. A further 5\% did not want to wait for the expected length of time for their result and wanted to receive their result feedback within a week, suggesting possibly that they viewed their participation as more of a health check-up than contributing to research.

Conclusion: Giving the participant the right to withdraw from the research study is an ethical requirement in all human subject research; it plays a major role in protecting the participant's autonomy and ensuring the participant has control over participation, data, and samples. The reluctance to participate in research given by some people is a point for consideration in how $\mathrm{QBB}$ and the work of QBB is understood by the general public.

PF-09 Best Practices for Ensuring Regulatory Compliance of Research Tissue Banks in an Academic Medical Centre

J. Yeo, M. Koh, W. Phang

Research Integrity, Compliance and Ethics, Singapore Health Services Pte Ltd, Singapore

Statement of the Problem: In Singapore, all research using human tissue is regulated under the Human Tissue Framework of the Human Biomedical Research Act (HBRA). Any individual or organization involved in removal, collection, storage, supply, import, or export activities of human tissues for future research will be required to comply with the Human Tissue Framework for tissue banking. As standardized, high-quality tissue banks are critical for progress in research, best practices for collection, handling, storage, and shipping of these human tissues are critical. A yearly declaration of compliance by the tissue bank to the Singapore regulatory authority is required for the tissue banking activities conducted. Besides safeguarding the rights and welfare of the donors, the enforcement of compliance ensure accuracy and reproducibility of research based on these tissue banks.

Proposed Solution: In order to assess the compliance level, an assessment tool is being developed for the Academic Medical Centre which housed a few tissue banks. Besides evaluating the quality and integrity of tissue handling (collection, processing, and storage), this tool will assess 1) whether informed consent was taken and the informed consent process, 2) extent of involvement of vulnerable donors, 3) the tissue release governance, 4) database and inventory tracking system of tissue bank, 5) transfer and /export of tissues, 6) handling of privacy and confidentiality of donors, and 7) management of untoward occurrence such as contamination. Each of the tissue banks will be monitored and the deficiency areas will be reviewed.

Conclusions: Based on the preliminary gaps identified, training and education will be developed to address the needs. This allows the Academic Medical Centre to improve the overall regulatory compliance of tissue banking activities.

\section{PF-10 What Did I Just Sign up For? Why Consent is Not} Always Clear-Cut

S. J. Wicks, M. King, J. Lowery, K. C. Barnes, K. R. Crooks
Colorado Center for Personalized Medicine, University of Colorado, Aurora, Colorado, United States

Background: The Colorado Center for Personalized Medicine (CCPM) is enrolling and collecting samples from patients to hasten discoveries in personalized medicine. The CCPM established a biobank laboratory to serve as both a research biorepository and a CLIA-certified genetic testing laboratory. A number of studies to date, as well as feedback from CCPM Biobank subjects, confirm that most research subjects express a preference to receive individual genetic results. Using a selfconsent form with supplemental information provided on our website, CCPM Biobank subjects may choose to have validated clinical genetic test results returned. To understand subjects' motivations for agreeing or declining to receive genetic test results and to identify any gaps in knowledge or misinformation, the CCPM Biobank surveyed a number of subjects.

Methods: One hundred subjects enrolled in the research arm of the CCPM Biobank study were mailed a consent form offering return of genetic test results and then contacted by telephone to complete a survey. The survey collected demographic information and asked subjects to describe: what the consent form requested of them, what results consented subjects would receive, reasons for agreeing or declining the consent, if any parts of the consent were difficult to understand, their decisionmaking process, need for additional information, and alternate methods for delivery of consent. Three CCPM staff members independently assessed the responses to the survey questions for subject understanding.

Results and Conclusions: Thirty-five subjects completed the survey; $23 \%$ of participants were assessed as having fully understood what the consent was asking of them. Subjects assessed as having the lowest level of understanding either did not recall the consent or did not fully understand that individual genetic test results could be returned to them. Interestingly, no subjects said they had difficulty understanding any part of the consent form. Motivations for signing the consent were complex, although many subjects were motivated by curiosity or concern for family health. The results from our survey show that although the consent was designed to be easily understandable, recall of the consent and its meaning was limited in many cases. We recognize that signing a consent is only the first stage in an ongoing relationship with subjects, and we are using the survey responses to help design and improve subject information for return of genetic test results.

\section{PF-11 A Full Understanding of Data Sharing and Protection Regulation in Biorepositories: Observations from a Small LMIC Biorepository in South Africa and Other African Countries}

\author{
C. Swanepoel ${ }^{1,2}$, R. Adams ${ }^{3}$, T. Croxton ${ }^{4,5}$, D. Kamuya ${ }^{6}$,
} M. Munene ${ }^{6}$, D. Anderson ${ }^{7}$, C. Staunton ${ }^{8}$

${ }^{1}$ Division of Haematology, National Health Laboratory Services, Tygerberg, Western Cape, South Africa, ${ }^{2}$ Pathology, Division of Haematology, Faculty of Medicine and Health Sciences, Stellenbosch University, Tygerberg, Western Cape, South Africa, ${ }^{3}$ Institute of Advanced Legal Studies, School of Advanced Study, University of London, London, United Kingdom, ${ }^{4}$ I-HAB, Institute of Human Virology, Abuja, Nigeria, ${ }^{5}$ University of Maryland, Baltimore, Maryland, United States,

${ }^{6}$ Kenya Medical Research Institute, Nairobi, Kenya, ${ }^{7}$ South African National Bioinformatics Institute, University of Western Cape, Bellville, South Africa, ${ }^{8}$ School of Law, Middlesex University, London, United Kingdom 
The Protection of Personal Information, Act 4 of 2013 (POPIA), which will come into force in 2020, will have a considerable impact on how health and genomic data will be processed, and the conditions under which it may be shared, especially for biorepositories. South Africa (SA) lacks a coherent legislative framework and the approach to regulation of data protection and sharing for genomic research and biorepositories in SA is fragmented and requires complex navigation for researchers, and at times lacks consistency. Thus a full understanding of the regulations is necessary to ensure that biorepositories in SA are compliant with their data protection obligations.

Methods: Both current and prospective partners' regulatory frameworks will be taken into account. Therefore NSB current data sharing and protection policies along with POPIA and health- and research-related guidelines will be reviewed as a case study. Partners from various African countries such as Kenya and Nigeria will also be liaised with in order to analyse and review their data sharing and protection legislations to account for differences. We developed/updated a SA/African data sharing and protection summary of dos and don'ts, taking into account all the required national and international legislation.

Preliminary results: Using POPIA as a reference, a coded sheet was generated of key points. Main focus points related to: 1) informed consent and REC roles, 2) MTA and DTAs, 3) the use of secondary data, 4) long-term storage, 5) protection, and 6) in-between countries sharing along with 7) who takes responsibilities in case of data breaches. The different sections covering the various points for the different countries will be summarised accordingly.

Discussion and conclusion: With the coming into force of the GDPR and the soon-to-be implemented POPIA, many research laboratories and biorepositories had to introduce or update legislation to ensure compliance. However, for researchers and biobankers these legislations are a challenge to interpret, taking into account the many differences. Therefore, the summarised guideline for low-and-middle-income African countries that would be generated focusing on the dos and don'ts related to data sharing and protection aims to help researchers and biobankers to become POPIA/GDPR complaint and to create awareness within their own setting.

\section{PF-12 The New Possibility of Regulations on an Example of Polish Code of Conduct for Processing Personal Data for Biobanks Based on Art 40 GDPR}

\section{Krekora-Zajac}

Faculty of Law and Administration, Warsaw University, Warsaw, Poland

General data protection provisions provide a unique opportunity for legal regulations regarding the processing of data for biobanking purposes. This special instrument is a code of conduct. The theme of the presentation will be the proposal of the Polish code of conduct in the field of personal data processing by biobanks as an example of the great possibilities of the self-regulation mechanism. In the code, after long discussions with the biobank community, scientists from medical universities, doctors, bioethics, lawyers, and patient organizations, we have rules not only governing the obligations of the data processor and administrator, but also provisions on the rights of participants, closing biobanks, and re-use of data. At the presentation I would like to describe the code preparation process, its layout, and main principles.

\section{PF-13 Informed Consent in Biobanking: Results of a Systematic Review}

C. Lewis ${ }^{1}$, R. McCarter ${ }^{1}$, R. Fallis ${ }^{2}$, H. Fisher ${ }^{3}$, J. McCarroll ${ }^{4}$, E. McFerran ${ }^{2}$, J. Reid $^{2}$, J. James ${ }^{1}$

${ }^{1}$ Northern Ireland Biobank, Belfast, Northern Ireland, United Kingdom, ${ }^{2}$ Queen's University Belfast, Belfast, United

Kingdom, ${ }^{3}$ Northern Ireland Cancer Research Consumer Forum, Belfast, United Kingdom, ${ }^{4}$ R\&D, HSC Public Health Agency, Belfast, United Kingdom

Background: There is much debate within the biobanking literature on different approaches to consent, particularly whether biobanks should adopt a 'broad' or a 'narrow' model of consent. A number of studies have sought to investigate these approaches but they have had varying conclusions and it is not clear which results are the most reliable and which model should be adopted as best practice. Therefore, there is a timely need to undertake a high-quality, systematic review of the biobank consent literature to identify an evidence based approach to consent in biobanking.

Methods: The Preferred Reporting Items for Systematic Reviews and Meta-Analyses (PRISMA) guidelines were used to conduct the review. Articles were eligible for inclusion if they were published in English and included original empirical data (qualitative or quantitative) where adult participants had been asked about, or had been involved in, the biobank consent process. Electronic searches were conducted of four databases: CINAHL, MEDLINE, EMBASE, and Web of Science. Following removal of duplicates, 369 records were screened and 151 full-text articles were assessed for eligibility and quality. The main findings were incorporated into a qualitative synthesis given the heterogeneity of study designs.

Results: Sixty-two papers were included in the final review. Main themes included Broad Consent, Narrow Consent, ReConsent, and Consent for Residual Clinical Material. Broad consent for prospective sample banking was the overall preferred model of consent; in the small number of papers where narrow consent was favoured this was often associated with concerns regarding data security and privacy. Re-consent was only considered necessary when the proposed use of the sample deviated from the original consent given. There was less consensus regarding an opt-in or optout approach for the use of residual clinical material in research.

Conclusions: The findings of this review support the use of a broad model of consent in biobanking compared to a narrow model of consent. More research is required which specifically relates to consent for the use of residual clinical material.

\section{PF-14 The McCain GU BioBank: Evaluating Approach Metrics and Setting Standards for a Successful Consent Rate}

A. L. Bolanos ${ }^{2,1}$, T. Adeyemi ${ }^{2,1}$, S. Nguyen ${ }^{2,1}$, S. Paul ${ }^{2,1}$, M. Kenk ${ }^{2,1}$, N. Fleshner ${ }^{2,1}$, H. Wagner,

${ }^{1}$ Surgical Oncology, Princess Margaret Cancer Centre University Health Network, Toronto, Ontario, Canada, ${ }^{2}$ McCain GU BioBank, Toronto, Ontario, Canada

Background: The McCain GU BioBank (MGB) is a biorepository storing specimens collected from consenting participants attending out-patient clinics within the genito-urinary (GU) oncology division at University Health Network. Since its inception in 2008, the GU Biobank has supported clinical trials and biomarker development research in Canada and internationally by performing unbiased protocol-based serial collec- 
tions of blood and urine samples. The McCain GU BioBank has maintained an average consent rate of $93 \%$, with $16,000+$ patients consented to the program since 2008 .

Methods: Data were analyzed to evaluate trends in the biobank's consent rate over the last 10 years, focusing on the $7 \%$ of approached individuals who refused to provide consent, examining reasons for refusal, hospital site or clinic where the participant was approached, and number of collections/specimens involved as per protocol. The consent and refusal rates were tracked using an electronic form linked to the biobank's participant database. An analysis was done to determine the percentage of consent, refusal, or undecided decisions at the time of approach. Trends over time were compared with key events like the incorporation of new clinics or collection sites, new collection protocols (e.g., whole genome sequencing), modifications to the consent form, and others, to determine the impact these changes have had on such rates.

Results: The reasons provided for refusing to participate, roughly constant over time, include: lack of interest in participation in research initiatives, concerns with privacy/confidentiality, and unwillingness to donate additional specimens. An increase $(6 \%)$ in refusals due to privacy and/or confidentiality concerns was observed over time. Language of the consent form has been modified and staff were provided training on how to clearly describe academic and non-academic collaborations, present the potential for commercialization of samples, and describe measures used to protect personal information.

Conclusion: The growth in privacy, confidentiality, and commercialization concerns among participants reflects the need to change our perspective and implement strategies as a biorepository to stay current with patient needs. By incorporating new metrics for the rate of participant consents versus refusals and optimizing the informed consent form, the McCain GU Biobank hopes to increase its consent rate further and remain an industry leader in uro-oncological biospecimens.

\section{PF-15 The Implementation of Singapore's Human Biomedical Research Act (HBRA) Human Tissue (HT) Framework in an Academic Institution Setting to Regulate Tissue Banks and Tissue Banking Activities for Research}

\section{J. G. Tan, W. Tan, F. Gan, A. Hor, C. Eng \\ National University Health Systems (NUHS), Singapore}

Singapore's Human Biomedical Research Act (HBRA) defines the roles and responsibilities of individuals and institutions involved in human biomedical research and the handling of human tissue for use in research to protect the safety and welfare of research subjects and tissue donors. The human tissue (HT) framework of HBRA, activated on 1 November 2019, includes provisions to regulate tissue banks and tissue banking activities for research. Here we described the implementation of a single mothership model for HT framework in an academic institution (National University Health System, NUHS) for reporting biobanking activities to the Ministry of Health Singapore. This involves harmonizing policies, procedure, and auditing of satellites biobanks prior to the annual submission.

\section{PF-16 Writing an Easy-to-Understand Biobanking Informed Consent Form in Plain Language}

\section{H. Ellis}

Biobanking Without Borders, LLC, Durham, North Carolina, United States
Problem: In the U.S. the average person reads at below the 8th grade level. Yet all too commonly, consent forms are written at a grade level above high school, in language that is difficult to understand, with lots of legalese to fulfill regulatory requirements. Template language provided by institutional review boards (IRBs) and other resources often miss the mark in terms of readability and grade level. Inserting a paragraph copied from a government website written for scientists does not help 'inform' our participants. Modeling your consent form after a colleague's approved consent form may save time and guarantee IRB approval, but can result in redundant and even conflicting information. Concepts such as GINA and Certificates of Confidentiality are confusing to most, and describing them may not add value to a consent form. I barely understand the difference between genetic, genome, somatic, etc.- so how to explain them to someone else? Furthermore, researchers often tout that they have a 'short consent form' but they achieved brevity by using small font, even smaller margins, and then provide key information in a brochure, much of which should actually be in the consent form.

Proposed Solutions: There are many published guides and tools to help researchers write a comprehensible, reasonablelength consent form. Approaches such as using clear headings, logical organization, one concept per paragraph, short sentences, lots of white space, simple graphics, simple words, and conversational language are easy to implement and have an amazing impact on the clarity, brevity, and readability of consent forms. This presentation will provide easy-to-implement, stepby-step instructions to transform your biobanking consent form into one that your participants will understand, based on published plain language and health literacy principles. Attendees will learn the important concepts and principles, evaluate 'before and after' documents and have hands-on experience in revising real examples of real consent form phrases and concepts.

Conclusion: The informed consent form is only one part of the informed consent process; however, the form may be the only written document that a participant takes home with him or her to refer to later. As researchers we owe it to our participants to provide them with an informed consent form that they can understand, at a time when many of them may be sick, tired, and stressed, yet willing to make the effort to participate in research for the greater good.

\section{PF-17 Returning Individual Genetic Results in a Biobanking Study: Considerations for Participants, Researchers, and Clinicians}

\section{H. Ellis}

Biobanking Without Borders, LLC, Durham, North Carolina, United States

In the field of biobanking, especially in which consent is obtained for unknown future use, providing individual genetic results to participants is a complex and evolving issue. As a participant, most of us would welcome, if not expect, the opportunity to receive relevant test results potentially vital to our health. But as scientists, we know the issues are multifaceted and spawn legal, ethical, and financial dilemmas for researchers, institutions, participants, and their families. One must remember the context under which these results are generated and that the objectives and responsibilities in the research realm are not the same as in the clinical realm. This talk will extend beyond the basic topics of clinical validity, clinical utility, and actionability and delve into issues such as the responsibility of the researcher, timing of consent vs. testing, the level of detail in clinical consent vs. research consent for genetic testing, the challenges of maintaining contact with participants, and objective and 
subjective factors that influence a participant's choice. The talk will also advocate for the inclusion of genetic counselors in the planning and implementation of a biobank, due to the benefits they provide to clinicians, patients, and their families and in understanding the risks and benefits of genetic testing.

\section{PF-18 A Survey of Pregnant Women and New Moms Regarding Their Opinion of Research, Biobanking, and the Consent Process}

S. Vercauteren ${ }^{1}$, A. Ellis ${ }^{1}$, M. Dittrick ${ }^{1}$, P. Muir ${ }^{1}$, P. Lavoie ${ }^{2}$, Q. Aujla ${ }^{1}$

${ }^{1}$ Department of Pathology and Laboratory Medicine, BC Children's Hospital, Vancouver, British Columbia, Canada, ${ }^{2}$ BC Women's Hospital, Vancouver, British Columbia, Canada

Background: Recruiting maternity patients to take part in research can be challenging as it is highly dependent on appropriate timing. The BC Children's Hospital BioBank (BCCHB) recruits patients of $\mathrm{BC}$ Women's Hospital $(\mathrm{BCWH})$ to donate maternal samples such as placenta, cord blood, and maternal blood. Ideally, consent is obtained well before delivery, though this necessitates confirmation of consent closer to delivery to verify continued agreement. In addition, there may be numerous research projects relevant to each patient. We hypothesized that women are interested in participating in research but are not provided the opportunity. We conducted a brief survey to gather feedback regarding the $\mathrm{BCCHB}$ and research consenting processes. Results will inform research recruitment processes of the BCCHB.

Methods: Survey links were distributed through social media channels and to current BCCHB participants. The survey consisted of 24 questions with Likert scale for assessment. Only completed surveys were analyzed.

Results: If 141 completed surveys, 50 women had previously consented to the BCCHB. Preliminary results show that $91 \%$ of women agree on the importance of research at $\mathrm{BCWH}$ but over half have not been asked take part in research. Similarly, $90 \%$ of mothers believe it is important that BCWH supports a biobank of premature births, yet $67 \%$ have not heard of the Preemie BioBank, Canada's first biobank of this type. Further, $87 \%$ of respondents would agree to donating leftover biological samples to a biobank. Of all respondents, $70 \%$ stated they would prefer a single overarching consent either with or without a summary sheet if several studies are conducted at the same time and $80 \%$ of women indicated they would prefer to have BCCHB staff administer the consent form, but $83 \%$ of participants would like the concept of the BioBank to be introduced to them by someone in their circle of care first.

Conclusion: The majority of pregnant women or new moms are supportive of research and are willing to donate leftover samples to research. Increasing awareness may facilitate increased participation and stakeholder engagement in research. Implementing a single consent covering the BCCHB as well as other research studies is an acceptable method which may aid recruitment while decreasing patient burden.

\section{PF-19 Revised Ethical Guidelines In Indian Biobanking: Do We Need To Downregulate the Proposed Frameworks?}

J. Tayal ${ }^{1}$, A. Mehta ${ }^{2}$

${ }^{1}$ Biorepository, Department of Research, Rajiv Gandhi Cancer Institute and Research Centre, India, ${ }^{2}$ Department of Laboratory Sciences and Molecular Diagnostics, $R G C I \& R C$, India
Clinical biobanks are gaining popularity and revolutionizing research in India. The consenting process forms the fundamental of the guidelines directing biomedical research in India. Indian Council for Medical Research (ICMR), Council for Scientific and Industrial Research, and Department of Biotechnology are the major agencies supporting research in India. The ICMR is the national organization and also the apex body for developing ethical frameworks and guidelines and also enforcing them. The ICMR issued the Policy Statement on Ethical Considerations Involved in Research on Human Subjects in 1980. Due to rapid advancement in biomedical sciences new ethical dimensions have emerged and necessitated the update of these guidelines time and again in 2000, 2003, 2013, and in 2017. These guidelines are not only considered as the gold standard by many stakeholders but are also looked upon by many developing countries for guidance.

The National Ethical Guidelines for Biomedical and Health Research Involving Human Participants released in October 2017 introduced many new sections and also revamped the existing sections. A new Section 11 was dedicated to biological materials, biobanks, and datasets. The new guidelines protect the research participants from exploitation, harm, and injustice. However, there is a gross mismatch when it comes to the practical applications of these guidelines in culturally and ethnically diverse countries like ours. When exercising informed consent various factors come into play: at the participant level (poverty, illiteracy, patriarchal societies, and cultural issues) and at the level of the type of biobank (population-based, tumor biobanks, post mortem, gene banks, cell lines bank, etc.).

The need of the hour is to develop a document that not only protects the research participants but also promotes research in the true spirit of altruism. The present guidelines need serious rethinking to answer questions like: Is ICF valid in biobanking setups or would authorization be more appropriate A mandatory pre-banking consent, reconsenting for extended use/reuse, and return of research results are all factors to consider. It is not too great a claim to say that biobanks require new ethical structures and frameworks and not the ones which we have applied generally to other issues in ethics. It is time to regulate the guidelines for Indian Biobanks and devise a single universal framework.

\section{PF-20 Neglected Ethics in Biobanking: Addressing Issues of Stewardship and Utilization}

\section{K. B. Brothers ${ }^{3}$, J. Cadigan ${ }^{2}$, A. J. Goldenberg ${ }^{1}$}

${ }^{1}$ Department of Bioethics, Case Western Reserve University, Cleveland, Ohio, United States, ${ }^{2}$ Department of Social

Medicine, University of North Carolina, Chapel Hill, North Carolina, United States, ${ }^{3}$ Department of Pediatrics, University of Louisville, Louisville, Kentucky, United States

Background: The promotion of precision medicine has led to increased interest in larger collection of biospecimens that are more representative of genetic variation across and within populations. There is now a robust literature identifying and addressing the ethical, legal, and social implications of biobanking. Nevertheless, the recent evolution of biobank networks has revealed a number of unanswered and understudied questions regarding stewardship and utilization of biospecimens and data, including 1) the discrepancy between limited funding and the assumption that the biobank will last "permanently," 2) the lack of planning for what will happen to the specimens and data if the bank terminates, and 3) underutilization of specimens and data. Since that time, the biobanking industry has grown more complex, with increasing numbers of biobanks forming networks. In theory, these networks should facilitate increased stability of operations and utilization. 
Methods: This poster will review the results from a study that investigated to what extent, if any, current U.S. networked biobanks seem to be achieving increased stability and utilization. In 2018-19, we conducted interviews with 37 representatives from networked biobanks. Questions elicited descriptions of the genesis of the network, the challenges and benefits of networking, the utilization process of specimens and data, and the funding sources.

Results: We analyzed transcripts to ascertain how the three concerns highlighted in our prior research on biobanks play out in the context of networked biobanks. Preliminary results reveal that biobank networks have a range of ways of addressing sustainability and utilization with mixed results.

Conclusions: Although networking of biobanks ought to increase the stability and utilization of biobank resources, networks still struggle with these goals. We will use our results to offer recommendations to consider. We will also discuss the implications of our findings as they relate to ensuring public trust in networked biobanking. Finally, we will give ISBER members the opportunity reflect on the creation of models of stewardship that honor donor's desire to see their samples and data used for health research at a time when many biobanks struggle with underutilization by researchers.

\section{Hot Topics}

\section{PG-01 Integration of Cervical Cancer Screening and Prevention into HIV Care at the Family AIDS Care and Education Services (FACES) Clinics in Abuja, Nigeria}

\section{O. Akinbade}

Hematology and Infectious Disease, Jolade Research Initiative, Lagos, Lagos, Nigeria

Background: HIV-infected women are at higher risk of human papillomavirus infection, cervical pre-cancer, and invasive cancer. The risks are increased in limited-resources settings, where cost and infrastructure requirements limit access to traditional cytology-based screening programs. Integration of cervical cancer screening and prevention (CCSP) services into HIV care service may be a feasible and effective way of impacting a high-risk population of women.

Method: In October 2010 CCSP was integrated into routine care at the family AIDS Care and Education Services (FACES) program in Abuja, Nigeria, during regular visits. Non-pregnant women over 25 were invited to undergo cervical cancer screening using visual inspection with acetic acid (VIA) followed by colposcopy and biopsy to confirm positive result. Women who tested negative were re-screened every three years. Treatment of cervical intraepithelial naoplasia 2/3 (CIN 2/3) was done with loop electrosurgical excision procedure (LEEP) on site, with referrals given for more advance diseases.

Results: Between October 2010 and April 2015 we screened over 94,567 women using VIA and diagnosed 1,657 case of CIN 2/3. We performed 1,243 LLEPS with no serious adverse events. We have trained 890 clinical officers and nurses to perform VIA and 80 clinical and medical officers to perform LEEP. Based on the program success, services are now being scaled up to outpatient facilities in multiple districts throughout the Maitama province in partnership with the Nigerian Ministry of Health

Conclusion: This is an example of successful integration of cervical cancer screening and prevention services with an HIV care setting. Building local capacity while providing services to prevent invasive cancer, screening using low-cost, easy-train approaches can result in high coverage of HIV-positive women, with follow-up nested within ongoing HIV care services. With continued mentorship, mid-level HIV care providers can carry out the cervical cancer screening techniques safely and effectively

\section{PG-02 Influence of DNA Methylation on the Antidepressant Effect of Ketamine}

\section{G. Zhang, G. Wang, M. Liu}

The National Clinical Research Center for Mental Disorders \& Beijing Key Laboratory of Mental Disorders, Beijing Anding Hospital, Capital Medical University, Beijing, China

Background: Ketamine is a high-affinity non-competitive NMDA (N-methyl-D-aspartic, N-methyl-D-aspartic) receptor antagonist. In recent years, studies have found that ketamine has rapid onset antidepressant effect. However, the use of ketamine for antidepressant treatment has the risk of causing adverse reactions such as psychiatric symptoms, addiction, and so on. The aim of this study is to find DNA methylation sites related to the efficacy of ketamine and screening-related genes. It will be the foundation of the accurate use of ketamine for depression.

Methods: This study included a randomized, double-blind, placebo-controlled study of brain tumors with depression. The subjects received a small dose of ketamine intravenously $(0.5 \mathrm{mg} / \mathrm{kg}, 40$ minutes $)$ during the operation. The MontgomeryÅsberg Depression Rating Scale (MADRS) was used to assess depression. According to the improvement of depression on the third day after operation, patients were divided into an effective group (MADRS score reduction rate is greater than or equal to $50 \%$ ) and an ineffective group (MADRS score reduction rate is less than 50\%). Illumina Infinium Methylation EPIC Bead Chip (850K DNA methylation chip) was used to carry out genomewide methylation of peripheral blood in two groups. The methylation sites with significant difference between the two groups $(p<0.05)$ were compared. According to the difference of methylation beta and the difference score, bioinformatics analysis was carried out using GO analysis and Pathway in Kyoto Encyclopedia of Genes and Genomes to select chloramine. Ketone therapy is associated with different DNA methylation levels.

Results: A total of 34 subjects using ketamine were included in this study, of who 20 were in the ineffective group and 14 were in the effective group. A total of 34,140 differentially methylated sites were identified by $850 \mathrm{~K}$ DNA methylation chip screening. These differentially methylated sites were located on 13,209 genes. Through bioinformatics screening, we have identified 39 genes related to multiple pathways as the basis for subsequent research.

Conclusion: It was found that many metabolic and signaling pathways were involved in the factors affecting the efficacy of ketamine. Through bioinformatics analysis and literature review, 39 anti-ketamine drugs were screened out. Genes related to the efficacy of depression lay the foundation for future research.

\section{PG-03 Biobanks in a Primary Care Setting: What Is Missing?}
A. Salman ${ }^{2,1}$, A. Lazaris ${ }^{1}$, P. Metrakos ${ }^{1}$, G. Bartlett ${ }^{2}$
${ }^{1}$ McGill University Health Centre, Montréal, Québec, Canada,
${ }^{2}$ Family Medicine, McGill University, Montréal,
Québec, Canada 
Population-based biobanks are designed to link biomarkers with medical history and lifestyle information. Biobanks in primary care (PC) will support a better understanding from its users and service providers as well as the complexities associated with chronic diseases including multiple co-morbidities, spectrum of disease from symptom to mortality, and the human life cycle. Today, there are no PC-focused biobanks that we know of. In this study, we evaluate the gap PC-based biobanks might fill by completing a narrative review to provide a comprehensive, critical, and objective analysis of the current knowledge around population-based biobanks. The scope of the review is limited to published and unpublished works, and works in English between 1998 and 2017. The search returned 2,067 articles cited in PMC. We excluded 1,437 articles. A total of 630 articles were included in our study where we identified 136 biobanks in which 41 population-based biobanks were identified. The biobanks identified with the most articles published in our series were the UK Biobank, Guangzhou Biobank, and the China Kadoorie Biobank. Our review focuses on characteristics observed in all three biobanks. The most comprehensive type of population-based biobank is designed to link biomarkers with medical history and lifestyle information as well as rare diseases that result in single gene mutations but without any collection of prospective data at the primary care level. All three biobanks have a follow-up that is based on electronic medical records and national mortality registries. The collection of data and biospecimens are performed during specific routine visits with the participants. Therefore, we are missing a large amount of clinical, psycho-social, and physician interactions data in primary care. These are some of the challenges observed in the current structure; population-based biobanks do not solve the challenges in research we are currently facing such as data and sample collection in a primary care setting. In the review, we also notice the misuse of biobanking terms can misrepresent population-based biobanks and mislead researchers who seek population-based data or biospecimen from biobanks and not registries or prospective cohort studies. It is important that the biobanking scientific community scrutinizes biobanks on the type of labels they associate their structures and ensure the importance of this distinction as we move rapidly towards precision care medicine at a PC level.

\section{PG-04 Biobank Facilitate the Development of Precision Medicine}

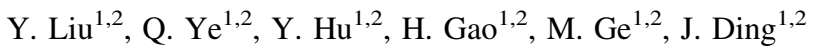

${ }^{1}$ Biobank, Department of Pathology, Nanjing Drum Tower Hospital, The Affiliated Hospital of Nanjing University Medical School, Nanjing, Jiangsu, China, ${ }^{2}$ Obstetrics and Gynecology Disease Biobank, Jiangsu Biobank of Clinical, Nanjing, Jiangsu, China

Statement of the Problem: In recent years, in the context of healthy China, precision medicine and the construction of biobank have ushered in a golden period of development in China. Precision medicine and biobank share the same development trend and characteristics: 1) The development process needs standardization and standardization, 2) it is highly dependent on the construction of informatization, 3 ) it relies on multi-center alliance and community, 4) support of multidisciplinary integration is needed. The implementation of precision medicine plan has brought about the demand for a large number of biological sample resources. In the process of precision medicine development, higher requirements are constantly put forward for the development of biobank, which continuously promotes the development stage of biobank. How can biobank facilitate the development of precision medicine?

Proposed Solution: To provide better and comprehensive support for the development of precision medicine, development of biobank can be divided into the following three stages: 1) The first stage: the platform for integrating biological samples and information resources to complete and realize the "input" function of sample resources and biological information. Through the collection and integration of specialized clinical samples and information, it supports the great demand of translational medicine research on biological samples and information resources. 2) The second stage: the precision medicine research support service platform which focuses on clinical major diseases; establishes screening technology systems such as biochip data analysis, proteome data analysis, and high-throughput bioinformatics of human diseases; and one-stop access to required information and data. 3) The third stage: platform for precision medicine achievement transformation cooperation. It realizes the storage, transmission, and sharing of multiple types of biological sample data; the sharing mechanism and cooperation channel of operable biological sample resources; and information resources.

Conclusions: When the biobank continues to develop to a more mature stage, it also provides better quality and comprehensive support for the development of precision medicine. Eventually realizes the economic value of biological sample resources through the basis of big data of biological information and external scientific research services.

\section{PG-06 Maintaining Sample Integrity during Surface Decontamination of Cryopreserved Materials}

\section{R. Jochim, K. M. Peterman}

Biosafety and Biosecurity Programs, Merrick \& Company, Greenwood Village, Colorado, United States

Statement of the Problem: Cryopreservation is a requirement for many biorepository samples including cells, tissues, and pathogens. Contamination of the outer surfaces of cryopreserved sample containers can have a number of downstream consequences: compromised subsequent culturing, cross-contamination within storage devices, and transfer of contaminated materials. Most currently accepted methods of sanitation involve temperatures and minimum contact times that will compromise cryopreserved materials. For most cryopreserved biological materials, thawing and refreezing of the samples can damage protein structures, nucleic acids, and cell membranes. The freezing process results in salt concentration that can lead to osmotic imbalance and protein precipitation. Temperatureassociated $\mathrm{pH}$ shifts and $\mathrm{pH}$ changes associated with buffer concentration can also damage samples. Cryoprotectants added to samples can alleviate some of these stresses that freezing imparts; however, the repeated freezing and thawing due to surface decontamination processes is detrimental to live sample viability and protein and nucleic acid stability.

Proposed Solution: Sample decontamination methods were investigated and assessed for effectiveness of sanitation and sample preservation. Vapor deposition using vaporized hydrogen peroxide or chlorine dioxide is not a viable decontamination process due to the low temperatures condensing the decontaminant before it is able to coat and sanitize sample container surfaces. Liquid decontaminants considered included sodium hypochlorite; acetic acid; potassium peroxymonosulfate $/ \mathrm{NaCl}$; sodium carbonate; sodium hydroxide; citric acid; and hydrogen peroxide with the addition of anti-freezing agents methyl alcohol, propylene glycol, and calcium chloride. The sample temperature 
change over time at different decontaminant temperatures was calculated. Another method investigated involves the washing of ultra-low temperature samples with sterile liquid nitrogen to remove surface contaminants.

\section{PG-07 Biobanking Trends in Tissue Collection and Release: What's Really Going On?}

A. L. Coulas ${ }^{1}$, M. Fox ${ }^{2}$, M. H. Schild ${ }^{1}$

${ }^{1}$ Department of Pathology, Duke University, Durham, North Carolina, United States, ${ }^{2}$ Department of Information Technology, Duke University, Durham, North Carolina, United States

Background: Historically, a preferred method for processing of human tissue samples has been embedding in optimal cutting temperature (OCT) medium and freezing. OCT-embedded tissue can be used for direct top-slide creation as well as immunofluorescence, immunohistochemistry, and DNA/RNA extraction. The Duke University Health System (DUHS) BioRepository and Precision Pathology Center (BRPC) has been procuring, storing, and releasing human tissue since 2012. In response to increased demand, the DUHS BRPC implemented methods of processing, storage, and release in which fresh tissue is snap frozen in liquid nitrogen or viably stabilized and released in investigator-specific media or cryopreserved. This is important because viable cells can be utilized for flow cytometry, single-cell RNA sequencing, avatar development, and cell culture. Histologic quality control is performed through mirror-face embedding and slide creation. During this time, we simultaneously decreased procurement of OCT-embedded samples in response to a consistently low demand. In this study, we sought to quantify client-driven shifts in human tissue processing and distribution.

Methods: Surgical specimens were collected and processed per BRPC standard operating procedures. Collection requests and releases of OCT-embedded samples versus snap-frozen and viable/cryopreserved samples were retrospectively reviewed from 2014 through 2019. The data were analyzed in two-year increments (2014-15, 2016-17, and 2018-19) to evaluate trends in the collection and release of fresh tissue over this time.

Results: Demand for OCT-embedded specimens was consistently the lowest among all sample types with only 300 samples released in 2018-2019. Correspondingly, OCT collections decreased from 8,281 to 2,086 during the study period. Collections of snap-frozen and viable/cryopreserved samples increased from 196 to 3,716. Release of snap-frozen and viable/cryopreserved tissue samples increased from 29 to 2,325 .

Conclusions: One measure of a successful biorepository is sample utilization in research. Shifting toward procurement of snap-frozen and viable/cryopreserved samples has resulted in increased utilization of specimens from the DUHS BRPC. Now that new technologies allow for the analysis of fragmented RNA and digital-spatial profiling from more plentiful archival paraffin specimens, biorepositories should be intentional about whether they continue to collect OCT-embedded frozen tissue samples.

\section{PG-08 Sell Your Biobank! Outlining a Strategy to Increase Biobank Usage}

E. van Enckevort, M. Swertz

University Groningen, University Medical Center Groningen, Haarlem, Netherlands

Statement of Problem: Biobank collect samples and data for research, but utilisation rates for samples are generally low.
Utilisation rates of around $10 \%$ to $15 \%$ are regularly quoted. While preserving samples long term for future research certainly has its value, better utilisation of already collected samples and data can significantly speed up new research by reducing the time to acquire the necessary samples. However, researchers often do not have insight into the available material in the biobanks or find it too burdensome to request material from the biobanks. Therefore, researchers often opt to collect the necessary samples and data themselves instead of using the already available resources. This creates a significant loss in time and cost in money to set up a new research project.

Proposed Solution: Biobanks need to develop a strategy to make their biobank to the research community. This strategy needs to center around providing researchers the information they need to first find the biobank and to second find the right samples/data for their research and finally to make it easy to request access to the samples and data. There are different not-for-profit solutions already available such as the ISBER Repository Locator (IRL https://irlocator.isber.org/) and the BBMRI-ERIC Directory (https://directory.bbmri-eric.eu/) for searching and BBMRI-NL Podium (https://podium.bbmri.nl) and the BBMRI-ERIC Negotiator (https://negotiator.bbmri-eric.eu/) for requesting samples. Next to this several commercial companies offer services to bring researchers and biboanks together. Registering and maintaining a presence in these systems is an easy way to increase the visibility of the biobank and access to the samples and data.

Conclusion: Biobanks that provide researchers insight in the available samples and data and provide a easy procedure to request access to their material see a higher utilisation rate of the material. As an example, the pathology laboratories in the Netherlands, united in the PALGA biobank, saw an significant increase in the number of succesful requests once they provided the researchers with online tools to find and request material from the Dutch pathology laboratories.

\section{PG-09 Using the Duke Human Heart Repository to Develop Cardiovascular Gene Therapies}

M. Watson, D. E. Bowles

Duke University, Durham, North Carolina, United States

Background: Gene therapy is a methodology to introduce genetic information into cells to compensate for abnormal genes or to make a beneficial protein. While there are two Food and Drug Administration-approved gene therapy products for rare genetic diseases, there has been limited success in cardiovascular gene therapy. Successful gene therapy relies upon four integrated components: 1) molecular targets therapeutic for the disease of interest, 2) a well-defined disease with models for testing, 3) a vector to deliver the nucleic acid), and 4) a delivery approach. Biorepositories such as the Duke Human Heart Repository (DHHR) that contain highly annotated preserved biospecimens and also have regular access to viable human myocardial tissue are uniquely equipped to provide both molecular targets and disease models for testing to expedite translation of cardiovascular gene therapies.

Methods: Molecular evaluation of tissue derivatives (DNA, RNA, protein lysates) contained in the DHHR, combined with bioinformatics evaluations, have identified several molecular targets that may be manipulated in a variety of cardiovascular diseases by gene therapy approaches. AAV or Ad-based viral vectors have been developed around several of these targets. As many of these targets are novel discoveries, and no animal models are available, we are exploiting the DHHR to 1) obtain viable human tissues of disease etiologies of interest, 2) transduce tissue slices with appropriate viral vectors and maintain them in culture, and 3) 
evaluate target protein levels and cardiac function in these transduced tissues at various time points post transduction.

Results: Functional assessment of viable (diseased) human myocardial tissue was evaluated using a Radnoti bath system. Tissues were stimulated to contract using a square wave pulse at $10 \%$ above threshold. Tissues were able to contract, establish a steady baseline, and develop tension. $\beta$ adrenergic stimulation increased developed tension and lowered basal tension (increased relaxation) as expected. Force frequency relationship of the tissues are in line with reported values with developed tension decreasing and basal tension increasing with higher beat frequency. Evaluations of cultured cardiac tissue strips with viral-based gene therapies is ongoing.

Conclusions: There is great value to test therapies in the context of human disease and viable human tissue slices may be used a platform in the evaluation of gene and other personalized medical therapies.

\section{PG-10 Brain Organoids: A Promising Resource of Living Biobank for Neuroscience Research}

\section{S. Li, M. Wang, J. Zhou \\ Shanghai Children's Hospital, Shanghai Jiao Tong University, Shanghai, China}

Biobanking plays an important role between clinical practice and translational research. In addition to the traditional biomolecular-based biobank, there is a growing interest in establishing living biobanks, including organoid biobanks that can collect and store viable and functional tissue and proliferative cell types for long periods of time. An organoid is a threedimensional cell complex derived by self-organization of small tissue blocks or stem cells which can recapitulate the phenotypic and genetic characteristics of targeted human organs. Publications on brain organoids have increased recently, and several types of brain organoids have been reported to model normal and abnormal neural development, as well as different neurodegenerative diseases, neuropsychiatric disorders, and other neural conditions. Based on the current experiments, more exploration on brain organoids is needed, through technical advancements to improve the reproducibility and scalability, as well as to decrease the diversity. Moreover, given the nature characteristics, more ethical attentions are needed considering the extent of maturation and complexity of brain organoids. Living biobanks collecting categories of brain organoids possessing different genetic backgrounds and with spatial and temporal characteristics will eventually contribute to the understanding of neural conditions and ultimately facilitating innovative treatment development.

\section{PG-11 Conditionally Reprogrammed Cell Models Derived From Patients with Pancreatic Cancer for Basic and Translational Research}

\section{S. Agarwal}

Department of Pathology, Georgetown University, Washington, District of Columbia, United States

Historically, it has been difficult to propagate cells in vitro and in vivo that are derived directly from human tumors or healthy tissue. However, in vitro preclinical models are essential tools for both basic and translational research, including drug discovery and drug target identification. Conditional reprogramming $(\mathrm{CR})$ is an in vitro cell technology for generating patient-derived cell line model systems. The method involves co-culture of irradiated mouse fibroblast feeder cells with human epithelial cells in the presence of a Rho kinase inhibitor (Y-27632) using a defined medium. This technology is costeffective, rapid, robust, and reliable. CR-derived cells can be biobanked and used for various basic and translational applications, including regenerative medicine, drug sensitivity testing, molecular and genomic profiling, pathway analysis, and xenograft studies. We have used CR cell technology to establish eight patient-derived cell line models. These cultures are very heterogeneous with respect to chromosome numbers (aneuploidy) and chromosomal translocation spectrum as assessed by spectral karyotyping and multiplex fluorescence in situ hybridization (miFISH). Each of these cell lines carries a different K-Ras mutation for codon 12 and exhibits differential sensitivity to Gemcitabine, SN-38, and Seliciclib. We also used Zebrafish tumor metastasis (ZTM) model to evaluate the sensitivity of Gemcitabine and SN-38 to inhibit migration of tumor cells into the tail region of zebrafish. ZTM is a next-generation in vivo model system to study metastasis that utilizes 48-hour postfertilized embryos. The method is rapid, and allows the real-time investigation of metastasis (migration, intravasation, and extravasation) and can be used as a cost-effective drug-screening platform that works in a very short period of time (3-7 days postinjection). In conclusion, conditional reprogramming, coupled with an in vivo zebrafish model, is an individualized approach to evaluate the effectiveness of chemotherapeutic compounds that can be applied to a wide variety of cancers.

\section{PG-12 Biospecimen Use of Hospital-Based Biobank Over a Decade in China from Renji Report}

\section{Kang}

Renji Hospital, Shanghai Jiaotong University School of Medicine, Shanghai, China

Background: The value of biobank is its supporting role for scientific research, publication, and the transformation which involve many comprehensive factors. Usage analysis will provide valuable instruction to the biobank sustainability. We make a retrospective summary and cost-effective analysis according to the collection and usage of biospecimens of Renji Hospital Biobank in the past 10 years. These data may provide some suggestions to the sustainability for academic hospital-based biobanking.

Methods: We summarized the dynamic statistics data of sample collection, access, and usage as well as research findings from 2008-2018. The performance dynamics were analyzed by correlation analyses according to Spearman.

Results: Renji Hospital Biobank is a centralized interdisciplinary Bank of Biomaterials and Data, which provides central service of human biological material for project-driving academic research. The Biobank comprises a systematic, simultaneous, and sequential collection of body fluids and tissue from patients and study participants of 22 departments in the hospital. The sample includes tissue and fluid samples. The amount of annually collected aliquots rose significantly from 2,000 aliquots per year during 2008 to 2013, 5,132 aliquots (2013) to 174,149 (2018) $(\mathrm{p}=0.015)$. Yearly number of requested aliquots from 561 (2013) to 9,613 (2018). The number of published research articles per year to which the Biobank contributed increased 10 times in a decade, resulting in near 100 identified publications $(\mathrm{p}=0.003)$. The staff training, automatic system, continuous quality improvement, researcher willing and government policies contributed to the quick development. Virtual costs in each requested samples were estimated meanly near $\$ 15$. 
Conclusion: Renji biobank represents a growing resource in China, mainly consisting of project-driven collections of biomaterials, which has already contributed to considerable scientific output. Lacking of accurate citable references was still a challenge to the biobank.

\section{PG-13 Biobanks - Industry Collaboration: How the BBMRI-ERIC Stakeholder Forum is Helping Companies and Biobanks Changing Culture}

\section{F. Florindi, E. Steinfelder}

\section{BBMRI-ERIC, Graz, Austria}

We know the future of healthcare is personalised medicine. The role of biobanks in the development of new personalised medicine solutions cannot be understated: there will be no personalised medicine without biobanking.

However, a divide between researchers and biobanks exists:

- Publicly-funded independent biobanks assume it is selfexplanatory that a researcher would take advantage of their offerings and partner with a biobank when he/she is looking for a reliable source of high-quality samples and associated data to drive their research for the benefit of human health.

- Researchers (from both academia and industry) believe that there are many alternatives to established biobanks. Often researchers are not aware of the existence of biobanks, or the samples stored in the biobanks are not easily findable and accessible, or the researchers simply have a strong wish to have their own collection of specific material.

The result is that only a very small fraction of the valuable samples hold by biobanks are actually used for research. This divide is not new in the world of biobanking. However, the authors will argue that such divide has never been more dangerous than now. New technologies are quickly becoming mainstream, and blurring the borders between research and healthcare: eHealth \& mHealth, digital pathology, and widespread NGS. All these new healthcare solutions rely on sharing of high-quality samples and data that only biobanks can satisfy to the fullest.

In this scenario, the divide magnifies its unwanted consequences and, in the long term, can have a devastating impact on the future of personalised medicine and biobanking. That is why BBMRI-ERIC and 15 industry partners (coordinated by EFPIAEBE) opened a conversation on how to kindle a renewed industry-biobanks cooperation. Under the umbrella of the BBMRI-ERIC Stakeholder Forum, we agreed on the key mission/vision of a position paper that shall define a new contract for industry/biobanks collaboration. The paper does not define a strict framework for collaboration. Instead it aims at providing practical guidance to biobanks and industry on how they can work better together. The document is underpinned only by one higher value: it is unethical for biobanks and industry not to collaborate, and therefore share samples/data, when patients and citizens donate their samples/data with the expectations, often explicit, that they would be used to advance research, while it often is not the case.

\section{PG-14 Biobanking of Whole Blood and Nucleic Acid Samples at Ambient - A Green Solution for Biobanking}

S. Nasarabadi

GenTegra LLC, Pleasanton, California, United States
Sample biobanking becomes necessary when the specimens of interest are precious and especially when used to support clinical trials, epidemiology, and biomarker discovery. The Brain Initiative and the Precision Medicine initiatives recently started are intended to collect a million cohort samples of that kind. Tissue biopsy samples can be and have been banked very effectively for decades as formalin-fixed, paraffin-embedded samples. The other most commonly banked tissue sample for genomics is EDTA blood as it is pristine, minimally invasive, and a readily available donor sample. The common method for storage of such blood is by freezing at $-80^{\circ} \mathrm{C}$. The logistics of storage and retrieval of these frozen samples, as well as the need for power backup in case of an unforeseeable catastrophe, makes ambient (room temperature) storage an attractive option. This option is more relevant when there is a need to store millions of donor samples collected under a national initiative. Storage at ambient temperature and humidity of such donor samples as dried blood spots (DBS) is a paradigm shift from the norm but one which is independent of a reliance on the power grid, has a 100fold lower carbon footprint, and is 100 times more compact and readily suited to automated recovery. Other than biobanking, DBS samples can be an effective and convenient means of collection of samples from participants in clinical trials. The trial candidate would be more compliant to sample collection in the comfort of his or her own home. Blood stabilized on the DBS can then be mailed by local postal services at their convenience. Although storage of whole blood as DBS is an old technology, the adoption of DBS has been hindered by low recoveries and low quality of the extracted nucleic acid. We present here a completely new paperbased technology to overcoming these drawbacks, deployed as an improved DBS collection card and matched nucleic acid extraction method that yields DNA with quality and quantity sufficient to support advanced methods such as next-generation sequencing.

\section{Human Specimen Biorepositories}

\section{PH-01 Quality Assessment and Impact Factors of Tissue and Biofluid Samples in Vietnam CREDCA network}

C. V. Nguyen ${ }^{1}$, L. Nong ${ }^{2}$, T. $\operatorname{Tran}^{2}$, H. M. Nguyen ${ }^{7}$, H. H. Duong ${ }^{3}$, L. S. Nguyen ${ }^{4}$, G. T. Nguyen ${ }^{6}$, L. S. Nguyen ${ }^{5}$, H. A. Nguyen ${ }^{1}$, R. Le ${ }^{1}$

${ }^{1}$ Department of Pathology, Vietnam National Cancer Hospital; Center for Research and Early Detection of Cancer, Hanoi, Vietnam, ${ }^{2}$ Department of Pathology, Vietnam National Cancer Hospital, Hanoi, Vietnam, ${ }^{3}$ Department of Pathology, Hanoi Oncology Hospital, Hanoi, Vietnam, ${ }^{4}$ Department of Pathology, Viet Duc Hospital, Hanoi, Vietnam, ${ }^{5}$ Department of Pathology, Pham Ngoc Thanh Hospital, Ho Chi Minh, Vietnam, ${ }^{6}$ Department of Pathology, Viet Tiep Hospital, Hai Phong, Vietnam, ${ }^{7}$ Department of Pathology, 103 Military Hospital, Hanoi, Vietnam

Background: Maintaining the stability and integrity of DNA and RNA is a major problem in tissue banking. The impact of ex-vivo ischemic time and fixative time on DNA, RNA, and protein expression involved in hypoxia, stress, apotosis, and autophagy remains elusive.

Objectives: The present study aimed to systematically evaluate the impact of ischemic/ or fixative time and storage time on tumor quality based on DNA and RNA levels.

Objects and Methods: A total of collected tumor samples from all sites of CREDCA network in Vietnam range from 2009 to 2018 (up to nine national hospitals). Samples were included in cancer tissue (FFPE and fresh) and peripheral blood (plasma 
and serum). The crude samples were exported to Asterand, a U.S. company for assessment of quality.

Results and Conclusion: There were many types of different carcinomas which were collected from the CREDCA network. A quality check was done. The pass rate ranged from 33 to $100 \%$ depending on the different sites. Some factors, affecting the sample quality, were detected.

\section{PH-02 Somatic Mutation Signatures in Primary Liver Tumors of Workers Exposed to Ionizing Radiation}

D. Goerlitz ${ }^{1}$, J. Blancato ${ }^{1}$, E. Kirillova ${ }^{2}$, C. Loffredo ${ }^{1}$

${ }^{1}$ Georgetown University, Washington, District of Columbia, United States, ${ }^{2}$ Russian Radiobiological Human Tissue Repository (RHTR), Ozyersk, Russian Federation

Background: The Russian Radiobiological Human Tissue Repository (RHTR) at the Southern Urals Biophysics Institute in Ozyorsk, Russia, houses a collection of stored tissues representing the world's largest case series of workers with liver cancer following plutonium exposure. The Mayak worker cohort consists of 25,757 workers, $25 \%$ of whom were female, employed at the Mayak Production Association Facility established by the Soviet Union in 1948 to produce nuclear material for the Russian nuclear weapons program. During operations (1948-1982), workers at the facility were exposed to high levels of ionizing radiation (IR) from inhaled plutonium. Epidemiological studies of the Mayak worker cohort provide direct evidence that occupational exposure to IR increases liver cancer risk. Despite this evidence, little is known about the contribution of IR exposure to the mutational spectrum of liver tumor genomes. Liver tumors from this cohort provide a unique resource of biospecimens to study the influence of IRinduced DNA mutations in primary liver cancer.

Methods: We used whole exome sequencing (WES) to characterize somatic DNA mutations in a sample of three histologically distinct primary liver tumors (angiosarcoma of the liver [ASL; $n=2$ ], cholangiocarcinoma [CCA; $n=2]$, and hepatocellular carcinoma [HCC; $n=3]$ ) from Mayak workers to identify signature mutational events.

Results: WES revealed these tumors harbor an excess of deletions similar to that previously reported in radiationassociated tumors from other studies. These tumors were also enriched for clustered mutations, a signature of radiation exposure. Multiple tumors displayed similarities in abrogated gene pathways including actin cytoskeletal signaling and DNA doublestrand break repair. WES identified novel candidate driver genes in ASL involved in angiogenesis. We confirmed known driver genes of CCA, and identified candidate driver genes involved in chromatin remodeling. In HCC tumors we validated known driver genes, and identified novel putative driver genes involved in Wnt/ $\beta$-catenin signaling and chromatin remodeling.

Conclusions: This study identifies several novel candidate driver mutations that are likely caused by IR exposure, and provides the first data on the mutational landscape of liver cancer after IR exposure. This study also demonstrates the great potential of the biospecimens housed in the RHTR to be used for future experiments using modern "-omics" technologies.

PH-03 Digital Image-Based Data Sharing With or Without Procured Research Tissues, 2015 - 2018

R. Mandt, A. Parwani, D. G. Nohle, L. W. Ayers

Department of Pathology, Ohio State University, Columbus, Ohio, United States
Background: The Cooperative Human Tissue Network is a NCI-sponsored prospective human tissue procurement program that provides quality human tissue and clinical data to approved investigators. Data collection from research tissues is completed prior to shipment to investigators or once available after shipment of fresh tissues. Collected data is maintained in a custom Research Tissue Procurement - Information System (RTP-IS) for future use.

Methods: Tissue Procurement Services procures a quality control (QC) tissue sample reflective of the consented surgical remnant tissue procured for the investigator. Samples are formalin-fixed, paraffin-embedded; cut; and H\&E stained. Stained QC tissues are scanned (ScanScope XT, Leica) and Whole Slide Image (WSI) files are placed on a secure server. The pathologist views the WSI, associated pathology report, and 16 data entry choices from dropdown menus, etc. Percent of target tissue is entered along with \% necrosis and pathologist review result (Pass, etc.). The pathologist approves the final histopathological diagnosis based on standard pathology vocabulary and other specified data. The QC tissue digital WSI, pathology report, and sample descriptive data associate with no PHI or donor identifiers and are stored in a data repository available for additional distribution with or without QC paraffin blocks. A de-identifier can be used to retrieve the case record for expanded clinical data if approved.

Results: Of the 6,877 research tissue samples examined for tissue quality and data entry using glass slides or WSI, $90.6 \%$ passed and $4.0 \%$ failed due to wrong/insufficient requested target tissue in the sample. A total of $5.5 \%$ of digital sample records were pass adjusted on review based on specific histopathologic findings vs initial preliminary diagnosis. The WSI throughput is efficient and significantly contributed 8,649 images of H\&E slides from 5,804 cases since 2012 to the data repository for future use.

Conclusions: Retrieval of WSI through Informatics management provided by RTP-IS records from a data repository is convenient, timely, and avoids the need to transport glass slides from storage for case review. The stored images, paraffin blocks, and clinical data are retrieved for specific downstream investigator requests or digital image research. Data sharing is important to avoid duplicate efforts by researchers and provides the opportunity to immediately review features of the research tissues prior to initiation of laboratory testing.

\section{PH-04 The Russian Radiobiological Human Tissue Repository of the Southern Urals Biophysics Institute}

C. Loffredo $^{2}$, T. Azizova ${ }^{3}$, M. Zakharova ${ }^{3}$, D. Goerlitz ${ }^{1}$, A. Alaoui ${ }^{1}$, E. Kirillova $^{3}$

${ }^{1}$ Department of Oncology, Georgetown University Medical Center, Washington, District of Columbia, United States, ${ }^{2}$ Department of Oncology, Georgetown University Medical Center, Washington, District of Columbia, United States, ${ }^{3}$ Southern Urals Biophysics Institute, Ozyorsk, Russian Federation

The Russian Radiobiological Human Tissue Repository (RHTR) at the Southern Urals Biophysics Institute in Ozyorsk, Russia, has an overall mission focused on biobanking human biological specimens to support research on the long-term health consequences of chronic low-dose-rate ionizing radiation exposures. Two major groups of subjects have been donating such biospecimens, starting in 1951 and continuing to the present time: exposed workers at the Mayak nuclear production facilities, and city residents of Ozersk (controls) who were never occupationally exposed to ionizing radiation. 
RHTR personnel collect tissues, blood, and other types of biomaterial with signed informed consent of participants or next of kin, and all of the specimens are annotated with demographic, occupational, dosimetry, and medical history information. Protocols for specimen collection, processing, storage, and quality control at RHTR are continually updated and brought into compliance with international best practices guidelines. To date, surgical and/or biopsy tissues have been collected and stored from nearly 1,000 individuals, and autopsy tissues and whole organs are available from nearly 1,100 individuals. RHTR also collects and stores blood samples and processes them to yield leukocytes, lymphocytes, immortalized B-cells, erythrocytes, plasma, serum, and blood DNA from workers (6,000 samples) and controls (500 samples). Familial blood DNA is available from parent-offspring triads/diads where at least one of the parents was an exposed worker $(1,500$ families). Additional collections in the RHTR include oral epithelial cells, saliva, induced sputum, and bone marrow from selected individuals (1,500 samples). The biological materials and their annotated data are available to be shared with interested scientists worldwide, via the RHTR web site, which enables interested users to browse a real-time, continuously updated catalog of specimens and data elements. An online application is available to request specimens and to inquire about additional details. Examples will be described during the conference presentation to illustrate the role of RHTR in facilitating state-of-the-art radiobiological research, using this unique biospecimen resource.

\section{PH-05 Use of Samples from NLISSI Biobank for Validation of Genome-Wide Association Studies on Hepatocellular Carcinoma}

S. Ezzat ${ }^{1}$, I. Maged ${ }^{1}$, A. Mosbeh ${ }^{1}$, H. Gad ${ }^{2}$, A. Roshdy ${ }^{1}$, I. Waked ${ }^{1}$, M. Abdel Rahman ${ }^{3}$

${ }^{1}$ National Liver Institute, Shibin, Egypt, ${ }^{2}$ Kasr El Aini, Cairo, Egypt, ${ }^{3}$ Ohio State University, Columbus, Ohio, United States

Background: Hepatocellular carcinoma (HCC) is one of the most common causes of cancer mortality globally. Hepatocarcinogenesis is a complex multifactorial process involving interaction between host and environmental risk factors. Recent genomewide association studies have identified two loci (rs2596542 in MICA and rs9275572 in HLA) in the Japanese population as being associated with the risk of $\mathrm{HCC}$ among chronic hepatitis $\mathrm{C}$ virus patients (HCV-related HCC). There are no sufficient data regarding that association among Egyptians. In the present study, we aimed to determine whether these SNPs are predictive for HCVrelated HCC development in the Egyptian population as well.

Methods: Blood samples were obtained from the National Liver Institute sustainable Sciences Institute Collaborative Research Center (NLISSICRC) Biobank. The NLISSICRC was established in 2012 by a long-term memorandum of understanding between the Sustainable Sciences Institute, San Francisco, United States, and the National Liver Institute, Menoufia, Egypt. SNPs rs2596542 and rs9275572 were genotyped using real-time PCR TaqMan ${ }^{\circledR}$ SNP Genotyping Assays for $491 \mathrm{HCC}$ patients, 485 chronic hepatitis $\mathrm{C}$ patients, and 446 healthy controls. Odds ratio (OR) and $95 \%$ confidence interval (CI) were calculated by logistic regression.

Results: rs2596542 $\mathrm{T}$ allele was dominantly significantly associated with decreased HCC risk development in chronic hepatitis $\mathrm{C}$ patients (adjusted $\mathrm{p}=0.04, \mathrm{OR}=0.55,95 \% \mathrm{CI}=[0.31$ 0.92]).We did not find a statistical significant association between rs9275572 in HLA and the risk of HCV-related HCC. However, rs9275572 A showed a significant association with increased risk of chronic hepatitis $\mathrm{C}$ susceptibility (Adjusted $\mathrm{p}=0.013$, $\mathrm{OR}=1.761,95 \% \mathrm{CI}=[1.126-2.754]$ ).

Conclusions: These results suggest that rs2596542 in MICA polymorphisms could affect the risk HCC among chronic hepatitis $\mathrm{C}$ patients, and rs9275572 in HLA may be associated with increased risk of chronic hepatitis $\mathrm{C}$ susceptibility. However, these genetic variants warrant further research for their clinical validity and utility in an independent population.

\section{PH-06 The FIND Specimen Bank: A Resource for the Development of Diagnostic Tools}

\section{A. Albertini, A. Mantsoki, C. Hoogland, S. Ongarello}

\section{FIND, Geneva, Switzerland}

Statement of the Problem: The sooner a patient is correctly diagnosed, the quicker treatment or appropriate care can be initiated. This year, over 3.8 billion infections in low-and middle-income countries (LMICs) are at risk of no/misdiagnosis. In order to meet the current and future needs of the scientific community, access to well-characterized, high-quality biospecimens is critical for the development of new technologies to improve patient care.

Proposed Solution: FIND manages collections of wellcharacterized disease samples in the fields of hepatitis $C$ virus, malaria, tuberculosis, and fever to support commercial and academic researchers in the development or evaluation of new and existing tools to improve diagnosis of infectious diseases in LMICs. The FIND Specimen Bank hosts over 400,000 specimens originating from over 40 sites, and over 25,000 patients. Specimen types include whole blood, serum, plasma, urine, sputum, saliva, DNA, bacterial samples, PAXgene tubes, and culture isolates. Specimen characterizations using microscopy, bacterial culture, molecular tests, antigen detection tests, and antibody detection tests depend on the disease. All specimens are collected at qualified clinics mostly located in LMICs, under a protocol approved by an ethical review board and with informed consent from patients meeting the inclusion criteria for specimen collection. FIND strictly follows good clinical practice in obtaining and processing specimens collected specifically for product development purposes, or as a complement to clinical trial activities.

In the last two years, FIND delivered over 16,000 aliquots to over 80 requesters worldwide. Specimen requests can be submitted via the FIND website. Each request is evaluated by a specimen bank review committee (SBRC) that approves specimen requests based on relevance to public health, applicability of the technology in high-burden countries, affordability of final product, and previous data or scientific evidence supporting the request. Following SBRC approval, samples are shipped worldwide. Requesters usually cover handling and shipping costs. All recipients are asked to report back on data linked to use of samples, and are encouraged to share results with the scientific community.

Conclusions: The FIND Specimen Bank aims to maximize access to geographically diverse biological samples for the international scientific community, resulting in life-saving diagnostic tools for multiple diseases.

\section{PH-07 How to Increase the Value of Swedish Sample Collections?}

E. Norén, M. Liljander, S. Eaker

Biobank Sweden, Jönköping, Sweden 
Background: Sweden has good conditions for medical research including human biological samples. There are national quality registers, national health data registers, biobanks with millions of stored samples, and a national system of personal registration numbers which makes it possible to link information from different sources after an approved ethical vetting.

The largest sample collections, which consist of samples collected within healthcare and stored in the county councils' biobanks for the purpose of diagnostics and treatment (approximately $95 \%$ of all stored samples), can be used in medical research after an approved ethical vetting, consent from the individual donor in accordance with ethical vetting, and an approved biobank application.

The primary purpose of the project was to investigate how the value of samples collected within healthcare can increase as a resource for medical research. The long-term goal is to enable the development of new knowledge about diseases for the purpose to improve the public health.

Methods: Experiences and comments were gathered during the autumn of 2018, through roundtable discussions as well as a survey. The respondents were personnel from the hospitals' laboratory medicine divisions (pathology, microbiology, and chemistry), local biobanks, biobank centers in health care regions, and scientists.

Results: It was emphasized that stored samples are a necessary tool for medical research to improve knowledge of diseases and how to treat them. However, based on the discussions, there are limitations mainly concerning access to and information about stored samples. Suggestions to increase the value of stored samples were: dedicated personnel categories within biobanks, visualization of sample collections, search ability of individual samples, improved information about the quality of individual samples, facilitated consent from sample donors, and efficient routines to pick out samples from storage.

Conclusions: The main conclusion is that stored samples are of great value and that the value may be further increased through several actions. To receive full impact of those actions in the context of a common biobank structure and common goals, the inclusion of the concerned professional occupations as well as a national collaboration between county councils, universities, and industry is of the utmost importance.

\section{PH-08 Genomics and Biobanking in African Settings: The Strides Made and Status Quo}

\section{Kezimbira}

\section{Immunology, Makerere University, Kampala, Uganda}

Introduction: Genomics wields the power to define and revolutionize modern-day precision medicine due to its highresolution power applied in the diagnosis and treatment of infectious diseases compared to the empirical methods especially in the research world. Genomics has witnessed a rampant evolution in the developed world as a consequence of the biobanks and biorepositories. Fully fledged bio-banks are very critical and a one-stop shop for preserving, processing, accessing, and long-term storage of all kinds of biological specimens; some initiatives have been introduced in some resource-limited countries in terms of infrastructure and capacity building, especially in Africa like the H3Africa and B3Africa. However, these efforts have been limited to very few African countries, leaving the majority unaccounted for.
Methods: We applied the Preferred Reporting Items for Systematic Reviews and Meta-Analyses guiding principle to examine the strides that have been taken to introduce genomics and biobanks in Africa using the PubMed NCBI database.

Results: We discovered very limited evidence of efforts and biobanking infrastructure to support the fast-evolving genomics discipline in African settings.

Conclusion: We propose various approaches and models that when adopted will realize the increased outreach of bio-banks, biorepositories, and genomics, consequently checking on the burden of infectious diseases on the African continent.

\section{PH-09 Use of Biorepository Facilities to Establish Familial Cancers Detection Program in Upper Egypt}

\author{
N. K. Kordy ${ }^{1}$, R. Mohamed ${ }^{1}$, S. Ezzat ${ }^{2,1}$ \\ ${ }^{1}$ Research, Shifaa Al-Orman Hospital, Luxor, Egypt, ${ }^{2}$ National \\ Liver Institute, Shibin, Egypt
}

Background: Since the establishment of Luxor biobank in October 2017, one of its objectives is to make use of the stored samples in different research studies. Therefore, Luxor biobank takes the initiative to establish a familial cancers detection program integrated with the biobank program. Familial cancers prevalence is not known among the Egyptian population. Moreover, the population in Upper Egypt is known for high consanguinity which might increase the prevalence of familial cancers. Identifying cancer driver genes involved in familial cancers in Egyptian population will help in appropriate detection of individuals with a higher risk of devolving cancer, and applying an appropriate early detection program. Our aim is to recruit cases with suspected familial cancers and detect genetic alterations associated with those cases.

Methods: The study was conducted at Shifaa Al Orman Oncology Hospital (SOH) located in Luxor, Egypt. The study was approved by the hospital institutional review board. The main malignancies that we focused on were breast, ovarian, colorectal, pancreatic, prostate, and uterine cancers. A researcher elicits a patient who is fullfilling the criteria for genetic risk determination according to the NCCN Guidelines and obtains their consent form. Additionally a questionnaire is distributed to determine participants' medical and familial cancer history to assess familial cancer risk. After blood collection, the blood is processed and stored. A database is developed to document sample information. Once this information is collected, a risk assessment model is used to evaluate risk and drawing pedigrees for patients with suspected BRCA. We are planning to perform genetic analysis using next-generation sequencing technology to analyze 30 genes associated with increased risk to develop familial cancers.

Results: A number of 354 were recruited patients to be involved in this ongoing observational study. We recruited 93 patients with positive family history and 46 patients with suspected BRCA-related Breast and Ovarian Syndrome.

Conclusion: Luxor biobank is considered the first in Upper Egypt to incorporate a biobank program along with a detection of familial cancer program. Familial cancer detection is lacking in our country, and there is no available information regarding the magnitude compared to western countries. Therefore, this study is essential to measure the magnitude of the problem and to enact future counseling programs and early detection programs as appropriate. 


\section{PH-11 Implementation of a Biliary Fluid Samples Collection in Navarrabiomed Biobank for Translational Research in Pancreatic Carcinoma and Biliary Obstructions}

I. Gil-Aldea ${ }^{2,1}$, B. Rodríguez ${ }^{2}$, P. I. Ana ${ }^{2}$, M. Cañas ${ }^{2}$, U. M. Jesus ${ }^{3,1}$

${ }^{1}$ IdiSNA, Pamplona, Spain, ${ }^{2}$ Biobank, Navarrabiomed, Complejo Hospitalario de Navarra, UPNA, Pamplona, Navarra, Spain, ${ }^{3}$ Gastroenterology Department, Complejo Hospitalario de Navarra, Pamplona, Navarra, Spain

Statement of the Problem: Clinicians of Complejo Hospitalario de Navarra (Spain) willing to carry on translational research in early diagnosis and prognosis biomarkers for pancreatic carcinoma and biliary obstructions decided to start collecting bile in Navarrabiomed Biobank, as well as blood derivatives and tissue. Patients were recruited in the hospital facilities, endoscopies, and operations rooms. Patients undergoing either a diagnostic endoscopic procedure or a surgical procedure from either benign or malignant clinical diagnoses in Complejo Hospitalario de Navarra were informed and requested to participate in our study by signing the inform consent form approved by our institutional review board. Patients provided samples for the study of biomarkers and/or remaining samples to be stored in the biobank for future related studies. Bile was a special type of sample to collect and handle. We reviewed the available bibliography at that moment for biliary fluid sampling procedures. There were not many papers and strong evidence about the best specific procedure to apply on bile samples could not be concluded.

Proposed Solution: We decided to start our prospective collection with our own sampling and datasheet standard operating procedure (SOP) to guarantee sample quality (our biobank is ISO 9001 certified since 2012). For developing our own SOP, we took into account not only the bibliographic data, but also some internal assays we did in our biobank in order to improve the biliary fluid sampling procedure. Bile samples were sent to the biobank on ice in a special cooler, centrifugated for 10 minutes $\left(+4^{\circ} \mathrm{C}\right)$ at $3500 \mathrm{~g}(4410 \mathrm{rpm})$ and stored in aliquots at $-80^{\circ} \mathrm{C}$ in the biobank facilities. All the processes are performed in less than two hours.

Conclusions: We started our samples collection in February, 2015. Since then, we have recruited 327 patients and handled 4,544 samples, including bile, bile cell pellet, whole blood, serum, plasma, buffy coat, and frozen and paraffin-embedded tissue. In all, 488 bile and serum specimens have been distributed and 678 slides and 288 automated immunohistochemistry assays have been performed in our biobank for several projects that obtained national and regional (Navarra) public funding. In addition, a translational research group for the study of pancreatic carcinoma and biliary obstructions has been created across all the projects, including clinicians (gastroenterologists, oncologists, and pathologists), fellows, and predoctoral and postdoctoral basic researchers in the field, as well as our biobank.

\section{PH-12 Success Story: How Does a Biobank Contribute to the High-Quality Research on Clinical Use of Biomarkers}

M. Karlikova, R. Kucera, O. Topolcan, J. Kinkorova

Department of Immunochemistry, University Hospital Pilsen and Faculty of Medicine in Pilsen, Pilsen, Czechia

Background: The Biobank Pilsen is a hospital-based biobank, established in 2017, although it was fully equipped al- ready in 2015. It has been collecting blood serum and plasma specimens from patients with various malignancies. At present there are more than 4,000 coded sample tubes stored at $-80^{\circ} \mathrm{C}$ in the biobank. Stored specimens are available for research projects running mainly in the University Hospital Pilsen and Faculty of Medicine in Pilsen, but also in other academical institutions.

Methods and Results: During the last three years, several research projects were succesfully accomplished with the help of the biobank specimens. We point out the four most important ones:

1) Testing and implementation of prostate health index: the aim was to assume, from the measurements of one serum biomarker, the diagnosis of prostate tumor, tumor agressiveness, and therapy decision.

2) Testing of serum PIVKA II as a new biomarker in liver carcinoma; the combination of AFP, CA 19-9, and PIVKA II helps to determine the presumptive histological type of tumor, together with its agressiveness

3) Testing of serum insulin-like growth factor- 1 as an additional tumor marker for various types of carcinoma, providing the information about tumor agressiveness.

4) Testing of plasma matrix metalloproteinases and their tissue inhibitors as biomarkers for the cancer invasiveness.

Conclusions: Biobank specimens are crucial for the research in oncology. In addition to the prospective studies, they allow to test the performance of new biomarkers on "old" samples from patients whose outcome is already known. However, it is crucial to have information about the specific biomarker stability. In our laboratory, we deal with this issue as well.

\section{PH-13 Preliminary Results of Cohort Biobank Development in Yogyakarta, Indonesia}

J. Fachiroh ${ }^{1}$, R. Wardani ${ }^{2}$, F. T. Pramatasari ${ }^{3}$, A. B. Hartopo ${ }^{4}$, F. Dewi ${ }^{5}$

${ }^{1}$ Department of Histology and Cell Biology, Faculty of Medicine, Public Health and Nursing UGM, Yogyakarta, Indonesia, ${ }^{2}$ HDSS, Faculty of Medicine, Public Health and Nursing UGM, Sleman, Yogyakarta, Indonesia, ${ }^{3}$ Biobank Unit, Faculty of Medicine, Public Health and Nursing UGM, Sleman, Yogyakarta, Indonesia, ${ }^{4}$ Department of Cardiology, Faculty of Medicine, Public Health and Nursing UGM, Sleman, Yogyakarta, Indonesia, ${ }^{5}$ Department of Health Promotion, Faculty of Medicine, Public Health and Nursing UGM, Sleman, Yogyakarta, Indonesia

Sleman Health Demographic Surveillance Survey (HDSS) is a population-based cohort operated for the last five years. This cohort surveys $\sim 5,000$ households representing $\sim 20,000$ populations from rural-urban areas of Sleman municipality in Yogyakarta Special Province, Indonesia. To increase the value of the cohort, a preliminary study to recruit biospecimen was done, in collaboration with biobank.

Through four subdistrict municipalities, Sleman HDSS management invited $\geq 18$ year old members of cohort, 60 per district. A survey was operated in each of the subdistrict municipal offices, two days each, three sessions/day, 10 respondents/ session. Each session was started with general health and study information provided through presentation and leaflet closed by question and answer ( $\sim 20$ mins), continued with individual consenting ( $\sim 10 \mathrm{mins})$. Parallel health examinations were done including weight, height, as well as waist and upper arm circumverence measurements, blood pressure measurement, one-time blood glucose and total cholesterol tested, and electrocardigraph 
(ECG) examination. Ten mililiters $(10 \mathrm{~mL})$ of blood from the arm was sampled and sent to the biobank. Formal report of the clinical condition was given directly except the ECG test, with onsite consultation to general practicioner. Before leaving, an exit survey was done to learn opinion of the subjects related to the process. Reimbursement for local transport cost was also given (IDR 50,000=USD 3.33). Each session also invited observer from the HDSS management.

One hundred and thirty-six (136) of 240 subjects came to the survey site, with participation rate ranging from $40-70 \%$ for each district. The $\mathrm{M} / \mathrm{F}$ ratio was $1 / 1.4$, age range $18-62$ years old, the majority with nine years compulsary education (89.94\%). Subjects felt satistied with the process, including information and service given prior and during survey, health examination, blood sampling process, ECG, and financial compensation. Subjects were happy to receive direct benefit through health examination and free consultation. On the other hand, several points needed to be considered, including time required per subject (70-80 minutes), low participation rate, extensive manpower recruited for the study, knowledge of sampling technician for the quality of blood samples upon temporary storage and transport. Overall, this study needs to reshape its strategy to optimize the resources, while allowing conditional adjustment with characteristics of the target population.

\section{PH-14 Metadata Analysis of Consent Outcome at Two Academic Medical Centers Participating in a Cancer Biobank}

K. Lepping ${ }^{1}$, N. King ${ }^{1}, \mathrm{~S} \mathrm{Li}^{2}$, C. Fisher ${ }^{2}$, K. Furman ${ }^{2}$, T. Schulte ${ }^{1}$

${ }^{1}$ Lombardi Comprehensive Cancer Center, Georgetown University, Washington, District of Columbia, United States, ${ }^{2}$ MedStar Health Research Institute, Washington, District of Columbia, United States

Background: Global biobanking projects further scientific knowledge by establishing infrastructures that streamline cancer research. Large-scale analysis is made possible through the comprehensive knowledge gained from a repository of both biospecimens and clinical data. Research on patient biobank participation rates reveals varied results with some studies reporting consent rates as low as $30 \%$, while others report rates above $95 \%$. This discrepancy may be explained by situational variables in the consenting process and patient population differences. Minimal risk of and the altruistic nature of biobanking leads to general patient support; however, growing societal interest in how centers share patients' DNA may be changing public and patient perceptions of biobanking. This research seeks to understand what factors impact cancer biobank consent rates through the examination of descriptive data from an academic-linked health system in Washington, DC.

Methods: Patients undergoing surgical procedures at two medical centers $(\mathrm{N}=703)$ were approached for participation in a cancer biobank from 2018 to 2019. Participation involves a onetime intervention to collect tissue, blood, and clinical data. Consent status, age, sex, tumor localization, size of tumor, and consent time and location were recorded and analyzed. Statistical differences were determined using chi-square tests.

Results: Over $78 \%$ of patients approached for consent agreed to participate, and over $95 \%$ of patients agreed to donate all biospecimens and associated clinical data to the biorepository. There was no statistically significant association between patient age, sex, tumor localization, tumor size, and/or location of approach on consent status within our patient pop- ulation. Patients were just as likely to consent to the study regardless of whether they were approached hours or days before their surgical procedure.

Conclusions: While we did not find a statistically significant impact of age, sex, consent location, or disease history on consent outcome, we concluded that the majority of patients approached for enrollment were amenable to participating in a biobanking study. Further studies that extend analysis to patient demographics, disease history, and patient perceptions are needed to better understand a patient's decision to participate. Understanding the intrinsic and extrinsic motivators for participation are integral toward building sustainable models for maintaining a diverse enrollment in biobanks.

\section{PH-15 Satellite Lab Concept to Reduce Processing Turnaround Time}

G. J. Welch ${ }^{1}$, A. Bridgeman ${ }^{2}$, R. Root ${ }^{1}$, T. Maran ${ }^{3}$, C. Mikorski ${ }^{1}$, H. Miller ${ }^{3}$, S. Bakkum-Hansen ${ }^{1}$, M. Yrjo ${ }^{1}$, M. Cicek ${ }^{1,4}$

${ }^{1}$ Biospecimens Accessioning and Processing Laboratory, Mayo Clinic, Rochester, Minnesota, United States, ${ }^{2}$ Advanced Diagnostics Laboratory, Mayo Clinic, Rochester, Minnesota, United States, ${ }^{3}$ Management Engineering and Internal Consulting, Mayo Clinic, Rochester, Minnesota, United States, ${ }^{4}$ Department of Laboratory Medicine and Pathology, Mayo Clinic, Rochester, Minnesota, United States

Background: The mission of the Mayo Clinic Biorepositories Program is to support Mayo Clinic Principal Investigator research initiatives through state-of-the-art biospecimen processing and storage, with a focus on quality and service. To meet industry and study standards, processing turnaround times (TAT) of less than two hours are required. Due to a multitude of variables, two-hour processing TAT was unattainable with the current model. The goal was to meet the protocol requirements for sample processing TAT.

Methods: Key stakeholders with the common goal of this project included research group principal investigators and program managers, biorepository program directors, an operations manager, lab supervisors, a quality coordinator and project manager, and a health systems engineer from the department of Management Engineering and Internal Consulting. The requirements from the research group, current process flows, and available TAT data were collated and analyzed.

Results: BAP baseline measurements for processing TAT were measured over a three-week timeframe. The sample size was 79 collections. On average automated processing TAT was 8.2 hours while manual processing TAT was 5.7 hours. Factors contributing to a higher TAT for processing of samples included sample collection workflows, the location of the collection site and proximity to the processing lab, the extended travel route for sample movement, and updates to the regulatory guidelines for processing samples within a two-hour timeframe. The project team implemented a satellite lab as well as a number of other interventions to decrease TAT for sample processing and increase customer satisfaction. Re-measurement of sample TAT at one month post-go-live demonstrated a decrease in average manual processing to 1.6 hours. Four-month post-implementation TAT showed an even further decrease to 1.2 hours.

Conclusion: The largest contributor to savings in TAT was the time spent in transporting the samples from the collection site to the lab. This transportation time was significantly decreased due to the satellite lab implementation resulting in an overall decrease in TAT. Other interventions were implemented 
as routine workflows within the satellite lab. BAP Leadership and key stakeholders confirmed improved customer service. The BAP Satellite Lab at the NW Clinic may serve as a model for future quality improvements.

\section{PH-16 Establishment of a Biobank in Kenya Medical Research Institute (KEMRI)}

J. M. Mwangi, L. K. Kanyara

Sample Management and Biobanking Division, KEMRI, Nairobi, Nairobi, Kenya

Rapid advancements in biomedical research demands quick access to biological samples and cell lines that meet desirable quality specifications for different applications. Collecting relevant information about the biospecimens together with proper isolation, purification, and processing of biospecimens and storing them in a well-structured biobank is becoming a more relevant strategy in assuring a rapid laboratory response in dealing with a range of diseases. Kenya Medical Research Institute (KEMRI) is composed of several research laboratories that conduct both basic biomedical and clinical research. There is need to have a well-structured system for data, biospecimen and bio-molecular pooling, storage, and sharing. Studies on molecular epidemiology, metabolomics, proteomics, translational medicine, drug discovery, therapeutic targets, and biomarkers need bioresources that should be sourced from a qualified biobank. In Kenya, KEMRI is known to be the key medical research facility and many researchers intend to collaborate with the institution for bioresources. There is need to scale up operations in KEMRI to allow biobanking infrastructure for safe storage and use of biospecimens and bio-molecular resources. The establishment of a biobank will aid in bridging this gap and enable scientists to gain unique insight into the genetic and environmental causal factors associated with a wide range of debilitating diseases, providing vital information. KEMRI hosts a wide range of experienced researchers who collect data and biological materials nationally, a nationally recognized ethical review board, access to utilities with backup generators, and hosts reference and accredited laboratories. Challenges of establishing a biobank in Kenya include lack of funding, infrastructure, trained staff, intellectual property issues, and appropriate legal and ethical regulations. Key steps in establishing a biobank include developing regulatory documents and a quality control program, proper data management, training personnel, collaborating with established biobanks, and stakeholder involvement. The potential benefits of biobanks in developing countries is largely unexplored, despite many decades of medical research and increasing world-class laboratories. Biobanks in low-and-middle-income countries such as Kenya will offer huge potential to address high disease burden in these countries.

\section{PH-17 Strategies for Fast and Efficient Access to Bioresources of National Biobank of Korea}

N. Kim, S. Shim, B. Ji, H. Kil, M. Lee, J. Jeon, D. Lee

Division Biobank for Health Sciences, National Institute of Health, Cheongju-si, South Korea

National Biobank of Korea (NBK) supported research by offering human bioresources and related epidemiological, clinical, or genetic data. We provided 539,114 vials of biospecimens, 788 sets of epidemiological and clinical data, and
557 sets of genetic data for 734 research projects from 2003 to 2018. Since KBN opened one-stop distribution service that allows researchers to retrieve human bioresources and apply for distribution online in 2014, the number of the NBK-supported research projects has been increasing dramatically. Therefore, we considered to develop a fast distribution system enabling to reduce the required period for distribution. We have established working bank systems comprising ready-to-use DNA, serum/ plasma, and data. DNA samples of 20,000 individuals that are stored at the NBK underwent the quality control (QC) procedure. The DNA samples that passed the QC were aliquoted into tubes labeled with a 2D barcode reacked in 96-format. We have been also loading serum and plasma in the automated working bank system since 2018 targeting to store 111,087 vials by 2019. The data working bank holds 42 pre-made epidemiological and genomic data sets which researchers have requested frequently. After opening of the data working bank, the required period for distribution was decreased by 11.7 days on average. Our fast distribution systems will allow more users to access and receive bioresources of NBK more quickly.

\section{PH-18 Building a Cancer Research and Precision Medicine Biobank for NCI Cancer Moonshot Research Programs}

L. Agrawal ${ }^{1}$, P. Guan ${ }^{1}$, A. Rao ${ }^{1}$, E. Casas-Silva ${ }^{1}$, V. Gopalakrishnan ${ }^{1}$, H. Ellis ${ }^{1}$, C. Weil ${ }^{1}$, J. Suh ${ }^{2}$, S. McDermott ${ }^{2}$, M. Jensen ${ }^{2}$, J. McClean ${ }^{2}$, N. Madero ${ }^{2}$, J. Wanyiri ${ }^{2}$, M. Williams ${ }^{2}$, H. Moore ${ }^{1}$

${ }^{1}$ National Cancer Institute, Rockville, Maryland, United States, ${ }^{2}$ Leidos Biomedical Research Inc., Frederick, Maryland, United States

Statement of Problem: The U.S. National Cancer Institute's (NCI) Cancer Moonshot program aims to accelerate research in key areas identified by a Blue Ribbon Panel of experts. The NCI Cancer Moonshot Biobank will establish a national biobank to facilitate cancer research and precision medicine through longitudinal biospecimen collections from diverse participant populations.

Proposed Solution: The Cancer Moonshot Biobank will collect longitudinal biospecimens (matched tissue and blood) from over 1,000 participants who are receiving standard of care cancer treatment in their communities. The initial phase of this study will include colorectal, lung, prostate, and gastroesophageal cancers, melanoma, multiple myeloma, and acute myeloid leukemia. National Community Oncology Research Program sites will enroll participants into the study and serve as biospecimen source sites (BSS). A BSS coordinator will provide coordination, site start up, and administrative assistance. Participants will be enrolled using the Oncology Patient Enrollment Network linked to the Interactive Web Response System with subsequent transfer to Medidata Rave, which will capture clinical and biospecimen data. The program will utilize electronic consent and a single IRB will provide oversight. A next-generation sequencing assay of participants' tumor tissue will be performed in a CLIA setting and a report shared with providers and participants. A central biobank, the Biospecimen Core Resource, will operate under standardized procedures. An interactive Participant and Provider Engagement Portal will facilitate communications and participation in the program. A portion of eligible fresh tumor specimens will be used to establish patient-derived models for future research needs. The biospecimens and extensive accompanying clinical and biospecimen processing data, along with molecular profiling data, will be made available to 
researchers via a National Institutes of Health data repository under controlled access.

Conclusion: The Cancer Moonshot Biobank will be a networked resource of longitudinal biospecimens that will help accelerate our understanding of cancer and facilitate new treatment and prevention opportunities, which can reduce the burden of cancer in all Americans and the health disparities that currently exist.

\section{PH-19 Recent Activities of Korea Gynecologic Cancer Bank}

H. Kwon, J. Kim, H. Cho, J. Choi

Gangnam Severance Hospital, Seoul, Korea

Background: Human-derived specimens emerged as important resources for basic and transitional research in gynecologic cancer which is critical in accelerating development of molecular-based diagnostics and therapeutics for precision medicine. In spite of the expanding needs for adequate humanderived specimens in gynecologic cancer studies, there was no well-established biobank for gynecologic cancer. Biobanking requires effective and efficient management of not only tissue sampling and storage, but also systematic management of bioinformatics and distribution of utilize high-quality research materials. We would like to introduce a biobank experiences for biospecimens of common gynecologic cancer

Methods: Human specimen and data stored in the bank target primary gynecologic cancer cell line, tissue, serum, plasma, lymphocyte, urine, saliva, and ascites. Specimen extraction was administered starting from 2012, and it was administered before or during the treatment using the low-invasive method with the patients, agreement. Specimen quality and quantity was identified by classifying specimen by cancer type, acquired year, and characteristic. Amount of specimen that was lent and distributed was verified, and published papers that were studied with these specimens were checked. Moreover, the institutions that signed a work agreement with the Bank for collection of the specimen and academic interaction were verified

Results: Currently, specimen and data in the bank numbers total of 62,898. Starting from May 2012 to October 2019, 21,548 serum, 17,403 plasma, 4,791 lymphocyte, 99 whole blood, 3,486 frozen tissue, 6,791 ascites, 36 HOSE, 4,940 urine, 350 saliva, 1,200 thin-prep, 440 cervicovaginal fluid, eight TMAs, and 1,806 paraffin block units were stored.

Conclusion: Resources of gynecologic cancer bank is continuing to grow steadily since 2012 , and quality resources are being developed through proper management. As such, these resources are utilized to publish a number of outstanding research papers. Likewise, request for distribution and lending for new research is increasing. It is necessary to continue to acquire and manage resources continually to establish the mechanism and the treatment method of the gynecologic cancer that are not confirmed to this point. It is judged that it would be necessary to provide resources actively according to the fair and appropriate procedure of the related research institutions and academic community.

\section{PH-20 Exosomal mi-RNA: Role in Diagnosis and} Prognosis of Pancreatic Cancer in Vietnam

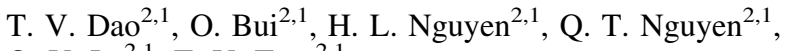
Q. V. Le ${ }^{2,1}$, T. V. Tran ${ }^{2,1}$

${ }^{1}$ National Cancer Institute, Thanh Tri, Hanoi, Vietnam,

${ }^{2}$ National Cancer Hospital, Hanoi, Vietnam
Background: Pancreatic cancer (PC) is commonly associated with high risk of mortality and this can be explained by lack of methods that allow physicians to diagnose it early. Exosomas carrying tumor-sensitivity mi-RNA are currently researched and raising more concern as new potential markers are applied in prophylaxis and treatment of PC worldwide and in Vietnam as well.

Methods: To investigate the application of exosomal miRNAs in diagnosis of PC, we collected 200 blood samples from PC patients and 200 other samples from patients diagnosed with liver cancer at the three biggest hospitals in Northern Vietnam (National Cancer Hospital, Viet Duc Hospital, and Bach Mai Hospital) from December 2016 to December 2019. All 400 samples would be analyzed to identify the quantity of mi-RNAs concentration, sensitivity, and area under curve. We chose five mi-RNAs that showed probability of diagnosis to further research on their potential of prognosis. To investigate the application of exosomal mi-RNAs in prognosis of PC, we chose 30 patients whose diagnoses were advanced carcinoma pancreatic cancer. All of them then received GEMOX regimen. We collected blood samples every three cycles and at the end of treatment. Five mi-RNAs mentioned above would be extracted from samples and quantified to set up an association with the outcomes of patients (pathology, stage of disease).

Results: From 400 blood samples analyzed, we defined five mi-RNAs had potential value of diagnosis to be further researched as prognostic factors: mi-17, mi-21, mi-155, and mi2190. Until now, there were 24 patients enrolled and underwent GEMOX regimen, all samples had been collected and stored for further analyzing.

Conclusion: Exosomal mi-RNAs are promising methods in diagnosis and prognosis of pancreatic cancer but further research needs carrying out to firmly certify the association as well as to confirm the effectiveness when applying in clinical practice.

\section{PH-21 Application and Exploration of Folic Acid Receptor Detection of Early NSCLC Based on Pulmonary Nodules}

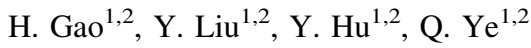

${ }^{1}$ Biobank, Nanjing Drum Tower Hospital, Nanjing, Jiangsu, China, ${ }^{2}$ Department of Pathology, Biobank of Nanjing Drum Hospital, Nanjing, Jiangsu, China

Background: Lung cancer represents the third most common cancer in China. Non-small cell lung cancer (NSCLC) is the most common type. NSCLC patients were often diagnosed on clinical imaging performed with symptoms. Pulmonary nodules in imaging examination without symptoms face a dilemma for surgical treatment or conservative treatment.

Methods: We selected plasma and paraffin samples (FFPE) of 389 patients with pulmonary nodules who have undergone surgery from Biobank of Nanjing Drum Tower Hospital and retrospectively analyzed the expression of FOLR1 in the plasma to evaluate the method of detecting FOLR1 positive cells in peripheral blood for NSCLC. According to the results of FOLR1 in peripheral blood, 206 patients pathologically diagnosed as early-stage NSCLC were divided into two groups: Group A (FOLR1 positive) and Group B (FOLR1 negative). Pathomorphological differences between the two groups were compared, including nodule size, type, stage, differentiation, and other indicators that reflect malignant degree and metastasis. The ICH expressions of FOLR1, ki-67 (proliferation related), TTF1 (differentiation degree related), and Napsin A (metastasis and invasion related) were evaluated in the tumor tissues. 
Results: The sensitivity and specificity of detecting FOLR1 in peripheral blood were $74.3 \%$ and $72.9 \%$, respectively. There were significant differences in classifications (adenocarcinoma in situ and invasive adenocarcinoma), stage (Stage 0 and I), and nodule size $(\mathrm{d} \leq 1 \mathrm{~cm}$ and $\mathrm{d}>1 \mathrm{~cm})$ between the two groups $(\mathrm{p}=0.013, \mathrm{p}=0.014, \mathrm{p}=0.041)$. Meanwhile, ki-67, TTF1, and Napsin A immunohistochemical scores in group B were significantly higher than those in group A $(p=0.035)$. IHC of our samples also suggested a high combined positive rate of $92.3 \%$ of MTHF and D2TYMS. Furthermore, MTHF and D2TYMS were closely related to DNA methylation, and the methylation of RASSF1A and SHOX2 showed a positive rate of $72.3 \%$ for NSCLC in this paper.

Conclusion: Detection of FORL1 positive cells in peripheral blood can be a good non-invasive screening method for NSCLC. Patients with FORL1 negative in peripheral blood have a poor prognosis and should undergo surgery in time, while patients with positive peripheral blood have better prognosis. This method can also be used as a supplementary surgical indications of pulmonary nodules. Methylation of RASSF1A and SHOX2 can also be used as detection targets of peripheral blood, alveolar lavage fluid, and pulmonary puncture cells.

\section{PH-22 Role of Korea Prostate Bank}

\section{J. Lee}

Urology, Catholic University Seoul St. Mary's Hospital, Seoul, Korea

Introduction: With the development of living standards of the people, the incidence of prostate disease has an increasing tendency. In 2009 , there were $8.9 \%$ prostate patients in Korea, which is the third most common type of cancer among men, statistics from the Korea Health Ministry have showed. The representative prostate diseases are prostatitis, BPH, and prostate cancer. Prostate disease research has been hampered by limited access to large numbers of human prostate tissue and biological samples with associated clinical and outcome data. We introduce the rapidly experience of a Korea prostate bank system and our nationwide prostate bank network.

Materials and Methods: The prostate bank holds specimens from patients with prostate cancer and benign prostate hyperplasia. The bank holds LN2 Tanks, below deep freezers, refrigerators to store the specimens in a high-quality state. It also possesses a patented cutting instrument developed by itself, virtual slide systems, and tissue micro array equipment. There are also centrifuges to process specimens, equipment to microdissect frozen tissue types, and also equipment to micro-dissect paraffin fixation tissue types. All the specimens have been barcodes to prevent patient exposure and to effectively manage the specimens. All the data at the bank are thoroughly managed by the Case Report Form (CRF) and the prostate bank online database program. The CRF database records over 50 types of clinical data and information to aid the investigator in his studies.

Results: The bank possesses about 80,000 specimens of gDNA, glass slides, frozen tissues, paraffin blocks, plasma, serum, and urine and parcels out specimens within the country and abroad. Each specimen was acquired after informed consent agreements with the patients. Due to these specimens, researchers have published in 30 scientific journals. Therefore, the bank contributes to research development by possessing a numerous amount of various, high-quality samples.

Conclusion: The goal of the prostate bank is to provide high-quality, well-characterized tissue samples from a large cohort of prostate disease cases, with associated clinical followup information. The clinical data will need to be updated at regular intervals. We think that if other countries like the U.S. and those in Europe, Africa, and Asia share the data, the prostate disease research will be more developed than now.

\section{PH-23 Biobanking in Low-Income Countries as Basis for Biomedical Science Development}

\section{G. Hartl ${ }^{1}$, A. Kaprin ${ }^{2}$, S. Petrov ${ }^{3}$, E. Hutter ${ }^{1}$, K. Sargsyan ${ }^{1}$ \\ ${ }^{1}$ Med Uni Graz, Graz, Austria, ${ }^{2}$ National Medical Research Radiological Centre of the Ministry of Health of the Russian Federation, Moscow, Russian Federation, ${ }^{3}$ Tsyb Medical Radiological Research Centre, National Medical Research Radiological Centre of the Ministry of Health of the Russian Federation, Obninsk, Russian Federation}

In the past decades, biomedical science has undergone rapid and thorough progress and has-along with and pushed by fastly evolving high-tech developments like artificial intelligencefundamentally changed also the field of biobanking, its working processes, and also its expected impact. On the other hand, there is strong evidence that the developments of biobanks and in biobanking along with the integral systematic examination of biospecimens and associated medical data have led to a much better understanding of diseases and disease prevention and has fostered also the development of new diagnoses and therapies.

There is a strong link between a solid investment in biobanking and the rapid increase of outstanding and ongoing achievements in biomedical science, which can be witnessed especially in many of the high-income countries with high funding rates for biomedical research and biobanks.

Having trained and supported more than 160 professionals from over 35 countries working in the construction, development, and/or process of a biobank, we at International Biobanking and Education at the medical university of Graz have experienced the challenges new biobanks and biobankers are confronted with in their drive for establishing a solid basis for biomedical research. Yet, we have also experienced a tremendous potential within biobanking, particularly for low-income countries with an often less-established and slowly progressing research infrastructure. Thus, in low-performing countries, investments and trainings in biobanks and biobanking have the potential to create a solid and credible data and knowledge basis, providing access to quality-defined samples and data and in turn driving forward further research at high quality and speed level.

\section{PH-24 Biobanking Spotlight on Europe, Middle-East, and Africa}

N. Afifi ${ }^{2}$, S. V. Anisimov ${ }^{6}$, R. Aquilar-Quesada ${ }^{7}$, J. Kinkorova $^{5}$, S. Nassimbwa ${ }^{8}$, S. Marrs ${ }^{3}$, Z. Kozlakidis ${ }^{1}$, A. Parry-Jones ${ }^{4}$

${ }^{1}$ Laboratory Services and Biobanking, IARC/WHO, Lyon, France, ${ }^{2}$ Qatar Biobank, Doha, Qatar, ${ }^{3}$ Haemotec, Pretoria, South Africa, ${ }^{4}$ Cardiff University, Wales Cancer Bank, Cardiff, United Kingdom, ${ }^{5}$ University Hospital in Pilsen and Charles University, Faculty of Medicine in Pilsen, Pilsen, Czechia, ${ }^{6}$ National BioService, St Petersburg, Russian Federation, ${ }^{7}$ Andalusian Public Health System Biobank, Granada, Spain, ${ }^{8}$ MRC/UVRI and LSHTM Uganda Research Unit,

Entebbe, Uganda 
Introduction: A regional ambassador program was initiated by ISBER in December 2018 to help the organization better understand local/national issues and requirements and to help expand the reach of the work. One of these regions is the EMEA region, comprising Europe, the Middle East, and Africa-a very large geographical area with diverse populations, needs, and challenges. The regional ambassadors were selected by a dedicated ISBER committee and are considered the regional thought-leaders in biobanking within their regions.

In this paper, an attempt has been made to highlight the challenges and achievements that remain at the top of the agenda for the ISBER-EMEA region. The ISBER regional ambassadors have highlighted key important/burning issues within their respective geographical regions working in conjunction with the EMEA Director-At-Large.

Methodology: Each ambassador provided representative information on three areas of general interest. These were:

1) The existing challenges in sharing samples and data, and whether there is an expectation that the challenges will be addressed in some way, locally or internationally. for the region.

2) The biobanks' impact assessment: how biobanks in the region are expected to deliver impactful work and how that is measured.

3) The two highest-priority issues in their respective region with regards to biobanking.

Results: The existing challenges were highlighted for the sharing of data and samples. These are not necessarily new, but demonstrate that slow progress has been made in addressing those identified some time ago. The ISBER ambassadors highlighted the value of networking and collaborations as a key in overcoming such issues. Furthermore, the biobanks' impact assessment presented an equally diverse picture, with the scientific impact being largely measurable, however lacking to denote the full impact delivered by the biobanks. The highest-priority issues identified had to do with the ethical and legal framework, education and training, the standardisation of practices, the certification and accreditation needs, and governance structures.

\section{PH-25 Genemap-Africa Biobanking Transformation and Future Directions}

O. G. Oluwole, C. Oosterwyk, S. Adadey, K. Mnika, A. Yalcouye, E. Wonkam, N. Manyisa, K. Popel, M. Jonas, M. Tutuka, V. Nembaware, A. Wonkam

Department of Pathology, Division of Human Genetics, Faculty of Health Sciences, University of Cape Town, Cape Town, South Africa

Background: Genemap Africa is one of the largest indigenous research initiatives conceptualized to study the -omics science of genetic diseases among Africans. Presently having collaborations in 10 African countries and over 8,000 different types of biospecimen in its care.

Statement of the Problem: However, in this context, the implementation of a laboratory information management system (LIMS) was limited. From a technical point of view, it was difficult to monitor all the activities from the recruitment sites across Africa. Biobanking management was the initial challenge that was confronted in the project. Typically, the project uses blood, DNA, RNA, cell-lines, and tissues. Before introducing the computerized laboratory management system, we recorded some damages to biospecimen.
Proposed Solution: We described, herein, Genemap semiautomated LIMs that enable the laboratory managers to connect the physical laboratory with the activities happening on the recruitment sites, onto the data capturing using a barcoding system, sampling (using an explicit unique identifier), experiments monitoring, shipping, storage, and data annotation. We introduced a standard operating procedure that combined preanalytical annotation and quality stratification, outlined to ensure that biological samples are collected adequately, and the appropriate kits are being used for the preservation, especially where there is electricity shortage, and to exercise timeous extractions before storing the specimen accordingly in the $-20^{\circ} \mathrm{C}$ or $-80^{\circ} \mathrm{C}$ ultra-low temperature freezer. Importantly, staff were trained on the shipping procedures.

Conclusion: Research approval rate and efficiency have improved significantly by more than $80 \%$ through the semiautomated Genemap LIMS. The quality check on over 2,000 biospecimens in this year using standard procedures showed that fewer than 20 samples have low qualities. In conclusion, the future direction, therefore, is to develop a meta-data platform, in the form of an "App" where synchronised data and reports can be archived and accessed electronically.

\section{PH-26 Enabling Efficient Discovery and Exchange of Specimens to Support and Accelerate Research Across the Pacific Northwest}

R. Galbraith ${ }^{1}$, A. Berry ${ }^{3}$, J. Buckner ${ }^{2}$, C. Drescher ${ }^{3}$, D. Galipeau ${ }^{4}$, B. Kostelecky ${ }^{1}$, G. Marchesini ${ }^{2}$, T. Rieckmann ${ }^{1}$, C. Rusinaru ${ }^{5}$, K. Smith ${ }^{6}$, A. Talhouk ${ }^{7,8}$, E. Holland ${ }^{1}$

${ }^{1}$ Fred Hutchinson Cancer Research Center, Seattle, Washington, United States, ${ }^{2}$ Benaroya Research Institute, Seattle, Washington, United States, ${ }^{3}$ Swedish Cancer Institute, Seattle, Washington, United States, ${ }^{4}$ Knight Cancer Institute, Oregon Health Sciences University, Portland, Oregon, United States, ${ }^{5}$ Legacy Research Institute, Legacy Health Systems, Portland, Oregon, United States, ${ }^{6} N W$ Bio, UW Medicine, Seattle, Washington, United States, ${ }^{7}$ University of British Columbia, Vancouver, British Columbia, Canada, ${ }^{8}$ BC Cancer Agency, Vancouver, British Columbia, Canada

Problem Statement: Among many challenges faced by the translational research community, the ability to find and access human-derived specimens is common and can lead to research bottlenecks and delays. Many investigators procure specimens through only one or a small number of sources. Each potential source has a limited volume of patients/participants from which specimens may be procured, and availability at a single source in a hospital setting is limited to the demographics of their patient population. Seemingly paradoxically, groups with large numbers of banked specimens often struggle to increase awareness and use of their collected specimens, which can lead to limited return on investment for high infrastructure costs.

Proposed Solution: To address the above-mentioned challenges, we formed the Specimen Acquisition Network (SAN). Our primary goal is to increase awareness of and access to highquality human-derived specimens, thereby speeding the pace of research across the Pacific Northwest and improving return on investment for biobanking efforts. Our network brings together seven key partners (Fred Hutch, BC Cancer, Benaroya Research Institute, Legacy Health Systems, University of Washington, Oregon Health Sciences University, and Swedish Cancer Institute) in support of this mission.

Representatives from each SAN organization have been meeting since early 2019 to develop the vision and implementation 
strategy for this network. This works included development of the SAN operating framework and a clear scope of work for the coordinating center. The strategy for the network is to ease the burden on researchers by creating clear processes and systems for procurement across each partner site. The SAN coordinating center, based at Fred Hutch, provides quality customer service and a streamlined, efficient method for identifying and requesting specimens from network partners.

Conclusions: In January 2020, the SAN officially launched its Coordinating Center and began to facilitate the exchange of specimens to drive research forward. In addition, the SAN is working closely with the Cascadia Data Discovery Initiative (CDDI) to inform development of a platform that will enable investigators to search across sites to discover specimen cohorts that would meet their research needs. This will speed the discovery process and further the network goal of increasing awareness of valuable research resources across the Pacific Northwest.

\section{PH-27 Macquarie University Neurodegenerative Disease Biobank}

S. Furlong, E. Cachia, S. D’Silva, K. Deang, J. Atkin, R. Chung, D. Rowe, V. Jacob

Macquarie University, New South Wales, Australia

Motor neuron disease (MND) is a fatal neurodegenerative disease characterised by progressive loss of motor neurons, which control muscle movement and function. From symptom onset, patients typically die within two to five years and every year in Australia around 800 people are diagnosed with this disease. Currently, there is no therapy to reverse the effects of the disease or to prevent its progression.

In 2013, Macquarie University's Centre for MND research and Macquarie Neurology clinic joined forces to establish the Neurodegenerative Disease Biobank. MND patients and controls are invited to participate in the biobank, by donating blood, urine, hair, and skin biopsies. Extensive clinical data are collected from each participant. Participants are also invited to complete an online environmental and lifestyle questionnaire. Data are stored within a custom-built database system.

This resource has grown rapidly with approximately 900 participants, 2,2200 collections, and 37,000 sample aliquots. As one of the largest MND biobanks in the world, with a collection of well-characterized samples and extensive clinical and lifestyle data, it is an extremely valuable resource to investigate MND pathogenesis and treatment strategies.

The biobank attained NSW Health biobank certification in 2019. Access to biobank resources is obtained via a biobank access committee and a cost recovery fee applies. Since 2013, 19 research projects have availed of this resource. Multiple biomarker projects study longitudinal collections of plasma, serum, urine, and hair, to identify biomarkers. Fibroblasts grown in the lab from skin biopsies are used to investigate protein degradation. DNA samples are used to identify new MND genes and to develop new models for investigating MND. This team has been involved in most of the major MND genetic advances identified to date.

Project MiNE is international collaborative effort in which whole genome sequencing is carried out to identify rare gene variations. SALSA is a national research effort into the sporadic onset of MND. Environment projects search for evidence of neurotoxin and pollutant exposure in plasma samples from sporadic patients living within geographical clusters of MND.
This biobank is an excellent example of a medium-sized biobank with a collection of high-quality samples, interdigitated with extensive clinical records that can support numerous complex research projects to make major advances in understanding of complex disease.

\section{PH-28 The Center for Prostate Disease Research Biospecimen Bank and Multicenter National Database: Integrating Biospecimen and Clinical Data for Precision Medicine}

S. Tan ${ }^{1,2}$, L. Ravindranath ${ }^{1,2}$, D. Young ${ }^{1,2}$, A. Ali $^{1,2}$, Y. Song ${ }^{1,2}$, B. Pannell ${ }^{1,2}$, D. Nousome ${ }^{1,2}$,

S. Elsamanoudi ${ }^{1,2}$, I. A. Sesterhenn ${ }^{3}$, T. Sreenath ${ }^{1,2}$, J. Cullen ${ }^{1,2}$, I. L. Rosner ${ }^{2,4}$, G. Petrovics ${ }^{1,2}$

${ }^{1}$ Henry Jackson Foundation for the Advancement of Military Medicine, Bethesda, Maryland, United States, ${ }^{2}$ Center for Prostate Disease Research, Department of Surgery, Uniformed Services University of the Health Sciences, and the Walter Reed National Military Medical Center, Bethesda, Maryland, United States, ${ }^{3}$ Joint Pathology Center, Silver Spring, Maryland, United States, ${ }^{4}$ John P. Murtha Cancer Center, Walter Reed National Military Medical Center, Bethesda, Maryland, United States

The Center for Prostate Disease Research is a research program established to improve the early detection, prognosis, and treatment of prostate cancer (PCa) by integrating basic and clinical research. It comprises the Clinical Research Program, located at the Walter Reed National Military Medical Center (WRNMMC), the Biospecimen Bank, the Centralized Multicenter National Database ("the Database"), and the Translational Research Program. Quality control measures of the Biospecimen Bank are achieved by accreditation from College of American Pathologists.

Biospecimens, including urine, serum, and tissue, are collected at WRNMMC from consented patients under approval from the institutional review boards (IRB) of the WRNMMC and Uniformed Services University. A partnership with the Joint Pathology Center has ensured quality processing and histopathologic characterization of tissue specimens. The Database has longitudinally collected demographic, clinical, pathologic, treatment, and quality of life outcomes data from a racially diverse military cohort with equal access to healthcare. In compliance with Health Insurance Portability and Accountability Act law, biospecimens are deidentified, while clinical and pathologic data are stripped of patient health identifiers and anonymized. Best practices were established to link the BioBank and the Database under approved IRB protocols.

Since 1998, over 29,700 patients consented data, and over 7,500 patients consented both specimens and data for research use. Approximately 200,000 specimens, which include frozen tissue slides and blocks; FFPE sections of whole-mounted prostates; purified DNA samples; and aliquots of serum, plasma, and urine were collected. Through collaborative efforts of a multidisciplinary team of clinicians, health-care professionals, pathologists, scientists, epidemiologists, and bio-statisticians, these resources have supported over 100 peer-reviewed publications and over 20 grants, including the discovery of distinct genomic differences in $\mathrm{PCa}$ of racially diverse populations. Ongoing studies continue to accrue molecular, "omics," and pathologic image data from patient specimens.

We continue to enhance this unique resource by improving the quality of specimen collection, the organization of specimen storage, and the secure linkage and retrieval of data. To further 
enhance our translational research capabilities, we are building a repository for molecular, "omics," and digital image data with linkage to the biospecimens and the Database.

\section{Informatics \& Technology}

\section{PI-02 C3OD and OPTIK: Bioinformatic Tools to Help Evaluate Enrollment Impacts from Biobank Partnerships in an Underserved Community}

H. Kuo ${ }^{1}$, D. Pal Mudaranthakam ${ }^{2}$, P. Todd ${ }^{3}$, N. Malomo ${ }^{3}$, S. Madhusudhana ${ }^{3}$, H. Krebill ${ }^{1}$, M. Park ${ }^{4}$, L. Harlan-Williams ${ }^{5}$, M. S. Mayo ${ }^{2}$, J. Thompson ${ }^{2}$, B. Gajewski ${ }^{2}$, D. Koestler ${ }^{2}$

${ }^{1}$ Masonic Cancer Alliance, University of Kansas Cancer Center, Fairway, Kansas, United States, ${ }^{2}$ Department of Biostatistics and Data Science, University of Kansas Cancer Center, Kansas City, Kansas, United States, ${ }^{3}$ Oncology Research, Truman Medical Centers, Kansas City, Missouri, United States, ${ }^{4}$ Biospecimen Repository Core Facility, University of Kansas Cancer Center, Kansas City, Kansas, United States, ${ }^{5}$ Anatomy \& Cell Biology, University of Kansas Cancer Center, Kansas City, Kansas, United States

Statement of Problem: As the University of Kansas Cancer Center (KUCC) aims for the Comprehensive National Cancer Institute Cancer Center designation, partnerships and collaborations with local institutions are vital to reaching all communities throughout its catchment area, especially the underserved. Along with this, being able to quantify the extent of this reach with data and statistics is important so that evaluation of a program's effectiveness can be measured. For this instance, KUCC established a biobank partnership with a local safety-net hospital, Truman Medical Centers (TMC), which provides biobank donation opportunities to underserved patients. Data management resources and tools would be required to get a sense of how this partnership impacted biobank participation in the local underserved community.

Proposed Solution: KUCC's Biostatistics and Informatics Shared Resource (BISR) has developed tools that can support this evaluation, the first being the Curated Cancer Clinical Outcomes Database (C3OD), which can pull electronic data from the EMR system and link it to biospecimen inventory data (housed in OpenSpecimen). This allowed the biobank staff to extract zip codes from previously enrolled patients, as this was not being annotated before. The second BISR resource to be used was the data visualization team called OPTIK (Organize and Prioritize Trends to Inform KUCC). Underlying OPTIK has utilized the Tableau software to create a geographical visualization of biobank enrollments between TMC and KUCC using local zip codes. This provided an improved way of evaluating enrollment changes by plotting them on a local area map.

Conclusion: Advanced cancer centers must not only form partnerships to extend programs and treatments to the local community, but they also must develop a means to quantify how these collaborations make a difference. In this case, KUCC was able to form a key biobank partnership with a local safetynet hospital, TMC, while also using tools so that KUCC could understand how biobank opportunities and enrollments were being impacted in the surrounding area. This helps guide KUCC towards its goal of serving its catchment area. The combined use of C3OD and OPTIK, translational data warehouse initiatives, was able to quantify how biobank enrollments from an underserved population were impacted. For some Kansas City Metropolitan zip codes, biobank enrollment increase ranged from $25 \%$ to $141 \%$ through the TMC and KUCC partnership.

\section{PI-03 Development and Application of a Utility System in Platform-Based Biobank in General Hospital}

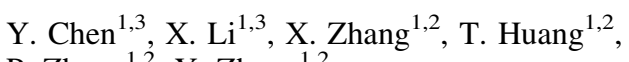
P. Zhang ${ }^{1,2}$, Y. Zhang ${ }^{1,2}$

${ }^{I}$ Research Center, Beijing Friendship Hospital, Capital Medical University, Beijing, China, ${ }^{2}$ National Clinical Research Center of Digestive Diseases, Beijing, China, ${ }^{3}$ Beijing Clinical Medical Institute, Beijing, China

Background: Biobanking has recently become an emerging field of academic and commercial interest in China. An increasing number of institutions have established their own biobanks at different levels of administration. One common problem is that a biobank is too large in quantity yet too low in utility. In order to solve this problem, and to realize the transition from high-quality storage to high-efficiency usage, we developed our own "Utility System of Clinical Data and Biobank Resource of Beijing Friendship Hospital, Capital Medical University (CMU)."

Methods: The Utility System was connected to FreezerPro $^{\circledR}$, our currently used sample management system, as well as Hospital information system in real-time. Standard PREanalytical Code (SPREAC), sample processing, current status, anonymous clinical data, and other critical information are integrated into the system. It was designed under the browser/server framework. By using different modules, it can fulfill different functions such as detailed description, advanced searching, online application, and bank list checking. Our User Interface design was inspired by leading business-tocustomer ecommerce sites.

Results: We designed four modules: module 1: Detailed quantified description, module 2: Advanced personalized searching, module 3: Precise limited application, and module 4: Strict graded management. The frequency of apply were significant improved right after the release of our system, from 41 in the first half of 2014 to 77 in the second half. This Utility System has been running for more than two years now. It has 165 registered users, information of 91 projects, and nearly 350,000 samples. As of September 2019, a total of 243 orders were completed by this system. The proportion of orders issued through the Utility System increased continuously, from $34.26 \%$ in the second half of 2017 to $98.25 \%$ in the first half of 2019.

Conclusions: The Utility System is convenient for users to search and apply online. It is convenient for medical administrators to obtain a full picture of their working projects. It has been widely used in our hospital, providing scientists with great convenience, facilitating clinical sample apply, and increasing usage rate in our biobank.

\section{PI-04 Monitoring the Impact of Extraction Methods on cfDNA Quality}

E. Viering, E. Graf

Agilent Technologies, Waldbronn, Germany

Background: Cell-free DNA (cfDNA) plays an emerging role as a source for potential new biomarkers. However, cfDNA still represents a demanding sample type due to restricted abundance and complex fragment size distribution. Yield and quality of cfDNA can vary drastically depending on pre-analytical treatment. Standardizing and monitoring pre-analytical parameters, including cfDNA extraction, is inevitable to achieve best possible sample quality. 
Method: Nine different extraction kits tailored for cfDNA extraction were used for comparison. Three independent plasma samples were processed with each kit in parallel and subsequently analyzed in six replicates with an electrophoretic assay suitable for cfDNA analysis. The assay offers total DNA quantification, and automatically assigns a region for cfDNA separated from high-molecular-weight (HMW) DNA by size. Using region analysis, the concentration of cfDNA apart from HMW DNA is evaluated by the software. The quality score $\%$ cfDNA is provided as additional quality parameter, determining the percent cfDNA of total DNA in presence of HWM. This set of quality parameters adjusted to the needs of cfDNA characterization was applied to compare the extraction kits.

Result: The cfDNA extraction kits varied in rate of yield and their size selection capacity to remove HMW DNA. In general, each kit afforded either superior yield or high selectivity towards cfDNA. High yield may be crucial for plasma with low cfDNA content, yet if the plasma is expected to contain considerable amounts of HMW DNA, the selectivity towards cfDNA might be favored.

Conclusion: The choice for the most suitable cfDNA extraction kit depends on the expected cfDNA concentration and the occurrence of HMW DNA. Even if no preference for a single kit could be established, the results present a straightforward way to monitor the impact of pre-analytical parameters on cfDNA yield and quality. Automatic evaluation of a quality score for cfDNA allows to define a threshold for objective sample quality assessment.

\section{PI-06 Development of Biobanking In-House Wireless Sensor Network for Temperature and Humidity Monitoring}

\section{K. Kanyara ${ }^{1,2}$}

${ }^{1}$ Sample Repository Management Facility, KEMRI, Nairobi, Kenya, ${ }^{2}$ Information Technology, Strathmore University, Nairobi, Kenya

A laboratory manager's greatest fear is to walk into their laboratory and find equipment, for instance, a freezer or another temperature-controlled unit, has failed and damaged vital medical samples, such as specimens or tissues. A recent example is the loss of critical samples used in research donated from deceased people with autism at McLean Hospital, a Harvard Research Hospital, in June 2012 due to a freezer failure which thawed 147 brains. Even though an alarm and monitoring system was put in place, it failed to notify staff of the freezer malfunction. A higher-level system of monitoring with built-in redundancy could have helped produce a better outcome; however, most are cost prohibitive for individual labs to install and maintain (Boother, 2013).

With the available monitoring systems in the market there is need for a laboratory wireless sensor network, a service that is supported by high data rates that are emerging in the market. A laboratory wireless sensor network is a network that is suitably used by laboratories to collect, store, and transmit data wirelessly.

Problem Statement: As discussed previously, existing laboratory monitoring systems aim to provide reliability and security of data. Some of these systems stand out in data collection by use of thermometers and sensors, and, in case of failure, set off alarms. However, all these systems are dependent on having to either manually input data or log the data using Wi-Fi to transmit data on a cloud-based platform.
Methods: The proposed laboratory wireless sensor network will employ a star topology. This is mainly because the LoRaWAN technology works best on a star topology and star topology employs high speed, reliability, maintainability, and is quite flexible. The sensor devices are to connect to the centrally located device, the LoRaWAN gateway. All the devices on the network connect to the gateway through a communication link.

Proposed Solution: Biobanking in-house laboratory wireless sensor network is a network-based system that aims to efficiently collect and ensure secure wireless data transmission from laboratory devices to a computer service, then transmit the notification of events to the users' mobile phones. The system will provide a secure and cost-effective solution compared to the commercially available networks.

\section{PI-07 Development of a Prognostic Gene Signature Based on an Immunogenomic Landscape Analysis of Bladder Cancer: A Bioinformatical Analysis Based on Biobank}

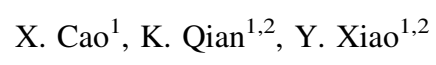

${ }^{1}$ Department of Biological Repositories, Zhongnan Hospital of Wuhan University, Wuhan, China, ${ }^{2}$ Human Genetics Resource Preservation Center of Hubei Province, Wuhan, China

Background: Tumor immunology and immunotherapy are one of the exciting areas in bladder cancer research. However, there has been no investigation to establish robust prognostic signatures based on an immunogenomic landscape analysis of bladder cancer. This study aimed to develop an immune-related genes signature to improve the prognostic predictions of bladder cancer.

Methods: We identified differentially expressed immunerelated genes (IRGs) based on TCGA database, and gained insights into the biological functions of IRGs using Metascape, then constructed a prognostic IRGs signature with LASSO Cox regression.

Results: Three hundred ninety-two differentially expressed IRGs were identified, including 215 up-regulated and 177 downregulated genes. Functional enrichment analysis revealed that these genes were enriched in inflammatory pathways, including in "regulation of signaling receptor activity," "cytokinecytokine receptor interaction," and "GPCR ligand binding." A prognostic six-IRGs signature was constructed, including AHNAK, OAS1, APOBEC3H, SCG2, CTSE, and KIR2DS4. The results of multivariable Cox regression and subgroup analysis revealed that six-IRGs signature was a clinically independent prognostic factor for bladder cancer patients. Further, we constructed a nomogram based on six-IRGs signature and other clinicopathological risk factors, and it performed well in predict patients' survival.

Conclusions: our research may provide a novel immunerelated genes signature to estimate prognosis for patient survival with bladder cancer, it might facilitate bladder cancer patients counselling and individualise management.

\section{PI-08 The Construction of Biobank Based on the Real-World Study}

C. Pu, Q. Zhang, W. Liu, Z. Wang, H. Huang, C. Liu

Dalian 6th People's Hospital, Dalian, China

Objective: To explore the strategies of constructing a clinical biobank based on the real-world study. 
Methods: Biospecimens have been collected since November 2014, as well as continuous construction of the biobank resources to match the implementation of real-world study. Related measurements adopted include standardized ethical management, quality control, automated data management, and follow-up management.

Results: Biospecimens and clinical data information were collected with high quality, according to standard SOPs. Sophisticated equipment and data automation management mode were used to ensure the protection of patient privacy and data security. At the same time, biospecimens and data information management system were improved gradually to fulfill the integration and sharing of biospecimen data resources.

Conclusions: Biorepositories are the key to translational medical research. Biospecimens collected in our biobank will link with clinical data information simultaneously, which increases the resource pool that can be applied in real world study.

\section{PI-09 Dashboards and Information Workflows Supporting Dissemination of the Russian Human Radiobiological Tissue Repository}

\author{
A. Alaoui ${ }^{1}$, D. Goerlitz ${ }^{1}$, S. Sokolova ${ }^{2}$, M. Zakharova ${ }^{2}$, \\ E. Kirillova ${ }^{2}$, C. Loffredo ${ }^{1}$ \\ ${ }^{1}$ Oncology, Georgetown University Medical Center, \\ Washington, District of Columbia, United States, ${ }^{2}$ Southern \\ Urals Biophysics Institute, Ozyorsk, Russian Federation
}

Information systems are critical to the support of human biorepositories. Increasingly, they are also key to the effective dissemination of information about such biobanks, including informing the scientific community of available biospecimens and the procedures to request access. Such a system has been developed to support the Russian Radiobiology Human Tissue Repository (RHTR), which was established in 1998 and is located in the Southern Urals Biophysics Institute of the Federal MedicalBiological Agency in Ozyorsk, Chelyabinsk Region, Russian Federation. RHTR is a repository of over 287,000 biospecimens from about 9,000 individuals collected from 1951-present. Biospecimens come from workers at the Mayak Production Association who were exposed to occupational internal alpha and/or external gamma radiation. The RHTR also houses collections of biospecimens from the offspring of Mayak PA workers, and from residents of Ozyorsk, who while never employed at the Mayak PA, have been exposed to elevated levels of radiation due to residence in the vicinity of the Mayak PA. The RHTR was established under a joint scientific research agreement between the governments of the Russian Federation and the United States in cooperation on research of radiation health effects for the purpose of minimizing the consequences of radioactive exposures. The biosamples collected by the RHTR are processed into a wide range of tissue types using standardized laboratory techniques and quality control practices; the data annotation includes complete dosimetric and exposure histories for Mayak PA workers, together with occupational, medical, and demographic data stored in electronic databases. The biological materials and their annotated data are available to investigators and have been shared with interested scientists worldwide via a website that allows users to search the catalog of biospecimens for materials of interest. Online application processes have been established to allow users to apply for and receive these materials. We here present the established methods and workflows to collect, store, and make available the biospecimen resources of the RHTR. We also present the developed dashboards and processes to query and request samples for research and collaboration purposes.

\section{PI-10 Federated IT infrastructure for Cancer Moonshot Biobank}

V. Gopalakrishnan ${ }^{1}$, P. Guan ${ }^{1}$, J. McClean ${ }^{2}$, N. Madero ${ }^{2}$, M. Jensen ${ }^{2}$, H. Ellis ${ }^{1}$, J. Suh ${ }^{2}$, S. McDermott ${ }^{2}$, E. Casas-Silva ${ }^{1}$, L. Agrawal ${ }^{1}$, C. Weil ${ }^{1}$, J. Wanyiri ${ }^{2}$, A. Rao ${ }^{1}$, M. Williams ${ }^{2}$, H. Moore ${ }^{1}$

${ }^{1}$ Biorepositories and Biospecimens Research Branch, National Cancer Institute, Rockville, Maryland, United States, ${ }^{2}$ Leidos

Biomedical Research, Inc., Rockville, Maryland, United States

Statement of Problem: There is an increasing need to collect high-quality biospecimens from diverse participants to support research that facilitates the understanding of complex genetic diseases such as cancer and develop more effective targeted therapies. Biobanking studies need robust information technology (IT) infrastructure to support the collection, storage, and distribution of biospecimens and associated data. However, lack of well-defined requirements, variability in systems, their interoperability, data models, and institutions involved are some of the challenges in setting up an IT framework to serve biobank needs.

Proposed Solution: NCI's Biorepositories and Biospecimens Research Branch has initiated the Cancer Moonshot Biobank (CMB) to collect longitudinal biospecimens from over a thousand cancer patients being treated with standardof-care therapies in medical institutions across the U.S. The biospecimens will be distributed to qualified researchers to accelerate progress in studying drug resistance and sensitivity. An integrated project team comprising experts in IT and bioinformatics was set up to define requirements, perform landscape analysis, identify systems, and coordinate the implementation of systems to serve the needs of the Biobank. CMB's IT infrastructure includes 1) an electronic consent application to consent patients interested in participating in the study; 2) a patient registration system to enroll consented patients; 3) a clinical data management system to collect participants' clinical and biospecimen data at the medical institutions; 4) a patient and provider engagement website to enable engagement with study participants including providing secure access to clinical test results; 5) a biorepository LIMS to track biospecimen processing, quality, storage, and distribution; 6) an online biospecimen catalog to display inventory of biospecimens available to researchers; and 7) a cloud-based data sharing platform to provide controlled access of CMB data to researchers. These systems will be integrated to ensure seamless transmission of data in order to improve both the providers' and participants' experience in the study and provide rapid access of high-quality data to researchers.

Conclusion: CMB's federated IT infrastructure is envisioned not only to support the collection of biospecimens and associated data, and its distribution to researchers, but also-in the spirit of the Cancer Moonshot-to provide a platform for direct engagement with patients and providers.

\section{Innovative Technology}

\section{PJ-01 Color Two-Dimensional Code}

J. Zhang

Hangzhou Biobank Biotechnology Co., Ltd., Hangzhou, Zhejiang Province, China 
The colored two-dimensional barcode is a matrix twodimensional barcode. The image of the matrix two-dimensional barcode is composed of several color blocks arranged in a rectangular array; the edge of the image is a circle of color blocks to form a positioning area, and the internal color block of the image constitutes an encoding area. The positioning area comprises an " $L$ "-type edge and a positioning edge relative to the "L"-type edge. By adding six colors to the original data structure of Datamatrix and forming a new color channel with black and white dichroism, the invention builds a colored twodimensional barcode, enhances the encoding capacity to three times of the original, and maintains maximum compatibility with the existing black and white Datamatrix encoding system. By introducing eight colors to construct a colored twodimensional barcode, the original one-byte data represented by a single color block is increased to three bytes, and the encoding capacity is increased to three times of the original one. That is, under the condition that the size of encoding matrix and recognition symbol is unchanged, the original two-dimensional barcodes encoding 12 bits of information can accommodate 36 bits of information, and the capacity of error-correcting barcodes is increased accordingly, which not only completely solves the problem of insufficient coding capacity of small-area two-dimensional barcodes, but also increases the reliability of two-dimensional barcode parsing.

\section{PJ-02 Increasing Process Stability and Sample Safety through Cryogenic Scanning of Biological Samples}

E. Gossla, L. Doms, J. Tämmerich

\section{Askion GmbH, Gera, Germany}

Statement of the Problem: Biobanks are frequently confronted with the challenge of transferring existing samples from other storage systems or registering them for this purpose while maintaining sample quality. Just as important as the storage temperature are the handling temperature and the processes for transferring sensitive biological materials. During the relocation of samples from other temperature ranges $\left(-20^{\circ} \mathrm{C},-80^{\circ} \mathrm{C}\right)$ but also from other liquid nitrogen storage systems, significant temperature fluctuations can occur during transport and scanning, which may impair the quality of the samples. If the scanning takes place at room temperature or a relatively high temperature compared to the storage temperature, the samples, racks, and tubes are warmed up. As a result, samples cannot be scanned correctly due to fogging of the barcodes and ice formation occurs during subsequent storage, which makes automated storage more problematic, especially with non-readable barcodes or frozen samples. To ensure sample quality, temperature fluctuations during transfer must also be avoided.

Proposed Solution: The development of a rack scanner that can be utilized at room temperature and also at cryogenic temperatures between $+24^{\circ} \mathrm{C}$ and $-150^{\circ} \mathrm{C}$ increases sample safety and improves the safe automated handling of samples. By scanning in a cooled environment, the temperature fluctuation of the samples can be significantly reduced compared to scanning at room temperature. In addition, condensation and subsequently the formation of ice is significantly reduced or prevented. The Askion ColdEye was developed as a stand-alone instrument and for the integration into automated LN storage system HS200. By developing the ColdEye it is now possible to capture bottom barcodes of various formats directly and reliably in a cold environment. Additionally, this enables the optical control of the storage process in a cold environment and can prevent picking errors due to frozen tubes by detecting the picking of adhered tubes by digital image processing and initiating automatic troubleshooting procedures.

Conclusions: ColdEye enables improved sample safety during transfer and storage processes. Due to the portable device, samples can be scanned in a cold environment (on dry ice or in a nitrogen-cooled work bench). In addition, it allows the scanning during automated sample handling at temperatures down to $-150^{\circ} \mathrm{C}$, which leads to a significantly increased safety in automated handling.

\section{PJ-03 Impact of New PBMC Isolation Tubes and the Use of a Liquid Handler on PBMC Cell Yields, Cell Viability, Work Throughput, and Hands-On Time}

\section{L. Aparicio}

\section{Clas Automation, Miami, Florida, United States}

Peripheral blood mononuclear cells (PBMC) are the cell population of choice for many clinical trials and clinical investigations. The standard method, density gradient centrifugation, for isolation of PBMC is a laborious and timeconsuming manual process and, thus, one of the most limiting factors for processing large numbers of patient specimens. Manual methods are difficult to standardize due to the number of variables involved in the generation of the gradient-diluted blood interface and harvesting of the PBMC band. Our study described herein focuses on the outcome of manual and automated strategies to 1) layer diluted blood over the density gradient and 2) harvest the PBMC band using a newly developed configuration of a $30-\mathrm{mL}$ tube with and without the use of a liquid handler. This $30-\mathrm{mL}$ tube consists of a lower and upper chamber connected by a narrow cylindrical segment that facilitates manual layering of diluted blood over the density gradient as well as generating a more concentrated PBMC band with minimal aspiration of the density gradient. Results from the manual and automated processes using the new isolation tubes were compared to the results of the predicate method, using standard 50-mL centrifugation tubes, based on total cell recovery and cell viability. Results showed greater than $95 \%$ mean total cell recovery compared to the manual method and mean cell viability greater than $91 \%$ while reducing granulocyte contamination by greater than $31 \%$ compared to values obtained by predicate method. This proposed strategy for automated and standardized method for preparing PBMC can significantly enhance the use of cell based-assay in clinical trials by improving overall quality and efficiency of the process as well as reducing labor cost.

\section{Repository Automation Technology}

\section{PK-01 Let Your Tubes Fly! A Modular Pneumatic Storage Network Delivering High-Quality Sample Access for Translational Research in China}

\section{P. K. Lomax}

\section{Marketing, TTP Labtech, Royston, United Kingdom}

In 2014 the Chinese government announced investment to develop five National Centres for Translational Research with the first of these planned in Shanghai across two sites at Jiao Tong University and Ruijin Hospital. Much emphasis has been placed on bringing people together to foster collaboration between different disciplines, such as engineering, clinical, and 
basic research with the common goal of driving innovation and more rapid development of medicines, therapies, devices, and diagnostics.

As the first of these centres planned its multi-disciplinary research facilities, it was clear that access to high-quality samples would be key to delivering world-class research. During the planning phase there was much debate between the research teams about the merits of a central biobank versus smaller local storage facilities and there were clear pros and cons for both approaches. The other challenge was the estimation of the sample storage capacity required as this had the potential to vary significantly as the centre evolved. Getting this wrong could mean that storage capacity could quickly run out or conversely that space and funding could be wasted on storage space that could remain empty for years.

In evaluating solutions for automated storage, the team became interested in the possibilities offered by TTP Labtech's arktic $-80^{\circ} \mathrm{C}$ storage systems and, in particular, the possibility to interlink them through a pneumatic transport system. After further consideration, the decision was made to proceed with discrete clusters of arktic modules for each of the different research teams. This meant that the storage units could be placed conveniently in different locations in the building and could be housed in standard laboratory space. The teams also planned for relatively short-term storage capacity projections, safe in the knowledge that capacity could be easily expanded later with the addition of extra arktic modules. Longer term there is also the option to interconnect the storage clusters if it is deemed advantageous to share samples across the research groups in the future. It was also realised that sample handling and workflow efficiency could be improved using remote access modules to transfer samples through pneumatic pipes directly between the laboratories and the storage facilities which were housed on separate floors of the building.

This poster provides an overview of the system implementation, workflows, and envisaged future enhancement.

\section{PK-02 Clearing the Air: Adjusting Practices to Meet Atmospheric Conditions with Automated Freezers}

L. Zhou ${ }^{1}$, J. Hawks ${ }^{2}$, J. Patterson ${ }^{3}$, N. Hartigan ${ }^{3}$, R. Filonzi ${ }^{3}$, N. Gabrael ${ }^{1}$, A. Yuksel ${ }^{1}$, D. R. Catchpoole ${ }^{1}$

${ }^{1}$ Kids Research, The Tumour Bank - CCRU, Westmead, New South Wales, Australia, ${ }^{2}$ Hamilton Storage Technologies, Franklin, Massachusetts, United States, ${ }^{3}$ Biostrategy, Melbourne, Victoria, Australia

Automated freezer technology currently relies on barcodes to identify, locate, and distribute sample tubes within an ultracold environment. However, build-up of ice crystals in the form of frost must be avoided; otherwise, incorrect or failed barcode reads will create inventory gaps, errors, and sample management inconsistency within the system. Dry air purging within the system aims to create low humidity conditions during sample entry into a $-80^{\circ} \mathrm{C}$ system. Despite this, when installing and commissioning a small automated freezer we encountered an unforeseen issue that the ambient humidity of the room where the freezer was located dramatically influenced frost build-up during the introduction of snap-frozen tubes into the freezer. Consequently, sample entry into an automated freezer was systematically reviewed and validated to adjust for this issue. The lower freezing temperature of alcohol-based solutions prevent ice formation. The interaction of different alcohols with plastics alters the strength of ice crystal anchoring to a surface. We therefore compared procedures where tubes were sprayed with various dilutions of alcohols, and mopped up with absorbent pads and/or brushing off the frost during the sample transferral procedure. Tubes were snap frozen in liquid nitrogen then stored in $\mathrm{a}-80^{\circ} \mathrm{C}$ manual freezer until all daily tubes had been processed. Twelve racks of tubes were sprayed lightly with $75 \%$ or $100 \%$ isopropanol prior to introduction into the automated freezer or were doused in $100 \%$ isopropanol prior to any freezer transfer event using either the manual or automated $-80^{\circ} \mathrm{C}$ freezer. The methods were evaluated quantitatively through regular daily systems audit results whilst photographs provided qualitative evidence. The room environment temperature and relative humidity were monitored throughout the experiments. The time management to attempt each step during each approach was also noted. We specifically noted that spraying snap-frozen tubes with $100 \%$ isopropanol early dramatically reduced frost build-up during subsequent sample transfer steps through the humid room to the automated freezer. Too much isopropanol, however, caused handling errors due to slippery surfaces. Isopropanol caused a film to form on some plastic which reflects differences in chemical reactivity between polycarbonate vs polypropylene which likely influenced frost build-up. Site-specific operating procedures need to be considered when installing new automated freezing systems.

\section{PK-03 DNA Traceability a Model of Automation in Qatar Biobank Laboratory}

M. Markovic Bordoski, E. Alkhayat, F. Qafoud, N. Afifi, S. Mohamed, T. Alhamad, S. Alresheq

\section{Qatar Biobank, Doha, Qatar}

Background: In Qatar Biobank (QBB), automatic robotic liquid handlers with integrated devices are replacing all critical procedural steps. Traceability of samples along with sample quality and accuracy can significantly decrease sample mismatch and discrepancies from specimen collection, processing, analysis, retrieval, data collection, and reporting.

Methods: To generate a traceable DNA sample with participant DNA fingerprinting and genotyping critical steps in the process are identified in the QBB laboratory. Estimation of DNA concentration is based on fluorometric protocol where manual and automated processes are in place and validation is performed for comparison. Normalization of DNA is performed with bidirectional communication between a laboratory information management system and an integrated robotic system where high-quality and quantity DNA is processed for the diverse forthcoming process. One of the downstream applications of normalized DNA in QBB is sample genotyping based on microarray technology; both manual and automation chip preparation protocols are introduced, and obtained results were plotted against each other during verification processes.

Results: The manual quantification process was performed in triplicate, using a two-point standard curve versus automated customized protocol where the eight-point standard curve was developed using the same positive controls for accuracy and precision estimation. Where both methods met the accuracy and precision criteria, a statistically significant difference was observed in the overall variance between triplicates. The same trend was observed during verification of manual and automated DNA normalization step. During microarray genotyping the overall plate genotyping call rate for automated preparation had less variability and dispersion of the data generated on a total eight plates.

Conclusions: Established automation systems are leading to better sample management and increasing sample throughput 
traceability is critical in obtaining and retrieving high-quality sample and data. However, each of those systems needed to be validated/verified and compared with the manual process, as during any breakdown manual is the immediate back-up ensuring uninterrupted workflow leading to preservation of each of the precious samples fulfilling the core role for every biobank.

\section{Repository Management}

\section{PL-01 The Establishment of GLMD Biobank and Quality Control System Provides a High-Quality Sample for the Prevention and Treatment of Glucolipid Metabolic Disease Scientific Research with Integrated Chinese and Western Medicine}

Y. Chen, W. Zhou

First Affiliated Hospital of Guangdong Pharmaceutical University, Guangzhou, China

Background: The incidence of glucolipid metabolic disease (GLMD), including dyslipidemia, nonalcoholic fatty liver, obesity, type 2 diabetes mellitus, and atherosclerotic cardiocerebrovascular disease and many other related similar diseases, is high, and it is a worldwide problem. In the future, biomarkers that can be used for early warning, diagnosis, clinical treatment, and prognosis evaluation of GLMD have been the focus and difficulty of research.

Methods: GLMD Biobank was established in accordance with the "Biobank Standards of China Medical Biotechnology Association" and ISBER standards. Diagnoses of GLMD was according to the "Technical Specification for Diagnosis and Treatment of Glucolipid Metabolic Disease (Danzhuo) Integrated Traditional Chinese and Western Medicine" issued by the Committee of Metabolic Diseases of the World Federation of Chinese Medicine Societies. The clinical samples of volunteers included plasma, blood cells, white blood cells, buffy coat, saliva, urine, etc. Sample management software was used to record sample information, clinical information, medications, TCM symptoms, laboratory tests (including blood sugar, blood lipids, and insulin, etc.), using the "Four diagnostic instruments of traditional Chinese Medicine" (Daosheng DS01-A) to store tongue and pulse. Regular random sampling was performed for quality control, such as RNA integrity and purity, sample location information, and sample clinical information.

Results: From establishment to August 1, 2019, the GLMD Biobank has stored 28,720 samples and 1,888 patients. The RNA stored in the blood cells, white blood cells, and buffy coat of the regular sampling test was relatively complete, and the sample was stored at $-80^{\circ} \mathrm{C}$ for more than three years without degradation.

Conclusions: The GLMD Biobank and quality control system were initially established to provide high-quality clinical sample resources for the prevention and treatment of GLMD scientific research by integrated Chinese and Western medicine.

\section{PL-02 Cost Recovery in the New Era of Biobanking}

\section{L. Wellman, L. M. Glenn, M. Clements, A. Gibbs}

Pathology \& Anatomy, Eastern Virginia Medical School, Norfolk, Virginia, United States

Statement of the Problem: It is widely acknowledged biobanks are a critical bridge in propelling scientific discovery in the new age of genetic research. Biobanks are essential as direct access to high-quality biospecimens is exceedingly difficult and while the ease of access a biobank provides to researchers is invaluable, many biobanks struggle to meet sustainable cost recovery. Most biorepositories lack secure long-term funding and may solely rely on institutional support. Developing a viable and potentially profitable infrastructure is key in ensuring biobanks remain a valuable asset to future scientific discoveries.

Proposed Solution: The Eastern Virginia Medical School Biorepository is a small biobank that houses over 75,000 human specimens from a wide array of anatomical sites and disease states, backed by a comprehensive clinical database. It is the only CAP-accredited biorepository at an academic health center in the state of Virginia and is only the second medical facility in the commonwealth to earn this distinction. One strategy we employ to subsidize costs is our fee-for-service assistance of investigator-initiated project-based consenting, procuring, processing, storage, and collection management of samples. In addition to our repository, we have an attached histology core, which provides technical research pathology services to academic, clinical, and scientific organizations. The core specializes in both microtomy and cryotomy as well as routine and specialized tissue processing, IHC, special staining, tissue microarray development, and slide scanning. The largest portion of our cost recovery comes from this core, vastly helping to aid in sustainability.

Conclusions: Cost recovery for biorepositories remains a challenge. We propose the utilization of fee-for-service options for investigator-initiated projects as well as a basic histology core can both expand your range of clientele as well as serve as financial infrastructure to support the longevity of your repository.

\section{PL-03 At What Cost? Analysis of Two Different Informed Consent Mechanisms Employed by a Cancer Biobank}

K. Carvalho ${ }^{1}$, B. Gali ${ }^{1,2}$, J. LeBlanc ${ }^{1}$, L. Matzke ${ }^{2}$, P. Watson ${ }^{1,2}$

${ }^{1}$ Tumour Tissue Repository, BC Cancer, Victoria, British

Columbia, Canada, ${ }^{2}$ Office of Biobank Education and

Research, University of British Columbia, Vancouver, British Columbia, Canada

Background: The Permission to Contact (PTC) platform is a useful mechanism to increase patient engagement and enrollment to support research. It provides researchers the ability to retrospectively access and complete consent for criteriaspecific cohorts of patients who have expressed interest in participating in research. Here, we evaluate the costs to compile a specific research cohort based on either utilizing a PTC platform to complete consent for specific cases or operating a prospective consenting approach for all cases.

Methods: For this study, we contacted two cohorts of breast cancer patients who enrolled in a PTC platform as far back as 10 years. We contacted these patients to obtain specific consent to access clinical FFPE tissue blocks and associated clinical data. We evaluated the rates of occurrence of each consent outcome (i.e., obtained, declined, unreachable, lost to followup, waiver of consent), as well as time spent and costs associated with each outcome. Costs were calculated based on the average salaries of staff involved. The time and costs to consent these cohorts of patients were calculated and compared to a control cohort of breast cancer patients who were prospectively enrolled in the Tumour Tissue Repository during the same timeframe. 
Results: Breast cancer patients with either carcinoma in situ (DCIS, $n=90)$ or triple negative breast cancer (TNBC, $n=161$ ) were enrolled in the PTC platform between 2007 and 2015. Consent was obtained from 48/90 DCIS patients and 102/161 TNBC patients. The total cost of using the PTC platform was \$29.01 and \$22.20 per DCIS and TNBC patient consent, respectively. In comparison, the total cost of prospectively consenting DCIS and TNBC patients through standard biobanking procedures was up to \$373.64 and \$231.44 per-patient consent, respectively.

Conclusions: This study provides a better understanding of the feasibility of obtaining informed consent from patients several years after enrolling in a PTC platform. By comparing this mechanism to compiling the same cohorts based on a prospective consent mechanism, we show that the utilization of the PTC platform is an efficient and cost-effective method of developing criteria-specific cohorts to support research. Further, the data generated by our analysis guides operational decision making for our biobank and this approach is effective in attaining biobank sustainability.

\section{PL-04 Current Technology for Temperature Monitoring}

A. Bonk

Coris Life Sciences Monitoring, Mamaroneck, New York, United States

Statement of the Problem: ISBER Best Practices specify monitoring and reporting of fixed-temperature storage units with an automated system that creates logs and generates audit trails and alarms. A common approach has been a site-based system requiring the maintenance of a server to store temperature data and generate alerts. Configuring and maintaining a server and its related security systems (firewall and remote access token system) requires considerable time and information technology (IT) expertise from biorepository personnel or the involvement of an external IT group.

Proposed Solution: Cloud-based systems can eliminate the need to maintain an on-site server while providing redundant storage and continuous access to sensor data, log files, and reports. Some cloud-based systems may require an onsite server to store readings before transmitting this data to the cloud, and this approach requires a similar level of expertise and maintenance as an onsite-based system. Cloud-based systems include sensors that are hardwired to a local "communications" module that wirelessly communicates with a local wireless "hub" that has Internet access. The hub connecting to the Internet may permit two-way communication, creating a vulnerability in the firewall and an attractive hacking target. IT groups may require a sensor hub to be on a dedicated Internet connection, which may include a cell phone data modem or a separate Internet hardwired connection, both of which would require an additional cost for the lab. Alternatively, technology exists that eliminates the possibility of hacking the network and also eliminates the need for and cost of an additional Internet network connection.

Conclusions: Onsite-based and some cloud-based automated temperature monitoring systems require the maintenance of a server and involves considerable effort and IT expertise for laboratory personnel. Cloud-based systems typically eliminate the need to maintain an onsite server but involve additional challenges regarding network security. Selection of specific environmental monitoring sensor modules and hubs that connect to the Internet will enable continuous access to temperature data and alert control and eliminates the need to involve the institutional IT group while maintaining the security of the network.

\section{PL-05 Large-Scale Sample De-Identification at the Intermountain Healthcare Biorepository}

C. Sainsbury, D. Wilcox, T. H. Shaw, B. Sharp,

P. Waite, D. K. Crockett

Biorepository, Intermountain Healthcare, Salt Lake City, Utah, United States

Background: Intermountain Healthcare's Biorepository contains approximately 12 million samples (slides and blocks) that date back to the 1960s. Each sample is currently labeled with a patient identifier. Intermountain Healthcare places a high priority on protecting patient privacy so a workflow needed to be developed to solve this problem.

Methods: In order to protect patient privacy, we decided to capture this information along with a picture of the original label, assign a new de-identified number to the sample, and then physically remove the protected health information from the sample. We believed that it would take approximately two years with multiple staff members to complete the project. Contingent staff members were hired for the duration of the project with the ultimate goal of hiring approximately 10 of them as a full-time employee at the end of the project to continue de-identifying the approximately 500,000 samples that are received into the Biorepository each year. A database application was built to capture the unique information contained on each sample and take a picture. Each staff member works in a team of two. This first person enters the original sample information into the database and takes the picture. A new de-identified unique number is assigned to the sample, and a barcoded label is generated. The sample is then passed to the second person. They scan the barcode to populate the information entered, verify all information is correct, and make any necessary corrections. They then remove all original labels contained on the sample and place the new barcoded label on the sample. Finally, we also implemented a database audit that looks for erroneous information and any duplicate samples entered. This audit helps us identify any samples that were mis-labeled that might have passed through our initial quality check.

Results: Since beginning this process in January 2019, we have de-identified over 780,000 samples accurately linked to over 300,000 surgical events with numbers growing daily. New standard operating procedures and workflows were developed to ensure quality throughout the entire process.

Conclusions: The implementation of this system has allowed us to safeguard patient privacy in a large-scale repository. The system ensures that the samples can only be re-identified by Intermountain Healthcare's Biorepository, which enables us to serve as the Honest Broker for this large integrated healthcare system.

\section{PL-06 Ensuring Sustainability of a Biorepository through Strategic Planning: Our Experience at the McCain GU BioBank}

A. L. Bolanos ${ }^{1,2}$, M. Kenk ${ }^{1,2}$, A. Bhimani ${ }^{1,2}$, K. Hersey ${ }^{1,2}$, N. Fleshner ${ }^{1,2}$, H. Wagner ${ }^{1,2}$, S. Paul ${ }^{1,2}$

${ }^{1}$ McCain GU BioBank, Toronto, Ontario, Canada, ${ }^{2}$ Surgical Oncology, Princess Margaret Cancer Centre - University

Health Network, Toronto, Ontario, Canada 
Background: Biobanks support clinical trials and research programs, providing a vital resource for precision medicine and playing a key role in accelerating research. The demand for high quality biospecimens increased considerably in the last decade and will continue to expand in the future. If so, why are many biobanks not sustainable? Sustainability affects biobanks of all sizes and ages. Being set up without an understanding of the costs and challenges associated with running and maintaining a biorepository, most biobanks struggle with maintaining funding and utilization rates. The McCain GU BioBank has developed and implemented a business plan to attain sustainability.

Methods: In order to utilize biospecimens efficiently and guarantee the capacity to endure as a biorepository, the McCain GU BioBank took into account its strengths as the largest biobank for blood, urine, and tissue samples for uro-oncological biospecimens and developed a business plan to address vital questions: How can we improve and maintain the infrastructure required to maximize capacity with high-quality samples? Are our utilization target rates realistic? Which biobanking services are needed in the changing market? General and biorepositoryspecific planning tools (e.g., SWOT analysis, market surveys) were used to develop a business plan focused on sustainability.

Results: The business plan development involved internal and external stakeholders. Initial assessments identified areas of need, including a detailed organizational chart, risk assessment, and a review of product/service costs. Clear marketing strategies, risk assessments, and budget were established. The business plan was implemented, serving as the strategic plan for future operations, providing a clear roadmap with milestones for growth and expansion. To better match biospecimen supply and demand and increase utilization, the biobank works with academic and non-academic researchers on numerous studies and clinical trials locally and internationally. A business plan allows us to anticipate trends within the market, enabling optimized resource allocation, increasing productivity, and expanding collaborations.

Conclusion: Biobank sustainability is critical for biorepositories globally. By utilizing its strengths efficiently, ensuring adaptability to economic and social changes, and increasing utilization, the McCain GU BioBank has established a sustainable plan for growth.

\section{PL-07 Maintaining Sample Relationship in De-Identifying Multi-Facility and Multi-Year Samples}

D. Wilcox, B. Sharp, T. H. Shaw, C. Sainsbury, P. Waite, D. K. Crockett

Biorepository, Intermountain Healthcare, South Salt Lake City, Utah, United States

Background: In biorepositories, paraffin blocks and associated aliquots are initially catalogued and labeled using thirdparty systems. Our organization utilizes systems that maintain discrete relationships between samples using three data elements from sample collection that delineates an "accession": 1) the year, 2) the facility or hospital, and 3) a numeric sequence that increments with each sample and resets at the beginning of each year. The relationship between blocks and resulting aliquots via accession is imperative to ensuring everything can be tracked back to their origins and ultimately back to the donor. However, as the need to safeguard protected health information increases, many repositories are de-identifying samples in order to mitigate privacy risks. It was determined that existing laboratory information management systems (LIMs) were not capable of simultaneously de-identifying samples while also maintaining the sample relationship. Our objective was to find a way to effectively de-identify all biorepository samples while maintaining the relationship between associated samples and the medical record from where the sample originated.

Methods: We introduced a relational database referred to as a "Linkage Database." The linkage database consists of two tables minimally: 1) An "accession" table to assign a unique numeric identifier for each distinct combination of data elements that make up the original accession and 2) a "sample" table that assigns a unique identifier for each sample and maintains the relationship back to the original accession using the accession unique identifier. By maintaining the data in this fashion, it is possible to label samples with identifiers that do not contain PHI directly.

Results: The linkage database was implemented in January 2019 and currently has over 780,000 samples accurately linked to over 300,000 accessions with numbers growing daily. Because each sample is assigned a unique identifier, data can be exported using the pattern of [ACCESSION_ID]-[SAMPLE_ID] as a unique name for each sample and then imported into LIMs appropriately.

Conclusions: The implementation of a linkage database enables repositories to successfully de-identify samples in their care while maintaining the relationship between samples and originating donor. Additionally, the linkage database serves as an intermediary between identifiable clinical data, de-identified samples, and LIMs.

\section{PL-08 Integration of Real-Time Data Validation into BIMS (OpenSpecimen) for Data Quality Control within the Victorian Cancer Biobank}

\author{
M. Ling ${ }^{1}$, S. Adiga ${ }^{2}$, W. $\mathrm{Ng}^{1}$ \\ ${ }^{1}$ Victorian Cancer Biobank, Melbourne, Victoria, Australia, \\ ${ }^{2}$ Krishagni Solutions Pvt Ltd, Pune, India
}

Statement of the Problem: The Victorian Cancer Biobank (VCB) is a consortium of five tissue banks from health precincts in Melbourne, with the Cancer Council Victoria as the lead agency. Tissue bank staff from all consortium sites manually annotate an extensive dataset into the Biobanking Information Management System (BIMS), OpenSpecimen. Currently, data quality checks are performed as required for projects or for reporting purposes to detect inconsistencies and missing values. These checks involve data extraction from OpenSpecimen and the output is analysed with Excel. Any inconsistencies or missing values are marked on the spreadsheet and sent to the relevant tissue bank site for correction in OpenSpecimen. This is an inefficient process and a more effective data quality control is required. To address this, VCB is collaborating with Krishagni Solutions to integrate real-time data validation into OpenSpecimen.

Proposed Solution: The OpenSpecimen data validation consists of edit checks to detect and correct errors at time of data entry and this can be applied to any data element within OpenSpecimen. This feature is similar to that utilised by cancer registries for data quality control. The edit checks are defined via JSON based on specified records and rules. Record is the level (for example, participant, visit, specimen) which the edit check is applied to. A rule is made up of three attributes: 1) when-specifies when the rule is applicable, 2) exprspecifies the check constraint that should be satisfied, and 3) description-describes the error message/constraint in simple text. Edit checks can be set up to detect and reject values which are either outside of normal range or due to impossible/unlikely data element combinations. If data entered fails the edit checks, 
an error message will display on the screen and data will not be saved until the error is corrected to satisfy the edit checks. An error report summary of each user can be generated routinely for performance evaluation and improvement.

Conclusions: Integration of data validation into BIMS with automated generation of user error report will provide VCB an efficient and effective management of data quality, enable implementation of ongoing data quality assurance, and complement other data quality control measures. This will enable VCB to align with data quality management best practices incorporating a continuous cycle of analysis, assessment, and improvement of data quality.

\section{PL-09 Mexico National Biobank Network: Sustainability}

C. M. Luna Aguirre ${ }^{1,2}$, A. G. Gámez Medina ${ }^{1,2}$, M. A. Campomanes Flores ${ }^{2}$, S. Bernal Silva, ${ }^{3,4}$, B. Illades Aguilar ${ }^{5}$, C. R. López Macías ${ }^{6}$, J. P. Reyes Grajeda ${ }^{7}$, G. Vizcaíno Tapia ${ }^{8}$, A. Ulloa Aguirre ${ }^{8}$, C. Ximénez García ${ }^{9}$, L. A. Herrera Montalvo ${ }^{10}$, L. M. Ruiz-Godoy-Rivera ${ }^{10}$, R. López Ridaura ${ }^{11}$, M. Lajous Loaeza ${ }^{12}$, H. A. Barrera-Saldaña ${ }^{1,13}$

${ }^{1}$ Biobank, Vitaxentrum, Monterrey, Nuevo León, Mexico, ${ }^{2}$ Biobank, Innovita, Monterrey, Mexico, ${ }^{3}$ Microbiology, Autonomous University of San Luis Potosí, San Luis Potosí, Mexico, ${ }^{4}$ Research Center for Health Sciences and Biomedicine, Autonomous University of San Luis Potosí, San Luis Potosí, Mexico, ${ }^{5}$ Guerrero Autonomous University, Chilpancingo, Mexico, ${ }^{6}$ Mexican Social Security Institute, Mexico City, Mexico, ${ }^{7}$ National Institute of Genomic Medicine, Mexico City, Mexico, ${ }^{8}$ National Institute of Medical Sciences and Nutrition Salvador Zubiran, Mexico City, Mexico, ${ }^{9}$ National Autonomous University of Mexico, Mexico City, Mexico, ${ }^{10}$ National Institute of Cancerology, Mexico City, Mexico, ${ }^{11}$ National Center for Preventive Programs and Disease Control, Mexico City, Mexico, ${ }^{12}$ National Institute of Public Health, Morelos, Mexico, ${ }^{13}$ Ambassador LATAM, ISBER, Monterrey, Mexico

Statement of the Problem: The Mexican population has an inadequate representation in R\&D of the world's pharmaceutical and diagnostic industries. We are promoting the creation of a federated network of institutional biobanks as supporters of these $R \& D$ efforts and working with them to become sustainable.

Proposed Solutions: The plan to become a federated network includes: operating as a consortium (MxBioBaN); inviting Innbiogem, SC to coordinate it; joining ISBER; adopting and harmonizing ISBER; developing a sustainability model; promoting the ISBER mission in Latin America; acquiring funds to support consortium growth; and achieving international visibility through collaborating with business partners (Mexican Innovita + Canadian TransHit BIO). A key challenge for success is to gather international evidence to promote national legislation that considers the fundamental legal, social, ethical, and regulatory bases capable of propeling biobanking activities in our country and that is alluring for the international bioindustries.

Conclusions: The establishment of biobank networks that bridge health care with translational research to foster diagnostic and therapeutic innovation will allow Mexican leading medical research institutions to contribute to the world's efforts to develop new diagnoses and treatments. Evolution into MxBioBaN has achieved the governmental recognition of institutionalized biobanks. Our National Council of Science and Technology provided seed funds for its launching that were matched by our fund raising campaign. Collaborating biobanks have started to procure high-quality biospecimens and their clinical data for internal or external (national and international) research projects.

\section{PL-10 Modernization of the Intermountain Healthcare Biorepository}

D. K. Crockett, C. Sainsbury, D. Wilcox, S. D. Firth, P. Waite, M. H. Cessna

Biorepository, Intermountain Healthcare, Salt Lake City, Utah, United States

Background: The Biorepository is a marquee asset for Intermountain Healthcare, its subsidiaries, and commercial partners. This research archive comprises approximately 12 million samples (pathology blocks and slides) collected across some 50 years. The sheer size of the collection combined with longitudinal health records, genealogy, and image and genetic data is a unique and distinguishing feature that increases in value exponentially. The Biorepository serves as our foundation of samples and data for precision medicine efforts across the system and helps to innovate and improve patient care.

Methods: From 2017 to 2018, Boston Consulting Group (BCG) consulted on the Intermountain Biorepository. BCG provided a thorough roadmap to aid in modernization efforts of the Biorepository. Among other things, this modernization is being done with the intent of increasing the speed at which sample information can be accessed and queried, increasing the number and size of projects that the Biorepository can undertake, and providing more governance and structure around the operations of the Biorepository.

Results: Strong executive support has resulted in a new modern facility with expanded shelf/freezer capability for sample storage. Appropriate staffing has been addressed with key skillsets such as: project managers, inventory technicians, biostatistics, bioengineering, genomics, data consultants, and research histology. Data integration efforts have developed query tools and electronic inventory. Digital slide scanning and large-scale sample de-identification is underway. Lastly, the overall governance structure has also been re-built. Historical sample volumes for the Biorepository were averaging 600 to 800 per year. In 2018, total samples shipped were >8,000. 2019 samples shipped year-to-date are $>23,000$.

Conclusions: The Biorepository has already begun this modernization effort, and the results are already demonstrating system value in de-identification, honest broker capabilities, increased storage space, increased capacity in scale, clouddeployed data and query tools, and moving Intermountain towards the promise of digital pathology. The wise investment to modernize the Biorepository will pay multiple dividends for decades to come. However, careful guidance and clear goals for growth are required. The success of the Biorepository will also depend, in part, on successful partnerships being established with customers both internal and external to Intermountain.

\section{PL-11 Building a Management System for Drug Clinical Trial Sub-Biobank-Biobank of the First Affiliated Hospital of Zhengzhou University}

Z. Zhu, H. Wang, J. Xie, Y. Liu, S. Zhao, X. Tian

Biobank Department, First Affiliated Hospital of Zhengzhou University, Zheng Zhou City, Henan Province, China 
Background: Biobank plays an indispensable role for medical science and public health. The biobank of the First Affiliated Hospital of Zhengzhou University was launched in 2017. It aims to provide the best service for the scientific research by collecting and storing the samples. Our bank also provides temporal data for testing services. It has seven subbiobanks including Drug Clinical Trial (DCT) Sub-biobank, Cancer Sub-biobank, Rare Disease Sub-biobank, and so on. However, establishing a perfect management system to ensure the quality of biological samples for biobank still confronts challenges. In the present study, a closed-loop management system with a feedback mechanism has been built to ensure continuous optimization for DCT Sub-biobank.

Methods: A biosamples collection program was designed according to feedback in work experience and preventive actions were developed for possible challenges. Each ultra-low temperature freezer was equipped with an alarm system responsive abnormal temperature. Manual monitor of the temperature was performed to avoid errors in the alarm system. The freezers and centrifuges were calibrated at least once a year. Each step, procession and storage, was strictly implemented with standard operating procedures (SOPs) and addressed in detail. Blood was centrifuged immediately, and different components, like serum or plasma, were aliquoted and stored. The quality control information of samples was required to be returned to us. Based on the feedback, we conducted training courses and developed SOPs to achieve continuous improvement.

Results: In the past two years, DCT Sub-biobank has supported 19 projects of drug clinical trial, which were about drug development studies from phase I to Phase III. A total of 136 SOPs in managing and operating have been developed. Up to September 2019, there were 234 cases, 1,465 vials samples in DCT Sub-biobank. The collected samples covered various kinds of tumor. As for the specimens on research distribution, they included 1,144 serum, 27 plasma, and 14 blood cells. Among distributed samples the concentration and purity parameters were qualified according to the feedback of sample users.

Conclusion: Experience with sample collection and storage of the DCTs demonstrated that the closed-loop management system is a quality assured and proven method. However, the development of our DCT Sub-biobank is still at an early stage. There are many challenges for DCT Sub-biobank to be a mature biobank system.

\section{PL-12 Planning for the Unexpected: Creation of a "Mirror Site" as a Part of Business Continuity and Emergency Planning in a Biorepository}

\section{S. Lamers}

\section{Hamilton Health Sciences, Clinical Research Laboratory} and Biobank, Hamilton, Ontario, Canada

The Clinical Research Laboratory and Biobank (CRLB) is an ISO15189Plus-accredited facility within Hamilton Health Sciences, offering processing, storage, and analytical capabilities for biological specimens collected as part of research studies and clinical trials. Our mission is to promote research, innovation, and exemplary professional standards in the research community.

As the collection surpasses its current four million specimens in long-term storage, our facility is realizing the risks and challenges that biorepositories face to ensure the security and integrity of the biospecimens is maintained. Such challenges include how the facility can be monitored continuously, is there contingency planning for equipment failures and infrastructure damage, and implementing risk assessments.
The CRLB has implemented an extensive business continuity plan for managing such challenges and implementing preventative measures for day-to-day risk mitigation. However, as many of the biospecimens have been collected over the past 15-20 years from all regions of the globe, and have also been clinically characterized, their research potential is invaluable and irreplaceable. Therefore, in conjunction with the Population Health Research Institute, the CRLB is developing plans for a future "Mirror Biobank Site" that will permit storage of specimens from each participant at two sites to create redundancy and increase specimen security.

The importance of establishing contingency planning for biorepositories will be emphasized, with day-to-day practices to minimize risk being discussed. Furthermore, the action plan for implementing the Mirror Biobank Site for the CRLB will be presented, including details on the appropriate infrastructure requirements for storage capacity, storage medium, and support for staff and day-to-day operations.

\section{PL-13 What do Researchers Want from Biobanks? Results of an Online Survey}

A. Rush ${ }^{1}$, D. R. Catchpoole ${ }^{2}$, R. Ling ${ }^{3}$, A. Searles ${ }^{3}$, P. Watson ${ }^{4}$, J. Byrne ${ }^{5}$

${ }^{1}$ The Children's Hospital at Westmead Clinical School, University of Sydney, Sydney, New South Wales, Australia, ${ }^{2}$ Children's Cancer Research Unit, The Children's Hospital at Westmead, New South Wales, Australia, ${ }^{3}$ Health Economics, Hunter Medical Research Institute, Newcastle, New South Wales, Australia, ${ }^{4}$ BC Cancer, Vancouver, British Columbia, Canada, ${ }^{5}$ NSW Health Statewide Biobank, Sydney, New South Wales, Australia

Background: The purpose of biobanking is to provide biospecimens and associated data to researchers, yet the perspectives of biobank users have been under-investigated. This study aimed to ascertain the opinions of researchers concerning biobanking services.

Methods: A survey was developed that requested information about researcher demographics, localities of biobanks accessed, methods of sourcing biospecimens, and opinions on topics including application processes, data availability, access fees, progress-reporting requirements, and return of research results. There were 23 multiple choice/check box questions, four questions with a 5-point Likert scale, and one free text question. A web link for the survey was emailed via two New South Wales research network mailing lists.

Results: Responders worked at universities $(n=22 / 43$, $51 \%)$, medical research institutes $(n=18 / 43,42 \%)$, and/or hospitals $(n=15 / 43,35 \%)$, and $n=16 / 43(37 \%)$ were basic laboratory scientists, $\mathrm{n}=16 / 43(37 \%)$ translational researchers, $n=4 / 43(9.5 \%)$ clinical researchers, and $n=7 / 43(16.5 \%)$ other researchers. Most respondents sourced research biospecimens from an existing biobank $(n=19 / 28 ; 68 \%)$ and/or created their own collections $(n=14 / 28 ; 50 \%)$. Respondents were mostly satisfied (defined as scoring $>3$ on the Likert scale) with biobank application processes $(24 / 27,89 \%)$, the formats, types, and numbers of biospecimens available $(22 / 27,81 \%)$, and the format and amount of biospecimen-associated data available $(18 / 28,64 \%)$, such that the majority agreed that biospecimens and data were fit for purpose $(22 / 26,85 \%)$. The most frequently reported influencer to utilise a particular biobank was 'proximity' ( $\mathrm{n}=15 / 28 ; 54 \%)$, followed equally by 'cost' and 'recommendation or reputation' $(n=13 / 28 ; 46 \%)$. As areas for improvement, respondents reported the need for more clinical 
data $(n=21 / 28 ; 75 \%)$, higher quality annotated data $(n=15 / 27$; $56 \%)$, and more data in a suitable format $(n=14 / 27 ; 52 \%)$.

Conclusion: While biobank research users are generally satisfied by biobank services, the clinical annotation of biospecimens could be improved, suggesting the need for closer collaborations between biobanks and clinical data services.

\section{PL-14 Continuous Professional Development in Qatar Biobank}

\section{Hannigan}

Qatar Biobank, Qatar Foundation, Doha, Qatar

Background: Training and staff development can have a positive impact on organisational performance and growth Dedication and commitment to continuous professional development can result in improved organisational performance, increased employee morale, and motivation amongst staff as well as reducing accidents and errors in the workplace. Qatar Biobank (QBB) places great emphasis on the importance of human potential by ensuring the development of all staff to ensure they have the knowledge and skills required to enable them to contribute to sustaining a prosperous organisation.

Methods: A continuous development programme for the staff of QBB was created following a needs assessment of the organisation and of each department. Training requirements were identified through many sources including 1-1 interviews with department managers and review of the strategic business objective requirements to ensure that key performance indicator targets were being reached. Protected training time was negotiated with senior management to allow staff whose roles are operations dependent to be released from duties to attend training.

Results: In the nine months from January, 2019 until the September 30, 2019, 99 training sessions were available to staff; 11 core mandatory topics were identified and conducted, to ensure that staff had knowledge of QBB common procedures such as Information Security and Risk Awareness. Sixty-two department-specific trainings were arranged which included new equipment training and awareness sessions. The remaining 37 trainings were job-specific requirements including induction and orientation, newly promoted staff, or staff who have had a deficiency identified through the internal appraisal process.

Conclusion: Ongoing training and continuous development ensure that staff are well prepared with the knowledge and skills to complete their jobs correctly and safely while improving staff motivation and maintaining staff retention. Qatar Biobank staff are true assets for the organisation, feeling valued through the development of their knowledge and skills to prepare them for their roles. A well-educated and developed workforce will contribute to the quality of the data and biological samples collected and provided to researchers, which will ultimately affect the quality of the outcome of the research.

\section{PL-15 Development of an Integrated Biobank Data Management Software System for Use by Biobank Staff, as well as for Researchers to Access Required Samples}

\author{
S. Jahangiri ${ }^{1}$, R. Vari $^{2}$, C. Librach ${ }^{3,4}$ \\ ${ }^{1}$ Biobank, CReATe Fertility Centre, Toronto, Ontario, Canada, \\ ${ }^{2}$ IT, CReATe Fertility Centre, Toronto, Ontario, Canada, \\ ${ }^{3}$ CReATe Fertility Centre, Toronto, Ontario, Canada, \\ ${ }^{4}$ University of Toronto, Toronto, Ontario, Canada
}

Background: The CReATe Biobank, the first and the only certified Canadian reproductive biological materials biobank, banks a variety of sample types associated with de-identified clinical and demographic data from patients undergoing fertility treatments. The biobank was originally established in 2015 to meet internal research demands, but has grown to support an increasing number of national and international collaborations. To address changing needs and to provide multi-disciplinary data integration, we developed a userfriendly database that enables us to efficiently load, query, and document data that comply with national privacy legislation (PHIPA) and international research ethics (TCPS2) as well as biobanking guidelines (ISBER).

Methods: This database is a browser-based application with a robust SQL back-end. This database allows for multi-device usability with the security, expandability, and stability that Microsoft SQL offers. The premise of the design was to improve the accuracy and efficiency, without adding additional processes at the user level. This on-premises solution was designed to be run on a closed network in order to comply with even the most stringent security requirements.

Results: It currently manages more than 10,000 samples and contains information about biospecimens, patients, data, and everything related to repository management (users and access restrictions) in which all personal identifiers are encoded in an anonymized fashion. The web portal has two graphical interfaces for two groups of authorized users, the biobank staff and researchers, to manage and access data, respectively. The researcher portal is designed for internal researchers only, to view de-identified samples and data and make their requests.

Conclusions: Numerous software solutions are developed for integrated biobanking management. However, none of them supply a flexible model for fertility biobank customization. Therefore, we developed an integrated informatics platform where data are fully traceable and can be securely and efficiently stored, retrieved, and analyzed without dealing with the technicalities of the data grid.

\section{PL-16 Root Cause Analysis (RCA) of Pre-Analytic Workflow: A Case Study of Specimens Placed on Formalin}

L. T. Wiehagen ${ }^{1}$, C. Kline ${ }^{1}$, R. Dhir ${ }^{2}$

${ }^{1}$ School of Health Sciences, University of Pittsburgh, Pittsburgh, Pennsylvania, United States, ${ }^{2}$ Department of Pathology, University of Pittsburgh, Pittsburgh, Pennsylvania, United States

Background: The Pitt Biospecimen Core (PBC) at the University of Pittsburgh is a large, multi-site academic biorepository in Pittsburgh, PA. The PBC has a quality reporting system where all deviations from established protocols are tracked and monitored through the Quality Assurance Committee. Through our Deviation Management System a trend was discovered where valuable, consented samples were not being procured because they were placed on formalin prior to collection, rendering them unsuitable for researcher needs. The deviation reports for these events were compiled and grouped based on hospital site. There were 15 events identified. Further breakdown by site showed that this was primarily an issue at one facility, UPMC Magee, where there were 1.16 events per month.

Methods: The first step was to understand the process. Three process maps were created: 1) PBC Notification Workflow: the information pathway alerting the $\mathrm{PBC}$ of a potential consented 
collection, 2) PBC Procurement List workflow: the PBC process alerting pathology staff that a patient is targeted for collection, and 3) Specimen Flow from Procedure to PBC Collection: selfexplanatory.

These process maps and the 15 deviation reports were used to compile an Ishikawa Diagram of the potential issues a specimen may encounter. All instances of specimens placed on formalin were categorized. The most prevalent cause was identified as "PA not checking the procurement list."

Once the primary cause of the problem was identified, we performed 5Why analysis. It was determined that the procurement list was not easily accessible to the gross room PAs and thus forgotten. With this information, we developed an intervention.

The following items were implemented: Additional copies of the Tissue Procurement list were placed in the Frozen Section (FS) room, on PBC Bench, at each FS grossing station, and to FS and gross room accessioner. In addition, for breast cases, color-coded labels were provided for PAs to use at their grossing station with patient name and tissue type targeted. A check box was added to the PA Breast worksheet which specifically asks if this case was targeted for research collection.

Results: For 2019, Magee had eight specimens placed on formalin, an average 0.66 specimens per month, with $43 \%$ reduction of specimens placed on formalin.

Conclusion: Root-cause analysis can aid in reducing human errors related to systemic causes.

\section{PL-17 Biobanking in a Competitive Environment: Qualifiers and Winners}

\section{E. Steinfelder}

\section{Thermo Fisher Scientific, Nijmegen, Netherlands}

Why do researchers choose specific biobanks like UK Biobank, IBBL, or Biobank Graz as valuable resource for their new research related to personalized medicine? Why are representatives of the Netherlands Cancer Institute, Estonian Genome Center, and IARC so well represented in the setup of the European Mission on Cancer initiative? Much like companies, biobanks must earn their end-user's loyalty and respect to fruitful partnerships. Terry Hill, former professor at the London Business School, coined the terms 'order winners' and 'order qualifiers' and described what the difference is between the two within a business environment. In many biobanks these two features are not clearly defined and often are mixed up, which results in less optimal performance in sharing samples and data.

Order qualifiers are the competitive advantages that a company, in this case biobank, must demonstrate to possess in order to be a viable competitor in the business arena. If a biobank does not meet these minimum standards, the researchers will ignore or reject the potential partnerships. For biobanks, order qualifiers are quality of the samples or patient consent; researchers expect such features as givens but they do not move the needle when it comes to building new partnerships. Domain expertise, delivery speed, flexibility, reliability, and reputation are examples of order winners and considered competitive advantages why end-users choose specific organizations as partner. The latter are the factors that make the difference, the main reasons why researchers would choose a specific biobank as partner.

Defining exactly which are biobanks order qualifiers and order winners can help them to define their strategy. For a biobank, being certified under the latest ISO standard 20387 can still be an order winner, but will in time become a qualifier, much like solid understanding of GDPR. This presentation will highlight how to get ready for the qualifiers and from there being able to deliver the order winning criteria and further increase the access to and use of samples (and associated data) that are currently stored in biobanks across the globe.

\section{Repository Standards}

\section{PM-01 Best Handling Practices to Ensure Quality Genomic DNA Starting Material for Long-Read NGS Library Preparation}

\author{
K. Luttgeharm, S. Borg
}

Agilent Technologies, Ankeny, Iowa, United States

Superior long-read NGS library preparation and sequencing results start with quality genomic DNA (gDNA). Quality control analysis provides a great deal of information that is required before beginning long-read NGS library preparation including size, smear distribution, and concentration. Quality of the gDNA starting material can be modified by the dynamic environment and handling processes it encounters. In addition, shearing parameters may need to be adjusted based on the size and quality of the gDNA starting material to ensure the right range for library preparation. Sizing of gDNA from different extraction methods and kits was compared on the Femto Pulse system. It was found that sizing of initial gDNA varies greatly depending on the extraction method. The amount of shearing required for different-sized gDNA was also investigated to achieve the optimal gDNA range for library preparation. To determine how common laboratory practices such as pipetting, vortexing, and freeze-thaw cycling impact gDNA quality, commercially available gDNA samples were analyzed for size, smear distribution pattern, concentration, and percent of degradation. It was found that different sample handling practices have varied impact on gDNA sizing, with some, such as highspeed vortexing, having a greater effect on sample integrity compared to gentler handling practices, such as slow pipetting with a wide-bore pipet tip. From this study, we have developed a set of best practices for handling gDNA. Quality control analysis of gDNA and gentle handling practices ensure gDNA integrity and build confidence in the success of downstream applications such as long-read NGS library preparation.

\section{PM-02 Comprehensive Temperature Mapping of Freezers in a Biobank at an Academic Medical Center}

\author{
R. Singh ${ }^{1}$, P. McShane ${ }^{1}$, K. Kesee-Bell ${ }^{1}$, T. Delao ${ }^{1}$, \\ N. Garcia ${ }^{1}$, D. Bernard ${ }^{2}$
}

${ }^{1}$ Houston Methodist Research Institute, Houston, Texas, United States, ${ }^{2}$ Houston Methodist Hospital System, Houston, Texas, United States

Background: The Houston Methodist Research Institute (HMRI) Biorepository stores human tissue specimens for downstream biomedical research. The biorepository's current inventory comprises of more than 150,000 different tissue specimens in 30 ULT (ULT; $-80^{\circ} \mathrm{C}$ ) and three liquid nitrogen freezers across two locations. Regular temperature mapping of these freezers is mandated by accrediting agencies like CAP, as well as by NCI and ISBER. However, these agencies are not very specific as to what number of temperature probes are to be used for mapping a freezer, as well as the location of temperature probes within each freezer during the mapping process. The International Society of Pharmaceutical 
Engineering (ISPE) Good Practices Guide: Cold Chain Management, May 2011, states that nine probes should be used when mapping freezers with a surface area below $2 \mathrm{~m} 3$. The HMRI biorepository plans to temperature map all of its ULT $\left(-80^{\circ} \mathrm{C}\right)$ and liquid nitrogen freezers on a regular basis, by using eight temperature probes per freezer. Freezer volumes are less than $2 \mathrm{~m} 3 /$ freezer.

Methods: A standard operating procedure has been written to temperature map all freezers. Freezers will, henceforth, be mapped on a regular basis. Probe positions and duration of mapping have been clearly defined. Freezers will be mapped with full load, partial load, or empty, as the case may be. Few freezers will be tested for 60-second door-opening protocol, too. All freezers will be mapped for 24 hours and for three hours postdoor opening. Needed equipment, including the data logger and temperature probes, have been rented and purchased, respectively. Necessary forms to document all temperature-mapping details have been designed.

Results: The HMRI Biorepository's temp-mapping exercise launched in November 2019 and is expected to end by March/April 2020. Results available until March 2020 will be collated and presented during the ISBER 2020 conference. It is expected that this temperature-mapping exercise using eight temperature probes will throw some additional insights into mapping of ULT $\left(-80^{\circ} \mathrm{C}\right)$ and liquid nitrogen freezers and may pave the way for standardization of temperature mapping for these freezers across the biobanking community.

Conclusion: A comprehensive temperature-mapping exercise on its freezers is being undertaken by the HMRI Biorepository. Hopefully, this comprehensive procedure will help frame specific temperature-mapping policies for biobanking, which we currently lack.

\section{PM-03 Cold Ischemic Time and Health Status in RNA Quality Control of Cardiac Tissue Samples}

A. F. Williams, K. Tomasek, C. Fulmer, K. Gregory,

E. Brittain, T. Absi, Y. Su

Cardiovascular Medicine, Vanderbilt University Medical Center, Nashville, Tennessee, United States

Background: Ensuring RNA integrity is important in biobanking specimen collection. It is widely accepted that tissue samples used for RNA assays should be flash-frozen within minutes of harvesting. The time from explant to freezing is referred to as the Cold Ischemic Time (CIT). RNA quality is thought to decrease with prolonged CIT; however, no studies have examined this in cardiac tissue specifically. Using both healthy and diseased hearts with various CITs, we were able to examine more closely the factors that affect cardiac tissue RNA quality.

Methods: We analyzed 32 diseased and nine healthy explanted hearts. In reviewing the electronic health record, we collected cross-clamp time, sex, age at transplant, and diagnosis. CIT was calculated by subtracting the cross-clamp time from the time of flash-freezing the tissue specimen. RNA was extracted using the Qiagen RNeasy Mini Kit. RNA quality was measured by RNA integrity number (RIN) values, using the Agilent 2100 Bioanalyzer System at the Vanderbilt Technologies for Advanced Genomics core. A t-test and an ANOVA were used to assess the difference between RIN values of healthy and diseased samples, with the latter adjusting for CIT. Spearman's rank-order correlation was used to examine the relationship between CIT and RIN, adjusting for age, sex, and health status. A p-value $<0.05$ was considered statistically significant.

Results: For the diseased explanted hearts, the mean RIN was $5.49 \pm 0.97$, with CITs ranging from 28-113 minutes. For healthy donor hearts, the mean RIN was $7.01 \pm 1.22$, with CITs ranging from 5-15 minutes. There was initially a significant difference in the mean RIN values between healthy and diseased samples $(\mathrm{p}<0.001)$. However, when adjusted for CIT, there was only a trend toward significance $(\mathrm{p}=0.057)$. In further evaluating whether the observed difference between healthy and diseased RIN values was due to CIT versus health status, no significant correlation was found between CIT and RIN when adjusted for age, sex, and health status $(r=-0.2955, \mathrm{p}=0.120)$.

Conclusion: We found that a CIT up to 113 minutes does not correlate with the RIN of cardiac tissue RNA when adjusted for age, sex, and disease status. Thus, minor delays in freezing time when collecting cardiac samples for biobanking do not seem to affect the RNA quality. However, we did note that cardiac disease status may negatively affect RNA integrity, but further studies are needed to more closely examine this relationship.

\section{PM-04 Qatar Biobank (QBB) Internal Audit (IA) Benefits}

\section{W. M. Lobo, L. Hannigan, S. Fazel}

\section{Qatar Biobank, Qatar Biobank, Doha, Doha, Qatar}

Background: Qatar Biobank (QBB) received ISO 9001:2008 \& ISO 27001:2013 on 4th \& 3rd September 2014, respectively. ISO mandates regular internal audit (IA) as part of performance evaluation.

Methods: QBB has stringently implemented the IA process by:

1) Establishing an independent, cross-functional IA team on the basis of competence, understanding of standards, and certified external trainings.

2) Conducting IA once annually.

3) Reporting findings within five working days from audit date.

4) Reporting corrective actions with risk and resource analysis.

Results: 2014: External expertise were hired to perform IA due to lack of internal competency. Internal staff shadowed these external auditors for hands-on experience to gain understanding of performing audit.

2015 and 2016: QBB performed the entire process of auditing independently. IA was performed on two separate days for 9001:2008 and 27001:2013. Repetition in findings, i.e., common in both standards such as legal, health, and safety sections were found. In the close-out meeting, about $30 \%$ of findings were rejected, due to:

1) Auditee did not understand the auditor's question and hence answered incorrectly.

2) Research participant complaints for not receiving visit or result appointments were taken into consideration in quality KPI.

3) Auditee could not show evidence, due to poor management of documents and records.

In 2016, QBB moved into automation. Labs were automated using in-house LIMS \& major version was updated in the existing clinic information management system (Onyx). Excessive findings were reported in lab and information technology functions.

As the process of IA remained the same, findings were duplicated in closing meetings which were rejected by top management.

2017: QBB recertified itself to 9001:2015 and the concept of integrated management systems encompassing 9001:2015 and 27001:2013 as a whole was introduced. Several common procedures were merged and auditors were further trained to audit both standards at the same time. This led to efficient and timely IA.

2018 and 2019: Yearly external trainings continued and new staff members were recruited to the IA team. New auditors were 
asked to be observers during IA to gain competency as auditors. Though just observers, their reports were taken into consideration for final report.

Conclusions With time, staff member expertise both as auditors and auditee increased. This can be known from the fact that the percentage of findings rejection is now next to nil. In fact, most or all findings that are picked by the external auditors have already been reported in the IA.

\section{PM-05 Proteomics-Based Stability Assessment of Long- term Storage Serum Samples of KBN-affiliated Biobanks}

E. Kim ${ }^{1}$, H. Yoo ${ }^{1}$, S. Lee ${ }^{3}$, S. Shim ${ }^{1}$, H. Nam ${ }^{1}$, S. Lee ${ }^{1}$, B. Ji ${ }^{1}$, M. Lee ${ }^{1}$, K. Wang ${ }^{1}$, J. Lee ${ }^{4}$, M. Kim ${ }^{2}$, J. Jeon ${ }^{1}$

${ }^{1}$ Division of Biobank for Health Science, Center for Genomic Science, Korea National Institute of Health, Korea,

${ }^{2}$ Department of New Biology, DGIST, Daegu, Korea,

${ }^{3}$ Department of Biomedical Science and Technology, Kyung

Hee Medical Science Research Institute, Seoul, Korea,

${ }^{4}$ Department of Applied Chemistry, Kyung Hee University, Yongin, Korea

Korea Biobank Network, consisting of a central National Biobank of Korea (NBK) and 17 regional biobanks, has collected and stored over 10 million vials of serum and plasma samples from 2001 to 2018. Sometimes, old biobanks are asked to provide the stability data of long-term biobanked blood samples to assure reliable analytic results, especially in proteomics and metabolomics. To demonstrate the stability of blood samples with long-term storage, we selected serum and plasma samples $(n=476)$ from the National Biobank of Korea and generated proteomics data in various time points of storage $(<1,1,5,10$ years $)$ and different storage temperatures $(-80$, $-150^{\circ} \mathrm{C}$ ). Proteomics data with representative 40 peptides were produced and analyzed by multiple reaction monitoring-mass spectrometric method. As observed in principle components analysis, peptide profiles of test samples stored for over 10 years were comparable with that of test samples stored for less than one year where the samples were stored in $-150^{\circ} \mathrm{C}$. In addition, the similar peptide profiles were observed in test samples stored at $-150^{\circ} \mathrm{C}$ and $-80^{\circ} \mathrm{C}$. However, a peptide profile was strikingly changed where the blood samples were stored at $-20^{\circ} \mathrm{C}$ for over 10 years. Taken together, this work suggests that serum samples are highly recommended to be stored at $-80^{\circ} \mathrm{C}$ or below to obtain reliable proteomics data from long-term biobanked samples.

\section{PM-06 Service Delivery through ISO9001 Quality Management System: A National Health Laboratory Service Biobank Perspective}

\section{Maseme}

Biobank, National Health Laboratory Service, Roodepoort, Gauteng, South Africa

Background and introduction: A quality management system (QMS) is important in any organization as a means of ensuring quality of the products and service(s) rendered. Since the inception of the National Health Laboratory Service (NHLS) Biobank, the overarching objective for a sound and effective QMS has been to ensure service delivery compliance with international standards, particularly ISO 9001, and maintenance of certification henceforth. The NHLS Biobank was certified for ISO 9001:2015 in April 2019 and this abstract provides an overview of its certification process.
Methods: The NHLS Biobank conducted a gap analysis and internal audit in preparation of ISO 9001:2005 certification process. An external audit was conducted subsequent to this in two stages by the SGS certification company. The objective of stage 1 was to determine if there was QMS in place, a prerequisite for stage 2, which was to determine conformity of the Biobank to the QMS.

Results: Five minor non-conformities (NCs) were identified from clauses 4 (business environment and scope) and 8 (operational processes) of the ISO 9001 checklist during gap analysis. The internal audit resulted in 11 identified NCs (four major and seven minor) from clauses 4, 8 and 7 (documented information). The Biobank successfully met the objectives of stage 1 audit with recommendations raised in preparation for stage 2. Stage 2 audit resulted in four NCs (one major and three minor) which were reviewed by the external auditor after being cleared internally, following which the Biobank was recommended for certification.

Conclusion: The gap analysis and internal audit proved to be a success in preparation for ISO 9001 certification. The NHLS Biobank certification is a successful milestone as the goal for certification was achieved before the set target for implementation of ISO 9001 certification by the year 2020 reflected in the strategic plan. The NHLS Biobank will maintain the ISO 9001 certification through maintenance of QMS and this will be assessed through annual internal and external audits.

\section{PM-07 Freezing Procedures of RNA PaxGene ${ }^{\circledR}$ Tubes and their Influence on RNA Quantity and Quality}

T. Heimberger, S. Sandke, T. Weis

Heidelberg CardioBiobank (HCB), University Hospital Heidelberg, Heidelberg, Germany

Background: The Heidelberg CardioBiobank (HCB) operates as a hospital-integrated biobank as well as a core biobank for several national and international clinical trials and research projects. In the daily routine of a hospital, blood collection for clinical studies is sometimes subordinated to patients' treatment and care. Despite stringent standard operating procedures, the time of the biomaterial transfer to the biobank and therefore also the processing of blood samples can vary. With this analysis we investigated the influence of different incubation times and storage procedures of RNA PaxGene ${ }^{\circledR}$ tubes and their impact on RNA quantity and quality.

Methods: For investigating the influence of different incubation times, RNA PaxGene ${ }^{\circledR}$ tubes (BD Biosciences, Franklin Lakes, NJ) have been placed at room temperature (RT) for 2, 4, and 6 hours (h) before direct storage at $-80^{\circ} \mathrm{C}$. To analyze the impact of the freezing method, we also performed an "indirect" storage procedure where RNA PaxGene ${ }^{\circledR}$ tubes were also incubated for 2,4 , and $6 \mathrm{~h}$ at RT and then stored for $24 \mathrm{~h}$ at $-20^{\circ} \mathrm{C}$ before their final storage at $-80^{\circ} \mathrm{C}$. RNA concentrations were determined with the Quantus ${ }^{\mathrm{TM}}$ Fluorometer (Promega, Madison, WI). As a quality marker, the RNA integrity number (RIN) was determined by Bioanalyzer Agilent RNA 6000 Nano Kit (Agilent, Santa Clara, CA).

Results: The measurement of the RNA amounts revealed that the incubation of PaxGene ${ }^{\circledR}$ tubes for $2 \mathrm{~h}$ at RT followed by direct storage at $-80^{\circ} \mathrm{C}$ leads to the highest RNA yields. However, the mean values of the RINs are without any major differences in these tested RNAs. In contrast, in the analyses of the indirect storage procedure, we did not observe any significant differences in the amount of RNA. But here the RIN values indicate that incubation for $6 \mathrm{~h}$ at RT, before indirect storage, leads to a lower RIN value. 
Conclusions: In the era of precision medicine the requirements for a hospital-integrated biobank are multifactorial: operating as a classical biobank for the general storage of biomaterial, supporting clinical studies with collecting and processing of biomaterial, and also providing high-quality biomaterial and data for research. Therefore, we continuously evaluate and adapt our workflow processes to warrant the highest quality of biomaterial. By analyzing the freezing procedures for RNA PaxGene ${ }^{\circledR}$ tubes, we discovered that the incubation time and storage process influences the quantity and quality of RNA.

\section{PM-08 Quality Management System: A Key Performance Indicator of BioBank Operations}

S. Paul, H. Wagner, N. Fleshner

Surgical Oncology, University Health Network, Toronto, Ontario, Canada

Banking of high-quality appropriately consented human biospecimens is crucial for the understanding of disease pathogenesis and translation of such knowledge into improvements in patient care. The McCain GU Biobank Quality Management System (QMS) ensures that a biospecimen meets accepted quality standards which will aid in minimizing biospecimen variability that could affect the interpretation of scientific results. The QMS encompasses standard operating procedures (SOPs) and best practices, equipment calibration and maintenance, specimen inventory, and clinical data quality assurance (QA) as well as client satisfaction survey on overall BioBank service.

The McCain GU Biobank has established SOPs for carrying out QA and quality control for Biobank effectiveness. Specimens and data are assessed on a monthly basis to ensure accuracy and quality. A client satisfaction survey has also been employed to receive feedback on sample request, distribution process, and sample quality. As part of the Biobank specimen inventory, QA liquid biospecimens (blood and urine) are physically evaluated based on several parameters, including the location of a specimen within the cryostorage inventory, available volume, condition of storage vials, as well as the accuracy of the label. QA results are then compared with specimen inventory database for accuracy and used as key performance indicators for the overall effectiveness of the quality management program.

We have performed quality assurance on different sample types banked under various collection protocols. The specimens are assessed for correct storage locations in cryostorage inventory, sample availability, available volume, and labeling accuracy. Of the samples assessed, $100 \%$ are found to be in their correct storage locations with $100 \%$ labeling accuracy. Specimens are also checked for volume accuracy and it has been found to be over $90 \%$. Incident reporting system has been employed to track and report deviations so that CAPA can be implemented when necessary. Annual freezer and equipment calibration ensure specimens are processed and stored as per SOP and industry best practices.

The quality and integrity of biospecimens are critical components of BioBanking operations. For biomarker discovery, it is essential that experimental results are consistent and reproducible. Effective and efficient QMS in Biobanks can promote reliability on biospecimens and clinical data which are key contributors to cutting edge translational research.

\section{PM-09 Evaluation of DNA Integrity Number as a Quality Control Tool for Genomic DNA}

A. B. Hughes ${ }^{1}$, C. Bender ${ }^{1}$, C. Lwin ${ }^{1}$, A. Naik ${ }^{1}$, M. Reeves ${ }^{1}$, K. Goetz ${ }^{1}$, B. Guan ${ }^{1}$, R. Hufnagel ${ }^{1}$, S. J. Tumminia ${ }^{2}$
${ }^{1}$ OGVFB, National Eye Institute, Bethesda, Maryland, United States, ${ }^{2} \mathrm{OD}$, National Eye Institute, Bethesda, Maryland, United States

Background: The eyeGENE ${ }^{\circledR}$ Biorepository was established in 2006 at the National Eye Institute/National Institutes of Health to serve as a resource for vision researchers. Genomic DNA extracted from whole blood samples was banked for research use and clinical genetic testing. eyeGENE ${ }^{\circledR}$ has served as a model of best practices by iteratively incorporating quality assurance and control (QA/QC) procedures. In addition to spectrophotometry, automated electrophoresis can be used as a complementary QC tool to assess DNA integrity and fragmentation. Recently, we reviewed DNA integrity number (DIN) and DNA quality. DIN reliability and correlation with spectrophotometry measurements tested whether DIN is a dependable measure of different aspects of DNA quality.

Methods: To evaluate correlation with spectrophotometry, 404 DNA samples were tested on Agilent TapeStation automated electrophoresis and DeNovix spectrophotometer. Duplicate runs on two high-quality samples were performed to assess DIN consistency across intra-sample replicates within and outside of the functional DIN range $(5-300 \mathrm{ng} / \mu \mathrm{L})$. Each sample was diluted to $400 \mathrm{ng} / \mu \mathrm{L}$ ( 24 replicates per sample) and $200 \mathrm{ng} / \mu \mathrm{L}$ ( 24 replicates per sample). To test DIN as a reliable measure of DNA quality and fragment size, four DNA samples were sonicated at six different durations to target different fragment sizes (24 samples).

Results: DIN showed tight correlation to $260 / 280$ optical density but not 260/230. For the 346 samples with 260/ $280>1.80$, the DIN range was 5.9-9.9. For testing DIN reliability within and outside the DIN functional range (5-300ng/ $\mu \mathrm{L})$, the maximum standard deviation for the intra-sample replicates was 0.26 within the range and 0.57 outside of the range. Further, an increase in sonication time had an indirect correlation with DIN and observed DNA fragment size with a Pearson coefficient of $\mathrm{R} 2=0.949$ when fit to an exponential regression.

Conclusions: DIN is a useful DNA quality metric with 260/ 280 optical density. DIN is also reproducible for samples both within and at least $100 \mathrm{ng} / \mu \mathrm{L}$ above the DIN functional range. DIN displayed a strong negative correlation with sonication time, indicating that it is sensitive to differences in fragment size and degradation. We observed no correlation with 260/230 values, suggesting DIN is not sensitive to protein or carbohydrate impurities. While DIN is not a stand-alone QC measurement, it is a valuable and complementary tool for DNA QA/QC in biorepositories

\section{PM-10 Harmonization of Mexican Biobank Network by the adoption of ISBERs Best Biobanking Practices}

A. G. Gámez Medina ${ }^{1,2}$, C. M. Luna Aguirre ${ }^{1,2}$,

M. A. Campomanes Flores ${ }^{2}$, S. Bernal Silva ${ }^{3,4}$,

B. Illades Aguilar ${ }^{5}$, C. R. López Macías ${ }^{6}$, J. P. Reyes Grajeda ${ }^{7}$, A. Ulloa Aguirre ${ }^{8}$, G. Vizcaíno Tapia ${ }^{8}$, C. Ximénez García ${ }^{9}$, L. A. Herrera Montalvo ${ }^{10}$, L. M. Ruiz-Godoy-Rivera ${ }^{10}$, R. López Ridaura ${ }^{11}$, M. Lajous Loaeza ${ }^{12}$, H. A. Barrera-Saldaña ${ }^{1,13}$

${ }^{1}$ Biobank, Vitaxentrum, Monterrey, Nuevo León, Mexico, ${ }^{2}$ Biobank, Innovita, Mexico City, Mexico, ${ }^{3}$ Microbiology Department, Autonomous University of San Luis Potosí, San Luis Potosí, Mexico, ${ }^{4}$ Research Center for Health Sciences and Biomedicine, Autonomous University of San Luis Potosí, San Luis Potosí, Mexico, ${ }^{5}$ School of Chemical-Biological Sciences, 
Guerrero Autonomous University, Chilpancingo, Guerrero, Mexico, ${ }^{6}$ Mexican Social Security Institute, Mexico City, Mexico, ${ }^{7}$ National Institute of Genomic Medicine, Mexico City, Mexico, ${ }^{8}$ National Institute of Medical Sciences and Nutrition Salvador Zubiran, Mexico City, Mexico, ${ }^{9}$ National Autonomous University of Mexico, Mexico City, Mexico, ${ }^{10}$ National Institute of Cancerology, Mexico City, Mexico, ${ }^{11}$ National Center for Preventive Programs and Disease Control, Mexico City, Mexico, ${ }^{12}$ National Institute of Public Health, Mexico City, Mexico, ${ }^{13}$ Ambassador LATAM, ISBER, Monterrey, Mexico

Statement of the Problem: Biobanks are a keystone in the development of early diagnosis, prognosis, and prediction methods for illnesses such as infectious diseases that reduce the life span, quality of life, and productivity of citizens. These health issues are strategic targets for bioindustries, which allows networks like ours to be part of these innovative developments given an implementation of Federated Best Biobanking Practices.

Proposed Solutions: Our purpose is to promote the implementation of ISBER's Best Biobanking Practices (BBP) among biobanks of the "Red Mexicana de Biobancos" (MxBioBan) to leverage our capability to team up with the bioindustry. Innbiogem personnel participated in the translation to Spanish of the Fourth Edition of the BBP of ISBER. Its adoption by the various members of the MxBioBan Network is being diligently promoted, improving the Network to procure biospecimens of value for projects of biomarker discovery for the bioindustry. Properly collecting, processing, and storing every single sample along with its associated data within highquality standards to comply with strict requirements demanded by each protocol is one of the biggest challenges that every Biobank wanting to be productive and sustainable faces.

Conclusions: To guarantee the value of collectively procured biospecimens by the members of a network of biobanks is a prerequisite to adopt homologated operational procedures. ISBER's BBP has been the gold standard for quality control and quality assurance of biospecimens collection in the world.

\section{PM-11 Adapt to ISO 20387 and China Human Genetic Resources Management Regulations by Upgrading Biobank Information System}

Y. Zhang ${ }^{1,2}$, Y. Chen ${ }^{1,2}$, X. Li ${ }^{1,2}$, X. Zhang ${ }^{1}$, T. Huang ${ }^{1}$, P. Zhang ${ }^{1}$

${ }^{1}$ Research Center, Beijing Friendship Hospital, Capital Medical University, Beijing, China, ${ }^{2}$ Beijing Clinical Medicine Institute, Beijing, China

Statement of the Problem: ISO 20387 General Requirements for Biobanking was published in August 2018. One year later in July 2019, China Human Genetic Resources Management Regulations was issued. How to apply these two to our routine work is the most concerned problem.

Proposed Solution: We modified our quality management documents and upgraded and transformed our biobanking information system accordingly. We implemented the requirements of ISO 20387 for the key steps in the system to achieve the traceability of important information. A variety of post roles were preset in the system. Necessary information was collected automatically in the workflow of each post to realize the acquisition of the SPREC. A sample report could generate automatically in the system; quality-related information was reflected in it. To ensure that the source of genetic resources was legal as required, our system had informed consent verification function in the sample-receiving section, which could scan and assign a two-dimensional code to the consent.

Conclusion: Through the upgrading of our system, several problems in the standardized quality system are solved, such as the acquisition of important biological quality information during sample lifecycle, the generation of electronic sample report, the separation of informed consent and sample information, etc.

\section{PM-12 ISO 20387 - A2LA Biobanking Accreditation Program}

C. D. Arant

Life Sciences, A2LA, Frederick, Maryland, United States

The A2LA Biobanking Accreditation Program uses the ISO 20387 standard to promote confidence in biobanking. ISO 20387 contains requirements designed to demonstrate the competence of a biobank's operation and ability to provide biological material and associated data for research and development. Biobank accreditation is a formal recognition by an authoritative third party of the competence of a laboratory to perform specific biobanking activities including acquisition, collection, preparation preservation, testing, analysis, distribution.

Benefits of accreditation to the Biobank:

1) Accreditation by a third party provides credibility to the users of biobanks and establishes a level playing field.

2) The accreditation body's involvement in the conduct of assessments and the review of assessment outcomes creates and maintains consistency between assessments.

3) Accreditation by a third party allows access to experienced, expert assessors who are technically competent in the fields assigned.

4) Use of accreditation bodies that are signatories to the ILAC Mutual Recognition Arrangement provides a high level of confidence in the accreditation body's competence. This confidence is based on the requirement for the accreditation body to undergo routine, rigorous peer evaluations against long-standing international standards for assessing quality as well as on the satisfaction of the laboratories themselves.

Attendees will gain an understaning of the format and requirements of ISO 20387:2018 and how this standard can be used to distinguish and highlight the capabilities of competent biobanking operation.

\section{PM-13 Construction of Standard Operating Procedures for Specimen Collection of High-Altitude Residents}

\section{S. Wang, X. Zhao, Y. Tian \\ Chinese PLA General Hospital, Beijing, China}

High-altitude residents' specimen bank is a significant resource for genomic research and discovery of diagnostic biomarker for high-altitude diseases. However, extreme environment and climate on plateau areas always creates difficulties for the specimen collection. Researchers or staff from plain areas may have altitude stress which would impact collection work development. Furthermore, considering the lag of experiment condition, specimens cannot be well prepared and processed before and after collection. Another handicap is difficult transportation, which cannot ensure the specimens' quality while 
transported form one site to the destination. Therefore, standard operating procedures (SOPs) of specimen collection, processing, transportation, and storage are of great significance. We have developed a high-altitude residents biobank with more than 300 cases covering thousands of varied specimens, such as serum, plasma, leukocytes, urine, stool, and saliva. All the SOPs are set up and applied during the specimen collection.

In order to cover the whole process of biobank operational mechanism, we lay down the procedures containing the precollection preparation notes, such as donator healthcare and psychological care and special collector for low-atmospheric pressure avoiding poor blood drawing volume. Collection procedures of blood, urine, stool, and saliva would be applied according to the same standard respectively, which is imparted to the nurse and donator by the researcher before collection. All the specimens would be processed and split into aliquot and filled into tubes, which are stored in $-80^{\circ} \mathrm{C}$ ultra-low temperature refrigerator. We also formulate an appropriate transportation procedure to make sure the specimen's stability and repeatability of measurement. Specimens transported from plateau area to plain destination are controlled under $-80^{\circ} \mathrm{C}$ by dry ice. We then develop a professional information collection and processing software for the information storage of donators' specimen and phenotype. At last, we formulate a wellestablished management system to make sure that the specimen information is correct and intact, and moreover the specimens are collected with normalization and legality. Only by conforming to the SOPs, specimens collected at different times can reflect the overall condition of donator's physical state and the research data can be as real as possible.

\section{PM-14 International Biobanking Standards and Best Practices for Assuring Quality Repository Management}

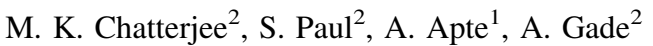 \\ ${ }^{1}$ CloudLIMS.com, Wilmington, Delaware, United States, \\ ${ }^{2}$ CloudLIMS Lab Solutions (India) Pvt. Ltd, Indore, MP, India
}

Statement of the Problem: Effective quality biobanking is underpinned by standardization and best practices. Biobanks should follow international standards and best practices to ensure the biospecimens they store are fit for research purposes and the results of studies conducted using biospecimens are reproducible. One new international standard that promotes confidence in biobanking and plays a crucial role in the standardization of biobanking practices is ISO 20387: 2018. It specifies the technical and quality requirements for the competence, impartiality, and consistent operations of biobanks.

For quality biobanking management, ISBER Best Practices (BP) serve as one of the key documents to supplement the ISO 20387:2018 standard, harmonizing scientific, technical, legal, and ethical issues. ISBER BP enable biobanks to develop standardized methods for collection, handling, storage, retrieval, and distribution of specimens, enabling them to provide high-quality specimens that meet necessary regulatory and statutory requirements.

Since biobanking operations vary widely, it is a challenge to implement the standards and BP. However, the following are the common areas between the two:

1) Tracking samples and managing patient consent.

2) Training of staff, regular monitoring of their performance, and documentation of training records.
3) Maintaining an audit trail with read-only access to automatically record data changes with a date and time stamp.

4) Managing documents, such as material transfer agreements and standard operating procedures.

5) Labeling of specimens with a unique ID not derived from the personal information of donors.

6) Conducting internal audits.

Proposed Solution: To keep track of the recommendations under the standards and BP, a need was felt to automate the processes of securely managing specimen data, patient consents and anonymization of ePHI, maintaining an audit trail, training of staff, etc. Furthermore, assigning role-based access of confidential data to authorized personnel and managing documents are needed to establish security. For supporting the automation requirements, a laboratory information management system (LIMS) was identified as a tool for the quality assurance of biospecimens.

Conclusion: Biobanks should meet the requirements of ISO 20387 and follow best practices for the quality management of biospecimens. Though the processes can be met manually, a LIMS offers an automated solution to meet the various requirements of ISO 20387 and ISBER BP, assuring quality biobanking.

\section{PM-15 An Investigation into Total Quality Management and its Impact on Organisational Performance in the Biobank of the National Health Laboratory Service (NHLS)}

\section{B. Duma}

\section{Biobank, National Health Laboratory Service, Centurion, Gauteng, South Africa}

Background: The National Health Laboratory Service (NHLS) is the part of the South African Department of Health. NHLS has different divisions including the Biobank. The Biobank is responsible for collecting and storing biomaterials and associated data for short-term and long-term use by researchers. Implementation of total quality management (TQM) at the biobank will help to improve processes and the way the biobank functions in terms of storage of biomaterial and data. The organisational performance will be assessed and monitored throughout. The biobank staff and processes will be affected. Implementation of TQM presents the opportunity to improve on biobanking service delivery, manage of biomaterial and safety of associated data, and improve organisational performance. This research study was based on qualitative assessment of the impact of the implementation of TQM using ISO 9001 principles on organisational effectiveness.

Research: Involved planning and design of the study. The researcher was the data collection instrument and the samples were the participants who met the eligibility criteria. A nonprobability sampling design was used. The data collection used unstructured interview with each participant. Thematic analysis was used to code the data and identify emerging themes of the study. The study was complemented with an indepth interview of each participant to focus on the quality management experiences.

Conclusion: Implementation of the quality management system (QMS) in the biobank has been carried out effectively with the available resources. Successful implementation used appropriate biobank methods, staff engagement, and training. Using quality training and meetings appear to be effective with management involvement in all the implementation processes. 
The NHLS for this study concluded that the implementation of a QMS in line with the ISO 9001 adds significant value and has positive impact on an organisation and its customers. It increased employee and customer satisfaction. TQM use requires continuous process improvement, process approach techniques, and a measurement-based system for performance that requires an advanced degree of system maturity that is mostly related to creating a culture of process improvement. The availability of high-quality biomaterial for research purposes requires the development of standardised methods for collection, handling, storage, retrieval, and distribution. The implementing of the TQM in the Biobank showed improvement and positive organisational performance.

\section{PM-16 NMR-Based Quality Control and Generation of Standardized Spectral Information}

\section{Cannet, E. Nascimento}

\section{Bruker BioSpin, Rheinstetten, Germany}

Statement of the Problem: Biobanks are increasingly large and diverse. Standardization is in discussion and biofluids stored are not always well characterized.

Proposed Solution: NMR is one of the two analytical methods most important for mixture analysis. Based on its unmatched reproducibility, transferability, and robustness, it is ideally suited for large-scale metabolomics screening. As such it is also an important tool for biobanking. A dedicated platform under complete standardization has been developed, allowing to combine NMR spectral data produced worldwide for population based studies, for longitudinal studies on individuals and for clinical trials.

The NMR platform and its tools are especially suited for biobank quality control (QC), as it can deliver a comprehensive quality status report of aliquots to be stored in a biobank. It includes information on matrix identity and integrity; contamination and impurities; indicative parameters, e.g., fasting state; NMR preparation and measurement. During the QC process a NMR spectrum is generated under completely standardized conditions, which can be stored in the biobank as well. Spectra can be analyzed automatically, generating information on small molecule quantification and lipoprotein main and subclass analysis in plasma/serum or endogenous, drug metabolite, and disease markers quantification in urine and classification results against normality models. NMR also offers new concepts for support of clinical trials through NMR spectra. Due to the reproducibility and standardization in NMR, spectra of controls and defined disease status can be pulled together on a worldwide bases.

Conclusions: Thus, instead of new aliquots, NMR spectra can be used to deliver, for example, control spectra from which normal models can be built or models for selected diseases. This leads to reduction of time and effort for clinical trials. Examples for QC and analysis tools will be given.

\section{PM-17 A Biobank Derived from Systems Toxicology Research and Assessment Laboratories}

\section{S. Ansari, E. Dargaud}

Research Technologies, Philip Morris International - Science \& Innovation, Neuchatel, Switzerland

Statement of the Problem: Philip Morris International is a pioneer in systems toxicology research and assessment and has invested intensively in its evolution. The systems toxicology team has performed numerous studies with a broad range of samples, including material from in vitro, preclinical in vivo, and clinical studies.

With a significant growth over time in our research capabilities as well as the number of samples used for omics endpoints, we had to put in place sample management processes and appropriate sample storage facilities. However, with our strong dependence on omics endpoints and systems toxicology data analysis, detailed sample information and sample event tracking became very critical. In fact, in some studies, effects within the data were explained through confounding effects resulting from unplanned but tracked events in the sample lifecycle. These were, in many cases, identified and computationally discriminated from the experimental factors, ultimately allowing us to better design our future experiments.

Proposed Solution: To address the sample management challenge, we looked into sophisticated data management solutions. We therefore implemented a computerized system-a Biobank Information Management System (BIMS) - to track sample events and metadata. Integrating this system with related systems such as the laboratory information management systems helped improve the sample management process and make the omics endpoints robust enough to qualify as assessment endpoints for regulatory submissions. Consequently, we also applied the ISO9001 corporate quality management system (QMS) to these processes, systems, and endpoints and adopted system and method validation, change control, procedure management, training management, data archiving, and other processes. The direct impact was a drop in operational throughput and flexibility. However, in the course of this migration, we adopted QMS processes and new methods based on agile implementation approaches and riskbased assessment, which allowed quicker responses to changes while maintaining operational throughput and scalability.

Conclusions: In this work, we will outline the steps and challenges of this migration process and highlight critical success factors and lessons learned.

\section{Underserved and Vulnerable Communities}

\section{PN-01 Establishing the First Rare Disease Biobank in South Africa: Goals and Challenges}

\section{E. H. Conradie, B. C. Vorster, M. Dercksen}

Centre for Human Metabolomics, North-West University, Potchefstroom, North-West Province, South Africa

Statement of the problem: The North-West University's Centre for Human Metabolomics (CHM) is currently in the process of establishing the first rare disease biobank in South Africa (SA) and Africa. The CHM Biobank's main focus is on the collection of samples and information for rare congenital anomalies. The need for such a biobank was identified at the CHM's diagnostic lab. Feedback towards this initiative was overwhelmingly positive at the first stakeholder meeting in August 2019. However, gaining support from the public sector and recruiting of participants have proven to be challenging. Problems experienced to date include: lack of support from government and clinicians, lack of knowledge on rare disease (patients and clinicians), public health care focus not directed towards rare diseases, patients not returning for follow-up visits, and unwillingness to participate due to fear of exploitation.

Proposed solution: The CHM Biobank's vision and goals are aligned to address a national and international research need; it will provide a valuable resource for scientists to improve what is known about these diseases, to better understand how these 
diseases work, to optimise diagnostic methods, and to possibly develop treatments. The genetic variability of the SA population provides added value to the rare disease biobank. The following actions are proposed towards a solution for the problems experienced: intensified literature review to highlight the possible benefits of this initiative to SA, engagement with stakeholders to obtain written statements of support, revision of recruitment protocol in oversight of ethics committee, community engagement to address the fear of being exploited, conducting surveys amongst healthcare professionals and patient support groups to investigate the recruitment barriers, and developing workshops and training material to address the lack of knowledge on rare diseases.

Conclusions: The CHM Biobank addresses a global scientific need and is widely supported by stakeholders both local and international. Through the setup process, it came to light that there is a lack of knowledge and importance of rare disease research in South Africa. This has led to problems experienced in gaining support from the public sector and in recruiting participants. Revision of protocols is ongoing, showing some success to date, but requires further optimisation and input from the global biobanking and research community.

\section{PN-02 Specimen Collections in Marginalized Populations: Experience from HCV Trials}

\author{
S. Ongarello ${ }^{1}$, E. Ivanova ${ }^{2}$ \\ ${ }^{1}$ Data Services and Biobanking, FIND, Geneva, GE, \\ Switzerland, ${ }^{2}$ HCV Programme, FIND, Geneva, Switzerland
}

Statement of the Problem: More than 71 million people worldwide are hepatitis $\mathrm{C}$ virus (HCV)-positive and fewer than $20 \%$ of them are aware of their status. HCV prevalence is high among marginalized populations, such as people who inject drugs (PWID) and men who have sex with men. Reaching these populations and bringing them to the $\mathrm{HCV}$ care cascade is crucial to ensure control and management of the disease, and to monitor disease prevalence. Recruitment and collections are complex: individuals at risk face enormous challenges in seeking care as drug use is punishable in some countries, test costs are high, and access to health centers is difficult. HCV samples used by test manufacturers usually come from blood donations, mostly from developed countries (Europe \& U.S.), and are not representative of $\mathrm{HCV}$-infected populations; $>50 \%$ of $\mathrm{HCV}$-infected samples belong to high-risk populations, such as PWID.

Proposed solution: FIND seeks to reach marginalized populations through clinical trials in partnership with in-country organizations routinely working with these populations, like harm-reduction sites (HRS) and community-based organizations (CBO). Leftover blood samples (plasma, serum, venous whole blood, etc.) are stored by FIND in its biobank for use in the future development of HCV diagnostics. Engagement is crucial: FIND offers participants the possibility of being tested free of cost at local HRS. Samples are then sent to central laboratories, and results returned to HRS, thus avoiding the need for individuals to present themselves at main health centers. Follow-up visits are also offered for free.
This model has challenges:

1) Understaffed and inexperienced HRS and $\mathrm{CBO}$ requiring adequate training for clinical trial activities and continuous monitoring

2) Lack of basic testing equipment

3) Difficult logistics for same-day sample transportation in some countries

4) Timely reporting of results

5) Adequate interaction with intended patients

6) Difficulty obtaining samples (haemolysed or viscous blood) can be partially alleviated by offering soft drinks to participants during the consent and interview

Conclusions: The initiation of five studies (in Georgia and Greece) resulted in $>700$ PWID being recruited, tested for $\mathrm{HCV}$, and, when positive, linked to care. Direct access to diagnostics and care at HRS significantly increased enrolment rate. Additional studies in Ukraine and Georgia will start soon. FIND HCV specimen collections will contribute to more reliable development and evaluation of HCV diagnostics.

\section{PN-03 The IARC Biobank and Cohort Building Network (BCNet)}

\author{
Z. Kozlakidis ${ }^{1}$, E. Caboux ${ }^{1}$, S. Villar ${ }^{1}$, M. Henderson ${ }^{2}$ \\ ${ }^{1}$ Laboratory Services and Biobanking, IARC/WHO, Lyon, \\ France, ${ }^{2}$ National Cancer Institute, Center for Global Health, \\ Bethesda, Maryland, United States
}

Statement of the Problem: Biobanks, which facilitate the collection and storage of research-ready high-quality biological specimens and associated data, play a key role in scientific research on disease prevention, screening, and treatment. In contrast to the situation in high-income countries where support might be committed for the longer-term, biobank infrastructures and related guidelines and protocols are much less developed in low-and-middle-income countries (LMICs). This constitutes a serious barrier to high-quality scientific research projects in LMICs.

Proposed solution: In line with IARC's and WHO's mission in contributing to worldwide cancer research, and in collaboration with the U.S. National Cancer Institute - Centre for Global Health (NCI-CGH) and other international partners, such as ISBER and CTRNet, a biobank network (BCNet) was set up as an opportunity for LMIC to work together in a coordinated and effective manner and jointly address the shortfalls in biobanking infrastructure and other shared challenges, including ethical, legal, and social issues. The network will facilitate the sharing of resources and the development of joint projects, strengthening the competitiveness of the LMIC in applying for international funding.

Conclusions: The founding members include 30 representatives from 16 LMICs. Membership is not exclusive and is open to other LMIC biobank institutions that are willing and able to work together for common interest and agree with the principles and practices endorsed by the BCNet governance. 